\title{
10-Step Asymmetric Total Synthesis of (+)-Pepluanol A
}

Po Yuan ${ }^{\ddagger}$ Christa K. G. Gerlinger $\$$, Jan Herberger ${ }^{\ddagger}$ and Tanja Gaich ${ }^{\star}$

Institute of Organic Chemistry, University of Konstanz, Universitätsstrasse 10, Konstanz 78464, Germany

†Institute of Inorganic Chemistry, University of Konstanz, Universitätsstrasse 10, Konstanz 78464, Germany

\section{Table of Contents}

1. General Methods

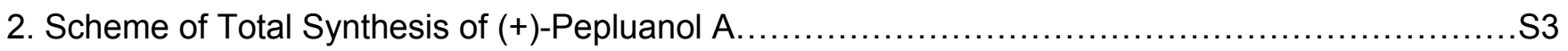

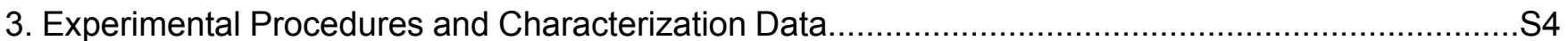

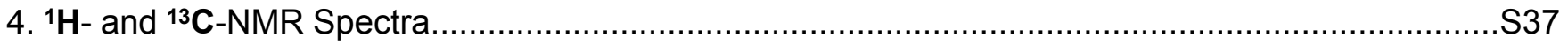

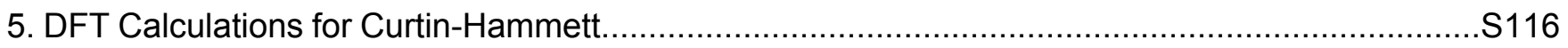

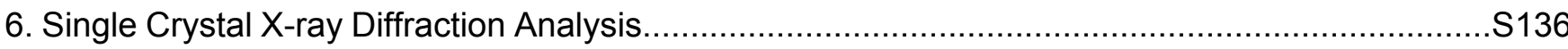

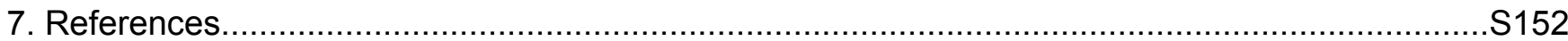




\section{General Methods}

All reactions were carried out in oven- or flame-dried glassware, and all anhydrous reactions were performed under $\mathrm{N}_{2}$. Anhydrous solvents were used for all reactions except solvent mixtures with water unless stated otherwise. All anhydrous solvents (THF, $\mathrm{CH}_{2} \mathrm{Cl}_{2}, \mathrm{Et}{ }_{2} \mathrm{O}$, o-xylene, DMF, MeCN, $\mathrm{MeOH}$, EtOH, pyridine, 1,4-dioxane) were purchased from Acros Organics or Sigma-Aldrich and used as received. Solvents used for flash column chromatography were distilled before use. Commercially available reagents were used as supplied unless stated otherwise. Reactions were magnetically stirred and monitored by thin-layer chromatography (TLC) with silica gel 60-F254 aluminium plates from Merck. TLC plates were visualized by exposure to ultraviolet light (UV, $254 \mathrm{~nm}$ ) and/or exposure to an aqueous solution of potassium permanganate $\left(\mathrm{KMnO}_{4}\right)$, an aqueous solution of ceric ammonium molybdate (CAM), an ethanolic solution of vanillin or an ethanolic solution of 4-anisaldehyde followed by heating with a heat gun. Flash column chromatography was performed with silica gel $60 \mathrm{M}(40-63 \mu \mathrm{m})$ from Macherey-Nagel. Yields refer to chromatographically and spectroscopically pure compounds unless stated otherwise. NMR spectra were recorded on a $400 \mathrm{MHz}$ spectrometer (Avance III 400), a $600 \mathrm{MHz}$ spectrometer (Avance III 600) with and without cryoplatform or an $800 \mathrm{MHz}$ spectrometer (Avance NEO) with cryoplatform from Bruker. All NMR spectra were measured in $\mathrm{CDCl}_{3}$ solution and referenced to the residual $\mathrm{CHCl}_{3}$ signal $\left({ }^{1} \mathrm{H}, \delta=7.26 \mathrm{ppm},{ }^{13} \mathrm{C}, \delta=77.16 \mathrm{ppm}\right)$. All ${ }^{1} \mathrm{H}$ and ${ }^{13} \mathrm{C}$ shifts are given in ppm ( $\mathrm{s}=$ singlet, $\mathrm{d}=$ doublet, $\mathrm{t}=$ triplet, $\mathrm{q}=$ quartet, $\mathrm{m}=$ multiplet, $\mathrm{br}=$ broad signal). Assignments of proton resonance were confirmed, when possible, by correlated spectroscopy. High-resolution mass spectra were measured on an LTQ Orbitrap Velos spectrometer from Thermo Fisher Scientific (Velos Pro) with or without loop-mode injection from a Waters (RP18) HPLC system or a Micromass LCT spectrometer via loop-mode injection from a Waters (Alliance 2695) HPLC system. Ionization was achieved by ESI, modes of ionization, calculated, and found mass are given. IR spectra were measured on a PerkinEImer Spectrum 100 FT-IR spectrometer. Optical rotation values were measured on a Jasco P-2000 Digital Polarimeter using the sodium $D$ line $(589 \mathrm{~nm})$. X-ray diffraction analysis of single crystals was performed at $100 \mathrm{~K}$ on an STOE IPDS-II diffractometer equipped with a graphite-monochromated radiation source $(\lambda=0.71073 \AA)$ and an image plate detection system. A crystal mounted on a fine glass fibre with silicon grease was employed. 


\section{Total Synthesis of (+)-Pepluanol A}
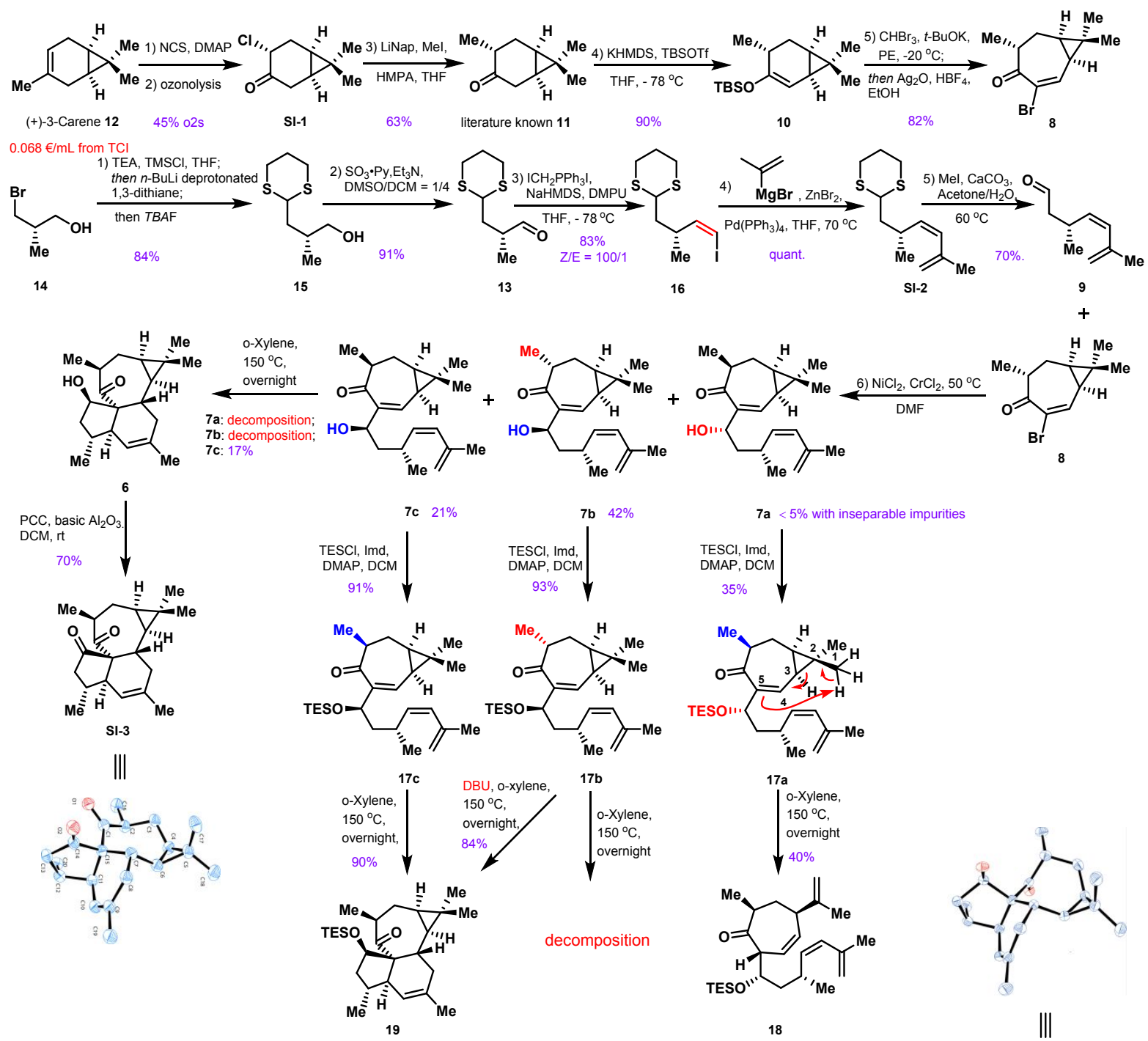

18

||
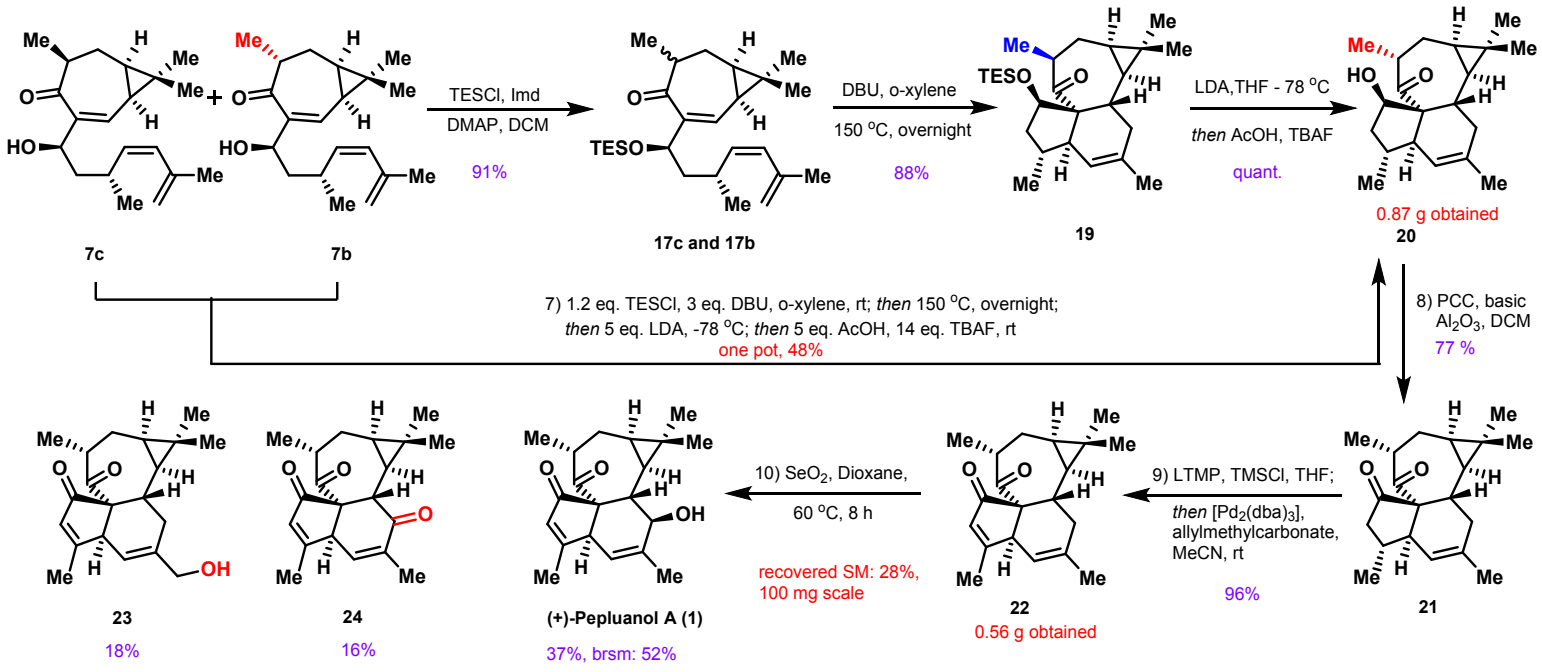


\section{Experimental Procedures and Characterization Data}

\section{Synthesis of 11}

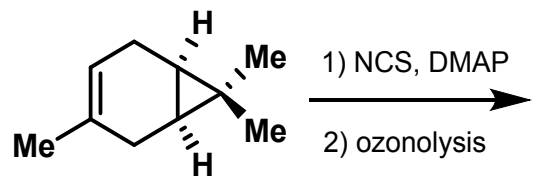

(+)-3-Carene 12 $0.068 € / \mathrm{mL}$ from TC

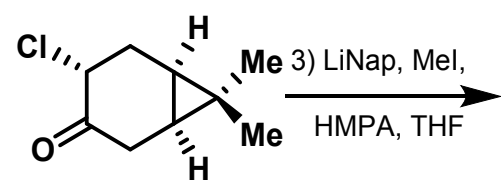

SI-1

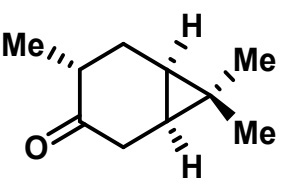

11

This three-step procedure was reported by Baran and co-workers ${ }^{[1]}$ and modified slightly.

The optical rotation of $(+)-3$-carene 12 was measured prior to use. $\left([\alpha]_{D}{ }^{4:}+16.3\right.$, neat; identical to the reported value by Tokyo Chemical Industry). To a solution of (+)-3-carene 12 (22.5 g, 165 mmol, 1.00 equiv) in $\mathrm{CH}_{2} \mathrm{Cl}_{2}(600 \mathrm{~mL})$ was added $\mathrm{N}$-chlorosuccinimide $(66.2 \mathrm{~g}, 496 \mathrm{mmol}, 3.00$ equiv) and 4-dimethylaminopyridine (2.02 g, $16.5 \mathrm{mmol}, 0.100$ equiv) and the solution was stirred at room temperature for $3 \mathrm{~h}$. Pentane $(600 \mathrm{~mL})$ was added, and the resulting suspension was stirred for $5 \mathrm{~min}$ before being filtered through a pad of Celite ${ }^{\circledR}$. The solution was concentrated under reduced pressure to give crude chloro-carene mixed with insoluble solid, which was diluted with pentane and filtered through a pad of Celite ${ }^{\circledR}$ again. After removal of solvent, the liquid chlorocarene was directly used in the next step without further purification since it decomposes on silica gel.

To a solution of crude chloro-carene in $\mathrm{CH}_{2} \mathrm{Cl}_{2}(400 \mathrm{~mL})$ was added $\mathrm{MeOH}(125 \mathrm{~mL})$ under $\mathrm{N}_{2}$, and the solution was cooled to $-78{ }^{\circ} \mathrm{C}$. Through the solution was bubbled $\mathrm{O}_{3}$ at $-78{ }^{\circ} \mathrm{C}$ until the solution turned deep-blue. Excess $\mathrm{O}_{3}$ was expelled by bubbling $\mathrm{O}_{2}$ through the solution until it turned colourless again. Thiourea $(21.0 \mathrm{~g}, 276 \mathrm{mmol})$ was added, and the reaction mixture was warmed to room temperature, and stirring was continued for $2 \mathrm{~h}$. The reaction mixture was washed with water $(2 \times 400 \mathrm{~mL})$ and brine $(400 \mathrm{~mL})$, and the combined organic layers were dried over $\mathrm{MgSO}_{4}$, filtered and carefully concentrated in vacuo. Purification of the residue by flash column chromatography (silica gel, a column packed in $\mathrm{CH}_{2} \mathrm{Cl}_{2}$, then pentane/Et $\mathrm{H}_{2} \mathrm{O}=20 / 1$ to 10/1) yielded the chloro-ketone SI-1 (13.0 g, 45\% over 2 steps) as a colourless liquid.

Preparation of Li-napthalenide solution: A $1 \mathrm{~L}$ three-neck flask was charged with naphthalene (23.8 g, $185 \mathrm{mmol}, 2.45$ equiv) and dry THF (460 mL). To this solution, freshly cut lithium (1.30 g, $185 \mathrm{mmol}, 2.45$ equiv) was added. The mixture was sonicated for $3 \mathrm{~h}$. 
A separate $2 \mathrm{~L}$ three-neck flask was charged with a solution of SI-1 $(13.0 \mathrm{~g}, 75.3 \mathrm{mmol}, 1.00$ equiv) in dry THF $(300 \mathrm{~mL})$ and cooled to $-78{ }^{\circ} \mathrm{C}$. The freshly prepared Li-napthalenide solution was slowly added over $40 \mathrm{~min}$ until the dark-green colour of the reaction mixture persisted for ca. $1 \mathrm{~min}$. A solution of HMPA (26.2 mL, $151 \mathrm{mmol}, 2.00$ equiv) and methyl iodide (23.4 mL, $377 \mathrm{mmol}, 5.00$ equiv) in THF $(50.0 \mathrm{~mL})$ was added over $15 \mathrm{~min}$, and the resulting reaction mixture was stirred at $-78{ }^{\circ} \mathrm{C}$ for $1 \mathrm{~h}$. Then the reaction was warmed up to room temperature gradually by removal of cooling bath, and stirring was continued for $20 \mathrm{~min}$. Then water $(400 \mathrm{~mL})$ was added, and the organic layer was separated, and the aqueous layer was extracted with $\mathrm{Et}_{2} \mathrm{O}(3 \times 500$ $\mathrm{mL}$ ). The combined organic layers were dried over $\mathrm{MgSO}_{4}$, filtered and carefully concentrated under reduced pressure. During concentration, naphthalene separated out as crystalline solid, filtered through a short pad of silica gel and washed with pentane. After removal of the solvent under reduced pressure again, the crude product was purified by flash column chromatography (silica gel, eluting with pentane, then pentane/ $\mathrm{Et}_{2} \mathrm{O}=80: 1$ to $10: 1$ ) to afford methyl ketone 11 (8.50 g mixed with $\mathrm{Et}_{2} \mathrm{O}$, which contains $7.20 \mathrm{~g}$ product after calculation based on ${ }^{1} \mathrm{H} \mathrm{NMR}, 63 \%$ ) as a light yellow solution.

${ }^{1} \mathrm{H}$ NMR $\left(400 \mathrm{MHz}, \mathrm{CDCl}_{3}\right.$ ): $\delta 2.57$ (dd, $\left.J=18.3,8.5 \mathrm{~Hz}, 1 \mathrm{H}\right), 2.23$ (qdd, $J=7.2,4.8,2.6 \mathrm{~Hz}, 1 \mathrm{H}$ ), 2.09 (dd, $J=18.3,3.4 \mathrm{~Hz}, 1 \mathrm{H}$ ), 2.02 (dddd, $J=15.0,9.0,2.5,0.9 \mathrm{~Hz}, 1 \mathrm{H}$ ), 1.72 (ddd, $J=15.0$, $6.2,4.8 \mathrm{~Hz}, 1 \mathrm{H}), 1.23(\mathrm{~d}, J=7.2 \mathrm{~Hz}, 3 \mathrm{H}), 1.06(\mathrm{~s}, 3 \mathrm{H}), 1.04(\mathrm{dd}, J=8.7,3.4 \mathrm{~Hz}, 1 \mathrm{H}), 0.92(\mathrm{~s}, 3 \mathrm{H})$, $0.86-0.80(\mathrm{~m}, 1 \mathrm{H})$ ppm; $\left(\mathrm{Et}_{2} \mathrm{O}\right.$ signals excluded $)$

${ }^{13} \mathrm{C}$ NMR $\left(100 \mathrm{MHz}, \mathrm{CDCl}_{3}\right)$ : $\delta 218.4,41.8,34.3,28.2,27.0,21.9,19.7,17.2,16.9,14.9$ ppm; $\left(\mathrm{Et}_{2} \mathrm{O}\right.$ signals excluded)

HRMS (ESI): $\mathrm{m} / \mathrm{z}$ calcd for $\mathrm{C}_{10} \mathrm{H}_{17} \mathrm{O}^{+}[M+\mathrm{H}]^{+}:$153.1274, found: 153.1272 .

$[\alpha]_{D}^{5:}+82.9\left(\right.$ c 1.1, $\left.\mathrm{CHCl}_{3}\right)$; Baran's result ${ }^{[1 \mathrm{~b}]}[\alpha]_{\mathrm{D}}=+67.0$ (c 1.0, $\mathrm{CHCl}_{3}$ )

This spectral data was identical to previous literature. ${ }^{[1]}$

\section{Synthesis of 10}

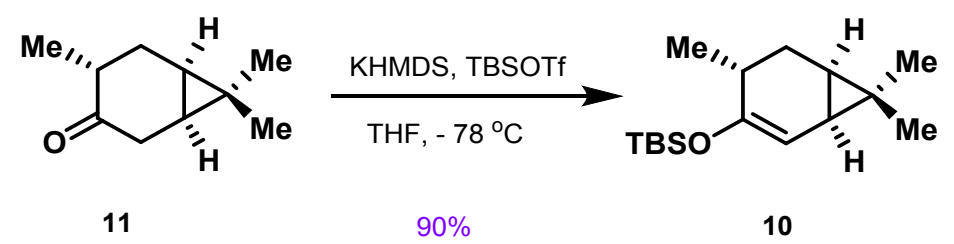

The ketone 11 (4.02 g, $26.4 \mathrm{mmol}, 1.00$ equiv) was dissolved in dry THF (106 mL, $0.25 \mathrm{M}$ ) and cooled to $-78^{\circ} \mathrm{C}$. Then potassium bis(trimethylsilyl)amide solution in toluene $(52.9 \mathrm{~mL}, 0.50 \mathrm{M}$ in 
toluene, 1.00 equiv) was added dropwise to the above solution and let the resultant mixture stir for $20 \mathrm{~min}$ at $-78^{\circ} \mathrm{C}$. Afterwards, to the formed enolate solution was added TBS-triflate $(6.00 \mathrm{~mL}$, $26.4 \mathrm{mmol}, 1.00$ equiv) dropwise. After $15 \mathrm{~min}$, the reaction was quenched by aq. $\mathrm{NH}_{4} \mathrm{Cl}$ and extracted with $\mathrm{Et}_{2} \mathrm{O} \times 3$. The combined organic layers were dried over $\mathrm{MgSO}_{4}$ and concentrated under reduced pressure. The crude residue was purified by flash column chromatography on silica gel (pure PE) to yield TBS-enolate $10(6.33 \mathrm{~g}, 90 \%)$ as a colourless oil.

$\boldsymbol{R}_{\mathrm{f}}: 0.78$ (PE, CAM);

${ }^{1} \mathrm{H}$ NMR $\left(400 \mathrm{MHz}, \mathrm{CDCl}_{3}\right): \delta 4.74(\mathrm{~d}, \mathrm{~J}=3.6 \mathrm{~Hz}, 1 \mathrm{H}), 1.88-1.80(\mathrm{~m}, 1 \mathrm{H}), 1.79-1.67(\mathrm{~m}, 2 \mathrm{H})$, $1.11(\mathrm{~d}, J=6.8 \mathrm{~Hz}, 3 \mathrm{H}), 1.05(\mathrm{~s}, 3 \mathrm{H}), 1.00-0.96(\mathrm{~m}, 1 \mathrm{H}), 0.91(\mathrm{~s}, 12 \mathrm{H}), 0.73(\mathrm{td}, J=8.6,5.3 \mathrm{~Hz}$, $1 \mathrm{H}), 0.13(\mathrm{~s}, 3 \mathrm{H}), 0.11(\mathrm{~s}, 3 \mathrm{H}) \mathrm{ppm}$;

${ }^{13} \mathrm{C}$ NMR $\left(100 \mathrm{MHz}, \mathrm{CDCl}_{3}\right): \delta 157.6,100.4,33.4,28.0,27.9,25.9,23.5,23.3,19.9,18.1,17.6$, 15.5, -4.3 ppm;

HRMS (ESI): $\mathrm{m} / \mathrm{z}$ calcd for $\mathrm{C}_{18} \mathrm{H}_{34} \mathrm{ONSi}^{+}\left[\mathrm{M}+\mathrm{CH}_{3} \mathrm{CN}+\mathrm{H}\right]^{+}: 308.2404$, found: 308.2402 ;

IR (neat sample): 2959, 2930, 2860, 1654, 1462, 1375, 1253, 1194, 912, 837, 778, $688 \mathrm{~cm}^{-1}$;

$[\alpha]_{D}^{4:}+38.1\left(c 1.1, \mathrm{CHCl}_{3}\right)$.

\section{Synthesis of 8}

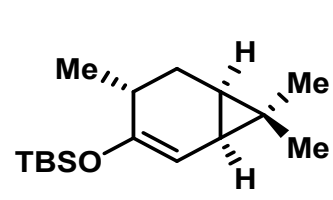

10

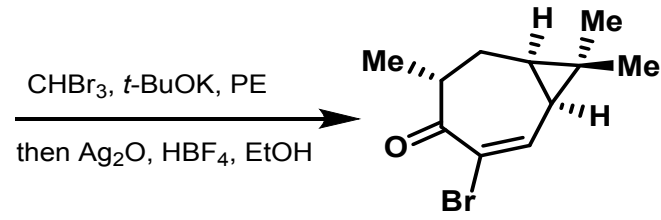

$82 \%$

8

TBS enol ether 10 (8.90 g, $33.4 \mathrm{mmol}, 1.00$ equiv) was dissolved in roti-distilled petroleum ether $(670 \mathrm{~mL}, 0.05 \mathrm{M})$. The solution was cooled to $-20^{\circ} \mathrm{C}$ followed by one-portion addition of potassium tert-butoxide ( $28.1 \mathrm{~g}, 251 \mathrm{mmol}, 7.50$ equiv). Then to the above stirring suspension was added dropwise bromoform (14.6 mL, $167 \mathrm{mmol}, 5.00$ equiv). After $10 \mathrm{~min}$, the reaction mixture was filtered through a short silica gel pad and washed with petroleum ether. Then the solvent was removed under reduced pressure to give crude residue as a yellow liquid. (PE, $\left.R_{\mathrm{f}}=0.84, \mathrm{CAM}\right)$ Without further purification, the crude residue was re-dissolved in $\mathrm{EtOH}(335 \mathrm{~mL}, 0.10 \mathrm{M})$, and the resultant solution was cooled to $0{ }^{\circ} \mathrm{C}$. Then silver oxide $(10.0 \mathrm{~g}, 43.0 \mathrm{mmol}, 1.30$ equiv) was added in one portion, and the resulting suspension was stirred vigorously followed by slow addition of tetrafluoroboric acid solution (48 wt. \% in $\mathrm{H}_{2} \mathrm{O}, 43.0 \mathrm{~mL}, 330 \mathrm{mmol}, 10.0$ equiv). Afterwards, the 
cooling bath was removed, and the stirring was continued for 3 hours at room temperature. Then the stirring mixture was re-cooled to $0{ }^{\circ} \mathrm{C}$, the saturated aq. $\mathrm{NaHCO}_{3}$ was added carefully to neutralize the reaction until no $\mathrm{CO}_{2}$ forming. After adding some brine, the mixture was extracted with $\mathrm{Et}_{2} \mathrm{O}(500 \mathrm{~mL} \times 3)$. The organic layers were combined, dried over $\mathrm{MgSO}_{4}$, filtered and concentrated in vacuo. The resultant residue was purified by flash column chromatography on silica gel $(P E / E A=20 / 1)$ to yield 7-membered ketone $8(6.63 \mathrm{~g}, 82 \%)$ as a slight-yellow oil.

$\boldsymbol{R}_{\mathbf{f}}: 0.58$ (PE/EA = 10:1, CAM);

${ }^{1} \mathrm{H}$ NMR (400 MHz, $\mathrm{CDCl}_{3}$ ) $\delta 7.06$ (d, $J=3.4 \mathrm{~Hz}, 1 \mathrm{H}$ ), 2.92 (qdd, $J=7.3,4.7,2.4 \mathrm{~Hz}, 1 \mathrm{H}$ ), 1.95 (ddd, $J=15.1,7.3,4.7 \mathrm{~Hz}, 1 \mathrm{H}$ ), 1.73 (ddd, $J=15.1,10.4,2.4 \mathrm{~Hz}, 1 \mathrm{H}), 1.35$ (d, J = $7.4 \mathrm{~Hz}, 3 \mathrm{H}$ ), $1.30(\mathrm{dd}, J=8.4,3.4 \mathrm{~Hz}, 1 \mathrm{H}), 1.23-1.20(\mathrm{~m}, 1 \mathrm{H}), 1.19(\mathrm{~s}, 3 \mathrm{H}), 1.07$ (s, 3H) ppm;

${ }^{13} \mathrm{C}$ NMR $\left(100 \mathrm{MHz}, \mathrm{CDCl}_{3}\right) \delta$ 200.2, 143.7, 125.0, 45.9, 29.7, 28.0, 25.5, 25.1, 25.0, 16.2, 15.8 ppm;

HRMS (ESI): $\mathrm{m} / \mathrm{z}$ calcd for $\mathrm{C}_{11} \mathrm{H}_{16} \mathrm{BrO}^{+}[\mathrm{M+H}]^{+}:$243.0379, found: 234.0379;

IR (neat sample): 2930, 2867, 1671, 1596, 1456, 1377, 1342, 1133, 985, 899, 843, $804 \mathrm{~cm}^{-1}$; $[\alpha]_{D}^{4:}+272\left(c 0.7, \mathrm{CHCl}_{3}\right)$.

\section{Synthesis of 15}

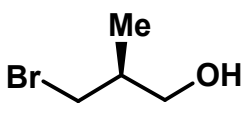

14
1) $\mathrm{Et}_{3} \mathrm{~N}, \mathrm{TMSCl}, \mathrm{THF}$; then $n$-BuLi deprotonated 1,3-dithiane; then TBAF

$84 \%$

15

Commercially available (S)-3-bromo-2-methylpropan-1-ol 14 (500 mg, 3.27 mmol, 1.00 equiv) was dissolved in THF $(16.4 \mathrm{~mL}, 0.20 \mathrm{M})$ at $0{ }^{\circ} \mathrm{C}$. To above stirring solution were added triethylamine $(0.600 \mathrm{~mL}, 4.25 \mathrm{mmol}, 1.30$ equiv) and TMSCI (0.500 mL, $3.92 \mathrm{mmol}, 1.20$ equiv). The resulting suspension was stirred at room temperature for $15 \mathrm{~min}$. In parallel, to an additional flask loaded with 1,3-dithiane (982 $\mathrm{mg}, 8.17 \mathrm{mmol}, 2.50$ equiv) in THF (10.0 mL) was added dropwise $n$-BuLi (3.20 mL, $8.00 \mathrm{mmol}, 2.5 \mathrm{M}$ in hexane, 2.45 equiv) at $0{ }^{\circ} \mathrm{C}$. Stirring was continued for $15 \mathrm{~min}$ at that temperature. Then the resulting deprotonated 1,3-dithiane solution was slowly transferred to the above solution via cannula at $0{ }^{\circ} \mathrm{C}$. The reaction was kept stirring for $20 \mathrm{~min}$ at $0{ }^{\circ} \mathrm{C}$ before TBAF $(9.80 \mathrm{~mL}, 9.80 \mathrm{mmol}, 1.0 \mathrm{M}$ in THF, 3.00 equiv) was added dropwise. The reaction was quenched by aq. $\mathrm{NH}_{4} \mathrm{Cl}$ till TLC shows the reaction is done (about $30 \mathrm{~min}$ at $0{ }^{\circ} \mathrm{C}$ ). 
Then the reaction was diluted with $\mathrm{Et}_{2} \mathrm{O}$, extracted with $\mathrm{Et}_{2} \mathrm{O}$ three time. The combined organic layers were dried over $\mathrm{MgSO}_{4}$, filtered and evaporated under reduced pressure. The remaining residue was purified by column chromatography on silica gel (PE/EA $=5 / 1$ to $3 / 1$ ), yielding alcohol $15(526 \mathrm{mg}, 84 \%)$ as a colourless oil.

$\mathbf{R}_{\mathbf{f}}=0.19(\mathrm{PE} / \mathrm{EA}=5 / 1, \mathrm{CAM})$;

${ }^{1} \mathrm{H}$ NMR (400 MHz, $\left.\mathrm{CDCl}_{3}\right) \delta 4.13$ (dd, J = 8.3, $\left.6.5 \mathrm{~Hz}, 1 \mathrm{H}\right), 3.50$ (dd, J = 6.2, $2.6 \mathrm{~Hz}, 2 \mathrm{H}$ ), $2.95-$ $2.73(\mathrm{~m}, 4 \mathrm{H}), 2.17-2.07(\mathrm{~m}, 1 \mathrm{H}), 2.07-1.95(\mathrm{~m}, 1 \mathrm{H}), 1.92-1.77(\mathrm{~m}, 2 \mathrm{H}), 1.58$ (ddd, J = 14.2, 7.9, $6.5 \mathrm{~Hz}, 1 \mathrm{H}), 1.51$ (brs, $1 \mathrm{H}), 0.97$ (d, J = 6.8 Hz, 3H) ppm;

${ }^{13} \mathrm{C}$ NMR $\left(100 \mathrm{MHz}, \mathrm{CDCl}_{3}\right) \delta 67.9,45.5,39.3,33.0,30.6,30.5,26.1,16.8$ ppm;

HRMS (ESI): $\mathrm{m} / \mathrm{z}$ calcd for $\mathrm{C}_{8} \mathrm{H}_{15} \mathrm{~S}_{2}{ }^{+}\left[\mathrm{M}-\mathrm{H}_{2} \mathrm{O}+\mathrm{H}\right]^{+}:$: 175.0610, found: 175.0609;

IR (neat sample): 3404, 2900, 1422, 1275, 1042, 983, 906, 764, $665 \mathrm{~cm}^{-1}$;

$[\alpha]_{\mathrm{D}}^{4}:+10.9\left(c \mathrm{c} 0.75, \mathrm{CHCl}_{3}\right)$.

\section{Synthesis of 13}

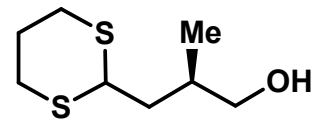

15

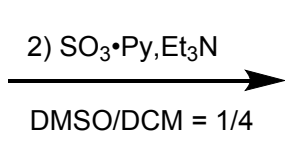

$91 \%$

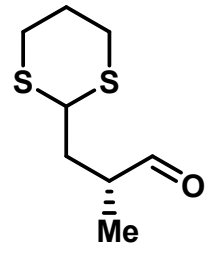

13

To a stirring solution of alcohol 15 (6.00 g, $31.2 \mathrm{mmol}, 1.00$ equiv) in DMSO/DCM (1/4, $312 \mathrm{~mL}$, $0.10 \mathrm{M})$ were added triethylamine $(17.4 \mathrm{~mL}, 125 \mathrm{mmol}, 4.00$ equiv) and sulfur trioxide pyridine complex ( $9.93 \mathrm{~g}, 62.4 \mathrm{mmol}, 2.00$ equiv) at $0{ }^{\circ} \mathrm{C}$. Afterwards, the cooling bath was removed to let the reaction warm to room temperature. $1 \mathrm{~h}$ later, the reaction was quenched by saturated aq. $\mathrm{NH}_{4} \mathrm{Cl}$ and diluted with DCM. The mixture was extracted with DCM three times and washed with brine once. The organic layers were separated, dried over $\mathrm{MgSO}_{4}$, filtered and concentrated under reduced pressure. The resulting residue was purified by flash column chromatography on silica gel with eluent $(P E / E A=8 / 1)$, yielding aldehyde $13(5.40 \mathrm{~g}, 91 \%)$ as a pale-yellow oil.

$\mathbf{R}_{\mathrm{f}}=0.41(\mathrm{PE} / \mathrm{EA}=8 / 1, \mathrm{CAM})$;

${ }^{1} \mathrm{H}$ NMR $\left(400 \mathrm{MHz}, \mathrm{CDCl}_{3}\right) \delta 9.72-9.44(\mathrm{~m}, 1 \mathrm{H}), 4.06(\mathrm{t}, \mathrm{J}=7.5 \mathrm{~Hz}, 1 \mathrm{H}), 2.88-2.80(\mathrm{~m}, 4 \mathrm{H})$, $2.74(\mathrm{hd}, J=6.9,1.4 \mathrm{~Hz}, 1 \mathrm{H}), 2.31-2.18(\mathrm{~m}, 1 \mathrm{H}), 2.15-2.05(\mathrm{~m}, 1 \mathrm{H}), 1.94-1.81(\mathrm{~m}, 1 \mathrm{H}), 1.74$ (dt, $J=14.2,6.9 \mathrm{~Hz}, 1 \mathrm{H}), 1.15(\mathrm{dd}, J=7.2,0.6 \mathrm{~Hz}, 3 \mathrm{H}) \mathrm{ppm}$; 
${ }^{13} \mathrm{C}$ NMR $\left(100 \mathrm{MHz}, \mathrm{CDCl}_{3}\right) \delta 203.6,44.8,43.6,36.1,30.1,30.0,25.9,13.8$ ppm;

HRMS (ESI): $\mathrm{m} / \mathrm{z}$ calcd for $\mathrm{C}_{8} \mathrm{H}_{15} \mathrm{OS}_{2}{ }^{+}[\mathrm{M}+\mathrm{H}]^{+:}$191.0559, found: 191.0560;

IR (neat sample): 2898, 1720, 1455, 1421, 1275, 1243, 906, $765 \mathrm{~cm}^{-1}$;

$[\alpha]_{D}^{4:}+23.6\left(c 0.9, \mathrm{CHCl}_{3}\right)$.

\section{Synthesis of 16}

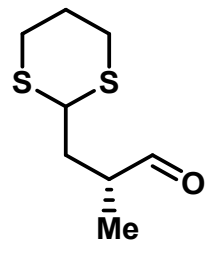

13

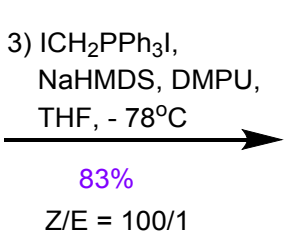

$Z / E=100 / 1$

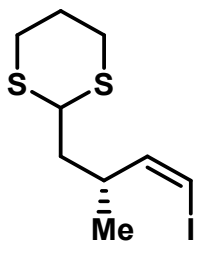

16

A suspension of iodomethyltriphenylphosphonium iodide ${ }^{[2]}(15.0 \mathrm{~g}, 28.4 \mathrm{mmol}, 1.20$ equiv) in THF (95.0 mL) was prepared and sodium bis(trimethylsilyl)amide (28.4 mL, 1.0 M in THF, 28.4 mmol, 1.20 equiv) was added at rt. The mixture was stirred at room temperature for $10 \mathrm{~min}$ and cooled to $-78{ }^{\circ} \mathrm{C}$ before DMPU $(7.10 \mathrm{~mL}, 59.1 \mathrm{mmol}, 2.50$ equiv) was added. The mixture was allowed to stir for another $10 \mathrm{~min}$. The solution of aldehyde 13 (4.50 g, $23.6 \mathrm{mmol}, 1.00$ equiv) in THF $(79.0 \mathrm{~mL}, 0.30 \mathrm{M})$ was cooled to $-78^{\circ} \mathrm{C}$ and added via cannula covered with cotton, and the reaction was stirred at $-78{ }^{\circ} \mathrm{C}$ for $15 \mathrm{~min}$. The mixture was then diluted with $\mathrm{Et}_{2} \mathrm{O}$ and filtered over silica, and the filter cake was washed with EA. The combined filtrates were washed with brine to clear the suspension and dried over $\mathrm{MgSO}_{4}$. After removal of the solvent under reduced pressure, the crude product was purified by flash column chromatography on silica gel $(P E / E A=20 / 1)$ to give intermediate $(Z)$-vinyl iodide 16 (6.17 g, 83\%, inseparable geometric isomer Z/E = 100/1) as a pale-yellow oil.

$\mathbf{R}_{\mathbf{f}}=0.48(\mathrm{PE} / \mathrm{EA}=20 / 1, \mathrm{CAM})$;

${ }^{1} \mathrm{H}$ NMR $\left(400 \mathrm{MHz}, \mathrm{CDCl}_{3}\right) \delta 6.22(\mathrm{dd}, J=7.4,0.7 \mathrm{~Hz}, 1 \mathrm{H}), 5.96(\mathrm{dd}, J=8.9,7.4 \mathrm{~Hz}, 1 \mathrm{H}), 3.93$ (dd, $J=7.7,6.6 \mathrm{~Hz}, 1 \mathrm{H}), 2.94-2.78(\mathrm{~m}, 5 \mathrm{H}), 2.10(\mathrm{dtt}, J=14.0,4.8,3.0 \mathrm{~Hz}, 1 \mathrm{H}), 1.96-1.79(\mathrm{~m}$, $1 \mathrm{H}$ ), 1.76 (ddd, $J=7.9,6.5,1.4 \mathrm{~Hz}, 2 \mathrm{H}), 1.03$ (d, $J=6.8 \mathrm{~Hz}, 3 \mathrm{H}$ ) ppm;

${ }^{13} \mathrm{C}$ NMR $\left(100 \mathrm{MHz}, \mathrm{CDCl}_{3}\right) \delta$ 145.6, 81.9, 45.1, 41.6, 36.8, 30.5, 30.3, 26.1, 19.2 ppm;

HRMS (ESI): $\mathrm{m} / \mathrm{z}$ calcd for $\mathrm{C}_{9} \mathrm{H}_{16} \mathrm{IS}_{2}{ }^{+}[M+\mathrm{H}]^{+}:$314.9733, found: 314.9731;

IR (neat sample): 2957, 2930, 2897, 1421, 1275, 907, $710 \mathrm{~cm}^{-1}$;

$[\alpha]_{D}^{4:}+61.7\left(c 1.5, \mathrm{CHCl}_{3}\right)$. 


\section{Synthesis of SI-2}

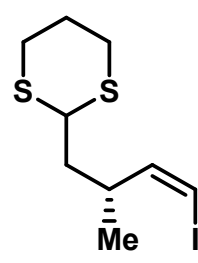

16

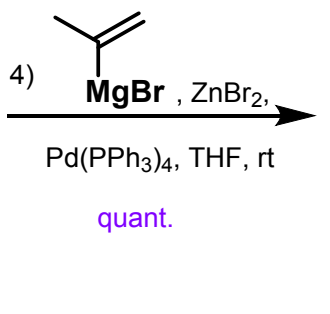

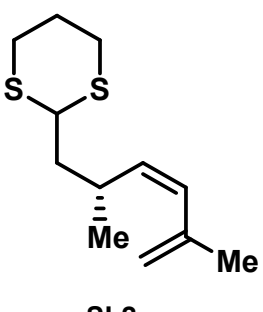

SI-2

Zinc bromide (8.82 g, $39.1 \mathrm{mmol}, 2.00$ equiv) was dissolved in THF (117 mL), and a solution of isopropenylmagnesium bromide $(78.2 \mathrm{~mL}, 39.1 \mathrm{mmol}, 0.50 \mathrm{M}$ in THF, 2.00 equiv) was added dropwise at $0{ }^{\circ} \mathrm{C}$. After $10 \mathrm{~min}$ at room temperature, the reaction was cooled to $0{ }^{\circ} \mathrm{C}$ again. The (Z)-vinyl iodide 16 (6.15 g, $19.6 \mathrm{mmol}, 1.00$ equiv) in THF (98.0 mL, $0.20 \mathrm{M})$ was cannulated into the above mixture. Then $\left[\mathrm{Pd}\left(\mathrm{PPh}_{3}\right)_{4}\right](904 \mathrm{mg}, 0.783 \mathrm{mmol}, 0.0400$ equiv) was added in one portion, and the mixture was warmed to room temperature and stirred for an additional hour. The reaction was cooled to $0{ }^{\circ} \mathrm{C}$ again and quenched by the addition of saturated aqueous $\mathrm{NH}_{4} \mathrm{Cl}$, diluted with water and $\mathrm{Et}_{2} \mathrm{O}$, layers separated, the aqueous phase extracted with $\mathrm{Et}_{2} \mathrm{O}$, organic extracts combined, washed with brine, filtered through $\mathrm{MgSO}_{4}$, concentrated in vacuo, and purified by flash column chromatography on silica gel (PE/EA = 30:1) to yield diene SI-2 (4.47 g, quant.) as a colourless oil.

$\mathbf{R}_{\mathrm{f}}=0.55(\mathrm{PE} / \mathrm{EA}=20 / 1, \mathrm{CAM})$;

${ }^{1} \mathrm{H}$ NMR $\left(400 \mathrm{MHz}, \mathrm{CDCl}_{3}\right) \delta 5.86(\mathrm{~d}, J=11.7,1.0 \mathrm{~Hz}, 1 \mathrm{H}), 5.11(\mathrm{dd}, J=11.7,10.3 \mathrm{~Hz}, 1 \mathrm{H}), 4.90$ (dddd, $J=19.4,2.5,1.5,0.8 \mathrm{~Hz}, 2 \mathrm{H}), 3.95$ (dd, $J=8.8,5.7 \mathrm{~Hz}, 1 \mathrm{H}), 3.23-3.06(\mathrm{~m}, 1 \mathrm{H}), 2.93-$ $2.75(\mathrm{~m}, 4 \mathrm{H}), 2.09$ (dtt, $J=15.1,4.7,2.8 \mathrm{~Hz}, 1 \mathrm{H}), 1.91(\mathrm{~s}, 3 \mathrm{H}), 1.90-1.79(\mathrm{~m}, 1 \mathrm{H}), 1.75-1.58$ $(\mathrm{m}, 2 \mathrm{H}), 1.00(\mathrm{~d}, J=6.6 \mathrm{~Hz}, 3 \mathrm{H}) \mathrm{ppm}$;

${ }^{13} \mathrm{C}$ NMR $\left(100 \mathrm{MHz}, \mathrm{CDCl}_{3}\right) \delta 142.1,135.9,131.2,115.7,45.6,43.3,30.6,30.3,29.7,26.2,23.3$, $21.4 \mathrm{ppm}$;

HRMS (ESI): $\mathrm{m} / \mathrm{z}$ calcd for $\mathrm{C}_{12} \mathrm{H}_{21} \mathrm{~S}_{2}{ }^{+}[M+\mathrm{H}]^{+}:$229.1079, found: 229.1080;

IR (neat sample): 2954, 2897, 1635, 1455, 1422, 1371, 1275, 1242, 1181, 890, $766 \mathrm{~cm}^{-1}$;

$[\alpha]_{D}^{4}:+3.90\left(c 1.1, \mathrm{CHCl}_{3}\right)$. 


\section{Synthesis of 9}

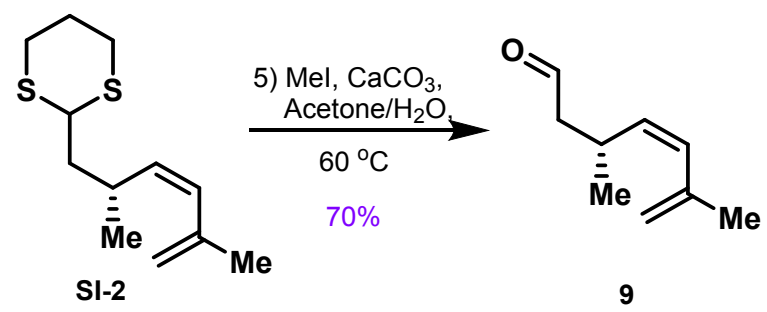

Diene SI-2 (4.03 g, $17.7 \mathrm{mmol}, 1.00$ equiv) was dissolved in acetone and water (4/1, $88.0 \mathrm{~mL}$, $0.20 \mathrm{M}) . \mathrm{CaCO}_{3}(26.5 \mathrm{~g}, 265 \mathrm{mmol}, 15.0$ equiv) and Mel (32.0 mL, $530 \mathrm{mmol}, 30.0$ equiv) were added to the above solution at room temperature. Then the flask was charged with a condenser, and the reaction was stirred vigorously and heated to $60{ }^{\circ} \mathrm{C}$. After $3 \mathrm{~h}$, the reaction was filtrated and washed with $\mathrm{Et}_{2} \mathrm{O}$, and the resulting filtrate was diluted with water and $\mathrm{Et}_{2} \mathrm{O}$. Then the mixture was extracted with $\mathrm{Et}_{2} \mathrm{O}$ three times, and the combined organic layers were washed with brine and dried over $\mathrm{MgSO}_{4}$ and concentrated under reduced pressure (700 millibar). The crude product was purified by flash column chromatography on silica gel with eluent (pentane/Et $\mathrm{t}_{2} \mathrm{O}=$ $15 / 1)$ to give volatile aldehyde 9 ( $1.70 \mathrm{~g}, 70 \%, 700$ millibar to give product $1.70 \mathrm{~g}$ in $1.7 \mathrm{~g} \mathrm{Et}_{2} \mathrm{O}$ )

$\mathbf{R}_{\mathbf{f}}=0.5(\mathrm{PE} / \mathrm{EA}=10 / 1, \mathrm{CAM}) ;$

${ }^{1} \mathrm{H}$ NMR $\left(400 \mathrm{MHz}, \mathrm{CDCl}_{3}\right) \delta 9.68(\mathrm{t}, J=2.3 \mathrm{~Hz}, 1 \mathrm{H}), 5.82(\mathrm{~d}, J=11.7 \mathrm{~Hz}, 1 \mathrm{H}), 5.20$ (dd, $J=11.7$, $10.3 \mathrm{~Hz}, 1 \mathrm{H}), 4.96(\mathrm{~s}, 1 \mathrm{H}), 4.84(\mathrm{~s}, 1 \mathrm{H}), 3.42-3.30(\mathrm{~m}, 1 \mathrm{H}), 2.37(\mathrm{dd}, J=7.0,2.4 \mathrm{~Hz}, 2 \mathrm{H}), 1.86$ (s, 3H), 1.06 (d, J = 6.7 Hz, 3H) ppm;

${ }^{13} \mathrm{C}$ NMR (100 MHz, $\left.\mathrm{CDCl}_{3}\right) \delta 202.3,141.6,135.0,130.8,115.6,51.2,27.8,23.4,21.6$ ppm;

HRMS (ESI): $\mathrm{m} / \mathrm{z}$ calcd for $\mathrm{C}_{9} \mathrm{H}_{15} \mathrm{O}^{+}[M+\mathrm{H}]^{+:}$: 139.1117, found: 139.1116;

IR (neat sample): 2969, 2718, 1723, 1635, 1455, 1373, 892, $780 \mathrm{~cm}^{-1}$;

$[\alpha]_{\mathrm{D}}^{4}:-113.4\left(c\right.$ 1.0, $\left.\mathrm{CHCl}_{3}\right)$. 


\section{Syntheses of $7 a, 7 b$ and $7 c$}

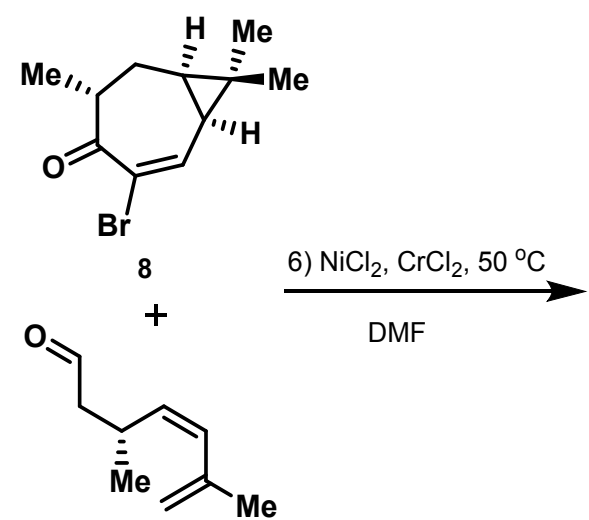

9

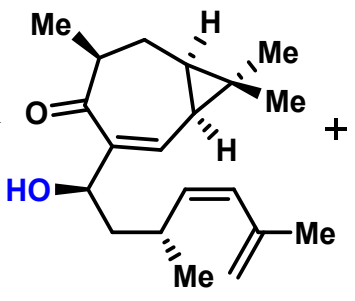

7c $21 \%$

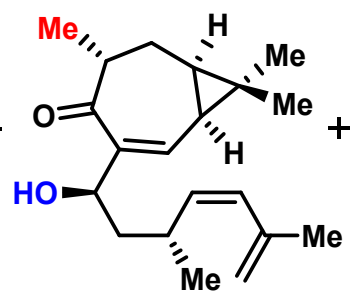

7b $42 \%$

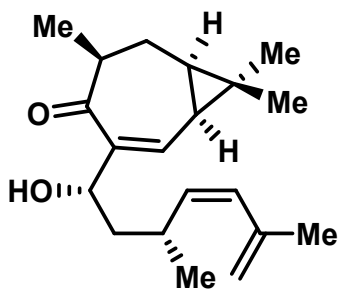

$7 \mathbf{a}<5 \%$ with impurities

Nickle dichloride (139 mg, $1.08 \mathrm{mmol}, 0.100$ equiv) and chromium dichloride (10.5 g, $86.0 \mathrm{mmol}$, 8.00 equiv) were added in an oven-dry flash in the glovebox. After putting the flask under nitrogen and in a room temperature water bath, the degassed DMF $(53.0 \mathrm{~mL})$ was added slowly to the stirring mixture. (Note: Trace of dimethylamine in DMF needs to be removed by bubbling nitrogen overnight. Otherwise, it makes the reaction fruitless.) The stirring was continued for 10 min at room temperature. Then the solution of 7-membered vinyl bromide 8 (5.23 g, $21.5 \mathrm{mmol}, 2.00$ equiv) and aldehyde 9 (1.50 g, $10.8 \mathrm{mmol}, 1.00$ equiv) in degassed DMF (53.0 mL) was added to the above blue mixture at room temperature. Afterwards, the reaction was allowed to warm to $50{ }^{\circ} \mathrm{C}$ and stirred for $1 \mathrm{~h}$. Then the dark blue mixture was cooled to room temperature, quenched by water carefully and diluted with $\mathrm{Et}_{2} \mathrm{O}$. The layers were separated, and the aqueous layer was extracted with $\mathrm{Et}_{2} \mathrm{O} \times 4$. The combined organic layers were washed with brine, dried over $\mathrm{MgSO}_{4}$, filtered and concentrated in vacuo. The resultant residue was submitted to a long column packed with silica gel with PE/EA (30/1 to $25 / 1$ to $20 / 1)$ as eluent to afford $7 \mathrm{c}(0.680 \mathrm{~g}, 21 \%$, slightly yellow oil), $7 \mathbf{b}(1.38 \mathrm{~g}, 42 \%$, slightly yellow oil) and 7a (less than $5 \%$ with inseparable impurities, slightly yellow oil), respectively.

7a: (After protection with TES and deprotection of TES procedure, we got a relatively clean sample for NMR in which contains inseparable E-geometric isomer on the sidechain.)

$\boldsymbol{R}_{\mathrm{f}}=0.48(\mathrm{PE} / \mathrm{EA}=5: 1, \mathrm{CAM})$;

${ }^{1} \mathrm{H}$ NMR $\left(400 \mathrm{MHz}, \mathrm{CDCl}_{3}\right) \delta 6.29(\mathrm{~d}, J=2.7 \mathrm{~Hz}, 1 \mathrm{H}), 5.83(\mathrm{~d}, J=11.7 \mathrm{~Hz}, 1 \mathrm{H}), 5.14(\mathrm{dd}, J=11.7$, $10.4 \mathrm{~Hz}, 1 \mathrm{H}), 4.88(\mathrm{~d}, J=17.6 \mathrm{~Hz}, 2 \mathrm{H}), 4.15(\mathrm{td}, J=8.6,4.6 \mathrm{~Hz}, 1 \mathrm{H}), 3.18(\mathrm{~d}, J=8.3 \mathrm{~Hz}, 1 \mathrm{H})$, $3.05-2.91(\mathrm{~m}, 1 \mathrm{H}), 2.69(\mathrm{dqd}, J=11.6,7.1,2.3 \mathrm{~Hz}, 1 \mathrm{H}), 1.88(\mathrm{t}, J=1.1 \mathrm{~Hz}, 3 \mathrm{H}), 1.87-1.82(\mathrm{~m}$, 1H), $1.65-1.57(\mathrm{~m}, 1 \mathrm{H}), 1.45$ (ddd, $J=14.4,11.5,10.0 \mathrm{~Hz}, 1 \mathrm{H}), 1.35$ (ddd, $J=13.5,8.7,4.7 \mathrm{~Hz}$, 
1H), $1.26(\mathrm{dd}, J=8.6,2.8 \mathrm{~Hz}, 1 \mathrm{H}), 1.24-1.18(\mathrm{~m}, 1 \mathrm{H}), 1.13(\mathrm{~s}, 3 \mathrm{H}), 1.09(\mathrm{~d}, J=7.1 \mathrm{~Hz}, 3 \mathrm{H}), 1.00$ (s, 3H), $0.99(\mathrm{~d}, \mathrm{~J}=6.7 \mathrm{~Hz}, 3 \mathrm{H}) \mathrm{ppm}$;

${ }^{13} \mathrm{C}$ NMR $\left(100 \mathrm{MHz}, \mathrm{CDCl}_{3}\right) \delta 211.2,143.2,142.1,138.6,136.9,130.4,115.3,74.1,47.1,44.8$, 29.5, 29.5, 29.3, 28.0, 27.2, 23.7, 23.4, 21.6, 17.9, 15.8 ppm;

HRMS (ESI): $\mathrm{m} / \mathrm{z}$ calcd for $\mathrm{C}_{20} \mathrm{H}_{29} \mathrm{O}^{+}\left[M-\mathrm{H}_{2} \mathrm{O}+\mathrm{H}\right]^{+}:$: 285.2213, found: 285.2213;

IR (neat sample): 3444, 2957, 1651, 1454, 1376, 1038, $889 \mathrm{~cm}^{-1}$;

$[\alpha]_{D}{ }^{4}:+253.5\left(c 0.45, \mathrm{CHCl}_{3}\right)$.

7b: (containing some inseparable E-geometric isomer on sidechain)

$\boldsymbol{R}_{\mathrm{f}}=0.44(\mathrm{PE} / \mathrm{EA}=5: 1, \mathrm{CAM})$;

${ }^{1} \mathrm{H}$ NMR $\left(400 \mathrm{MHz}, \mathrm{CDCl}_{3}\right) \delta 6.21(\mathrm{~d}, J=2.9 \mathrm{~Hz}, 1 \mathrm{H}), 5.77(\mathrm{~d}, J=11.8 \mathrm{~Hz}, 1 \mathrm{H}), 5.22$ (dd, J = 11.8, $10.2 \mathrm{~Hz}, 1 \mathrm{H}), 4.92-4.79(\mathrm{~m}, 1 \mathrm{H}), 4.15(\mathrm{td}, J=8.2,5.7 \mathrm{~Hz}, 1 \mathrm{H}), 3.37(\mathrm{~d}, J=8.3 \mathrm{~Hz}, 1 \mathrm{H}), 2.89$ (dqd, $J=10.1,6.8,0.9 \mathrm{~Hz}, 1 \mathrm{H}$ ), 2.57 (qdd, $J=7.3,4.2,3.0 \mathrm{~Hz}, 1 \mathrm{H}$ ), $1.91-1.83(\mathrm{~m}, 4 \mathrm{H}), 1.77$ $1.73(\mathrm{~m}, 1 \mathrm{H}), 1.58$ (ddd, J = 13.7, 8.0, 7.0 Hz, 1H), 1.42 (ddd, J = 13.7, 6.9, $5.6 \mathrm{~Hz}, 1 \mathrm{H}$ ), 1.32 (d, $J=7.3 \mathrm{~Hz}, 3 \mathrm{H}), 1.28(\mathrm{dd}, J=8.4,2.8 \mathrm{~Hz}, 1 \mathrm{H}), 1.15(\mathrm{~s}, 3 \mathrm{H}), 1.13-1.09(\mathrm{~m}, 1 \mathrm{H}), 1.02(\mathrm{~s}, 3 \mathrm{H}), 1.00$ (d, $J=6.6 \mathrm{~Hz}, 3 \mathrm{H}) \mathrm{ppm}$;

${ }^{13} \mathrm{C}$ NMR $\left(100 \mathrm{MHz}, \mathrm{CDCl}_{3}\right) \delta$ 211.9, 142.8, 141.7, 137.9, 137.7, 129.7, 115.4, 74.3, 47.1, 44.8, 29.7, 28.1, 27.5, 26.0, 24.8, 23.6, 23.4, 21.1, 16.0, 15.8 ppm;

HRMS (ESI): $\mathrm{m} / \mathrm{z}$ calcd for $\mathrm{C}_{20} \mathrm{H}_{29} \mathrm{O}^{+}\left[\mathrm{M}-\mathrm{H}_{2} \mathrm{O}+\mathrm{H}\right]^{+}:$:285.2213, found: 285.2213 .

IR (neat sample): 3473, 2928, 1638, 1454, 1375, 1054, 982, 890, $778 \mathrm{~cm}^{-1}$;

$[\alpha]_{D}^{3:}+200.4\left(c 1.45, \mathrm{CHCl}_{3}\right)$.

7c: (containing some inseparable E-geometric isomer on sidechain)

$\boldsymbol{R}_{\mathrm{f}}=0.40(\mathrm{PE} / \mathrm{EA}=5: 1, \mathrm{CAM})$;

${ }^{1} \mathrm{H}$ NMR $\left(400 \mathrm{MHz}, \mathrm{CDCl}_{3}\right) \delta 6.26(\mathrm{~d}, J=2.9 \mathrm{~Hz}, 1 \mathrm{H}), 5.77(\mathrm{~d}, J=11.7 \mathrm{~Hz}, 1 \mathrm{H}), 5.22$ (dd, J = 11.8, $10.2 \mathrm{~Hz}, 1 \mathrm{H}), 4.93-4.72(\mathrm{~m}, 2 \mathrm{H}), 4.16(\mathrm{td}, J=7.8,5.7 \mathrm{~Hz}, 1 \mathrm{H}), 3.32(\mathrm{~d}, J=8.3 \mathrm{~Hz}, 1 \mathrm{H}), 2.94-$ $2.81(\mathrm{~m}, 1 \mathrm{H}), 2.71(\mathrm{dqd}, J=11.6,7.1,2.5 \mathrm{~Hz}, 1 \mathrm{H}), 1.89-1.81(\mathrm{~m}, 4 \mathrm{H}), 1.63-1.56(\mathrm{~m}, 1 \mathrm{H}), 1.48$ $-1.39(\mathrm{~m}, 2 \mathrm{H}), 1.26(\mathrm{dd}, J=8.6,2.8 \mathrm{~Hz}, 1 \mathrm{H}), 1.24-1.18(\mathrm{~m}, 1 \mathrm{H}), 1.12(\mathrm{~s}, 3 \mathrm{H}), 1.09(\mathrm{~d}, J=7.1$ $\mathrm{Hz}, 3 \mathrm{H}), 1.02-0.99(\mathrm{~m}, 6 \mathrm{H}) \mathrm{ppm}$;

${ }^{13} \mathrm{C}$ NMR $\left(100 \mathrm{MHz}, \mathrm{CDCl}_{3}\right) \delta 211.4,143.1,141.8,138.7,137.7,129.8,115.3,74.3,47.2,45.1$, 29.7, 29.5, 29.4, 28.0, 27.5, 23.9, 23.6, 21.1, 18.0, 15.9 ppm;

HRMS (ESI): $\mathrm{m} / \mathrm{z}$ calcd for $\mathrm{C}_{20} \mathrm{H}_{29} \mathrm{O}^{+}\left[M-\mathrm{H}_{2} \mathrm{O}+\mathrm{H}\right]^{+}:$: 285.2213, found: 285.2213;

IR (neat sample): 3459, 2959, 1651, 1453, 1375, 1052, 969, 885, $780 \mathrm{~cm}^{-1}$; 
$[\alpha]_{D}^{3:}+299.1\left(c 0.7, \mathrm{CHCl}_{3}\right)$.

\section{Synthesis of 6}

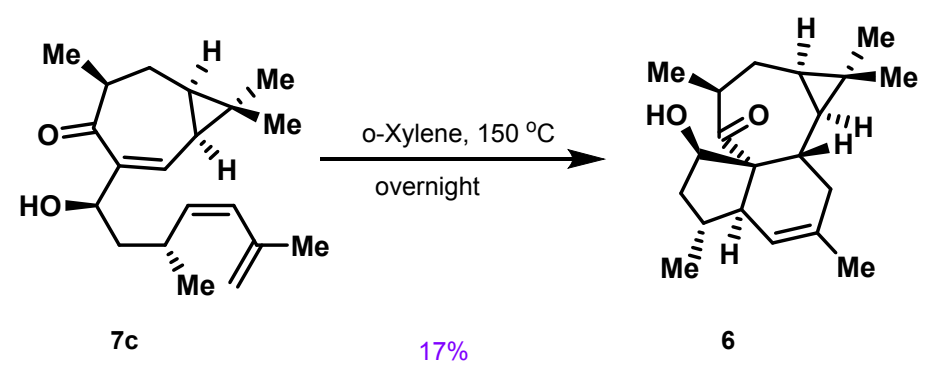

Triene $7 \mathrm{c}(56.0 \mathrm{mg}, 0.200 \mathrm{mmol})$ was dissolved in o-xylene $(4.00 \mathrm{~mL}, 0.050 \mathrm{M})$ in a sealed tube, and the solution was degassed with $\mathrm{N}_{2}$ via ultrasonic for 10 min. Afterwards, the reaction was allowed to warm to $150^{\circ} \mathrm{C}$ and stirred at this temperature overnight. Then the solvent was removed under reduced pressure, and the crude product was purified by flash column chromatography on silica gel $(P E / E A=10 / 1)$ to give product $6(10.0 \mathrm{mg}, 17 \%)$ as a colourless crystalline solid.

$\mathbf{R}_{\mathbf{f}}=0.37(\mathrm{PE} / \mathrm{EA}=6 / 1, \mathrm{CAM})$;

${ }^{1} \mathrm{H}$ NMR $\left(500 \mathrm{MHz}, \mathrm{CDCl}_{3}\right) \delta 5.49$ (ddd, $\left.J=4.4,2.7,1.4 \mathrm{~Hz}, 1 \mathrm{H}\right), 4.42(\mathrm{~d}, J=5.5 \mathrm{~Hz}, 1 \mathrm{H}), 2.99$ (dt, $J=12.1,7.2 \mathrm{~Hz}, 2 \mathrm{H}$ ), 2.54 (ddtd, $J=16.6,5.0,2.5,1.2 \mathrm{~Hz}, 1 \mathrm{H}$ ), 2.46 (ddd, $J=11.2,5.9,1.7$ $\mathrm{Hz}, 1 \mathrm{H}$ ), 1.85 (dddd, $J=14.2,12.2,7.0,4.9 \mathrm{~Hz}, 2 \mathrm{H}), 1.66(\mathrm{~d}, J=1.4 \mathrm{~Hz}, 3 \mathrm{H}), 1.65-1.59(\mathrm{~m}, 3 \mathrm{H})$, $1.53-1.41(\mathrm{~m}, 2 \mathrm{H}), 1.29(\mathrm{~d}, J=7.5 \mathrm{~Hz}, 3 \mathrm{H}), 1.09(\mathrm{~s}, 3 \mathrm{H}), 1.07(\mathrm{~d}, J=6.9 \mathrm{~Hz}, 3 \mathrm{H}), 0.94(\mathrm{~s}, 3 \mathrm{H})$, $0.73-0.61(\mathrm{~m}, 2 \mathrm{H}) \mathrm{ppm}$;

${ }^{13} \mathrm{C}$ NMR $\left(125 \mathrm{MHz}, \mathrm{CDCl}_{3}\right) \delta 216.7,129.3,125.8,78.3,66.6,52.4,44.9,42.0,39.6,35.1,30.8$, 29.6, 29.1, 29.1, 25.8, 24.4, 19.8, 19.5, 18.5, 14.7 ppm;

HRMS (ESI): $\mathrm{m} / \mathrm{z}$ calcd for $\mathrm{C}_{20} \mathrm{H}_{29} \mathrm{O}^{+}\left[\mathrm{M}-\mathrm{H}_{2} \mathrm{O}+\mathrm{H}\right]^{+}: 285.2213$, found: 285.2213;

IR (neat sample): 3443, 2926, 2872, 1662, 1457, 1375, 1085, 1038, 996, 960, 947, 828, 803, 733 $\mathrm{cm}^{-1}$.

$[\alpha]_{D}^{4}:-52.4\left(c \quad 0.95, \mathrm{CHCl}_{3}\right)$. 


\section{Synthesis of SI-3}

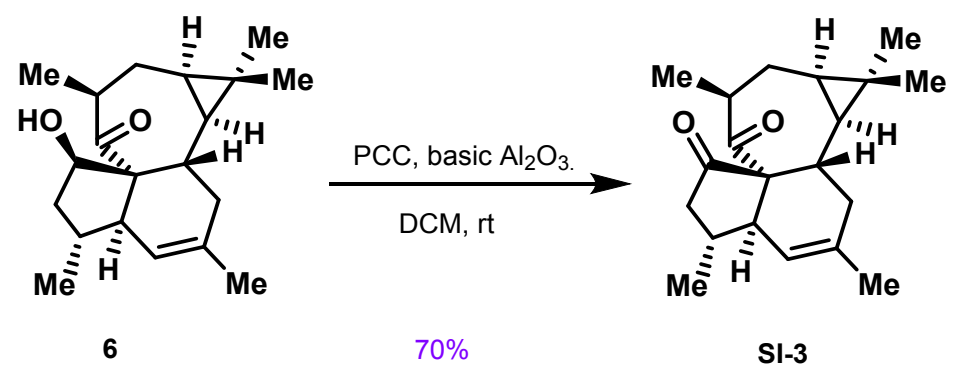

To the stirring solution of alcohol $6\left(10.0 \mathrm{mg}, 33.0 \mu \mathrm{mol}, 1.00\right.$ equiv) in $\mathrm{CH}_{2} \mathrm{Cl}_{2}(0.660 \mathrm{~mL}, 0.050$ M) were added basic $\mathrm{Al}_{2} \mathrm{O}_{3}(57.0 \mathrm{mg})$ and pyridinium chlorochromate (14.2 $\mathrm{mg}, 2.00$ equiv) successively at room temperature. The reaction was stirred for $1 \mathrm{~h}$ before it was filtered through a short pad of silica gel and washed with $\mathrm{CH}_{2} \mathrm{Cl}_{2}$ three times. The resultant filtrate was concentrated under reduced pressure. The obtained solid was purified by column chromatography on silica gel (PE/EA = 15/1), yielding diketone SI-3 $(7.00 \mathrm{mg}, 70 \%)$ as a colourless crystalline solid.

$\mathbf{R}_{\mathbf{f}}=0.59(\mathrm{PE} / \mathrm{EA}=6 / 1, \mathrm{CAM})$;

${ }^{1} \mathrm{H}$ NMR $\left(500 \mathrm{MHz}, \mathrm{CDCl}_{3}\right) \delta 5.36(\mathrm{dp}, J=2.7,1.3 \mathrm{~Hz}, 1 \mathrm{H}), 3.10-3.01(\mathrm{~m}, 1 \mathrm{H}), 2.99(\mathrm{~s}, 1 \mathrm{H}), 2.34$ (dd, $J=19.1,9.0 \mathrm{~Hz}, 1 \mathrm{H}), 2.30-2.23(\mathrm{~m}, 1 \mathrm{H}), 2.20-2.08(\mathrm{~m}, 1 \mathrm{H}), 1.96-1.87(\mathrm{~m}, 2 \mathrm{H}), 1.82$ (ddd, $J=14.5,6.5,2.2 \mathrm{~Hz}, 1 \mathrm{H}), 1.68-1.56(\mathrm{~m}, 5 \mathrm{H}), 1.06(\mathrm{~d}, J=6.7 \mathrm{~Hz}, 3 \mathrm{H}), 1.05-1.00(\mathrm{~m}, 6 \mathrm{H})$, 0.96 (s, 3H), $0.88-0.81(\mathrm{~m}, 1 \mathrm{H}), 0.69$ (dd, J = 10.4, $9.4 \mathrm{~Hz}, 1 \mathrm{H}) \mathrm{ppm}$;

${ }^{13} \mathrm{C}$ NMR $\left(125 \mathrm{MHz}, \mathrm{CDCl}_{3}\right) \delta 214.9,214.8,133.7,124.5,70.6,45.9,45.7,41.8,34.8,34.8,30.2$, $30.1,29.3,28.5,27.1,24.4,21.3,18.2,17.5,15.3$ ppm;

HRMS (ESI): $\mathrm{m} / \mathrm{z}$ calcd for $\mathrm{C}_{20} \mathrm{H}_{29} \mathrm{O}_{2}{ }^{+}[M+\mathrm{H}]^{+}:$301.2162, found: 301.2159 ;

IR (neat sample): 2963, 2930, 2891, 1743, 1672, 1457, 1376, 1195, 1115, 1047, 1029, 968, 953, $916,834,805,738 \mathrm{~cm}^{-1}$;

$[\alpha]_{\mathrm{D}}^{4:}+114.7$ (c 0.5, $\left.\mathrm{CHCl}_{3}\right)$. 


\section{Synthesis of 17c}

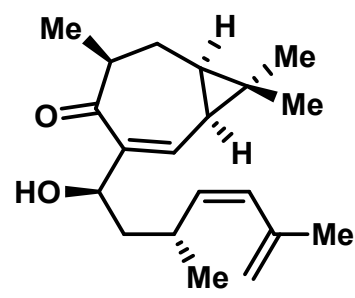

7c

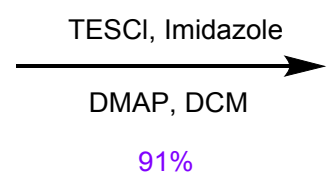

$91 \%$

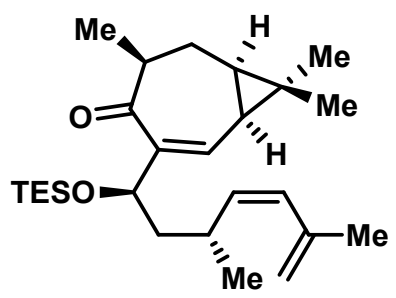

$17 \mathrm{c}$

To a stirring solution of $7 \mathrm{c}\left(115 \mathrm{mg}, 0.380 \mathrm{mmol}, 1.00\right.$ equiv) in $\mathrm{CH}_{2} \mathrm{Cl}_{2}(4.00 \mathrm{~mL}, 0.10 \mathrm{M})$ were added imidazole (103 mg, $1.52 \mathrm{mmol}, 4.00$ equiv), 4-dimethylaminopyridine (4.60 mg, 0.0380 mmol, 0.100 equiv) and chlorotriethylsilane (130 $\mu \mathrm{L}, 0.760 \mathrm{mmol}, 2.00$ equiv) at $0{ }^{\circ} \mathrm{C}$ successively. Then the reaction was allowed to warm to room temperature and stirred for 30 min at this temperature. The saturated aq. $\mathrm{NH}_{4} \mathrm{Cl}$ was added to quench the reaction, and the mixture was extracted with $\mathrm{CH}_{2} \mathrm{Cl}_{2}(3 \times 10 \mathrm{~mL})$. The combined organic layers were dried over $\mathrm{MgSO}_{4}$, filtered and concentrated in vacuo. The resultant residue was purified by column chromatography on silica gel $(\mathrm{PE} / \mathrm{EA}=40 / 1)$, yielding $17 \mathrm{c}(144 \mathrm{mg}, 91 \%)$ as a colourless oil.

$\mathbf{R}_{\mathrm{f}}=0.51(\mathrm{PE} / \mathrm{EA}=20 / 1, \mathrm{CAM})$;

${ }^{1} \mathrm{H}$ NMR (400 MHz, $\left.\mathrm{CDCl}_{3}\right) \delta 6.56(\mathrm{dt}, J=3.1,1.0 \mathrm{~Hz}, 1 \mathrm{H}), 5.68(\mathrm{dt}, J=11.8,1.0 \mathrm{~Hz}, 1 \mathrm{H}), 5.14$ (dd, $J=11.8,10.2 \mathrm{~Hz}, 1 \mathrm{H}), 4.92-4.84(\mathrm{~m}, 1 \mathrm{H}), 4.66(\mathrm{ddd}, J=7.1,4.0,1.1 \mathrm{~Hz}, 1 \mathrm{H}), 3.03-2.86$ (m, 1H), $2.77-2.64(\mathrm{~m}, 1 \mathrm{H}), 1.91-1.85(\mathrm{~m}, 4 \mathrm{H}), 1.49-1.37(\mathrm{~m}, 3 \mathrm{H}), 1.27(\mathrm{ddt}, J=4.6,3.5,1.9$ $\mathrm{Hz}, 2 \mathrm{H}), 1.15(\mathrm{~s}, 3 \mathrm{H}), 1.08(\mathrm{~d}, J=7.0 \mathrm{~Hz}, 3 \mathrm{H}), 1.01(\mathrm{~d}, J=6.6 \mathrm{~Hz}, 3 \mathrm{H}), 0.97(\mathrm{~s}, 3 \mathrm{H}), 0.93(\mathrm{t}, J=$ $7.9 \mathrm{~Hz}, 9 \mathrm{H}$ ), 0.57 (q, $J=8.0 \mathrm{~Hz} 6 \mathrm{H}$ ) ppm;

${ }^{13} \mathrm{C}$ NMR $\left(100 \mathrm{MHz}, \mathrm{CDCl}_{3}\right) \delta 206.2,144.5,142.1,138.7,137.8,128.4,115.3,69.1,47.3,47.2$, $30.3,29.8,28.9,28.5,28.3,24.9,23.5,20.7,17.6,16.2,7.1,5.1$ ppm;

HRMS (ESI): $\mathrm{m} / \mathrm{z}$ calcd for $\mathrm{C}_{26} \mathrm{H}_{44} \mathrm{O}_{2} \mathrm{SiNa}^{+}[\mathrm{M}+\mathrm{Na}]^{+}:$: 439.3003, found: 439.3006;

IR (neat sample): 2957, 2876, 1655, 1456, 1375, 1239, 1086, 1006, 971, 942, 886, $739 \mathrm{~cm}^{-1}$; $[\alpha]_{D}^{4}:+154.1\left(c 0.35, \mathrm{CHCl}_{3}\right)$. 


\section{Synthesis of 19}

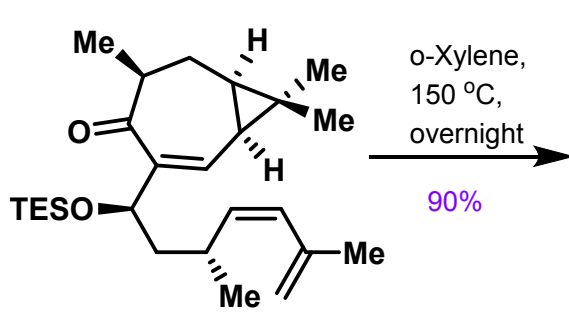

$17 c$

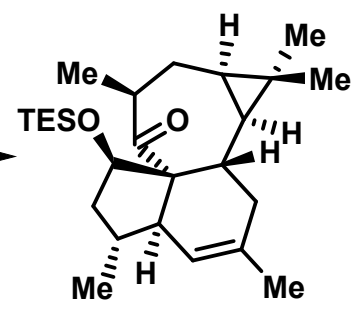

19

TES-protected triene 17c (133 mg, $0.320 \mathrm{mmol})$ was dissolved in o-xylene $(6.40 \mathrm{~mL}, 0.050 \mathrm{M})$ in a sealed tube, and the solution was degassed with $\mathrm{N}_{2}$ via ultrasonic for $10 \mathrm{~min}$. Afterwards, the reaction was allowed to warm to $150^{\circ} \mathrm{C}$ and stirred at this temperature overnight. Then the solvent was removed under reduced pressure, and the crude product was purified by flash column chromatography on silica gel $(P E / E A=40 / 1)$ to give product $19(120 \mathrm{mg}, 90.2 \%)$ as a colourless oil.

$\mathbf{R}_{\mathrm{f}}=0.52(\mathrm{PE} / \mathrm{EA}=20 / 1, \mathrm{CAM})$;

${ }^{1} \mathrm{H}$ NMR $\left(500 \mathrm{MHz}, \mathrm{CDCl}_{3}\right) \delta 5.47$ (ddd, $\left.J=5.4,2.9,1.5 \mathrm{~Hz}, 1 \mathrm{H}\right), 4.37$ (t, $\left.J=4.9 \mathrm{~Hz}, 1 \mathrm{H}\right), 2.97$ (d, $J=5.5 \mathrm{~Hz}, 1 \mathrm{H}), 2.95-2.87(\mathrm{~m}, 1 \mathrm{H}), 2.59-2.53(\mathrm{~m}, 1 \mathrm{H}), 2.42(\mathrm{ddd}, J=11.2,5.3,2.0 \mathrm{~Hz}, 1 \mathrm{H})$, $1.85(\mathrm{dq}, J=13.5,6.8 \mathrm{~Hz}, 1 \mathrm{H}), 1.80-1.73(\mathrm{~m}, 1 \mathrm{H}), 1.65(\mathrm{~d}, J=1.4 \mathrm{~Hz}, 3 \mathrm{H}), 1.56-1.45(\mathrm{~m}, 3 \mathrm{H})$, 1.33 (ddd, $J=12.5,7.8,5.0 \mathrm{~Hz}, 1 \mathrm{H}$ ), 1.27 (d, $J=7.5 \mathrm{~Hz}, 3 \mathrm{H}$ ), 1.07 (s, 3H), 1.01 (d, $J=6.9 \mathrm{~Hz}$, $3 \mathrm{H}), 0.96(\mathrm{t}, J=7.9 \mathrm{~Hz}, 9 \mathrm{H}), 0.92(\mathrm{~s}, 3 \mathrm{H}), 0.66-0.57(\mathrm{~m}, 8 \mathrm{H}) \mathrm{ppm}$;

${ }^{13} \mathrm{C}$ NMR $\left(125 \mathrm{MHz}, \mathrm{CDCl}_{3}\right) \delta$ 216.7, 130.3, 124.7, 77.8, 67.3, 52.7, 45.2, 42.4, 40.0, 34.8, 31.5, 29.1, 28.9, 28.5, 26.2, 24.6, 20.3, 19.3, 18.4, 14.6, 7.0, 5.3 ppm;

HRMS (ESI): $\mathrm{m} / \mathrm{z}$ calcd for $\mathrm{C}_{26} \mathrm{H}_{45} \mathrm{O}_{2} \mathrm{Si}^{+}[\mathrm{M}+\mathrm{H}]^{+}:$:17.3183, found: 417.3184;

IR (neat sample): 2954, 2875, 1678, 1457, 1413, 1376, 1237, 1103, 1050, 1003, 973, 902, 821, $720 \mathrm{~cm}^{-1}$;

$[\alpha]_{\mathrm{D}}{ }^{3}:-77.4\left(c\right.$ 1.15, $\left.\mathrm{CHCl}_{3}\right)$. 


\section{Synthesis of $17 \mathrm{~b}$ and 19}

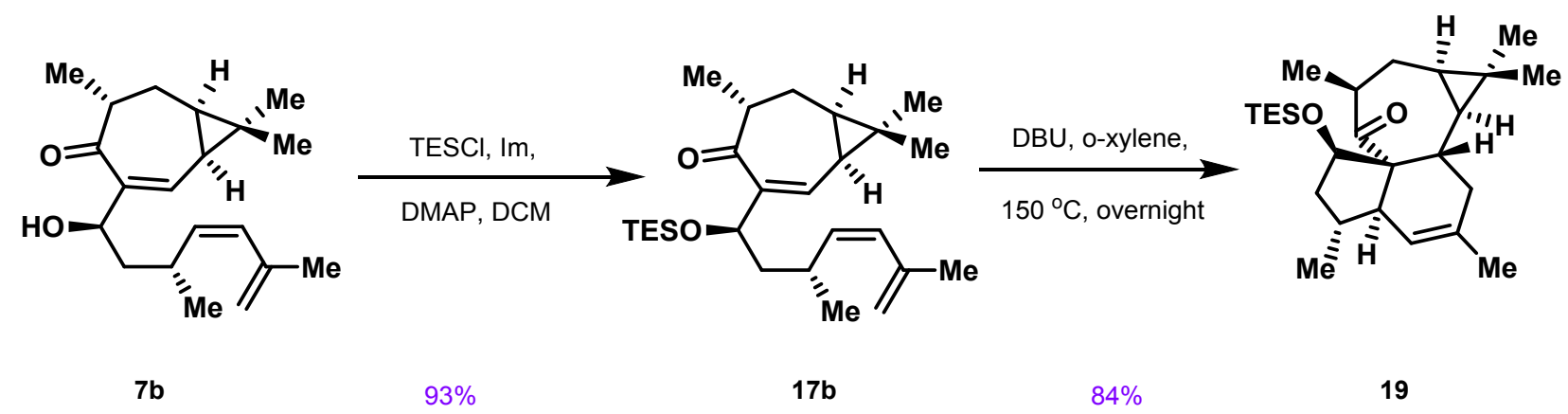

To a stirring solution of $7 \mathbf{b}$ (29.0 mg, $96.0 \mu \mathrm{mol}, 1.00$ equiv) in $\mathrm{CH}_{2} \mathrm{Cl}_{2}$ (2.00 mL, $\left.0.050 \mathrm{M}\right)$ were added imidazole (20 mg, $0.29 \mathrm{mmol}, 3.0$ equiv), 4-dimethylaminopyridine (1.20 mg, $9.60 \mu \mathrm{mol}$, 0.100 equiv) and chlorotriethylsilane (24.0 $\mu \mathrm{L}, 0.144 \mathrm{mmol}, 1.50$ equiv) at $0{ }^{\circ} \mathrm{C}$ successively. Then the reaction was allowed to warm to room temperature and stirred for $30 \mathrm{~min}$ at this temperature. Saturated aq. $\mathrm{NH}_{4} \mathrm{Cl}$ was added to quench the reaction, and the mixture was extracted with $\mathrm{CH}_{2} \mathrm{Cl}_{2}$. The combined organic layers were dried over $\mathrm{MgSO}_{4}$, filtered and concentrated in vacuo. The resultant residue was purified by column chromatography on silica gel (PE/EA $=40 / 1)$, yielding $17 \mathrm{~b}$ (37.0 $\mathrm{mg}, 93 \%)$ as a colourless oil.

$\mathbf{R}_{\mathrm{f}}=0.44$ (PE/EA = 20/1, CAM);

${ }^{1} \mathrm{H}$ NMR $\left(400 \mathrm{MHz}, \mathrm{CDCl}_{3}\right) \delta 6.51(\mathrm{~d}, J=3.5 \mathrm{~Hz}, 1 \mathrm{H}), 5.69$ (d, J=11.8 Hz, 1H), 5.16 (dd, J = 11.8, $10.2 \mathrm{~Hz}, 1 \mathrm{H}), 4.90-4.88(\mathrm{~m}, 2 \mathrm{H}), 4.57$ (dd, $J=7.9,3.4 \mathrm{~Hz}, 1 \mathrm{H}), 3.04-2.89(\mathrm{~m}, 1 \mathrm{H}), 2.63$ (qdd, $J=7.3,4.8,2.5 \mathrm{~Hz}, 1 \mathrm{H}), 1.95-1.86(\mathrm{~m}, 4 \mathrm{H}), 1.70$ (ddd, $J=14.9,10.1,2.5 \mathrm{~Hz}, 1 \mathrm{H}), 1.49-1.36$ (m, 2H), $1.32-1.25(\mathrm{~m}, 4 \mathrm{H}), 1.17(\mathrm{~s}, 3 \mathrm{H}), 1.13-1.07(\mathrm{~m}, 1 \mathrm{H}), 1.01(\mathrm{~d}, \mathrm{~J}=6.6 \mathrm{~Hz}, 3 \mathrm{H}), 0.99(\mathrm{~s}$, $3 \mathrm{H}), 0.91(\mathrm{t}, J=7.9 \mathrm{~Hz}, 9 \mathrm{H}), 0.54(\mathrm{q}, J=8.0 \mathrm{~Hz}, 6 \mathrm{H}) \mathrm{ppm}$;

${ }^{13} \mathrm{C}$ NMR $\left(100 \mathrm{MHz}, \mathrm{CDCl}_{3}\right) \delta 207.9,144.1,142.1,138.6,137.3,128.4,115.3,68.8,47.4,47.2$, $28.9,28.7,28.3,26.3,24.6,23.9,23.5,20.5,16.1,16.1,7.1,5.0$ ppm;

HRMS (ESI): $\mathrm{m} / \mathrm{z}$ calcd for $\mathrm{C}_{26} \mathrm{H}_{44} \mathrm{O}_{2} \mathrm{SiNa}^{+}[M+\mathrm{Na}]^{+}: 439.3003$, found: 439.3005;

IR (neat sample): 2956, 2876, 1648, 1456, 1414, 1376, 1237, 1087, 1003, 964, 944, 883, 843, $724 \mathrm{~cm}^{-1}$;

$[\alpha]_{\mathrm{D}}^{4}:+200.7\left(c 0.8, \mathrm{CHCl}_{3}\right)$.

TES-protected triene 17b (10.0 mg, $24.0 \mu \mathrm{mol})$ and 1,8-Diazabicyclo[5.4.0]undec-7-ene (DBU, $4.30 \mu \mathrm{L}, 29.0 \mu \mathrm{mol}, 1.20$ equiv) were dissolved in o-xylene $(1.00 \mathrm{~mL}, 0.020 \mathrm{M})$ in a sealed tube 
and the solution was degassed with $\mathrm{N}_{2}$ via ultrasonic for 10 min. Afterwards, the reaction was allowed to warm to $150^{\circ} \mathrm{C}$ and stirred at this temperature overnight. Then the solvent was removed directly under reduced pressure, and the crude product was purified by flash column chromatography on silica gel $(P E / E A=40 / 1)$ to give product $19(8.00 \mathrm{mg}, 84 \%)$ as a colourless oil.

$\mathbf{R}_{\mathbf{f}}=0.52$ (PE/EA = 20/1, CAM);

${ }^{1} \mathrm{H}$ NMR $\left(500 \mathrm{MHz}, \mathrm{CDCl}_{3}\right) \delta 5.47$ (ddd, $\left.J=5.4,2.9,1.5 \mathrm{~Hz}, 1 \mathrm{H}\right), 4.37$ (t, $\left.J=4.9 \mathrm{~Hz}, 1 \mathrm{H}\right), 2.97$ (d, $J=5.5 \mathrm{~Hz}, 1 \mathrm{H}), 2.95-2.87(\mathrm{~m}, 1 \mathrm{H}), 2.59-2.53(\mathrm{~m}, 1 \mathrm{H}), 2.42(\mathrm{ddd}, J=11.2,5.3,2.0 \mathrm{~Hz}, 1 \mathrm{H})$, $1.85(\mathrm{dq}, J=13.5,6.8 \mathrm{~Hz}, 1 \mathrm{H}), 1.80-1.73(\mathrm{~m}, 1 \mathrm{H}), 1.65(\mathrm{~d}, J=1.4 \mathrm{~Hz}, 3 \mathrm{H}), 1.56-1.45(\mathrm{~m}, 3 \mathrm{H})$, 1.33 (ddd, $J=12.5,7.8,5.0 \mathrm{~Hz}, 1 \mathrm{H}$ ), 1.27 (d, $J=7.5 \mathrm{~Hz}, 3 \mathrm{H}$ ), 1.07 (s, 3H), 1.01 (d, $J=6.9 \mathrm{~Hz}$, $3 \mathrm{H}), 0.96(\mathrm{t}, J=7.9 \mathrm{~Hz}, 9 \mathrm{H}), 0.92(\mathrm{~s}, 3 \mathrm{H}), 0.66-0.57(\mathrm{~m}, 8 \mathrm{H}) \mathrm{ppm}$;

${ }^{13} \mathrm{C}$ NMR $\left(125 \mathrm{MHz}, \mathrm{CDCl}_{3}\right) \delta 216.7,130.3,124.7,77.8,67.3,52.7,45.2,42.4,40.0,34.8,31.5$, 29.1, 28.9, 28.5, 26.2, 24.6, 20.3, 19.3, 18.4, 14.6, 7.0, 5.3 ppm;

HRMS (ESI): $\mathrm{m} / \mathrm{z}$ calcd for $\mathrm{C}_{26} \mathrm{H}_{45} \mathrm{O}_{2} \mathrm{Si}^{+}[M+\mathrm{H}]^{+}:$: 17.3183 , found: 417.3184;

IR (neat sample): 2954, 2875, 1678, 1457, 1413, 1376, 1237, 1103, 1050, 1003, 973, 902, 821, $720 \mathrm{~cm}^{-1}$;

$[\alpha]_{\mathrm{D}}^{3}:-77.4\left(c 1.15, \mathrm{CHCl}_{3}\right)$.

\section{Synthesis of $17 a$ and 18}

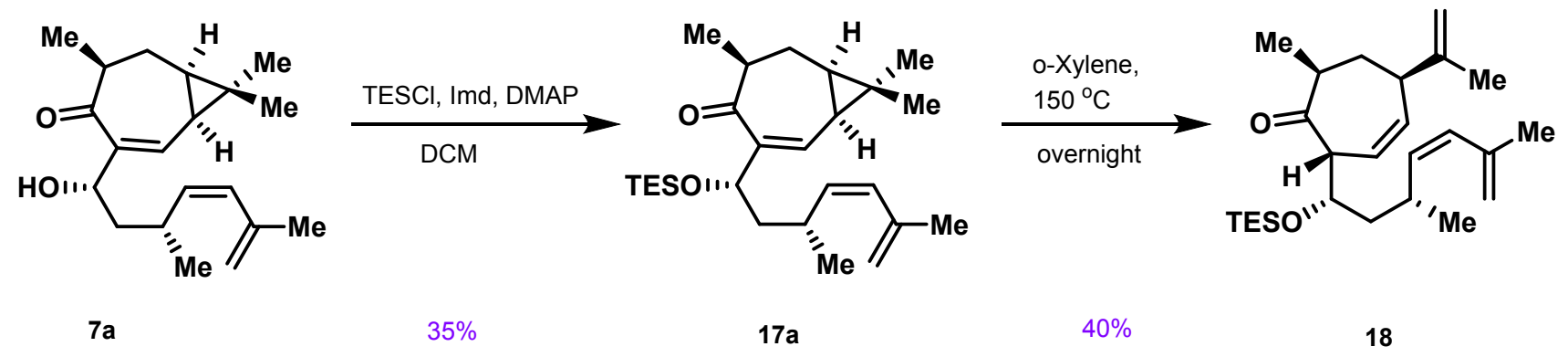

To a stirring solution of $7 \mathrm{a}$ with inseparable impurities (38.0 mg, $0.125 \mathrm{mmol}, 1.00$ equiv) in $\mathrm{CH}_{2} \mathrm{Cl}_{2}$ (1.25 mL, $0.10 \mathrm{M}$ ) were added imidazole (34.2 mg, $0.500 \mathrm{mmol}, 4.00$ equiv), 4dimethylaminopyridine $(1.50 \mathrm{mg}, 0.0125 \mathrm{mmol}, 0.100$ equiv) and chlorotriethylsilane $(42.0 \mu \mathrm{L}$, $0.250 \mathrm{mmol}, 2.00$ equiv) at $0{ }^{\circ} \mathrm{C}$ successively. Then the reaction was allowed to warm to room temperature and stirred for $30 \mathrm{~min}$ at this temperature. Then the saturated aq. $\mathrm{NH}_{4} \mathrm{Cl}$ was added to quench the reaction, and the mixture was extracted with $\mathrm{CH}_{2} \mathrm{Cl}_{2}(\times 3)$. The combined organic 
layers were dried over $\mathrm{MgSO}_{4}$, filtered and concentrated in vacuo. The resultant residue was purified by column chromatography on silica gel $(P E / E A=60 / 1)$, yielding $17 a(18.0 \mathrm{mg}, 35 \%)$ as a colourless oil.

$\mathbf{R}_{\mathrm{f}}=0.48$ (PE/EA = 20/1, CAM);

${ }^{1} \mathrm{H}$ NMR (400 MHz, $\left.\mathrm{CDCl}_{3}\right) \delta 6.55$ (dt, $\left.J=3.1,1.0 \mathrm{~Hz}, 1 \mathrm{H}\right), 5.74(\mathrm{dt}, J=11.8,1.1 \mathrm{~Hz}, 1 \mathrm{H}), 5.29$ (dd, $J=11.9,9.8 \mathrm{~Hz}, 1 \mathrm{H}), 4.93-4.79(\mathrm{~m}, 2 \mathrm{H}), 4.61(\mathrm{t}, J=6.1 \mathrm{~Hz}, 1 \mathrm{H}), 2.80-2.65(\mathrm{~m}, 2 \mathrm{H}), 1.94$ $-1.83(\mathrm{~m}, 4 \mathrm{H}), 1.53-1.38(\mathrm{~m}, 3 \mathrm{H}), 1.27$ (ddd, $J=6.3,5.1,2.1 \mathrm{~Hz}, 2 \mathrm{H}), 1.15(\mathrm{~s}, 3 \mathrm{H}), 1.08(\mathrm{~d}, J=$ $7.0 \mathrm{~Hz}, 3 \mathrm{H}$ ), 0.97 (d, J = 7.3 Hz, 6H), 0.90 (t, J=8.0 Hz, 9H), 0.53 (q, J = 7.8 Hz, 6H) ppm;

${ }^{13} \mathrm{C}$ NMR $\left(100 \mathrm{MHz}, \mathrm{CDCl}_{3}\right) \delta$ 206.2, 144.8, 142.0, 138.6, 137.9, 129.0, 114.9, 69.71, 48.4, 47.3, $30.2,29.9,29.5,28.5,28.3,24.9,23.6,22.3,17.7,16.2,7.1,5.0$ ppm;

HRMS (ESI): $\mathrm{m} / \mathrm{z}$ calcd for $\mathrm{C}_{26} \mathrm{H}_{44} \mathrm{O}_{2} \mathrm{SiNa}^{+}[M+\mathrm{Na}]^{+}:$439.3003, found: 439.3005;

IR (neat sample): 2954, 2875, 1655, 1455, 1414, 1375, 1238, 1199, 1060, 1005, 970, 941, 885, $726 \mathrm{~cm}^{-1}$;

$[\alpha]_{D}^{3:}+116.6\left(c 1.7, \mathrm{CHCl}_{3}\right)$.

TES-protected triene $17 \mathrm{a}(10.0 \mathrm{mg}, 24.0 \mu \mathrm{mol})$ was dissolved in o-xylene $(2.40 \mathrm{~mL}, 0.010 \mathrm{M})$ in a sealed tube, and the solution was degassed with $\mathrm{N}_{2}$ via ultrasonic for $10 \mathrm{~min}$. Afterwards, the reaction was allowed to warm to $150{ }^{\circ} \mathrm{C}$ and stirred at this temperature overnight. Then the solvent was removed directly under reduced pressure, and the crude product was purified by flash column chromatography on silica gel $(P E / E A=60 / 1)$ to give vinyl cyclopropane rearrangement product $18(4.00 \mathrm{mg}, 40 \%)$ as a slightly yellow oil.

$\mathbf{R}_{\mathrm{f}}=0.42(\mathrm{PE} / \mathrm{EA}=20 / 1, \mathrm{CAM})$;

${ }^{1} \mathrm{H}$ NMR $\left(600 \mathrm{MHz}, \mathrm{CDCl}_{3}\right) \delta 5.79(\mathrm{~d}, J=11.9 \mathrm{~Hz}, 1 \mathrm{H}), 5.57-5.54(\mathrm{~m}, 2 \mathrm{H}), 5.45$ (dt, J = 11.7, 2.8 $\mathrm{Hz}, 1 \mathrm{H}), 5.29$ (dd, $J=11.8,10.0 \mathrm{~Hz}, 1 \mathrm{H}), 4.98-4.90(\mathrm{~m}, 2 \mathrm{H}), 4.77$ (dd, $J=4.4,2.8 \mathrm{~Hz}, 2 \mathrm{H}), 4.09$ $(\mathrm{td}, J=7.0,2.7 \mathrm{~Hz}, 1 \mathrm{H}), 3.76(\mathrm{dp}, J=6.4,3.1 \mathrm{~Hz}, 1 \mathrm{H}), 2.99-2.89(\mathrm{~m}, 2 \mathrm{H}), 2.53(\mathrm{dtt}, J=14.3$, 7.1, 3.6 Hz, 1H), $1.90(\mathrm{~d}, J=1.3 \mathrm{~Hz}, 3 \mathrm{H}), 1.89-1.85(\mathrm{~m}, 1 \mathrm{H}), 1.73(\mathrm{~d}, J=1.1 \mathrm{~Hz}, 3 \mathrm{H}), 1.68-$ $1.62(\mathrm{~m}, 1 \mathrm{H}), 1.52$ (ddd, $J=11.9,8.3,3.3 \mathrm{~Hz}, 2 \mathrm{H}), 1.12(\mathrm{~d}, J=7.1 \mathrm{~Hz}, 4 \mathrm{H}), 1.03(\mathrm{~d}, J=6.7 \mathrm{~Hz}$, $3 \mathrm{H}), 0.93(\mathrm{t}, J=8.0 \mathrm{~Hz}, 9 \mathrm{H}), 0.58(\mathrm{q}, J=8.2 \mathrm{~Hz}, 6 \mathrm{H}) \mathrm{ppm}$;

${ }^{13} \mathrm{C}$ NMR $\left(150 \mathrm{MHz}, \mathrm{CDCl}_{3}\right) \delta 210.5,148.3,141.8,138.3,134.3,129.8,123.2,115.0,111.1,70.4$, 53.6, 49.4, 47.1, 43.1, 35.3, 29.6, 23.7, 22.8, 20.3, 18.3, 7.2, 5.4 ppm;

HRMS (ESI): $\mathrm{m} / \mathrm{z}$ calcd for $\mathrm{C}_{26} \mathrm{H}_{45} \mathrm{O}_{2} \mathrm{Si}^{+}[\mathrm{M}+\mathrm{H}]^{+}:$: 417.3183, found: 417.3184;

IR (neat sample): 2958, 2875, 1716, 1456, 1374, 1238, 1058, 1007, 970, 892, $739 \mathrm{~cm}^{-1}$; 
$[\alpha]_{D}^{3:}+335.3\left(c 0.1, \mathrm{CHCl}_{3}\right)$.

\section{Synthesis of 19 via Stereoconvergent IMDA}

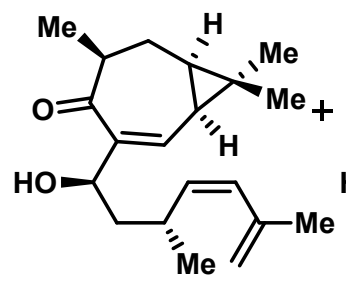

$7 c$

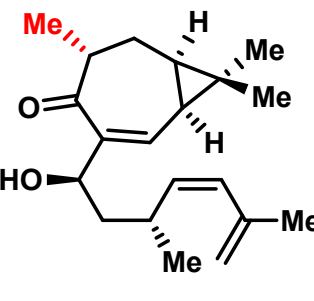

7b

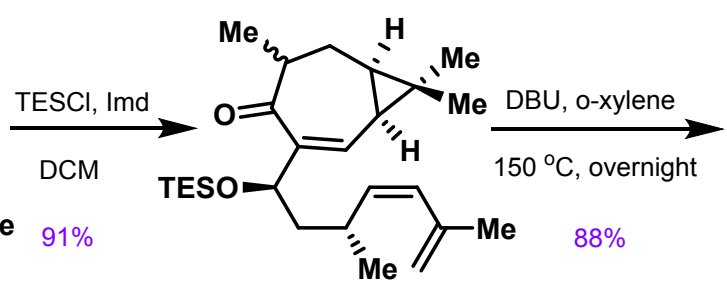

mixture of $17 \mathrm{c}$ and $17 \mathrm{~b}$

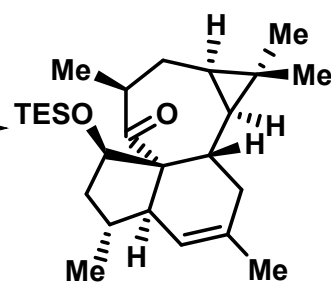

19

To a stirring solution of $7 \mathbf{c}$ and $7 \mathbf{b}$ (1.17 g, $3.87 \mathrm{mmol}, 1.00$ equiv) in $\mathrm{CH}_{2} \mathrm{Cl}_{2}(38.7 \mathrm{~mL}, 0.1 \mathrm{M})$ were added imidazole (791 mg, $11.6 \mathrm{mmol}, 3.00$ equiv), 4-dimethylaminopyridine (47.3 mg, 0.387 mmol, 0.100 equiv) and chlorotriethylsilane $\left(0.980 \mathrm{~mL}, 5.81 \mathrm{mmol}, 1.50\right.$ equiv) at $0{ }^{\circ} \mathrm{C}$ successively. Then the reaction was allowed to warm to room temperature and stirred for $30 \mathrm{~min}$ at this temperature. Then the saturated aq. $\mathrm{NH}_{4} \mathrm{Cl}$ was added to quench the reaction, and the mixture was extracted with $\mathrm{CH}_{2} \mathrm{Cl}_{2}$ three times. The combined organic layers were dried over $\mathrm{MgSO}_{4}$, filtered and concentrated in vacuo. The resultant residue was purified by column chromatography on silica gel $(P E / E A=60 / 1)$, yielding the mixture of $17 \mathrm{c}$ and $17 \mathrm{~b}(1.47 \mathrm{~g}, 91 \%)$ as a colourless oil.

1,8-Diazabicyclo[5.4.0]undec-7-ene (DBU, $0.640 \mathrm{~mL}, 4.24 \mathrm{mmol}, 1.20$ equiv) and the mixture of triene $17 \mathrm{c}$ and $17 \mathrm{~b}$ (1.47 g, $3.54 \mathrm{mmol}, 1.00$ equiv) were dissolved in o-xylene $(70.0 \mathrm{~mL}, 0.050$ $M$ ) in a sealed tube, and the solution was degassed with $\mathrm{N}_{2}$ via ultrasonic for $10 \mathrm{~min}$. Afterwards, the reaction was allowed to warm to $150{ }^{\circ} \mathrm{C}$ and stirred at this temperature overnight. Then the solvent was removed directly under reduced pressure, and the crude product was purified by flash column chromatography on silica gel $(P E / E A=60 / 1)$ to give product $19(1.30 \mathrm{~g}, 88 \%)$ as a colourless oil.

$\mathbf{R}_{\mathrm{f}}=0.52(\mathrm{PE} / \mathrm{EA}=20 / 1, \mathrm{CAM})$;

${ }^{1} \mathrm{H}$ NMR $\left(500 \mathrm{MHz}, \mathrm{CDCl}_{3}\right) \delta 5.47$ (ddd, $\left.J=5.4,2.9,1.5 \mathrm{~Hz}, 1 \mathrm{H}\right), 4.37$ (t, $\left.J=4.9 \mathrm{~Hz}, 1 \mathrm{H}\right), 2.97$ (d, $J=5.5 \mathrm{~Hz}, 1 \mathrm{H}), 2.95-2.87(\mathrm{~m}, 1 \mathrm{H}), 2.59-2.53(\mathrm{~m}, 1 \mathrm{H}), 2.42(\mathrm{ddd}, J=11.2,5.3,2.0 \mathrm{~Hz}, 1 \mathrm{H})$, $1.85(\mathrm{dq}, J=13.5,6.8 \mathrm{~Hz}, 1 \mathrm{H}), 1.80-1.73(\mathrm{~m}, 1 \mathrm{H}), 1.65(\mathrm{~d}, J=1.4 \mathrm{~Hz}, 3 \mathrm{H}), 1.56-1.45(\mathrm{~m}, 3 \mathrm{H})$, 1.33 (ddd, $J=12.5,7.8,5.0 \mathrm{~Hz}, 1 \mathrm{H}$ ), 1.27 (d, $J=7.5 \mathrm{~Hz}, 3 \mathrm{H}$ ), 1.07 (s, 3H), 1.01 (d, $J=6.9 \mathrm{~Hz}$, $3 \mathrm{H}), 0.96(\mathrm{t}, J=7.9 \mathrm{~Hz}, 9 \mathrm{H}), 0.92(\mathrm{~s}, 3 \mathrm{H}), 0.66-0.57(\mathrm{~m}, 8 \mathrm{H}) \mathrm{ppm}$; 
${ }^{13} \mathrm{C}$ NMR $\left(125 \mathrm{MHz}, \mathrm{CDCl}_{3}\right) \delta 216.7,130.3,124.7,77.8,67.3,52.7,45.2,42.4,40.0,34.8,31.5$, 29.1, 28.9, 28.5, 26.2, 24.6, 20.3, 19.3, 18.4, 14.6, 7.0, 5.3 ppm;

HRMS (ESI): $\mathrm{m} / \mathrm{z}$ calcd for $\mathrm{C}_{26} \mathrm{H}_{45} \mathrm{O}_{2} \mathrm{Si}^{+}[M+\mathrm{H}]^{+}:$: 417.3183, found: 417.3184;

IR (neat sample): 2954, 2875, 1678, 1457, 1413, 1376, 1237, 1103, 1050, 1003, 973, 902, 821, $720 \mathrm{~cm}^{-1}$;

$[\alpha]_{\mathrm{D}}^{3}:-77.4\left(c 1.15, \mathrm{CHCl}_{3}\right)$.

\section{Synthesis of 20}

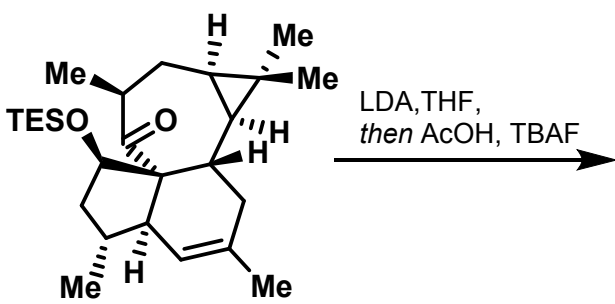

19

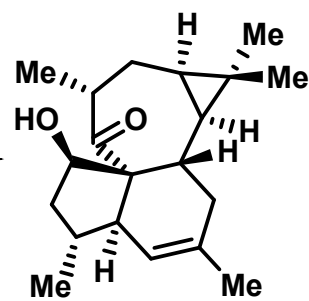

20

LDA preparation: diisopropylamine (2.06 mL, $14.7 \mathrm{mmol}, 5.30$ equiv) was dissolved in THF (29.0 mL) under $\mathrm{N}_{2}$ at $-78^{\circ} \mathrm{C}$. $n$-BuLi $(5.76 \mathrm{~mL}, 14.4 \mathrm{mmol}, 2.5 \mathrm{M}$ in hexane, 5.20 equiv) was added dropwise. The stirring was continued for $20 \mathrm{~min}$ at this temperature. Afterwards, the solution of ketone 19 (1.16 g, $2.78 \mathrm{mmol}, 1.00$ equiv) in THF $(29.0 \mathrm{~mL})$ was added dropwise via cannula at $-78^{\circ} \mathrm{C}$. After $50 \mathrm{~min}$, acetic acid $(0.830 \mathrm{~mL}, 14.4 \mathrm{mmol}, 5.20$ equiv) was added to quench the formed enolate at $-78{ }^{\circ} \mathrm{C}$, and the reaction was allowed to warm to $0{ }^{\circ} \mathrm{C}$ gradually. Then TBAF (28.8 $\mathrm{mL}, 28.8 \mathrm{mmol}, 1.0 \mathrm{M}$ in THF, 10.4 equiv) was added dropwise at $0{ }^{\circ} \mathrm{C}$. The cooling bath was removed to let the reaction slowly warm to room temperature and stir for $50 \mathrm{~min}$. Then the reaction was quenched by adding saturated aq. $\mathrm{NH}_{4} \mathrm{Cl}$ and diluted with $\mathrm{Et}_{2} \mathrm{O}$, extracted $\left(\mathrm{Et}_{2} \mathrm{O} \times\right.$ 3). The combined organic layers were dried over $\mathrm{MgSO}_{4}$, filtered and evaporated under reduced pressure. The remaining residue was purified by column chromatography on silica gel (PE/EA = 10/1), yielding alcohol 20 (0.840 g, quant.) as a colourless crystalline solid.

$\mathbf{R}_{\mathrm{f}}=0.38(\mathrm{PE} / \mathrm{EA}=6 / 1, \mathrm{CAM}) ;$

${ }^{1} \mathrm{H}$ NMR $\left(500 \mathrm{MHz}, \mathrm{CDCl}_{3}\right) \delta 5.52(\mathrm{tt}, J=2.7,1.4 \mathrm{~Hz}, 1 \mathrm{H}), 4.12(\mathrm{dd}, J=6.0,3.4 \mathrm{~Hz}, 1 \mathrm{H}), 3.16$ (ddq, $J=9.6,8.4,6.5 \mathrm{~Hz}, 1 \mathrm{H}$ ), 2.94 (h, $J=2.4 \mathrm{~Hz}, 1 \mathrm{H}$ ), 2.66 (ddtd, $J=16.9,6.1,2.6,1.3 \mathrm{~Hz}, 1 \mathrm{H}$ ), 2.41 (ddd, $J=11.4,6.0,1.6 \mathrm{~Hz}, 1 \mathrm{H}), 1.92$ (dtd, $J=8.9,7.0,5.0 \mathrm{~Hz}, 1 \mathrm{H}), 1.82$ (ddd, $J=14.6,8.4$, $4.9 \mathrm{~Hz}, 1 \mathrm{H}), 1.76-1.69(\mathrm{~m}, 2 \mathrm{H}), 1.69-1.65(\mathrm{~m}, 4 \mathrm{H}), 1.65-1.61(\mathrm{~m}, 1 \mathrm{H}), 1.36-1.29(\mathrm{~m}, 1 \mathrm{H})$, 
$1.11(\mathrm{~d}, J=6.9 \mathrm{~Hz}, 3 \mathrm{H}), 1.05(\mathrm{~s}, 3 \mathrm{H}), 1.03(\mathrm{~d}, J=6.5 \mathrm{~Hz}, 3 \mathrm{H}), 0.95(\mathrm{~s}, 3 \mathrm{H}), 0.63$ (dd, $J=11.4,9.2$ $\mathrm{Hz}, 1 \mathrm{H}), 0.54(\mathrm{td}, J=9.3,4.9 \mathrm{~Hz}, 1 \mathrm{H}) \mathrm{ppm}$;

${ }^{13} \mathrm{C}$ NMR $\left(125 \mathrm{MHz}, \mathrm{CDCl}_{3}\right) \delta 215.3,128.7,125.6,78.4,66.6,42.3,41.6,39.9,39.4,35.6,32.4$, 31.6, 29.8, 29.2, 24.3, 22.9, 19.6, 18.3, 18.2, 15.0 ppm;

HRMS (ESI): $\mathrm{m} / \mathrm{z}$ calcd for $\mathrm{C}_{20} \mathrm{H}_{29} \mathrm{O}^{+}\left[\mathrm{M}-\mathrm{H}_{2} \mathrm{O}+\mathrm{H}\right]^{+}:$:285.2213, found: 285.2213;

IR (neat sample): 3472, 2954, 2927, 2868, 1695, 1454, 1375, 1320, 1189, 1137, 1079, 1052, 1002, 947, 856, $832 \mathrm{~cm}^{-1}$;

$[\alpha]_{D}^{3:}-82.8\left(c \quad 0.7, \mathrm{CHCl}_{3}\right)$.

\section{One-pot Synthesis of 20}

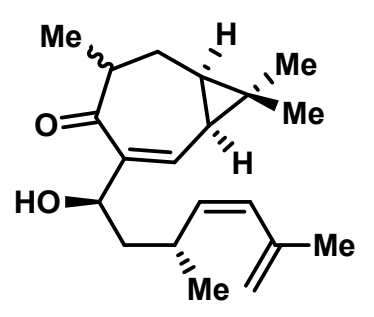

mixture of $7 c$ and $7 b$

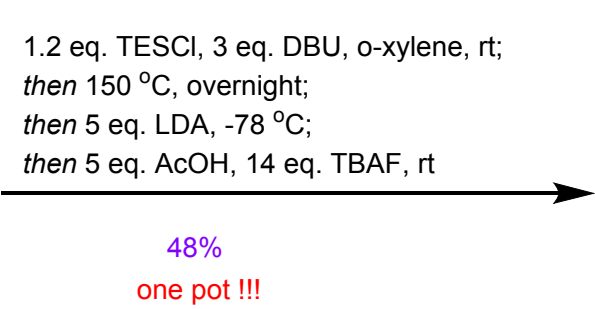

one pot !!!

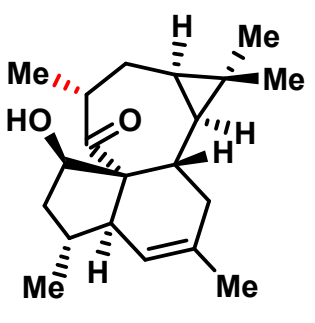

20

A $25 \mathrm{~mL}$ sealed tube with rubber septum was charged with the mixture of $10 \mathrm{~b}$ and $10 \mathrm{c}$ (33.0 $\mathrm{mg}, 0.110 \mathrm{mmol}, 1.00$ equiv) in degassed o-xylene $(2.20 \mathrm{~mL}, 0.050 \mathrm{M})$. To the stirring mixture were added 1,8-Diazabicyclo[5.4.0]undec-7-ene (DBU, $50.0 \mu \mathrm{L}, 0.330 \mathrm{mmol}, 3.00$ equiv) and chlorotriethylsilane $(22.0 \mu \mathrm{L}, 0.131 \mathrm{mmol}, 1.20$ equiv) successively at room temperature. The reaction was stirred for $20 \mathrm{~min}$ before ethanol (1.34 $\mu \mathrm{L}, 33.0 \mu \mathrm{mol}, 0.300$ equiv) was added to consume the excessive chlorotriethylsilane. Then the rubber septum was changed to the original Teflon cap, and the reaction was warmed to $150{ }^{\circ} \mathrm{C}$ and stirred overnight. After TLC showed the full conversion of triene, the reaction was cooled to room temperature, and the Teflon cap was changed to rubber septum again. After 3-cycle pumping and refilling with $\mathrm{N}_{2}$, the reaction mixture was cooled to $-78^{\circ} \mathrm{C}$ and $2.20 \mathrm{~mL}$ THF was added. Afterwards, the freshly prepared LDA solution (1.10 mL, $0.550 \mathrm{mmol}, 0.50 \mathrm{M}$ in THF, 5.00 equiv) was added dropwise at $-78{ }^{\circ} \mathrm{C}$. After $50 \mathrm{~min}$, acetic acid $(31.0 \mu \mathrm{L}, 0.550 \mathrm{mmol}, 5.00$ equiv) was added to quench the formed enolate at -78 ${ }^{\circ} \mathrm{C}$, and the reaction was allowed to warm to $0{ }^{\circ} \mathrm{C}$ gradually. Then TBAF (1.54 mL, $1.54 \mathrm{mmol}, 1.0$ $\mathrm{M}$ in THF, 14.0 equiv) was added dropwise at $0^{\circ} \mathrm{C}$. The cooling bath was then removed to let the reaction slowly warm to room temperature and stir for $50 \mathrm{~min}$. Then the reaction was quenched 
by adding saturated aq. $\mathrm{NH}_{4} \mathrm{Cl}$ and diluted with $\mathrm{Et}_{2} \mathrm{O}$, extracted $\left(\mathrm{Et}_{2} \mathrm{O} \times 3\right)$. The combined organic layers were dried over $\mathrm{MgSO}_{4}$, filtered and evaporated under reduced pressure. The remaining residue was purified by column chromatography on silica gel (PE/EA $=15 / 1$ to $12 / 1)$, yielding alcohol 20 (16.0 mg, 48\%) as a colourless crystalline solid.

$\mathbf{R}_{\mathbf{f}}=0.38(\mathrm{PE} / \mathrm{EA}=6 / 1, \mathrm{CAM})$;

${ }^{1} \mathrm{H}$ NMR $\left(500 \mathrm{MHz}, \mathrm{CDCl}_{3}\right) \delta 5.52(\mathrm{tt}, J=2.7,1.4 \mathrm{~Hz}, 1 \mathrm{H}), 4.12(\mathrm{dd}, J=6.0,3.4 \mathrm{~Hz}, 1 \mathrm{H}), 3.16$ (ddq, $J=9.6,8.4,6.5 \mathrm{~Hz}, 1 \mathrm{H}$ ), 2.94 (h, $J=2.4 \mathrm{~Hz}, 1 \mathrm{H}$ ), 2.66 (ddtd, $J=16.9,6.1,2.6,1.3 \mathrm{~Hz}, 1 \mathrm{H}$ ), 2.41 (ddd, $J=11.4,6.0,1.6 \mathrm{~Hz}, 1 \mathrm{H}$ ), 1.92 (dtd, $J=8.9,7.0,5.0 \mathrm{~Hz}, 1 \mathrm{H}$ ), 1.82 (ddd, $J=14.6,8.4$, $4.9 \mathrm{~Hz}, 1 \mathrm{H}), 1.76-1.69(\mathrm{~m}, 2 \mathrm{H}), 1.69-1.65(\mathrm{~m}, 4 \mathrm{H}), 1.65-1.61(\mathrm{~m}, 1 \mathrm{H}), 1.36-1.29(\mathrm{~m}, 1 \mathrm{H})$, $1.11(\mathrm{~d}, J=6.9 \mathrm{~Hz}, 3 \mathrm{H}), 1.05(\mathrm{~s}, 3 \mathrm{H}), 1.03(\mathrm{~d}, J=6.5 \mathrm{~Hz}, 3 \mathrm{H}), 0.95$ (s, 3H), 0.63 (dd, J = 11.4, 9.2 $\mathrm{Hz}, 1 \mathrm{H}), 0.54$ (td, $J=9.3,4.9 \mathrm{~Hz}, 1 \mathrm{H}) \mathrm{ppm}$;

${ }^{13} \mathrm{C}$ NMR $\left(125 \mathrm{MHz}, \mathrm{CDCl}_{3}\right) \delta 215.3,128.7,125.6,78.4,66.6,42.3,41.6,39.9,39.4,35.6,32.4$, 31.6, 29.8, 29.2, 24.3, 22.9, 19.6, 18.3, 18.2, 15.0 ppm;

HRMS (ESI): $\mathrm{m} / \mathrm{z}$ calcd for $\mathrm{C}_{20} \mathrm{H}_{29} \mathrm{O}^{+}\left[M-\mathrm{H}_{2} \mathrm{O}+\mathrm{H}\right]^{+}:$: 285.2213, found: 285.2213;

IR (neat sample): 3472, 2954, 2927, 2868, 1695, 1454, 1375, 1320, 1189, 1137, 1079, 1052 , 1002, 947, 856, $832 \mathrm{~cm}^{-1}$;

$[\alpha]_{D}^{3:}-82.8\left(c 0.7, \mathrm{CHCl}_{3}\right)$.

\section{Synthesis of 21}

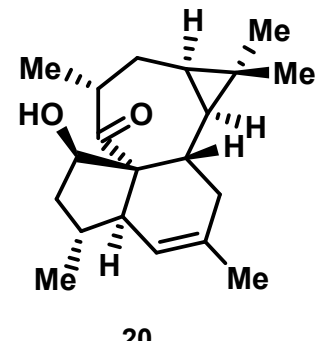

20

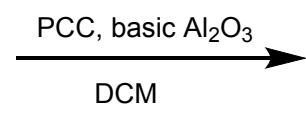

$77 \%$

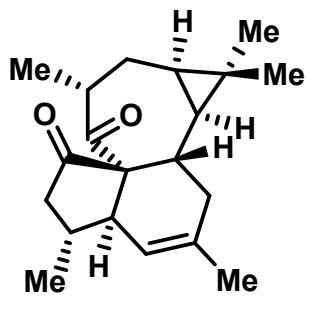

21

To the stirring solution of alcohol $20\left(0.870 \mathrm{~g}, 2.88 \mathrm{mmol}, 1.00\right.$ equiv) were added basic $\mathrm{Al}_{2} \mathrm{O}_{3}$ $(9.93 \mathrm{~g})$ and pyridinium chlorochromate $(2.48 \mathrm{~g}, 11.5 \mathrm{mmol}, 4.00$ equiv) successively at room temperature. The reaction was stirred for $30 \mathrm{~min}$ before it was filtered through a short pad of silica gel and washed with $\mathrm{CH}_{2} \mathrm{Cl}_{2}$ three times. The resultant filtrate was concentrated under reduced pressure. The obtained solid was purified by column chromatography on silica gel (PE/EA = 15/1), yielding diketone 21 (660 mg, 77\%) as a colourless crystalline solid. 
$\mathbf{R}_{\mathbf{f}}=0.55(\mathrm{PE} / \mathrm{EA}=10 / 1, \mathrm{CAM})$;

${ }^{1} \mathrm{H}$ NMR $\left(400 \mathrm{MHz}, \mathrm{CDCl}_{3}\right) \delta 5.52(\mathrm{td}, J=2.9,1.4 \mathrm{~Hz}, 1 \mathrm{H}), 3.43(\mathrm{tq}, J=8.9,6.5 \mathrm{~Hz}, 1 \mathrm{H}), 3.35-$ $3.29(\mathrm{~m}, 1 \mathrm{H}), 2.62$ (ddd, $J=11.3,5.6,1.8 \mathrm{~Hz}, 1 \mathrm{H}), 2.34$ (dd, $J=17.9,8.4 \mathrm{~Hz}, 1 \mathrm{H}), 2.16-1.98(\mathrm{~m}$, $2 \mathrm{H}), 1.73-1.60(\mathrm{~m}, 5 \mathrm{H}), 1.11(\mathrm{dd}, J=7.2,0.6 \mathrm{~Hz}, 3 \mathrm{H}), 1.07(\mathrm{~s}, 3 \mathrm{H}), 0.96(\mathrm{~d}, 3 \mathrm{H}), 0.94(\mathrm{~s}, 3 \mathrm{H})$, $0.64-0.52(\mathrm{~m}, 2 \mathrm{H}) \mathrm{ppm}$;

${ }^{13} \mathrm{C}$ NMR $\left(100 \mathrm{MHz}, \mathrm{CDCl}_{3}\right) \delta 215.2,208.4,129.8,124.4,73.1,45.5,41.9,38.7,35.6,35.6,32.7$, 30.0, 29.7, 29.0, 24.3, 22.6, 21.5, 18.7, 17.9, 14.8 ppm;

HRMS (ESI): $\mathrm{m} / \mathrm{z}$ calcd for $\mathrm{C}_{20} \mathrm{H}_{29} \mathrm{O}_{2}{ }^{+}[M+\mathrm{H}]^{+}:$301.2162, found: 301.2159;

IR (neat sample): 2925, 2873, 1730, 1703, 1453, 1372, 1246, 1144, 1122, 1071, 1011, 963, 937, $801,735,711 \mathrm{~cm}^{-1}$;

$[\alpha]_{D}^{3}:-255.6\left(c 0.95, \mathrm{CHCl}_{3}\right)$.

\section{Synthesis of 22}

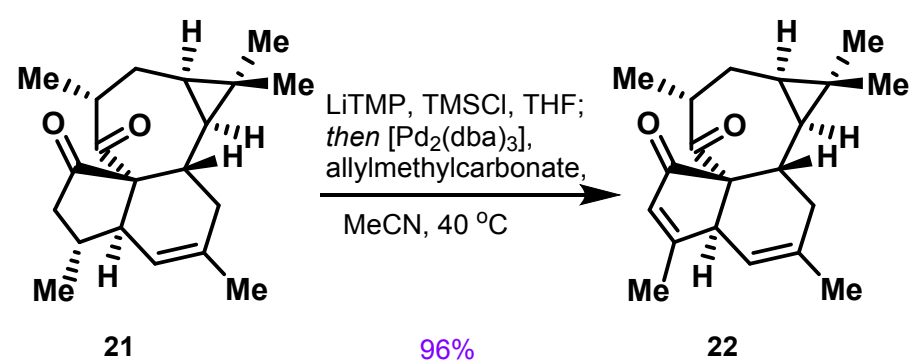

2,2,6,6-Tetramethylpiperidine ( $1.76 \mathrm{~mL}, 10.3 \mathrm{mmol}, 5.20$ equiv) was dissolved in dry THF (20.6 $\mathrm{mL}$ ). The solution was cooled to $-78^{\circ} \mathrm{C}$, and $\mathrm{n}$-BuLi (3.96 mL, $2.5 \mathrm{M}$ in hexane, $9.90 \mathrm{mmol}, 5.00$ equiv) was added dropwise at this temperature. The reaction was kept stirring for $10 \mathrm{~min}$. Then the freshly prepared Lithium tetramethylpiperidide was slowly transferred to the stirring solution mixed with diketone 21 (596 mg, 1.98 mmol, 1.00 equiv) and TMSCl (1.00 mL, 7.92 mmol, 4.00 equiv) in THF $(20.6 \mathrm{~mL})$ via cannula at $-78^{\circ} \mathrm{C}$. After stirring for $30 \mathrm{~min}$ at $-78^{\circ} \mathrm{C}$, the reaction was warmed to $0^{\circ} \mathrm{C}$ gradually. The stirring was continued at $0{ }^{\circ} \mathrm{C}$ for $10 \mathrm{~min}$ before it was diluted with pentane and quenched by saturated aq. $\mathrm{NaHCO}_{3}$. Then the reaction was extracted with pentane three times. The combined organic layers were dried over $\mathrm{MgSO}_{4}$, filtered and evaporated under reduced pressure. The remaining crude TMS-enol ether was re-dissolved in dry MeCN (40.0 mL, $0.050 \mathrm{M}$ ). To the above stirring solution were added tris(dibenzylideneacetone)dipalladium(0) (362 $\mathrm{mg}, 0.396 \mathrm{mmol}, 0.200$ equiv) in one portion and allyl methyl carbonate $(0.450 \mathrm{~mL}, 3.96 \mathrm{mmol}$, 2.00 equiv) dropwise at room temperature. The reaction was allowed to warm to $40{ }^{\circ} \mathrm{C}$ and stirred 
for $3 \mathrm{~h}$ before it was filtered through a short pad of Celite ${ }^{\circledR}$. The filtrate was collected and concentrated in vacuo. The residue was purified by column chromatography on silica gel (PE/EA $=30 / 1)$ to give enone 22 (566 mg, 96\%) as a slightly yellow solid.

$\mathbf{R}_{\mathrm{f}}=0.21(\mathrm{PE} / \mathrm{EA}=20 / 1, \mathrm{CAM})$;

${ }^{1} \mathrm{H}$ NMR $\left(400 \mathrm{MHz}, \mathrm{CDCl}_{3}\right) \delta 5.61-5.54(\mathrm{~m}, 1 \mathrm{H}), 5.51$ (ddd, $\left.J=4.4,2.9,1.5 \mathrm{~Hz}, 1 \mathrm{H}\right), 3.86(\mathrm{~s}$, 1H), 3.39 (ddq, $J=10.1,8.3,6.4 \mathrm{~Hz}, 1 \mathrm{H}$ ), 2.82 (ddd, $J=11.5,5.3,2.0 \mathrm{~Hz}, 1 \mathrm{H}$ ), $2.28-2.17$ (m, 4H), 1.91 (ddd, $J=14.6,8.3,4.5 \mathrm{~Hz}, 1 \mathrm{H}), 1.73-1.60(\mathrm{~m}, 5 \mathrm{H}), 1.11(\mathrm{~s}, 3 \mathrm{H}), 0.97(\mathrm{~s}, 3 \mathrm{H}), 0.91$ (d, $J=6.4 \mathrm{~Hz}, 3 \mathrm{H}$ ), 0.70 (dd, $J=11.5,9.2 \mathrm{~Hz}, 1 \mathrm{H}), 0.55$ (td, $J=9.3,4.5 \mathrm{~Hz}, 1 \mathrm{H}$ ) ppm;

${ }^{13} \mathrm{C}$ NMR $\left(100 \mathrm{MHz}, \mathrm{CDCl}_{3}\right) \delta$ 208.2, 203.6, 183.9, 133.7, 124.7, 118.6, 74.9, 46.8, 38.9, 33.5, 33.1, 29.6, 29.5, 29.1, 24.6, 22.1, 18.6, 18.3, 17.6, 14.8 ppm;

HRMS (ESI): $\mathrm{m} / \mathrm{z}$ calcd for $\mathrm{C}_{20} \mathrm{H}_{27} \mathrm{O}_{2}{ }^{+}[M+\mathrm{H}]^{+}:$299.2006, found: 299.2003;

IR (neat sample): 2969, 2919, 2856, 1685, 1619, 1434, 1377, 1308, 1282, 1249, 1169, 1123, 1006, 941, 835, 853, 803, 765, 748, $692 \mathrm{~cm}^{-1}$;

$[\alpha]_{\mathrm{D}}^{3}:-29.4\left(c 0.95, \mathrm{CHCl}_{3}\right)$.

\section{Synthesis of (+)-Pepluanol A (1), 23 and 24}

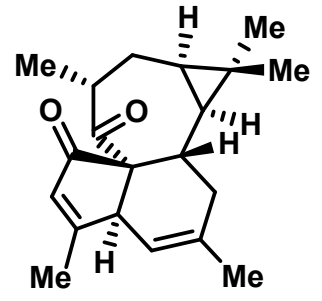

22

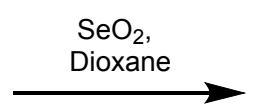

$60{ }^{\circ} \mathrm{C}, 8 \mathrm{~h}$ recovered SM: $28 \%$

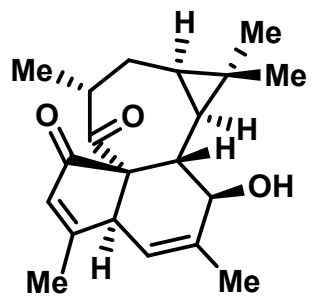

(+)-Pepluanol A (1)

$37 \%$ brsm: $52 \%$

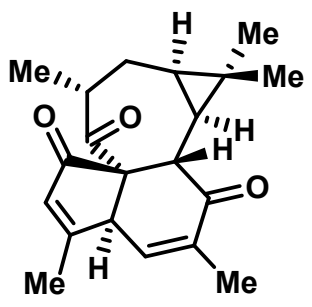

24 $16 \%$

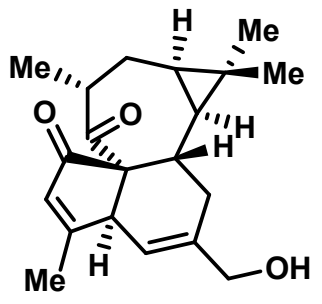

23

$18 \%$

For allylic oxidation with $\mathrm{SeO}_{2}$, the extremely anhydrous procedure was adopted. $\mathrm{SeO}_{2}(370 \mathrm{mg}$, $3.35 \mathrm{mmol}, 10.0$ equiv) was put in a Schlenk flask. Then the flask was warmed to $60{ }^{\circ} \mathrm{C}$ in vacuo for $1 \mathrm{~h}$. After refilling with $\mathrm{N}_{2}$, the solution of alkene 22 (100 mg, $0.335 \mathrm{mmol}, 1.00$ equiv) in dry 1,4-dioxane $(3.40 \mathrm{~mL}, 0.10 \mathrm{M})$ was added to the above dry Schlenk flask under $\mathrm{N}_{2}$ at room temperature. Then the reaction was warmed to $60{ }^{\circ} \mathrm{C}$ and stirred for $8 \mathrm{~h}$. The resulting yellow suspension was filtered through a short pad of silica and washed with ethyl acetate. The filtrate was collected and concentrated in vacuo. The resultant residue was submitted to a long column packed with silica gel eluting with pentane/ $\mathrm{Et}_{2} \mathrm{O}(10 / 1$ to $5 / 1$ to $2 / 1$ to $1 / 1)$ to give recovered 22 
(28.0 mg, 28\%, slightly yellow solid), 24 (16.0 mg, 16\%, slightly yellow solid), 23 (18.0 mg, 18\%, white solid) and (+)-pepluanol A (1) (37.3 mg, 37\%, white solid), respectively.

\section{4:}

$\mathbf{R}_{\mathbf{f}}=0.23(\mathrm{PE} / \mathrm{EA}=5 / 1, \mathrm{CAM})$;

${ }^{1} \mathrm{H}$ NMR (400 MHz, CDCl $)$ $\delta 6.59(\mathrm{dp}, J=4.1,1.5 \mathrm{~Hz}, 1 \mathrm{H}), 5.70(\mathrm{p}, J=1.3 \mathrm{~Hz}, 1 \mathrm{H}), 4.10$ (dhept, $J=4.0,1.4 \mathrm{~Hz}, 1 \mathrm{H}), 3.43(\mathrm{~d}, J=11.3 \mathrm{~Hz}, 1 \mathrm{H}), 3.26$ (tq, $J=8.8,6.4 \mathrm{~Hz}, 1 \mathrm{H}), 2.33$ (dd, $J=1.4,0.8$ $\mathrm{Hz}, 3 \mathrm{H}), 1.99$ (ddd, $J=14.3,8.4,5.5 \mathrm{~Hz}, 1 \mathrm{H}), 1.79$ (t, $J=1.7 \mathrm{~Hz}, 3 \mathrm{H}), 1.73$ (dt, $J=14.8,8.8 \mathrm{~Hz}$, $1 \mathrm{H}), 1.25(\mathrm{~s}, 3 \mathrm{H}), 0.99-0.89(\mathrm{~m}, 6 \mathrm{H}), 0.75-0.60(\mathrm{~m}, 2 \mathrm{H}) \mathrm{ppm}$;

${ }^{13} \mathrm{C}$ NMR $\left(100 \mathrm{MHz}, \mathrm{CDCl}_{3}\right) \delta$ 205.7, 200.6, 198.2, 181.6, 139.5, 134.0, 126.0, 75.8, 47.1, 45.0, 39.5, 30.7, 29.1, 28.5, 23.2, 19.9, 18.3, 17.5, 16.7, 15.5 ppm;

HRMS (ESI): $\mathrm{m} / \mathrm{z}$ calcd for $\mathrm{C}_{20} \mathrm{H}_{25} \mathrm{O}_{3}{ }^{+}[\mathrm{M}+\mathrm{H}]^{+}:$313.1798, found: 315.1794 ;

IR (neat sample): 2927, 1694, 1622, 1451, 1376, 1298, 1240, 1177, 1124, 1098, 1033, 948, 911 , $845,730 \mathrm{~cm}^{-1}$;

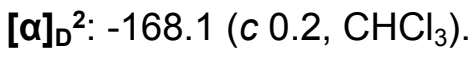

23:

$\mathbf{R}_{\mathbf{f}}=0.36\left(\right.$ Pentane/Et $\left.{ }_{2} \mathrm{O}=1 / 2, \mathrm{CAM}\right)$;

${ }^{1} \mathrm{H}$ NMR $\left(600 \mathrm{MHz}, \mathrm{CDCl}_{3}\right) \delta 5.83$ (ddd, $\left.J=4.6,3.0,1.5 \mathrm{~Hz}, 1 \mathrm{H}\right), 5.61(\mathrm{t}, J=1.4 \mathrm{~Hz}, 1 \mathrm{H}), 4.05-$ $3.93(\mathrm{~m}, 3 \mathrm{H}), 3.44-3.36(\mathrm{~m}, 1 \mathrm{H}), 2.89(\mathrm{ddd}, J=11.6,5.3,2.1 \mathrm{~Hz}, 1 \mathrm{H}), 2.24(\mathrm{t}, J=1.0 \mathrm{~Hz}, 3 \mathrm{H})$, $2.22-2.16(\mathrm{~m}, 1 \mathrm{H}), 1.92$ (ddd, $J=14.7,8.4,4.5 \mathrm{~Hz}, 1 \mathrm{H}), 1.84(\mathrm{dd}, J=16.6,2.0 \mathrm{~Hz}, 1 \mathrm{H}), 1.68$ (dt, $J=14.7,9.8 \mathrm{~Hz}, 1 \mathrm{H}), 1.62(\mathrm{t}, J=4.4 \mathrm{~Hz}, 1 \mathrm{H}), 1.12(\mathrm{~s}, 3 \mathrm{H}), 0.97(\mathrm{~s}, 3 \mathrm{H}), 0.93(\mathrm{~d}, J=6.4 \mathrm{~Hz}, 3 \mathrm{H})$, 0.67 (dd, $J=11.5,9.2 \mathrm{~Hz}, 1 \mathrm{H}), 0.56$ (td, $J=9.3,4.5 \mathrm{~Hz}, 1 \mathrm{H}) \mathrm{ppm}$;

${ }^{13} \mathrm{C}$ NMR $\left(150 \mathrm{MHz}, \mathrm{CDCl}_{3}\right) \delta 207.9,203.3,183.2,136.8,125.2,119.2,75.5,66.8,46.4,39.0$, 33.3, 29.5, 29.3, 29.0, 28.8, 22.1, 18.8, 18.4, 17.6, 14.8 ppm;

HRMS (ESI): $\mathrm{m} / \mathrm{z}$ calcd for $\mathrm{C}_{20} \mathrm{H}_{27} \mathrm{O}_{3}{ }^{+}[M+\mathrm{H}]^{+}:$315.1955, found: 315.1954;

IR (neat sample): 3419, 2926, 2855, 1688, 1620, 1434, 1375, 1305, 1183, 1123, 1061, 1006, 942, $841,733,688 \mathrm{~cm}^{-1}$;

$[\alpha]_{D}^{3:}-39.9\left(c 0.55, \mathrm{CHCl}_{3}\right)$.

\section{(+)-pepluanol A (1):}

$\mathbf{R}_{\mathbf{f}}=0.28$ (Pentane/Et $\left.{ }_{2} \mathrm{O}=1 / 2, \mathrm{CAM}\right)$;

${ }^{1} \mathrm{H}$ NMR $\left(400 \mathrm{MHz}, \mathrm{CDCl}_{3}\right) \delta 5.70(\mathrm{dd}, J=4.7,1.6 \mathrm{~Hz}, 1 \mathrm{H}), 5.65(\mathrm{t}, J=1.4 \mathrm{~Hz}, 1 \mathrm{H}), 3.95(\mathrm{brs}, 1 \mathrm{H})$, $3.89(\mathrm{~d}, J=2.5 \mathrm{~Hz}, 1 \mathrm{H}), 3.54-3.43(\mathrm{~m}, 1 \mathrm{H}), 2.95(\mathrm{dd}, J=11.8,2.6 \mathrm{~Hz}, 1 \mathrm{H}), 2.23$ (brs, 3H), 1.95 
(ddd, $J=14.7,8.5,5.1 \mathrm{~Hz}, 1 \mathrm{H}), 1.81(\mathrm{t}, J=1.7 \mathrm{~Hz}, 4 \mathrm{H}$, overlap with $-\mathrm{OH}$ ), 1.70 (dt, $J=14.6,9.4$ $\mathrm{Hz}, 1 \mathrm{H}), 1.16(\mathrm{~s}, 3 \mathrm{H}), 0.97(\mathrm{~s}, 3 \mathrm{H}), 0.94(\mathrm{~d}, J=6.5 \mathrm{~Hz}, 3 \mathrm{H}), 0.60(\mathrm{td}, J=9.2,5.1 \mathrm{~Hz}, 1 \mathrm{H}), 0.35$ (dd, $J=11.8,9.3 \mathrm{~Hz}, 1 \mathrm{H}$ ) ppm;

${ }^{13} \mathrm{C}$ NMR $\left(100 \mathrm{MHz}, \mathrm{CDCl}_{3}\right) \delta$ 207.6, 203.7, 182.0, 134.7, 125.2, 122.7, 73.6, 71.7, 46.7, 41.9, $39.4,29.3,28.9,28.4,22.7,22.3,18.8,18.5,17.7,15.0$ ppm;

HRMS (ESI): $\mathrm{m} / \mathrm{z}$ calcd for $\mathrm{C}_{20} \mathrm{H}_{25} \mathrm{O}_{2}{ }^{+}\left[M-\mathrm{H}_{2} \mathrm{O}+\mathrm{H}\right]^{+}:$297.1845, found: 297.1845;

IR (neat sample): 3425, 2967, 2932, 1674, 1623, 1450, 1436, 1375, 1303, 1274, 1184, 1124, 1110, 1072, 1024, 993, 946, 916, 846, 731, $688 \mathrm{~cm}^{-1}$.

$[\alpha]_{D}{ }^{4:}+70.7(c 1.3, \mathrm{MeOH})$; for isolated sample $[\alpha]_{D}{ }^{5}:+68.3(c 0.27, \mathrm{MeOH}) .{ }^{[3]}$ 
Table 1. ${ }^{1} \mathbf{H}$ NMR Chemical Shifts Comparison of Natural and Synthetic (+)-Pepluanol A

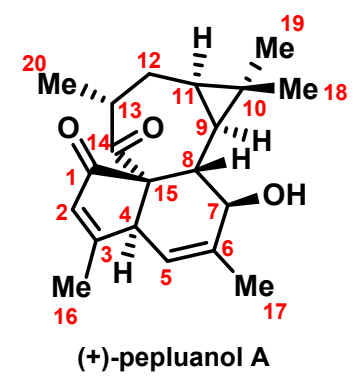

\begin{tabular}{|c|c|c|c|}
\hline Number & $\begin{array}{l}\text { Ding }^{[4]}\left(400 \mathrm{MHz}, \mathrm{CDCl}_{3}\right) \\
\delta^{1} \mathrm{H}[\mathrm{ppm}, \text { mult, } J(\mathrm{~Hz})]\end{array}$ & $\begin{array}{c}\text { Natural[3] }(600 \mathrm{MHz}, \\
\left.\mathrm{CDCl}_{3}\right) \\
\delta^{1} \mathrm{H}[\mathrm{ppm}, \text { mult, } J(\mathrm{~Hz})]\end{array}$ & $\begin{array}{l}\left.\text { Gaich (400 MHz, } \mathrm{CDCl}_{3}\right) \\
\delta^{1} \mathrm{H}[\mathrm{ppm}, \text { mult, } J(\mathrm{~Hz})]\end{array}$ \\
\hline 1 & --- & --- & --- \\
\hline 2 & $5.70-5.62 \mathrm{~m}$ & 5.66 brs & $5.65 \mathrm{t}(\mathrm{J}=1.4 \mathrm{~Hz})$ \\
\hline 3 & --- & --- & --- \\
\hline 4 & 3.96 brs & 3.95 brs & 3.95 brs \\
\hline 5 & $5.74-5.70 \mathrm{~m}$ & $5.71 \mathrm{brd}$ & $5.70 \mathrm{dd}(J=4.7,1.6 \mathrm{~Hz})$ \\
\hline 6 & --- & --- & --- \\
\hline 7 & $3.89 \mathrm{dd}(\mathrm{J}=3.6,2.7 \mathrm{~Hz})$ & 3.89 brs & $3.89 \mathrm{~d}(J=2.5 \mathrm{~Hz})$ \\
\hline 8 & $2.95 \mathrm{dd}(J=11.8,2.5 \mathrm{~Hz})$ & $2.95 \mathrm{dd}(\mathrm{J}=11.8,2.2 \mathrm{~Hz})$ & $2.95 \mathrm{dd}(\mathrm{J}=11.8,2.6 \mathrm{~Hz})$ \\
\hline 9 & $0.36 \mathrm{dd}(J=11.8,9.3 \mathrm{~Hz})$ & $0.35 \mathrm{dd}(\mathrm{J}=11.8,9.4 \mathrm{~Hz})$ & $0.35 \mathrm{dd}(\mathrm{J}=11.8,9.3 \mathrm{~Hz})$ \\
\hline 10 & --- & --- & --- \\
\hline 11 & $0.61 \mathrm{td}(\mathrm{J}=9.2,5.2 \mathrm{~Hz})$ & $0.61 \mathrm{td}(J=9.4,5.2 \mathrm{~Hz})$ & $0.60 \mathrm{td}(\mathrm{J}=9.2,5.1 \mathrm{~Hz})$ \\
\hline \multirow[t]{2}{*}{12} & 2.01-1.92 m; & $1.96 \mathrm{~m}$ & $\begin{array}{c}1.95 \text { ddd }(J=14.7,8.5 \\
5.1 \mathrm{~Hz})\end{array}$ \\
\hline & $1.75-1.65 \mathrm{~m}$ & $1.70 \mathrm{~m}$ & $1.70 \mathrm{dt}(\mathrm{J}=14.6,9.4 \mathrm{~Hz})$ \\
\hline 13 & 3.54-3.44 m & $3.49 \mathrm{~m}$ & $3.54-3.43 \mathrm{~m}$ \\
\hline 14 & --- & --- & --- \\
\hline 15 & --- & --- & --- \\
\hline 16 & $2.25 \mathrm{brs}$ & $2.24 \mathrm{brs}$ & $2.23 \mathrm{brs}$ \\
\hline 17 & $1.83 \mathrm{t}(J=1.6 \mathrm{~Hz})$ & $1.82 \mathrm{brs}$ & $1.81 \mathrm{t}(\mathrm{J}=1.7 \mathrm{~Hz})$ \\
\hline 18 & $0.98 \mathrm{~s}$ & $0.97 \mathrm{~s}$ & $0.97 \mathrm{~s}$ \\
\hline 19 & $1.17 \mathrm{~s}$ & $1.17 \mathrm{~s}$ & $1.16 \mathrm{~s}$ \\
\hline 20 & $0.95 \mathrm{~d}(J=6.4 \mathrm{~Hz})$ & $0.94 \mathrm{~d}(J=6.4 \mathrm{~Hz})$ & $0.94 \mathrm{~d}(J=6.5 \mathrm{~Hz})$ \\
\hline
\end{tabular}


Table 2. ${ }^{13} \mathrm{C}$ NMR Chemical Shifts Comparison of Natural and Synthetic (+)-Pepluanol A

\begin{tabular}{|c|c|c|c|}
\hline \multicolumn{4}{|c|}{ (+)-pepluanol A } \\
\hline Number & $\begin{array}{l}\text { Ding }^{[4]}\left(400 \mathrm{MHz} \mathrm{CDCl}_{3}\right) \\
\delta^{13} \mathrm{C}[\mathrm{ppm}, \text { mult, } J(\mathrm{~Hz})]\end{array}$ & $\begin{array}{c}\text { Natural[3] } \\
\left(150 \mathrm{MHz}^{[3 \mathrm{CDCl}}\right)_{3} \\
\delta 13 \mathrm{C}[\mathrm{ppm}, \text { mult, } \mathrm{J}(\mathrm{Hz})]\end{array}$ & $\begin{array}{l}\left.\text { Gaich (100 MHz, } \mathrm{CDCl}_{3}\right) \\
\delta^{13} \mathrm{C}[\mathrm{ppm}, \text { mult, } J(\mathrm{~Hz})]\end{array}$ \\
\hline 1 & 203.4 & 203.5 & 203.7 \\
\hline 2 & 125.1 & 125.1 & 125.2 \\
\hline 3 & 181.8 & 181.8 & 182.0 \\
\hline 4 & 46.6 & 46.5 & 46.7 \\
\hline 5 & 122.7 & 122.7 & 122.7 \\
\hline 6 & 134.6 & 134.5 & 134.7 \\
\hline 7 & 73.6 & 73.5 & 73.6 \\
\hline 8 & 41.7 & 41.7 & 41.9 \\
\hline 9 & 28.4 & 28.3 & 28.4 \\
\hline 10 & 18.8 & 18.7 & 18.8 \\
\hline 11 & 22.3 & 22.2 & 22.3 \\
\hline 12 & 29.2 & 29.2 & 29.3 \\
\hline 13 & 39.3 & 39.3 & 39.4 \\
\hline 14 & 207.3 & 207.4 & 207.6 \\
\hline 15 & 71.7 & 71.6 & 71.7 \\
\hline 16 & 18.3 & 18.3 & 18.5 \\
\hline 17 & 22.5 & 22.5 & 22.7 \\
\hline 18 & 28.8 & 28.8 & 28.9 \\
\hline 19 & 14.9 & 14.9 & 15.0 \\
\hline 20 & 17.6 & 17.6 & 17.7 \\
\hline
\end{tabular}




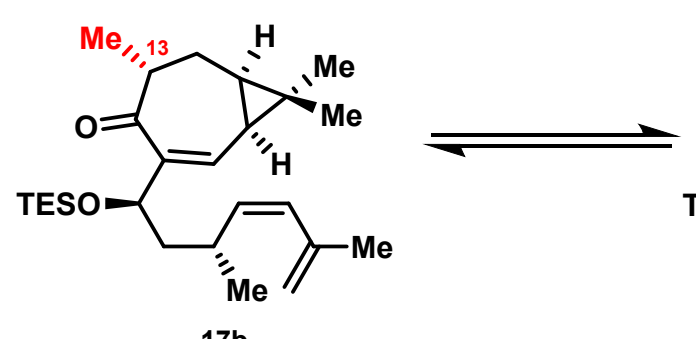

$17 \mathrm{~b}$

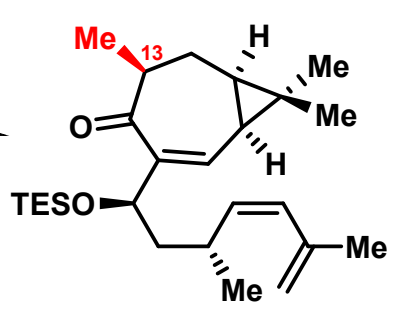

$17 \mathrm{c}$

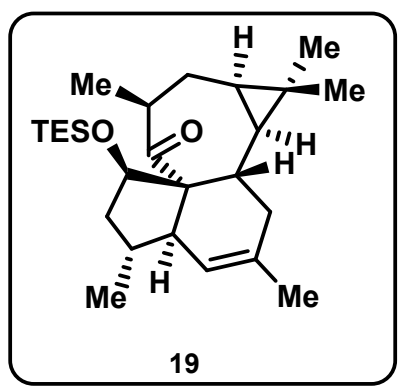

19

After screening the appropriate temperature for the epimerization before IMDA, we found that epimerization can be triggered above $100^{\circ} \mathrm{C}$. (extremely slow epimerization below $100{ }^{\circ} \mathrm{C}$ ) Therefore, 17b and 17c were subjected to the exactly same conditions (1.20 eq. DBU, o-xylene, $100{ }^{\circ} \mathrm{C}$ ) separately. The process of epimerization was monitored by checking crude ${ }^{1} \mathrm{H}$ NMR (after $4 \mathrm{~h}$ and $17 \mathrm{~h}$ ). For both compounds $17 \mathrm{~b}$ and 17c, we did observe the equilibrium process, but along with Diels-Alder product 19. Depending on whether we started with 17b (which itself does not undergo the Diels-Alder) or with 17c (which itself does undergo the Diels-Alder) the ratio differed and the in the latter case reasonable amounts of the Diels-Alder product were formed (due to the requirement of $100{ }^{\circ} \mathrm{C}$ for equilibration) which was not the case in with $17 \mathrm{~b}$ as the starting point. With this outcome we felt that an additional experiment was needed to assure that equilibration of $\mathrm{C}-13$ exclusively occurs prior to the Diels-Alder reaction and that there is no isomerization process being involved afterwards.

\section{Results based on time:}

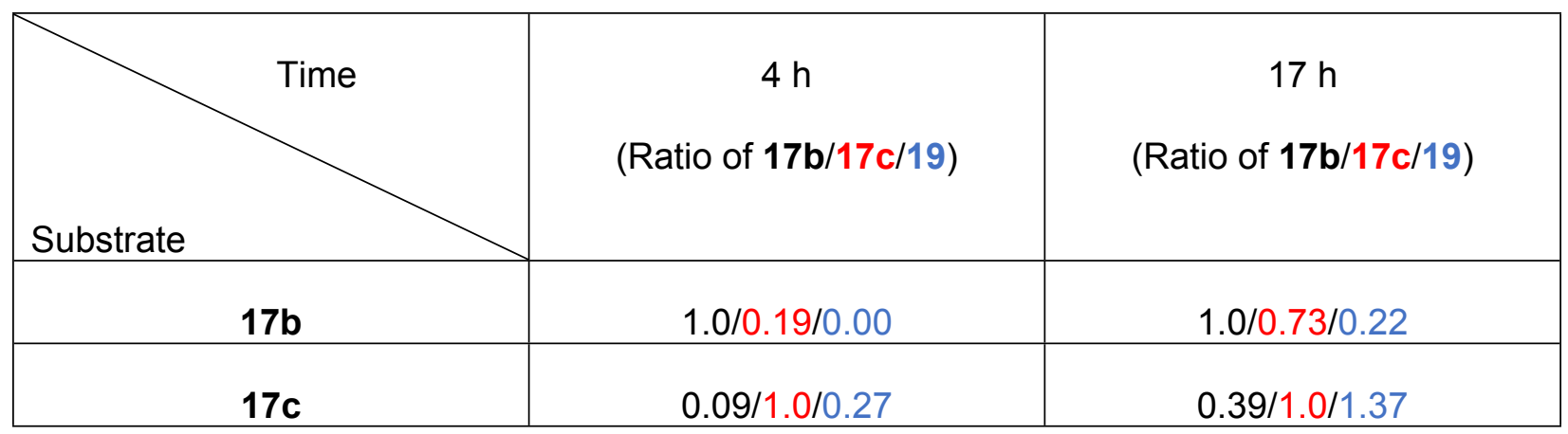



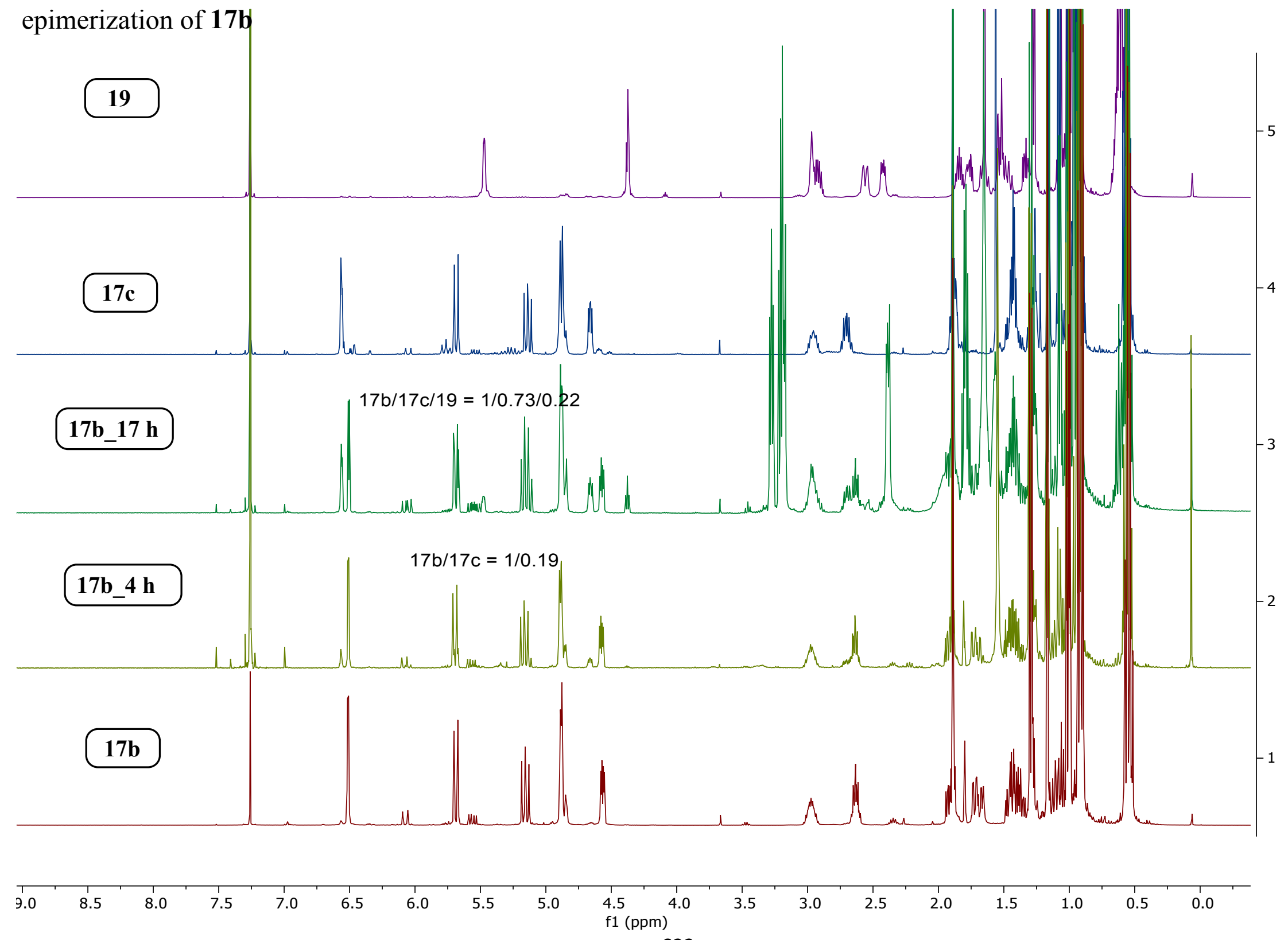

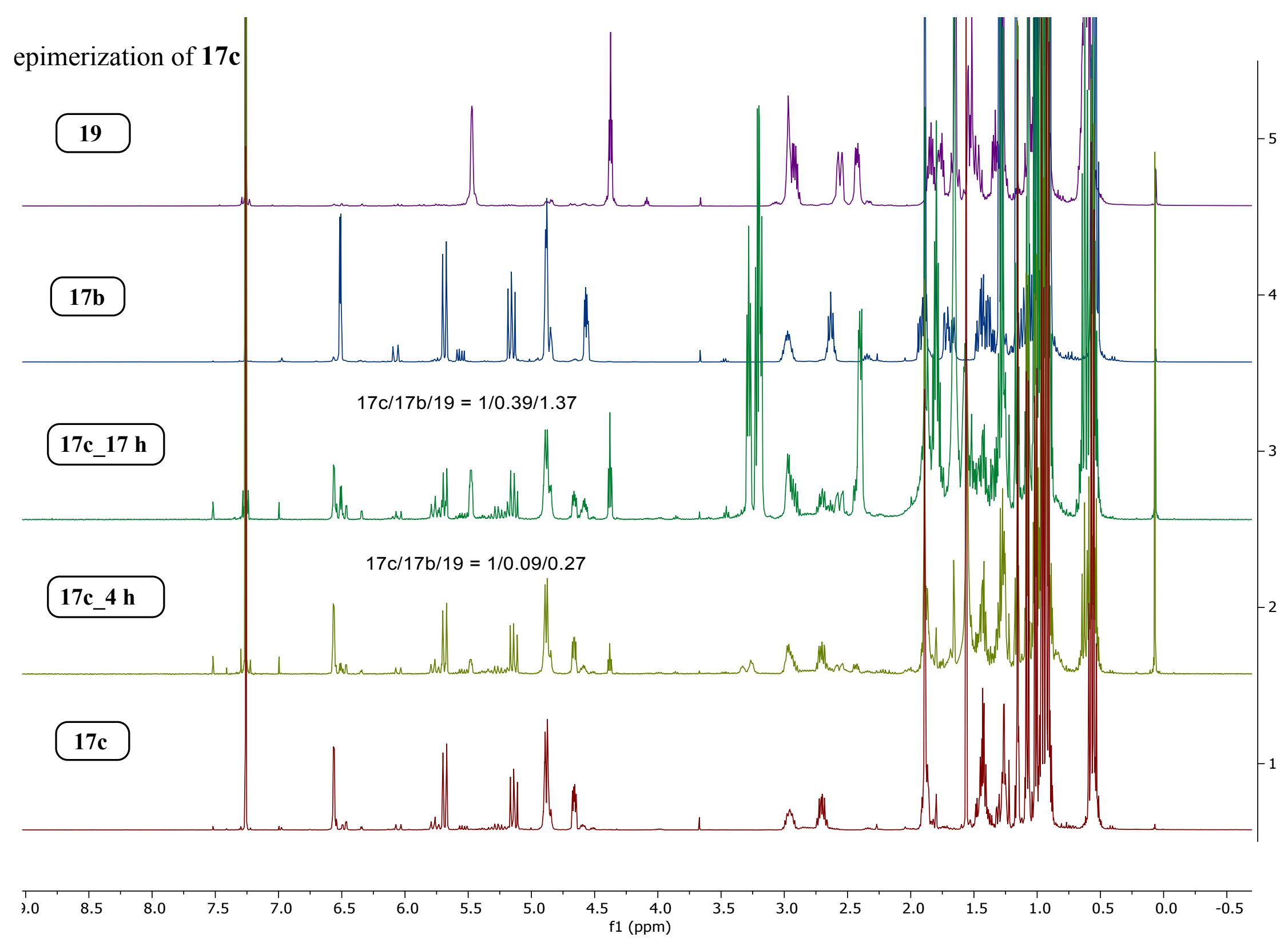


\section{Deuterium Labeling Experiment of epi-19 to deuterated 19 with LDA}

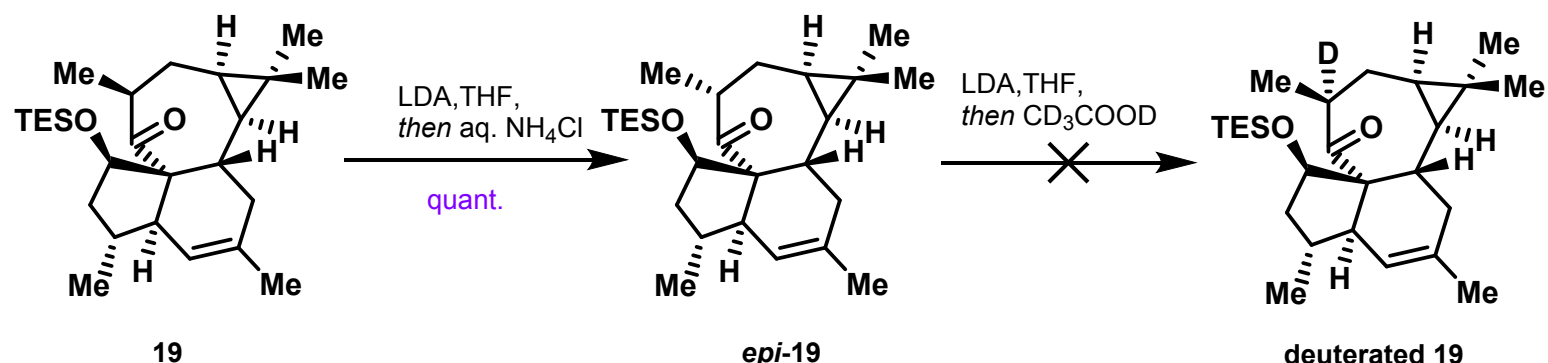

Diisopropylamine (38.0 $\mu \mathrm{L}, 0.267 \mathrm{mmol}, 5.30$ equiv) was dissolved in THF $(0.500 \mathrm{~mL})$ under $\mathrm{N}_{2}$ at $-78{ }^{\circ} \mathrm{C}$. $n$-BuLi $(0.100 \mathrm{~mL}, 0.250 \mathrm{mmol}, 2.5 \mathrm{M}$ in hexane, 5.00 equiv) was added dropwise. The stirring was continued for $20 \mathrm{~min}$ at this temperature. Afterwards, the solution of ketone 19 (21.0 $\mathrm{mg}, 50.0 \mu \mathrm{mol}, 1.00$ equiv) in THF $(0.500 \mathrm{~mL})$ was added dropwise via syringe at $-78{ }^{\circ} \mathrm{C}$. After $50 \mathrm{~min}, 1 \mathrm{~mL}$ saturated aq. $\mathrm{NH}_{4} \mathrm{Cl}$ was added to quench the formed enolate at $-78{ }^{\circ} \mathrm{C}$, and the reaction was allowed to warm to room temperature gradually via removal of cooling bath. Then the reaction was diluted with $\mathrm{Et}_{2} \mathrm{O}$, extracted $\left(\mathrm{Et}_{2} \mathrm{O} \times 3\right)$. The combined organic layers were dried over $\mathrm{MgSO}_{4}$, filtered and evaporated under reduced pressure. The remaining residue was purified by column chromatography on silica gel (PE/EA $=40 / 1)$, yielding epi-19 (21.0 mg, quant.) as a colourless oil.

Then epi-19 (21.0 mg, $50.0 \mu \mathrm{mol}, 1.00$ equiv) was re-subjected to the above identical procedure. But the reaction was quenched by deuterated acetic acid $(30.0 \mu \mathrm{L}, 0.524 \mathrm{mmol}, 10.5$ equiv) at $78{ }^{\circ} \mathrm{C}$ and warmed to room temperature gradually via removal of cooling bath. Then the reaction was diluted with saturated aq. $\mathrm{NaHCO}_{3}$, and extracted with $\mathrm{Et}_{2} \mathrm{O}$ three times. The combined organic layers were dried over $\mathrm{MgSO}_{4}$, filtered and evaporated under reduced pressure. The remaining residue was purified by column chromatography on silica gel $(P E / E A=40 / 1)$, only yielding epi-19 back, which means that LDA fails to deprotonate epi-19.

$\mathbf{R}_{\mathrm{f}}=0.60(\mathrm{PE} / \mathrm{EA}=20 / 1, \mathrm{CAM})$;

${ }^{1} \mathrm{H}$ NMR $\left(400 \mathrm{MHz}, \mathrm{CDCl}_{3}\right) \delta 5.45$ (ddq, $\left.J=4.3,2.9,1.4 \mathrm{~Hz}, 1 \mathrm{H}\right), 4.09(\mathrm{t}, J=5.94 \mathrm{~Hz}, 1 \mathrm{H}), 3.13-$ $3.02(\mathrm{~m}, 1 \mathrm{H}), 2.98(\mathrm{~s}, 1 \mathrm{H}), 2.59$ (ddtd, $J=16.1,5.2,2.4,1.3 \mathrm{~Hz}, 1 \mathrm{H}), 2.34$ (ddd, $J=11.5,5.4,1.9$ $\mathrm{Hz}, 1 \mathrm{H}), 1.87$ (tq, $J=6.1,4.0 \mathrm{~Hz}, 1 \mathrm{H}), 1.79$ (ddd, $J=14.4,8.5,4.9 \mathrm{~Hz}, 1 \mathrm{H}), 1.72-1.64(\mathrm{~m}, 4 \mathrm{H})$, $1.63-1.58(\mathrm{~m}, 1 \mathrm{H}), 1.57-1.49(\mathrm{~m}, 1 \mathrm{H}), 1.37(\mathrm{dtd}, J=12.0,5.6,0.6 \mathrm{~Hz}, 1 \mathrm{H}), 1.05(\mathrm{~d}, J=6.5 \mathrm{~Hz}$, $3 \mathrm{H}), 1.02(\mathrm{~s}, 3 \mathrm{H}), 0.99-0.94(\mathrm{~m}, 12 \mathrm{H}), 0.94(\mathrm{~s}, 3 \mathrm{H}), 0.65-0.56(\mathrm{~m}, 7 \mathrm{H}), 0.52(\mathrm{td}, J=9.3,5.0 \mathrm{~Hz}$, 1H) ppm; 
${ }^{13} \mathrm{C}$ NMR $\left(100 \mathrm{MHz}, \mathrm{CDCl}_{3}\right) \delta 215.0,129.7,124.9,76.7,65.6,42.6,41.8,39.8,39.4,34.8,32.0$, 31.3, 29.7, 29.2, 24.6, 22.9, 19.8, 18.4, 18.3, 14.8, 7.0, 5.1 ppm;

HRMS (ESI): $\mathrm{m} / \mathrm{z}$ calcd for $\mathrm{C}_{26} \mathrm{H}_{45} \mathrm{O}_{2} \mathrm{Si}^{+}[M+\mathrm{H}]^{+}:$: 417.3183, found: 417.3180;

IR (neat sample): 2954, 2876, 1711, 1456, 1414, 1375, 1239, 1191, 1163, 1101, 1052, 1003, 976 , $957,910,844,828,790,725 \mathrm{~cm}^{-1}$;

$[\alpha]_{D} 5:-114.5\left(c 0.65, \mathrm{CHCl}_{3}\right)$.

Explanation for the failed deuterium incorporation (yellow lines below):

The alignment of the proton at C-13 and the carbonyl group in 19 is perpendicular (or $\sigma_{(\mathrm{C}-\mathrm{H})}$ and $\pi_{(\mathrm{C}-\mathrm{O})}$ are parallel).

Therefore the $\mathrm{C}-\mathrm{H}$ acidity is fully maintained. Hence deprotonation is possible.

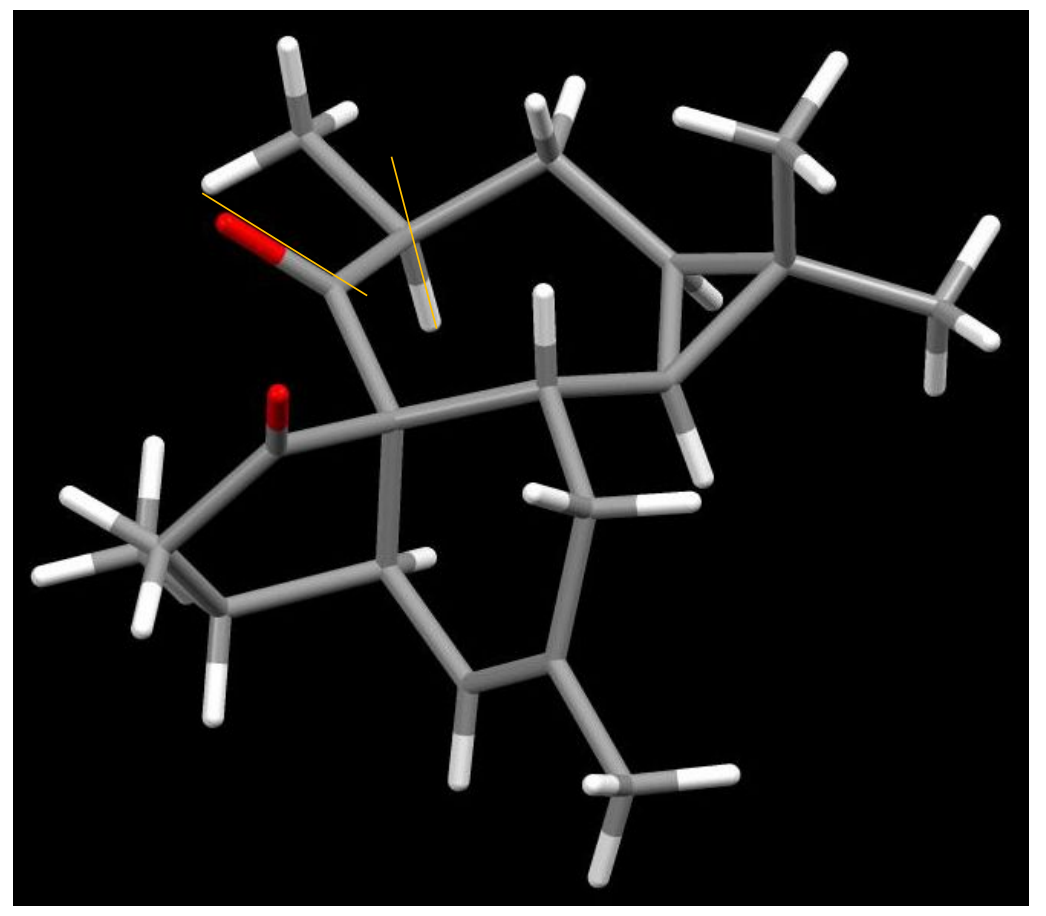


The alignment of the proton at C-13 and the carbonyl group in epi-19 is "in-plane". Therefore the proton is no longer acidic. Hence no deprotonation takes place

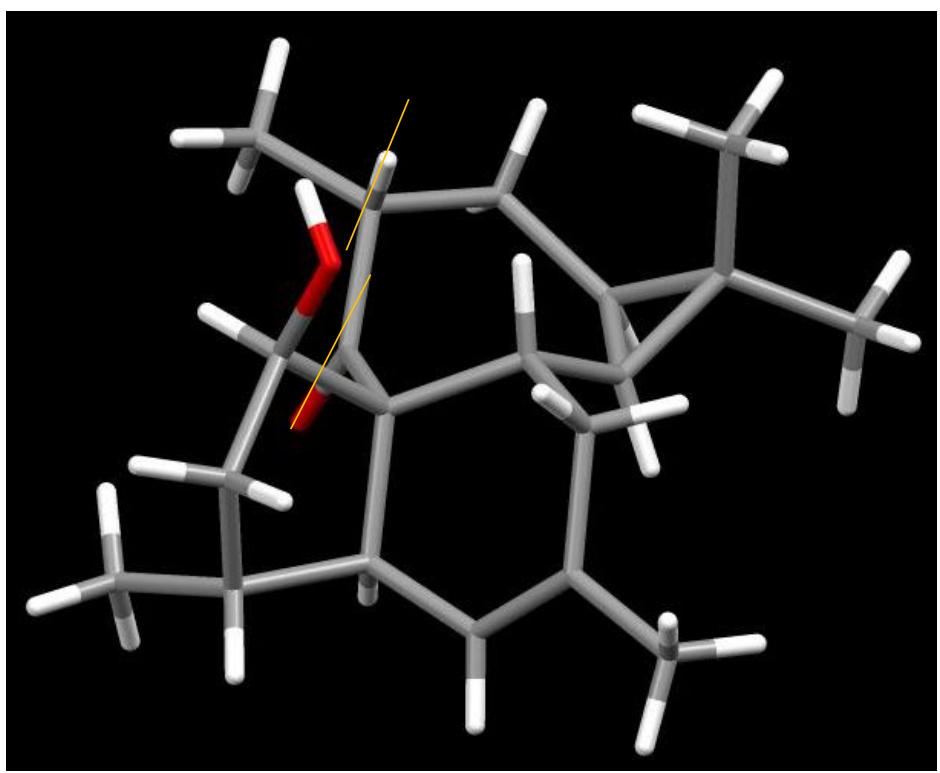


4. ${ }^{1} \mathrm{H}-$ and ${ }^{13} \mathrm{C}-\mathrm{NMR}$ Spectra
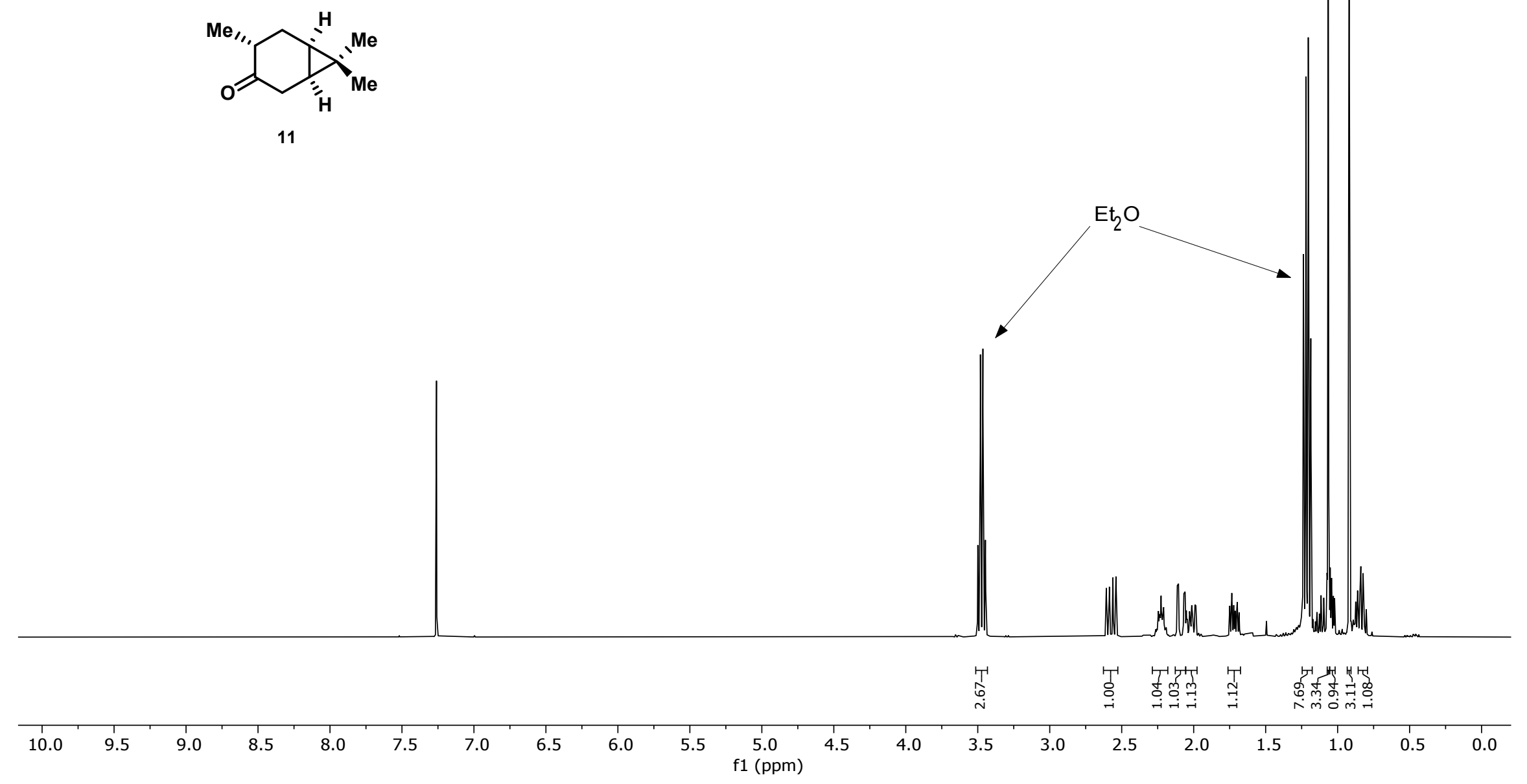

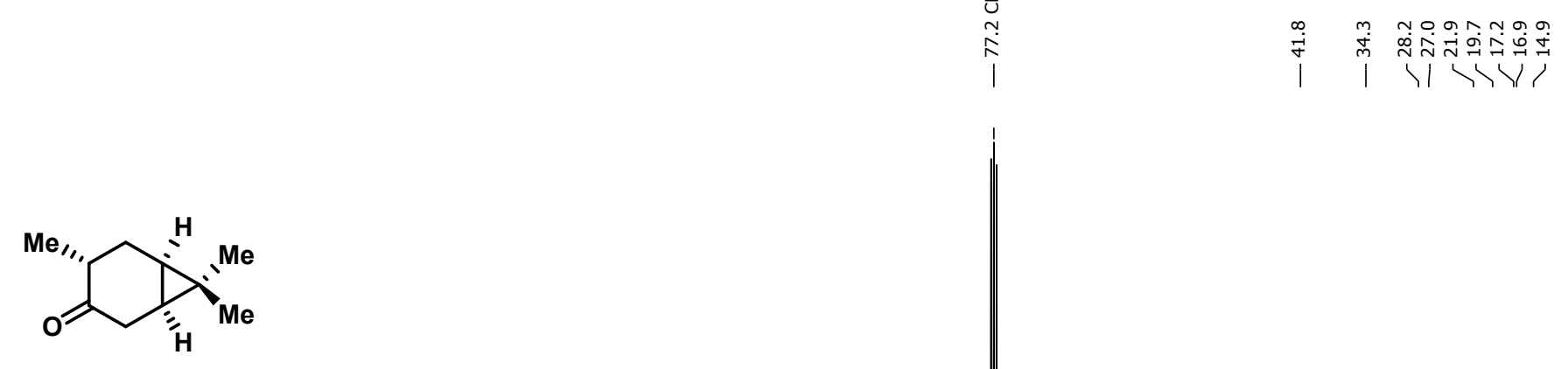

11

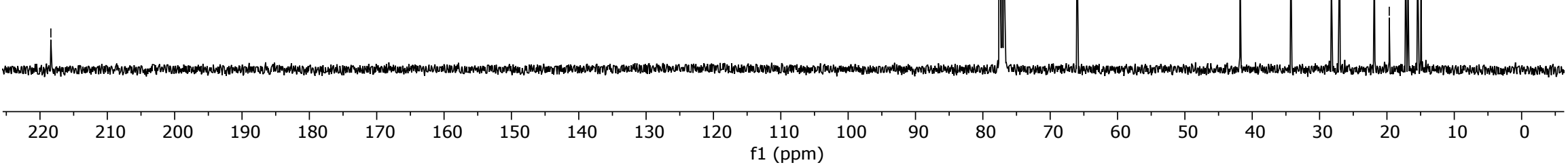



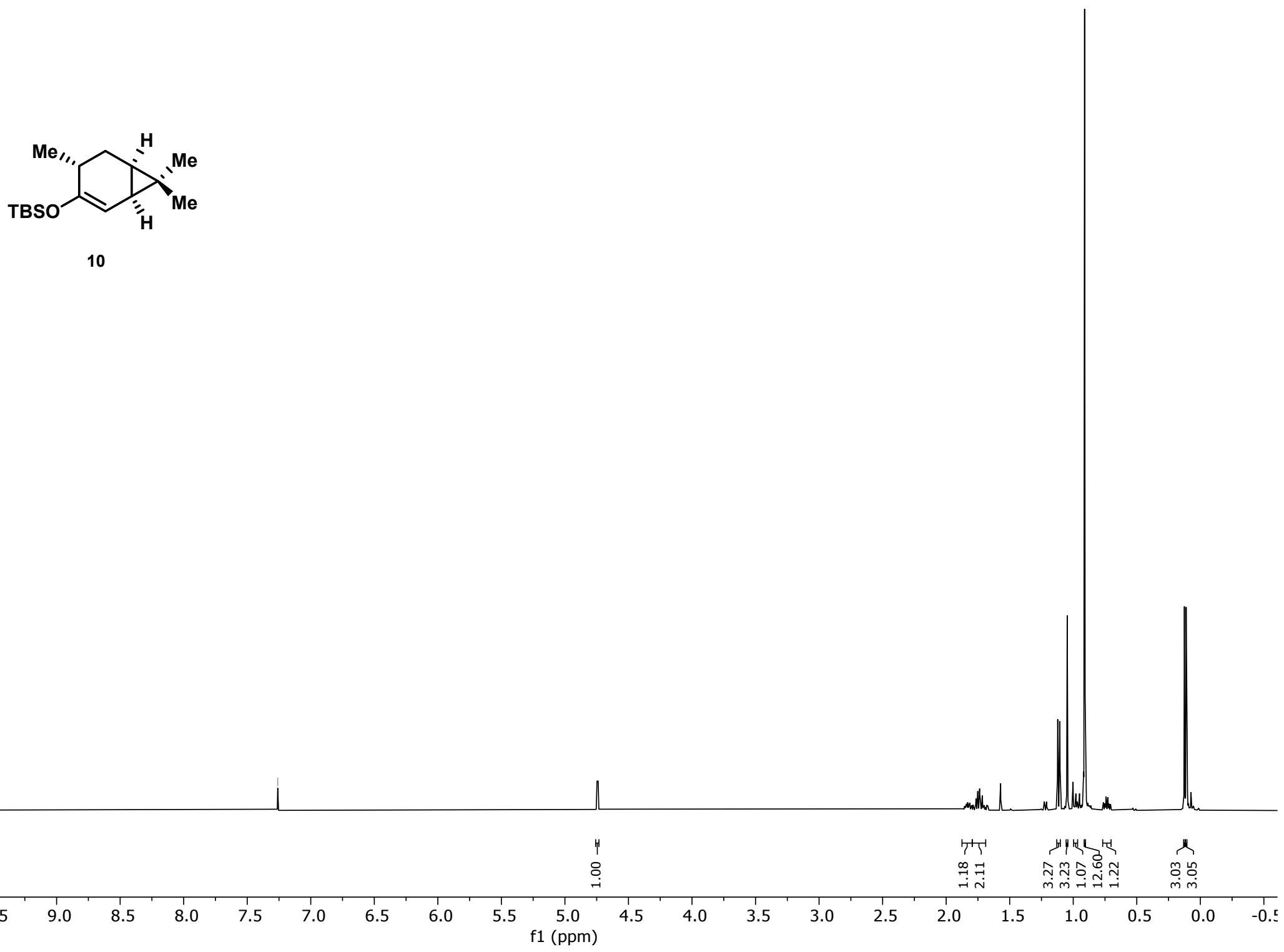


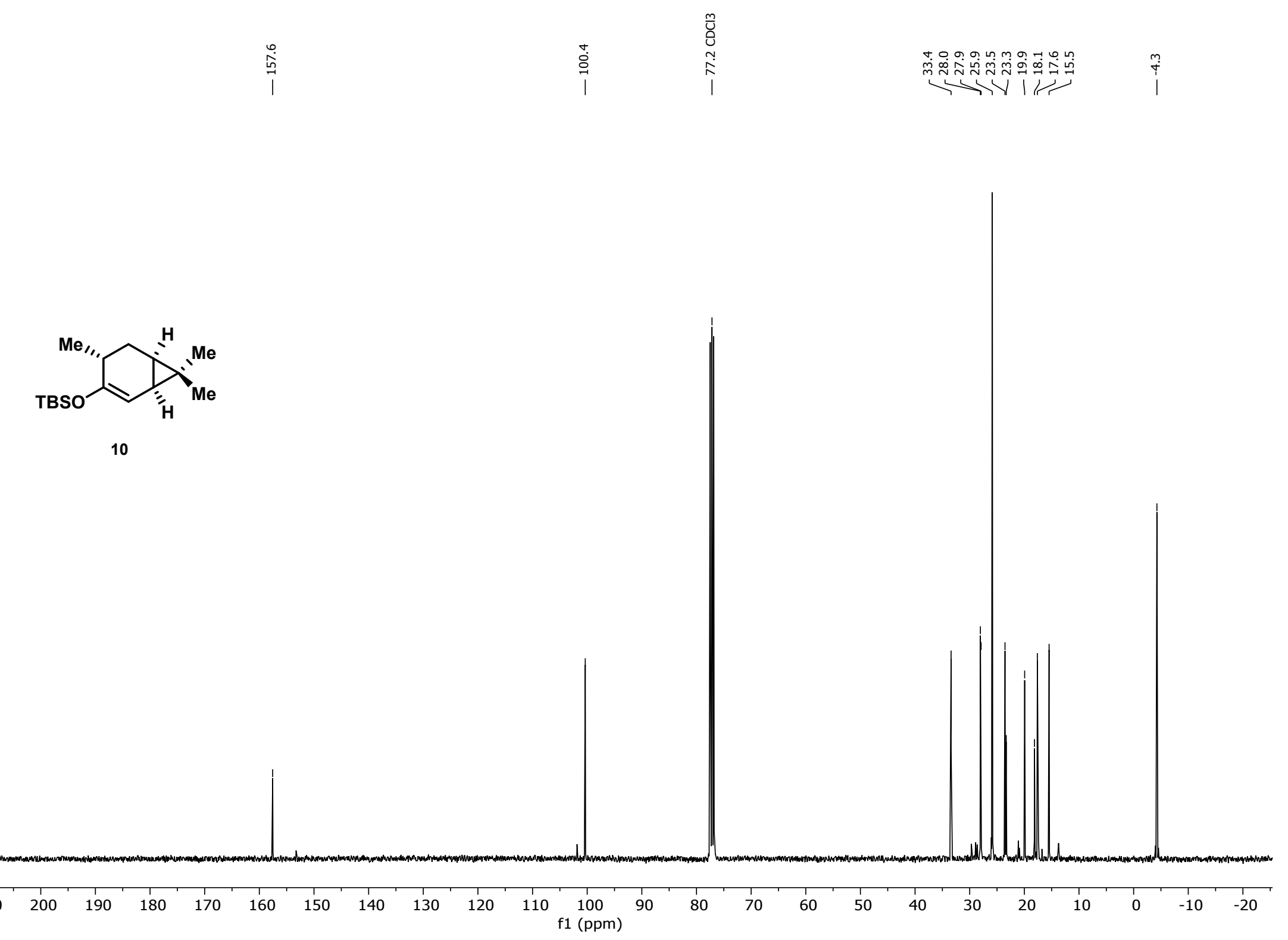



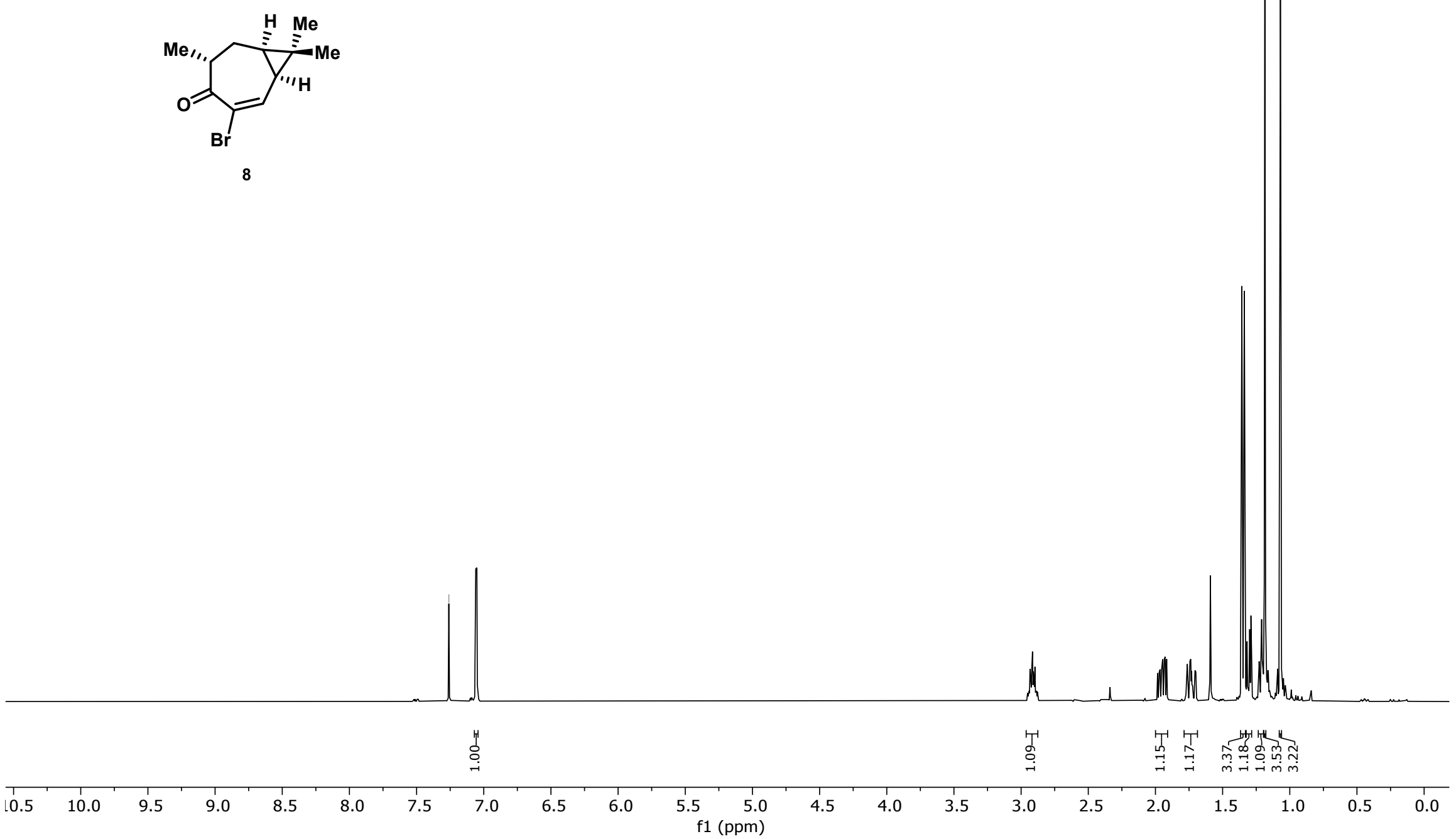
$\mathrm{H}$ Me

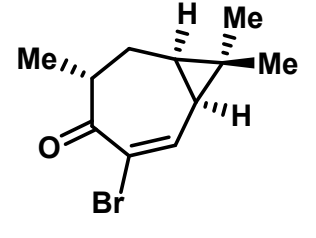

8

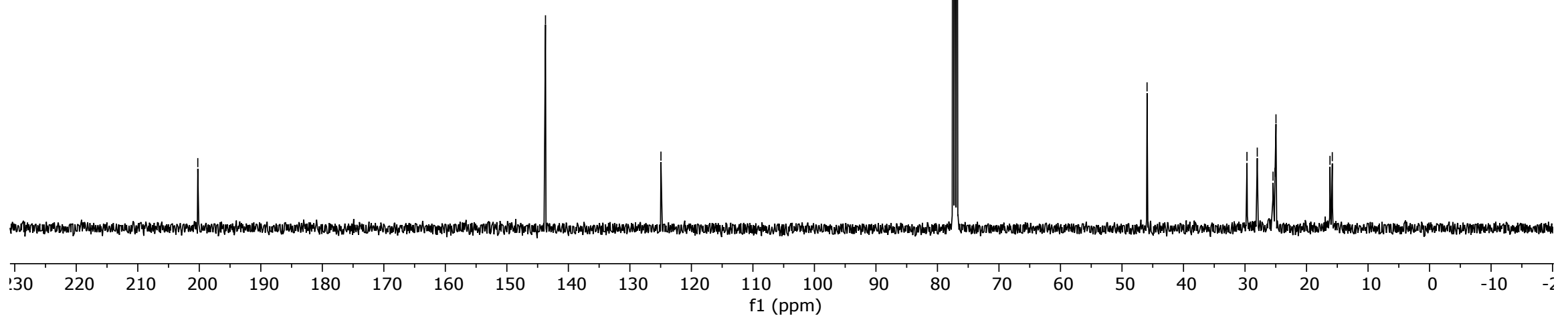




\section{$\overbrace{S}^{\mathrm{Me}}$}

15

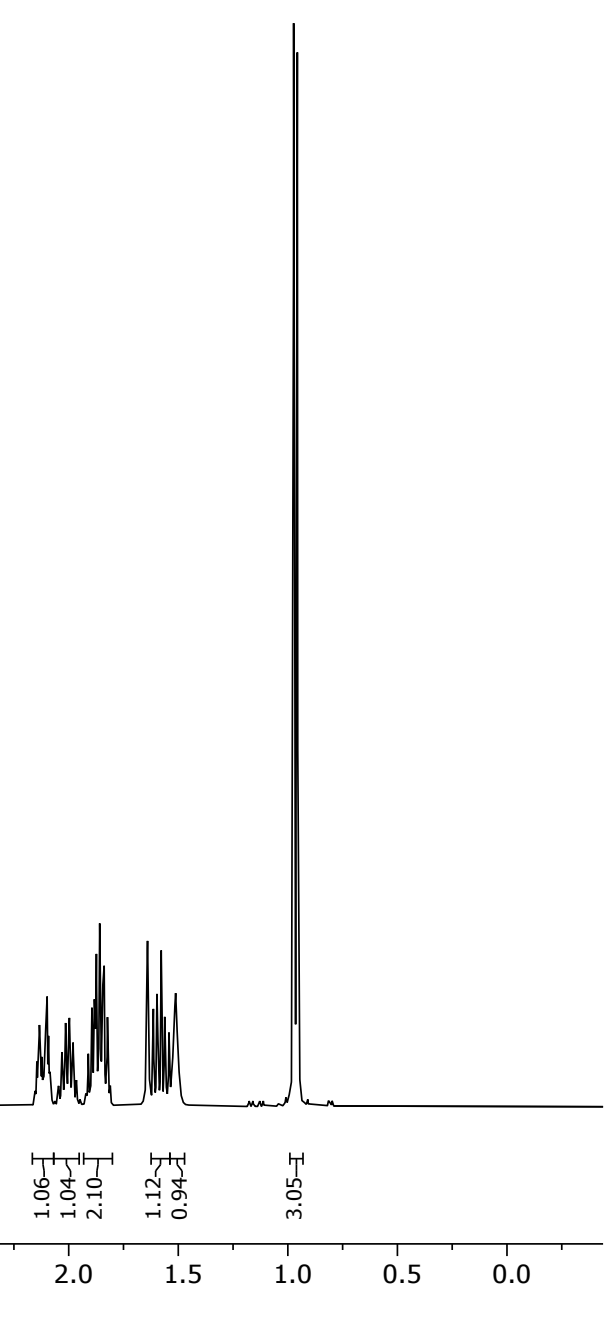




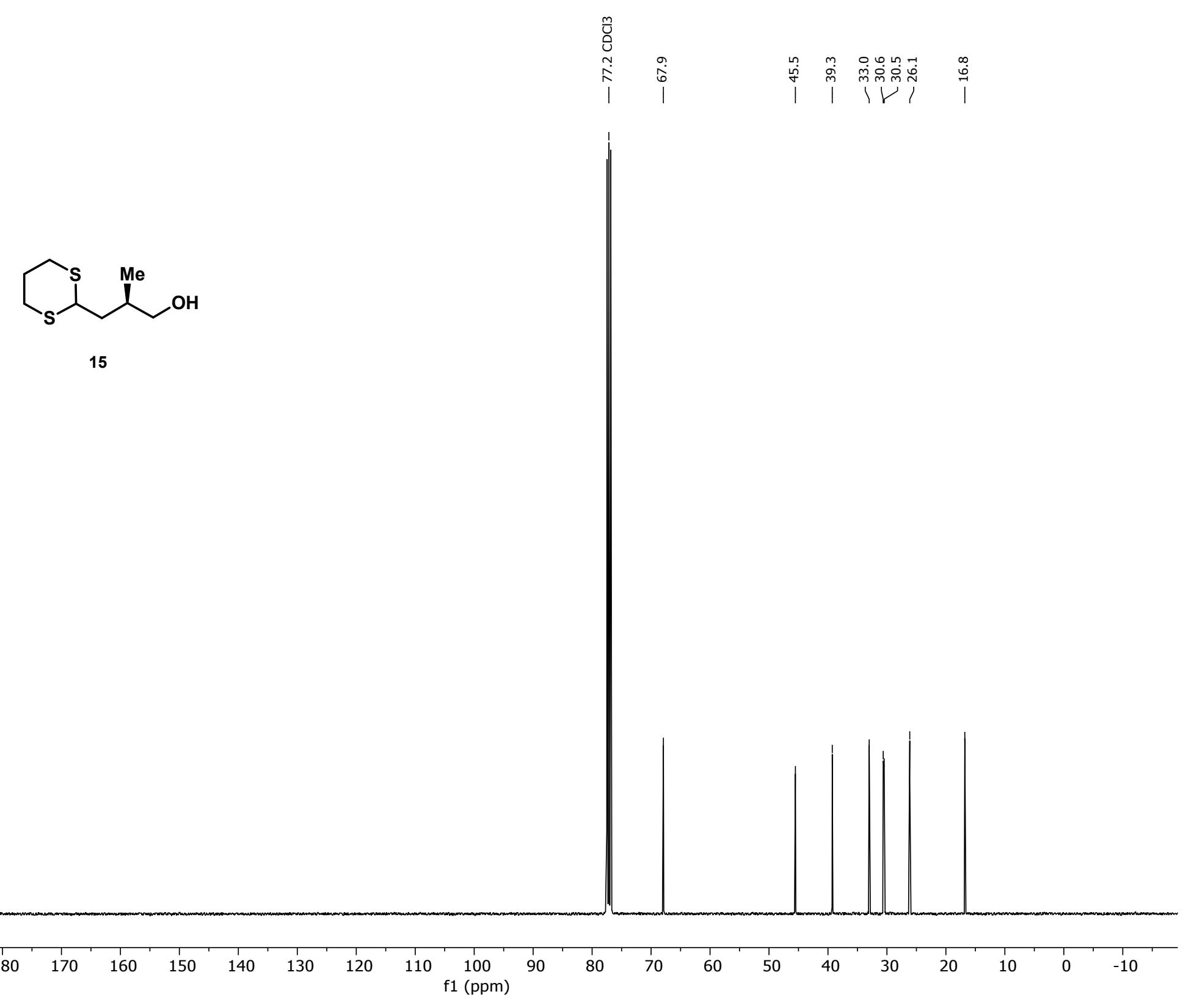




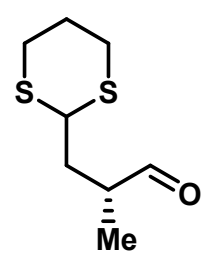

13

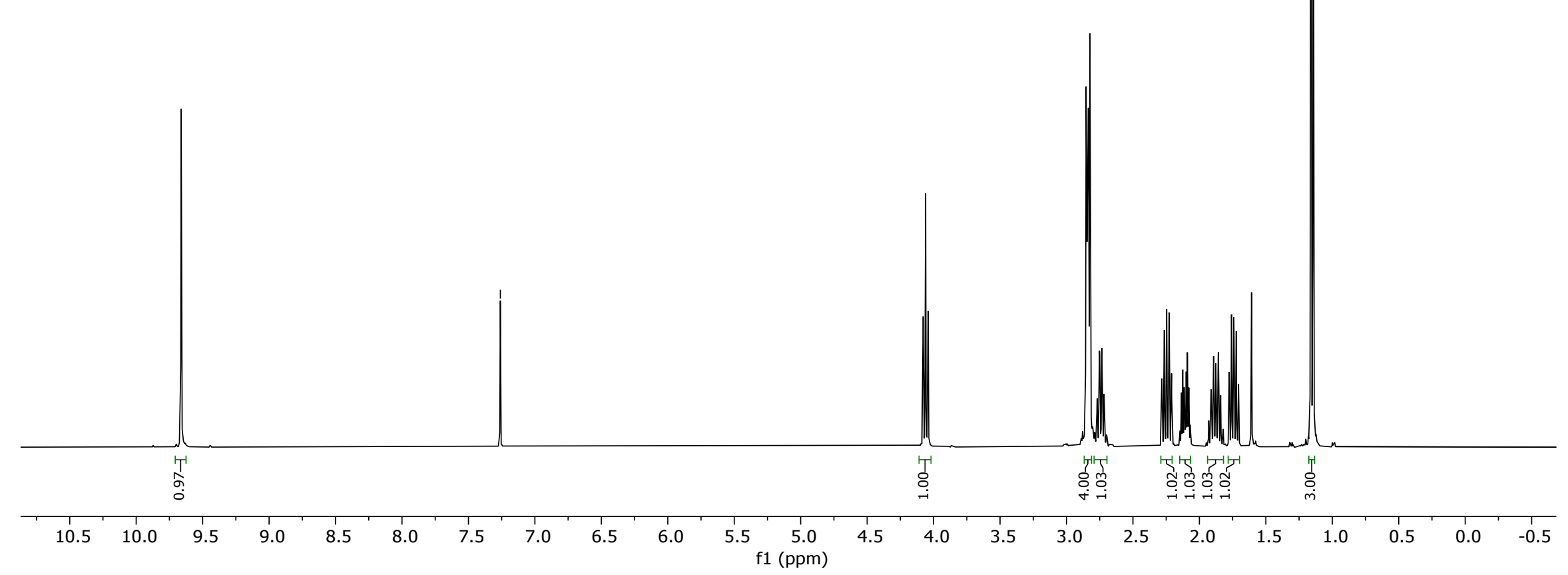




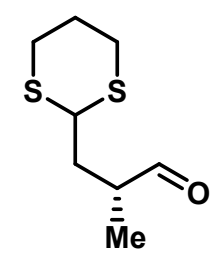

13

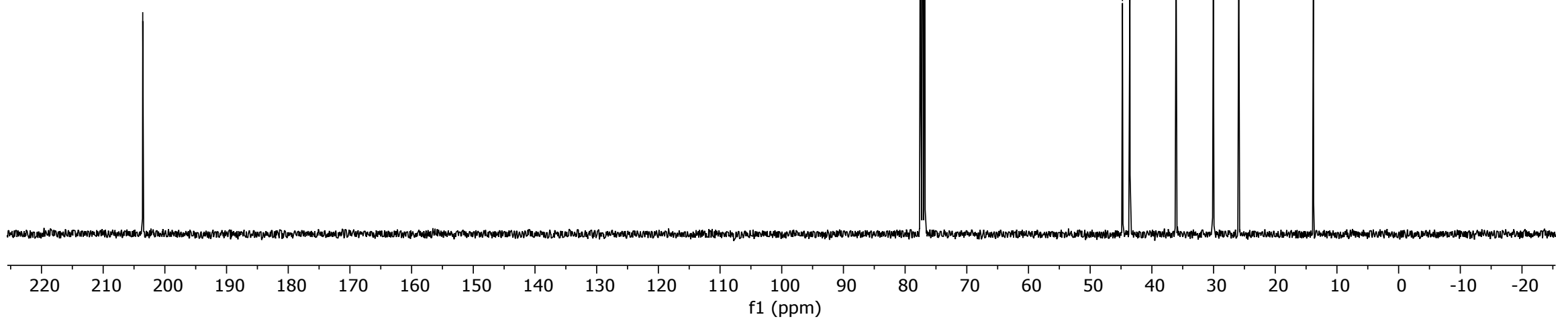




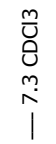

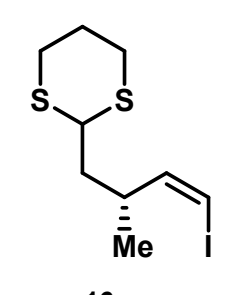

16

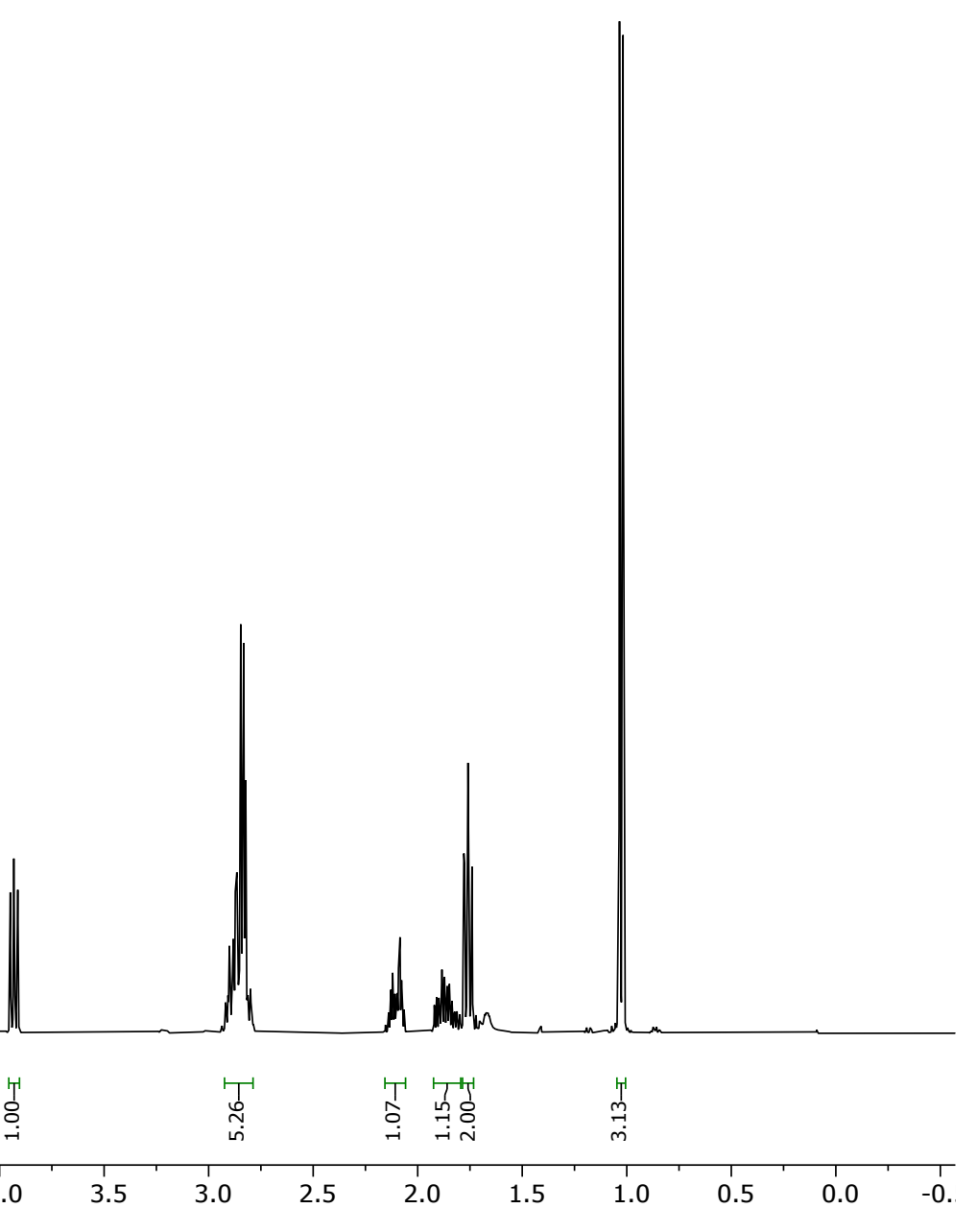




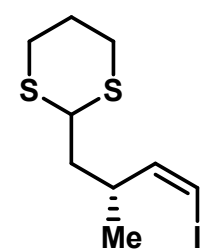

$11<V \mid$

16

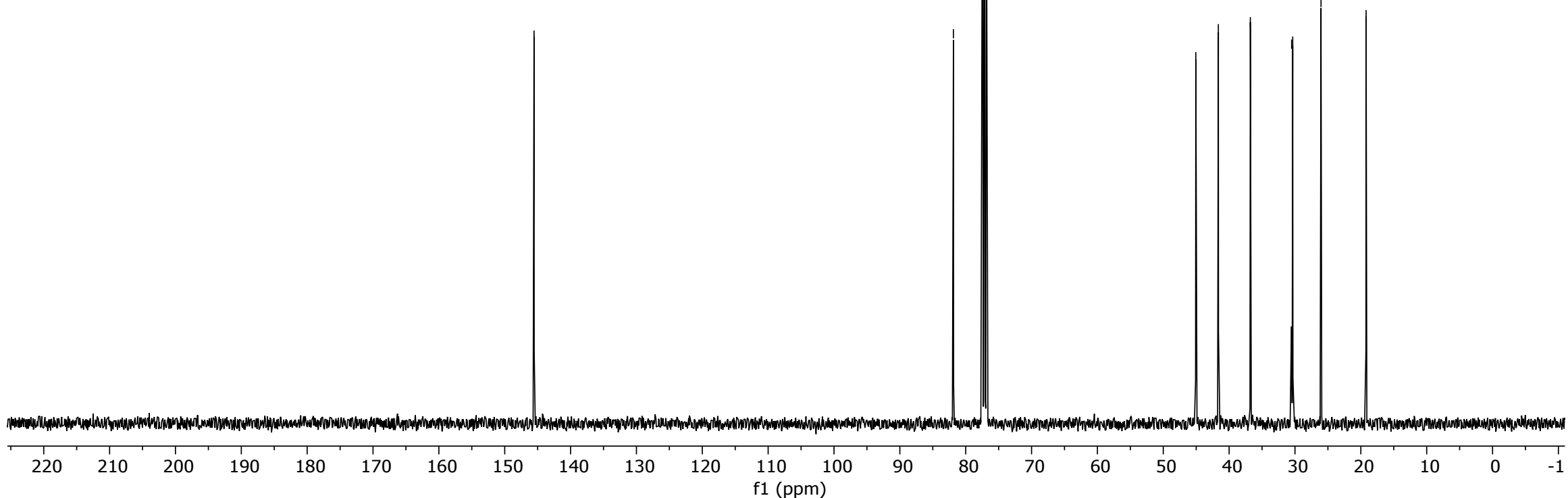



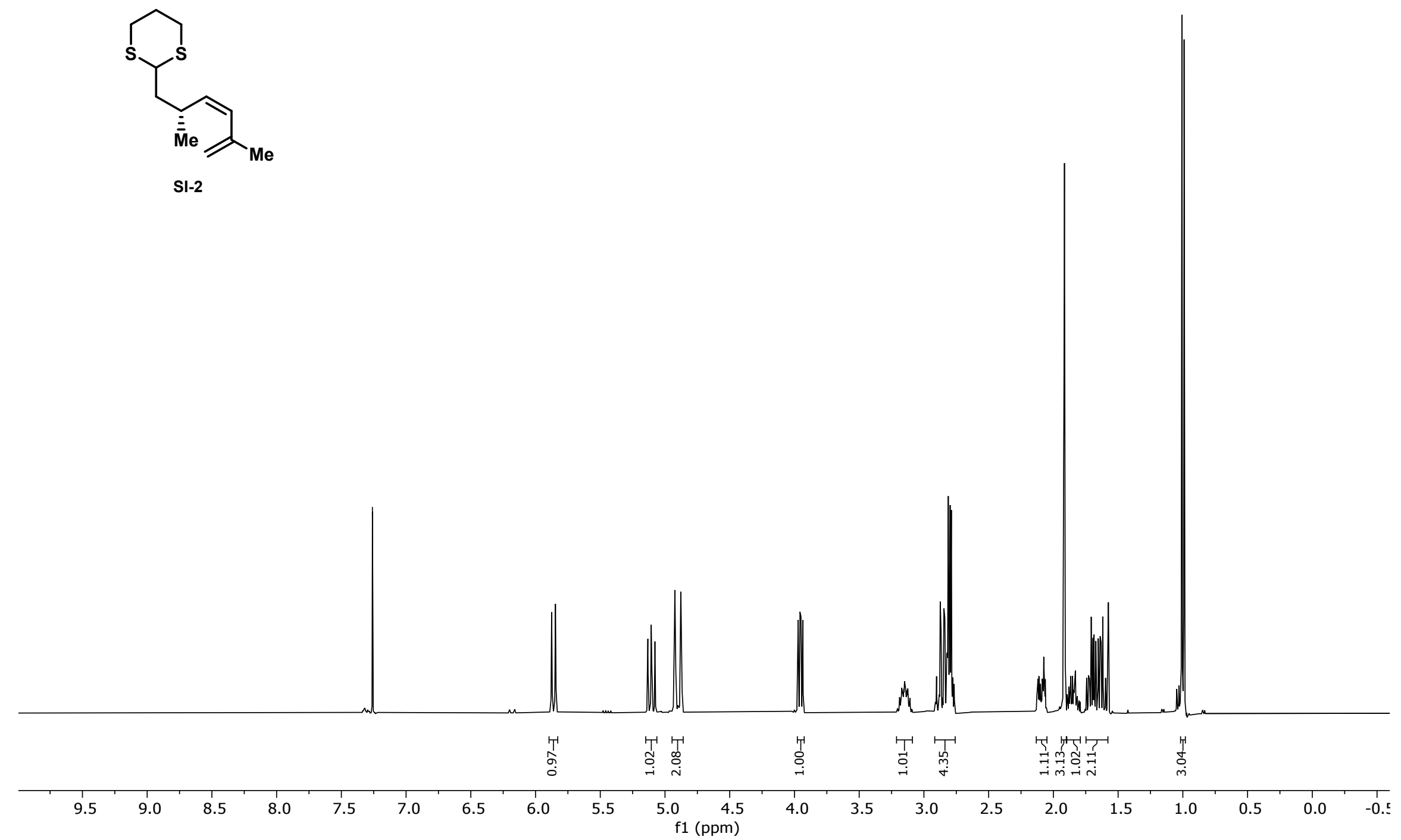


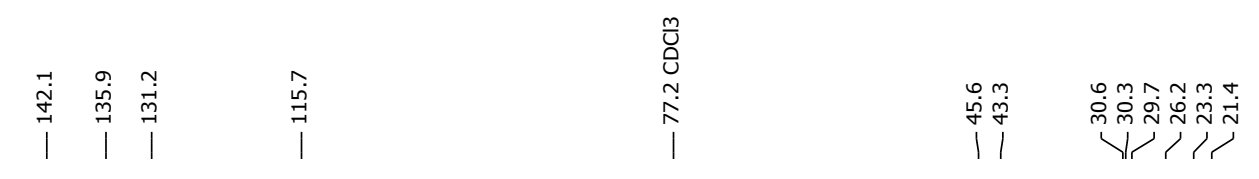

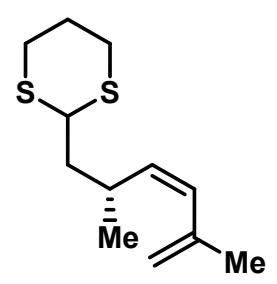

SI-2
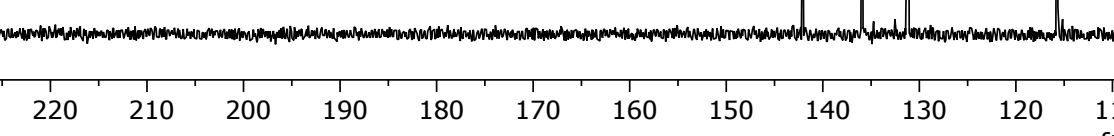

$110 \quad 100$

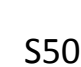



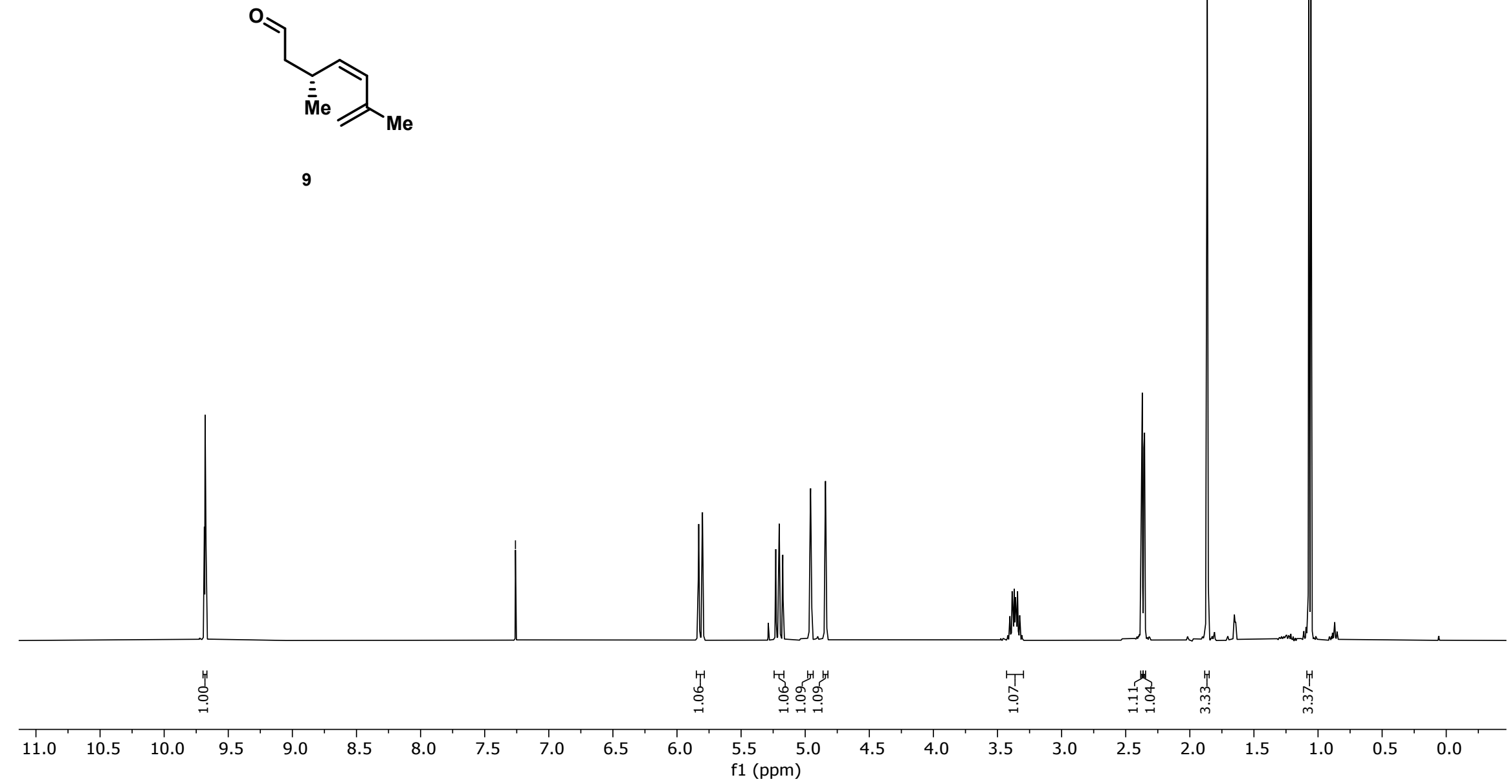

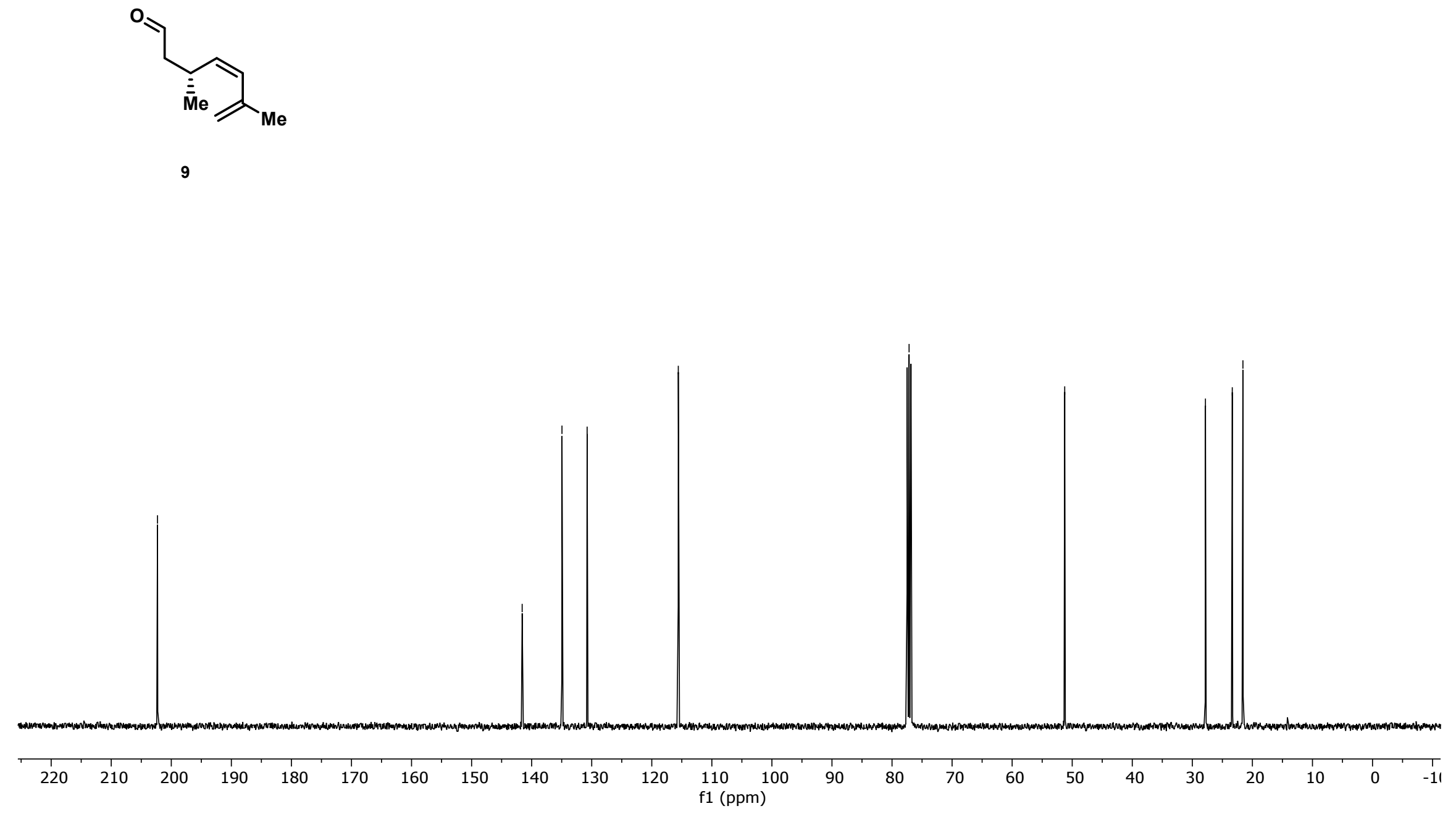


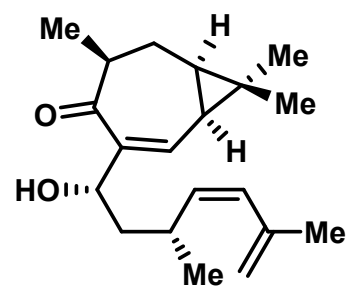

$7 a$

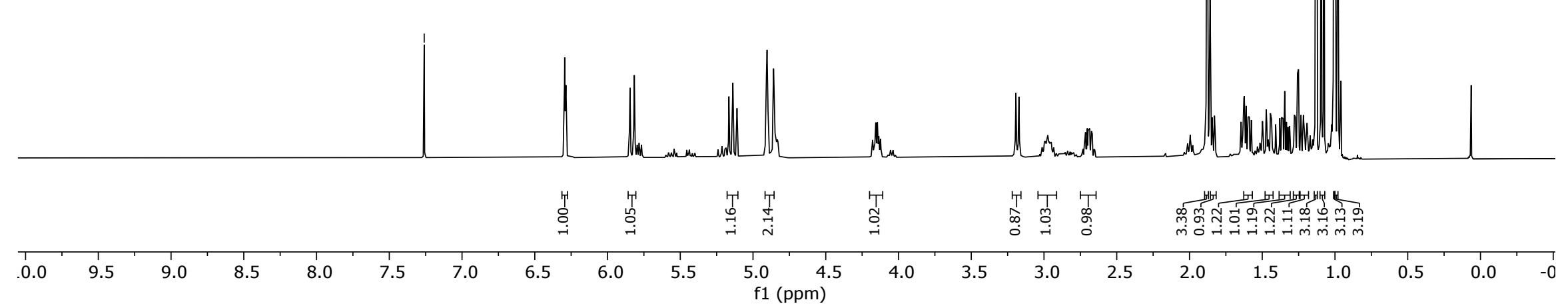




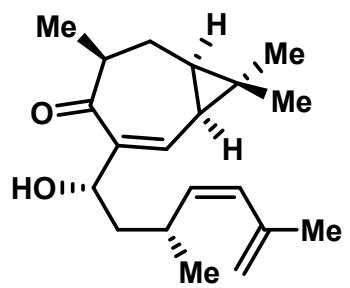

$7 a$

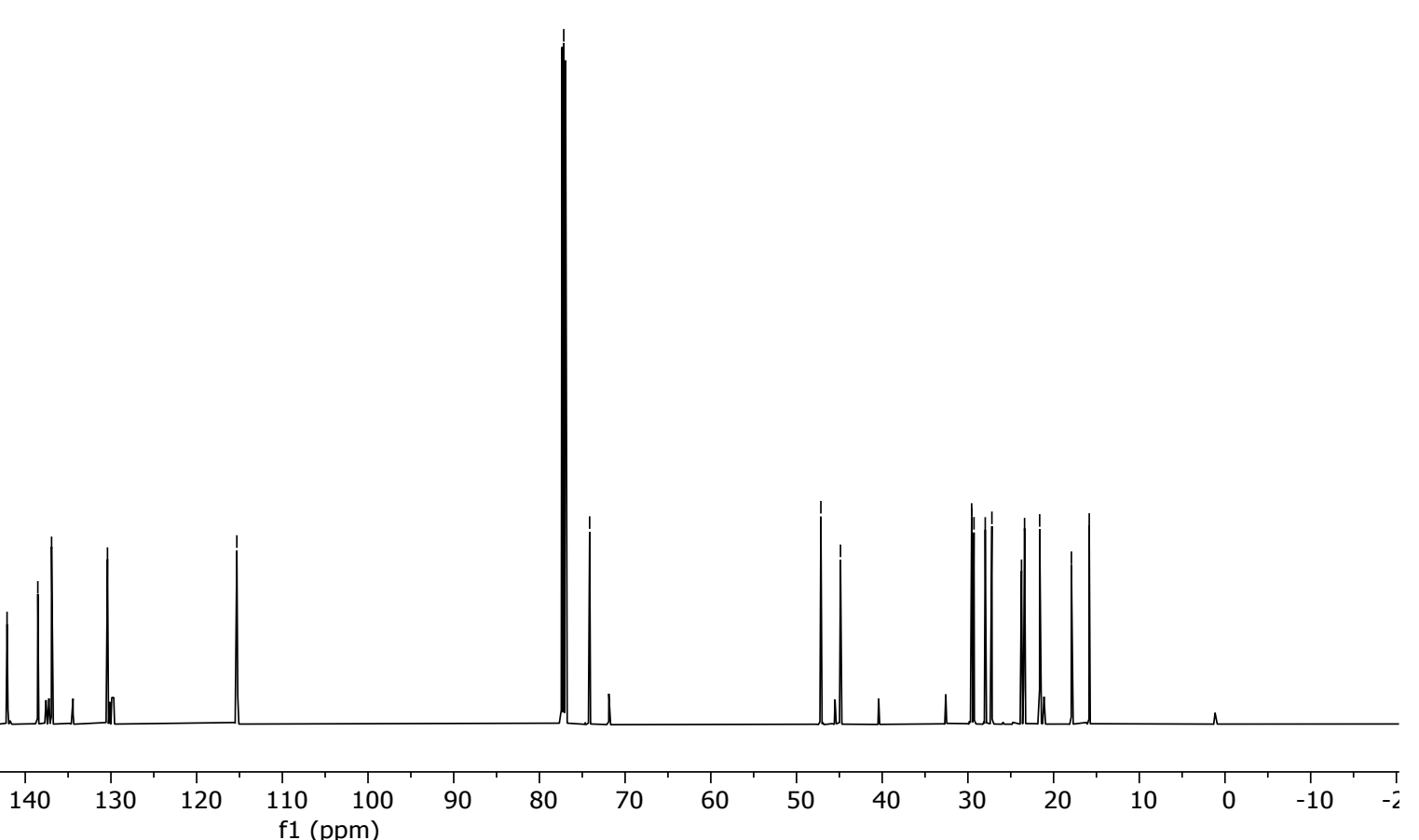




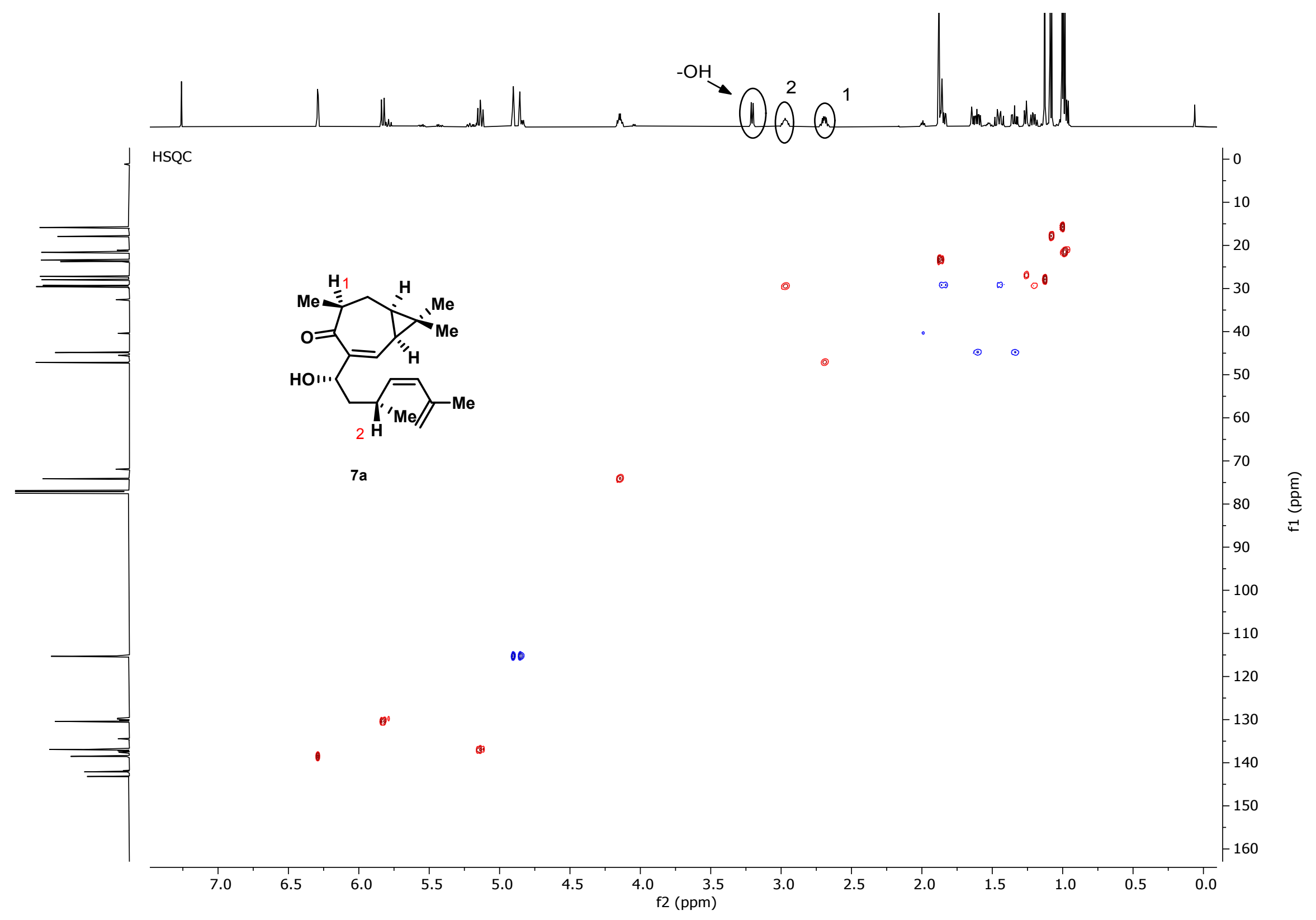




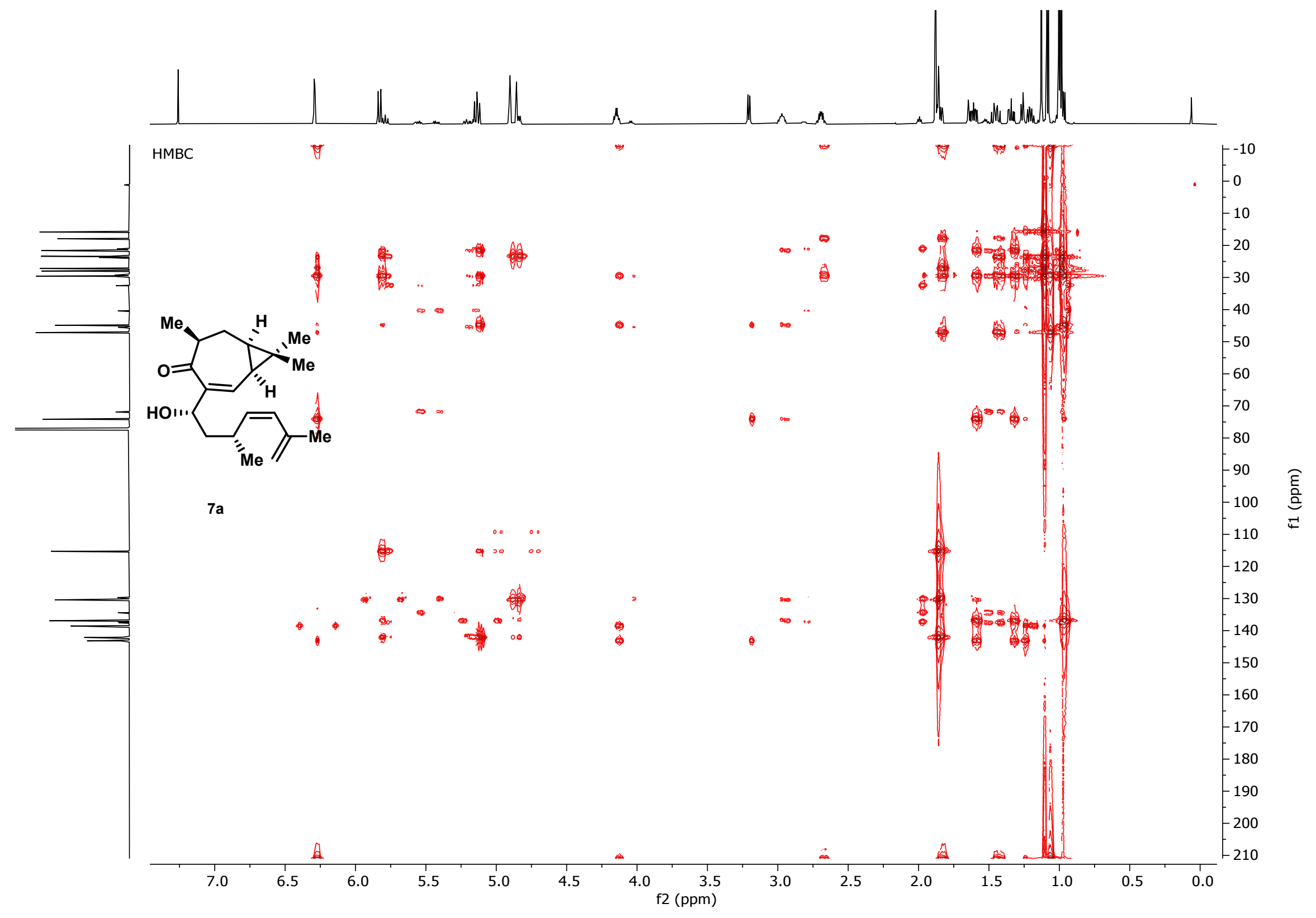




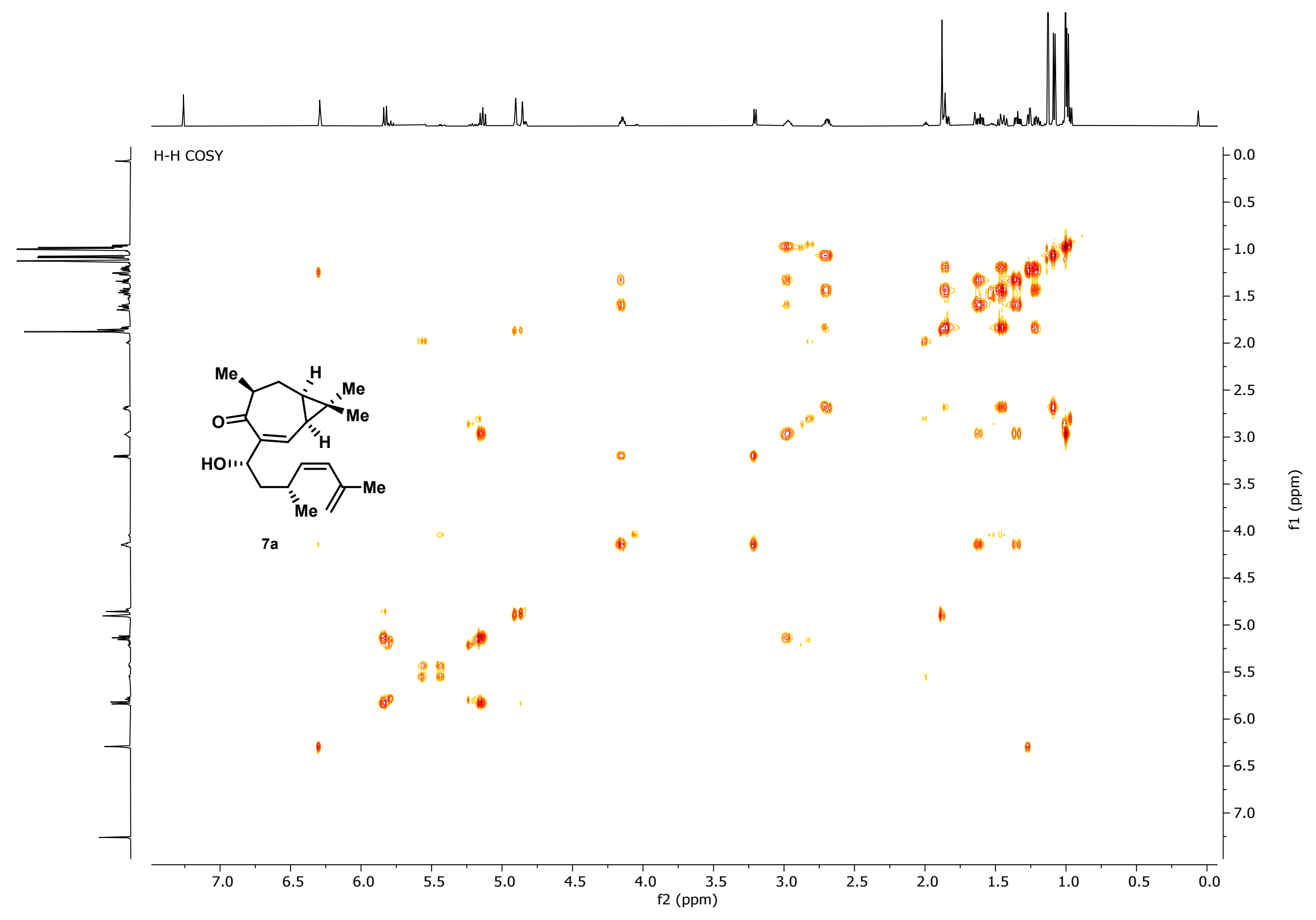




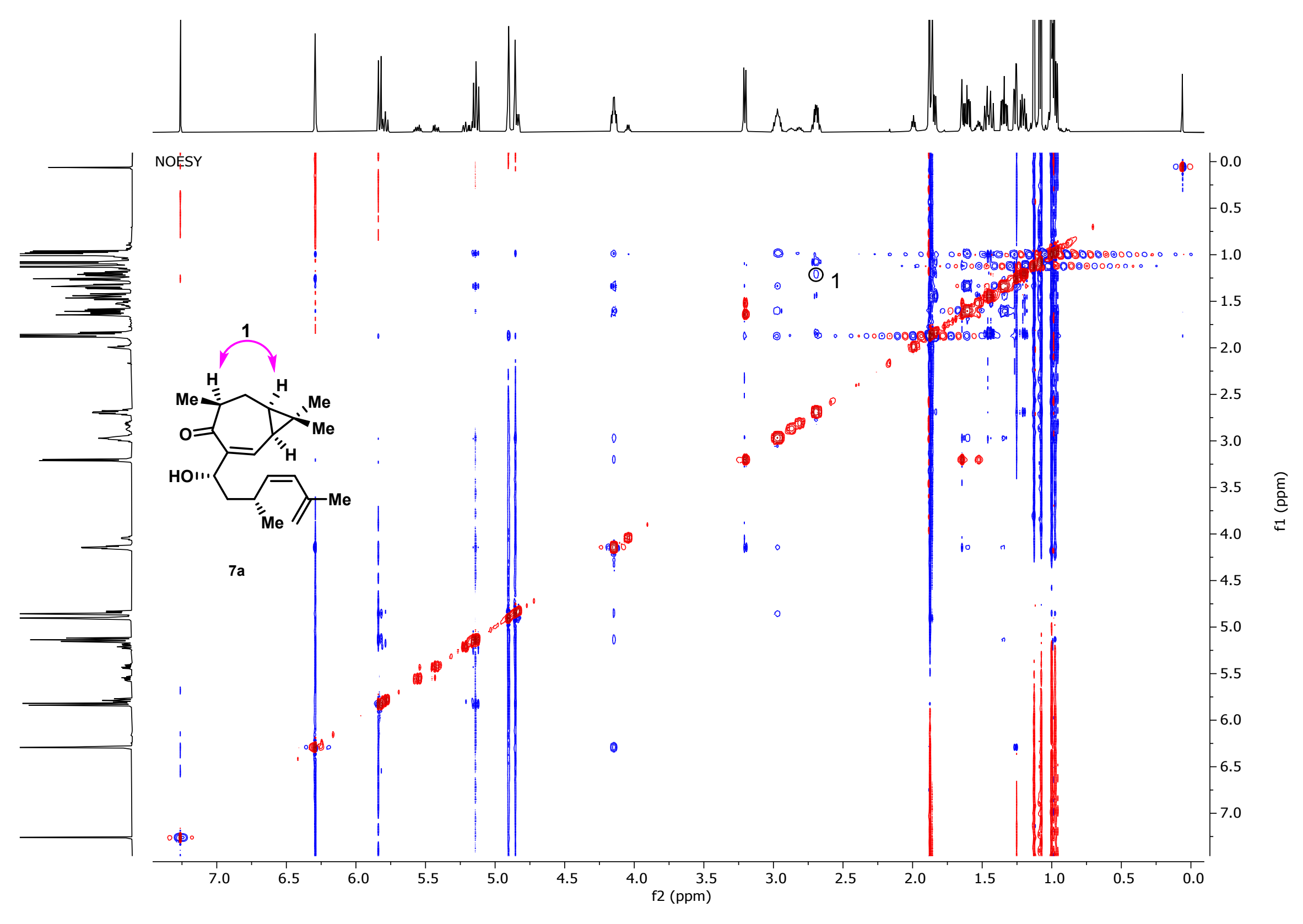




\section{$\frac{m}{0}$
0
0}

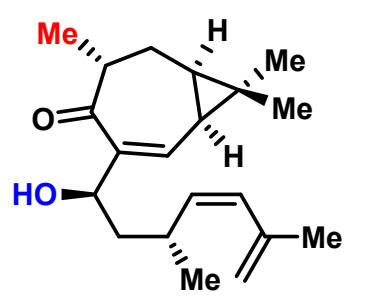

$7 b$

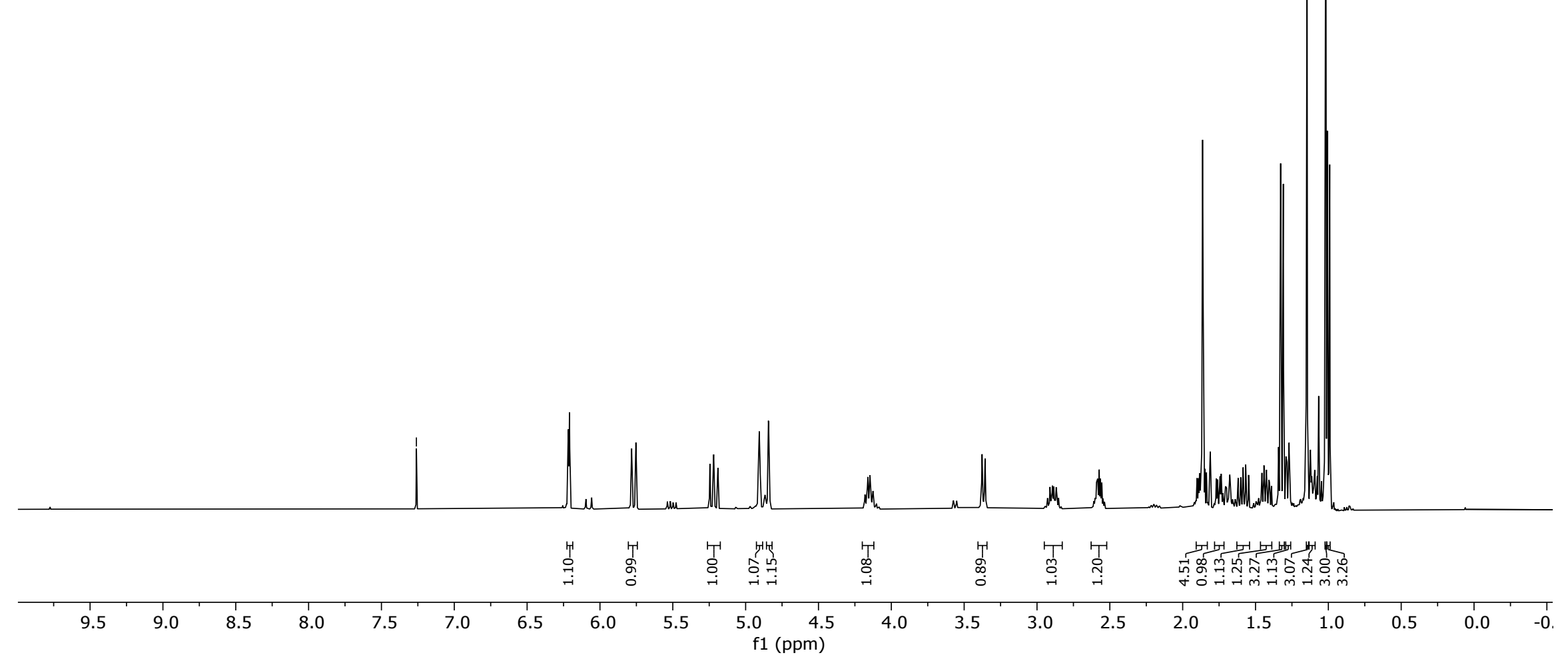




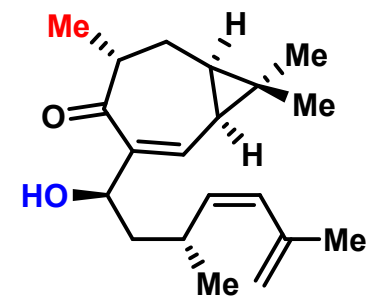

$7 b$

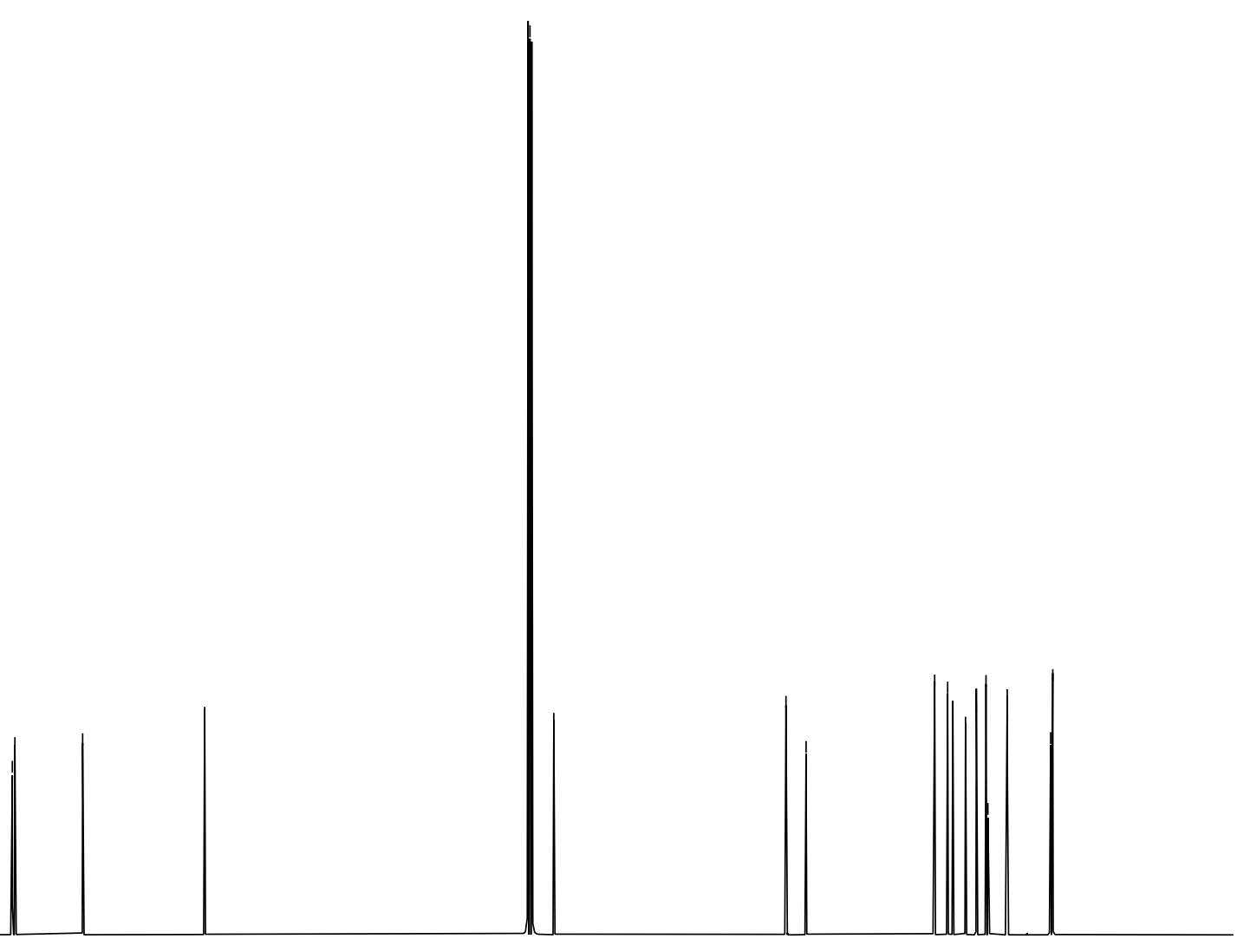




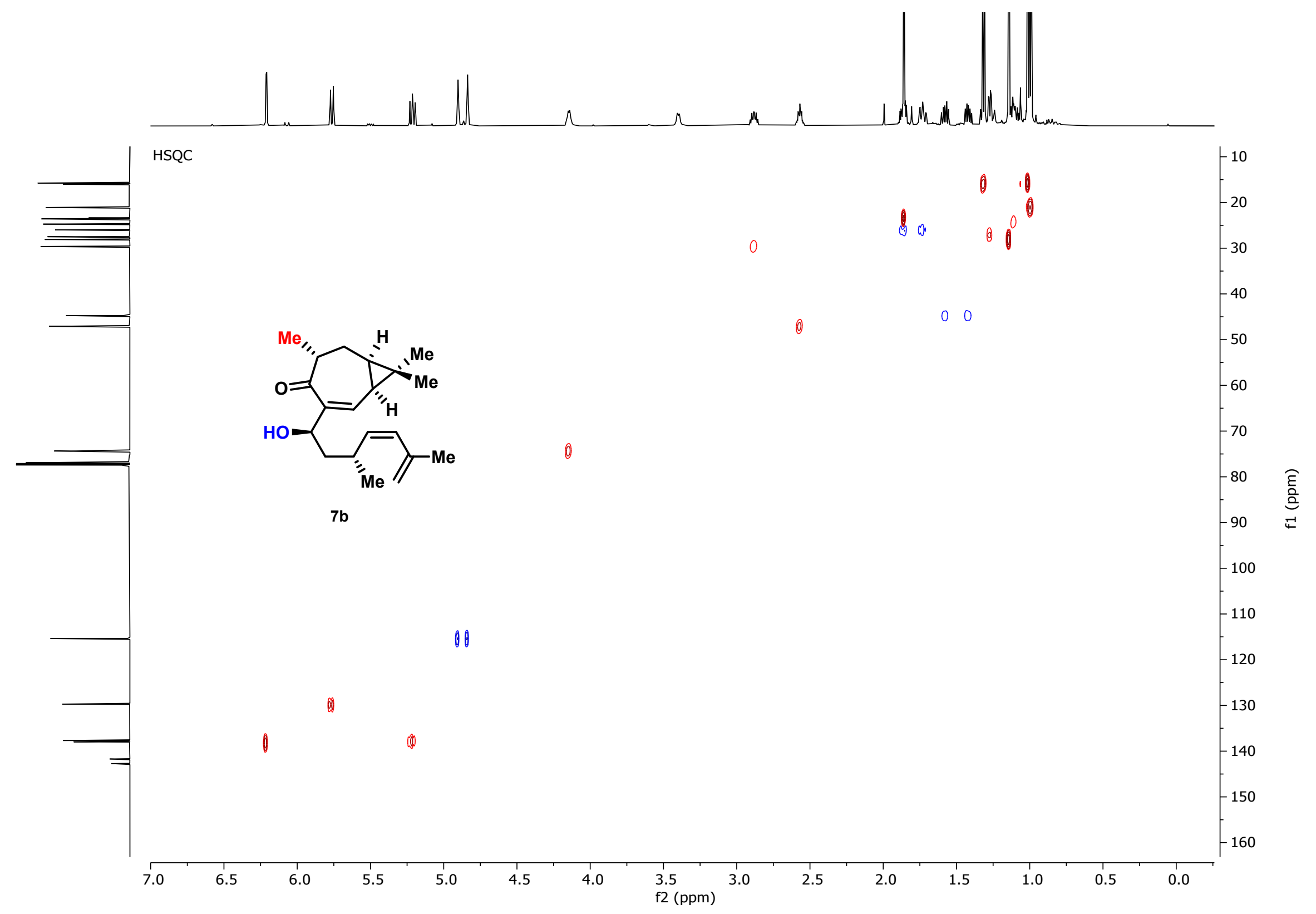




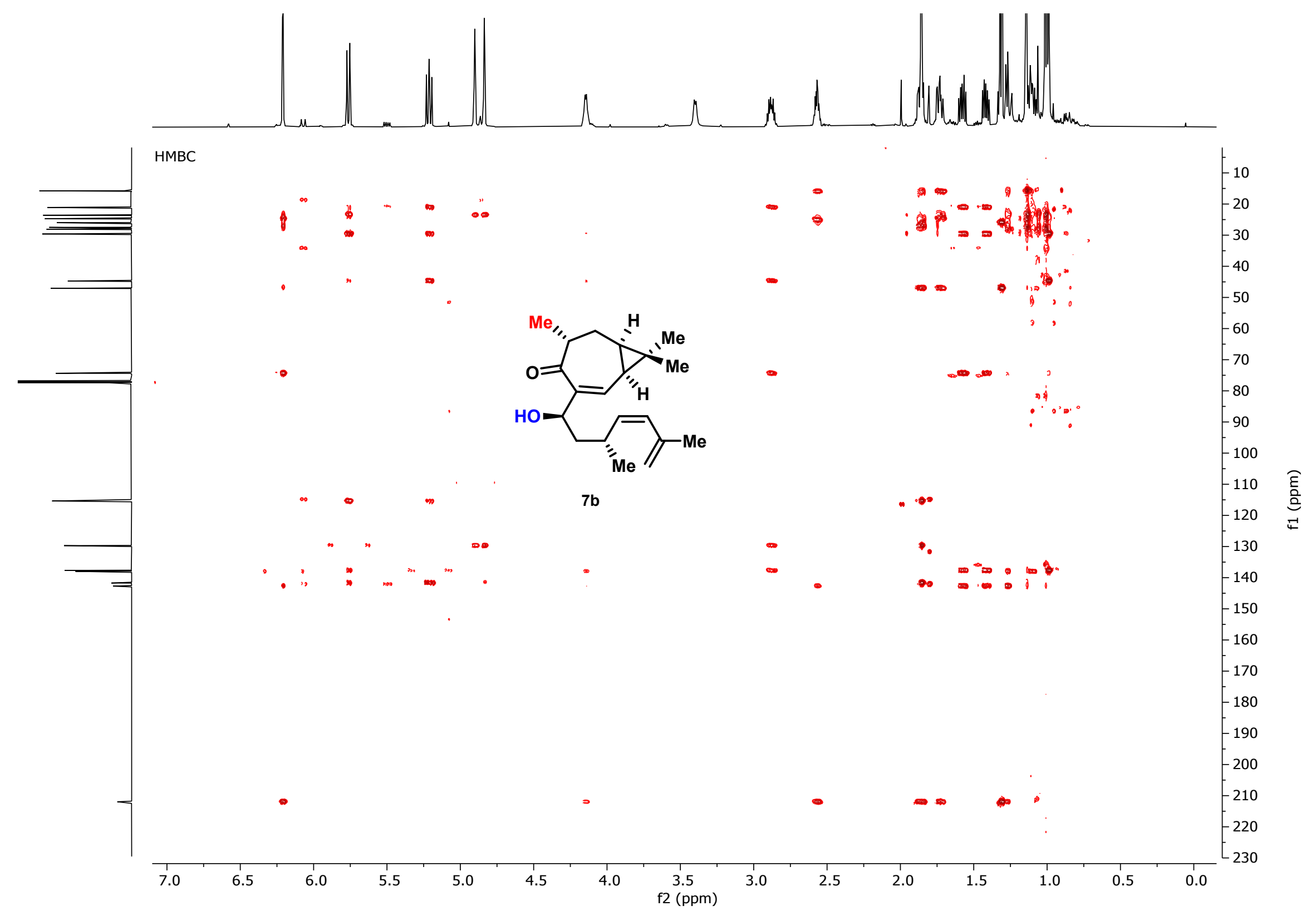




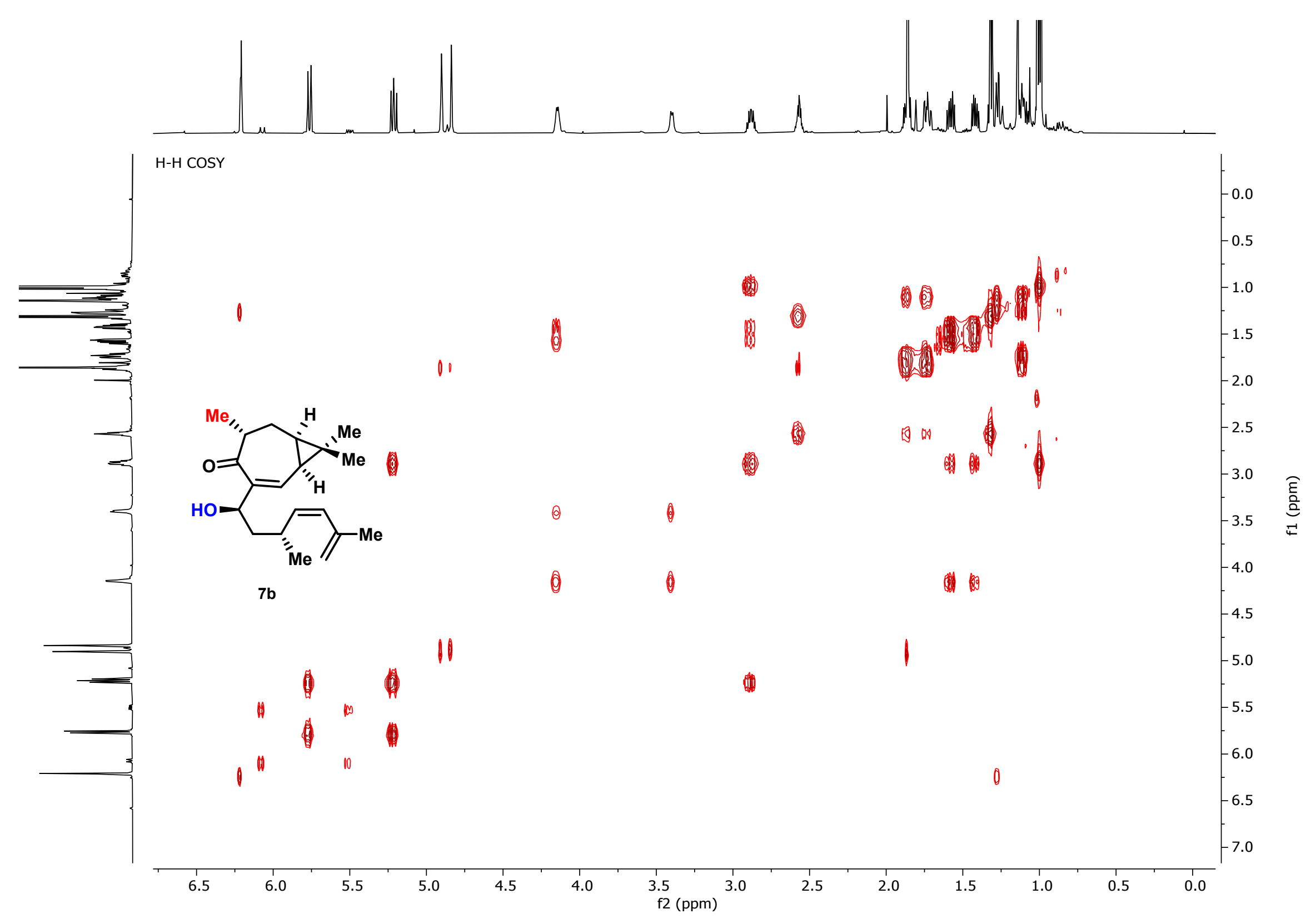



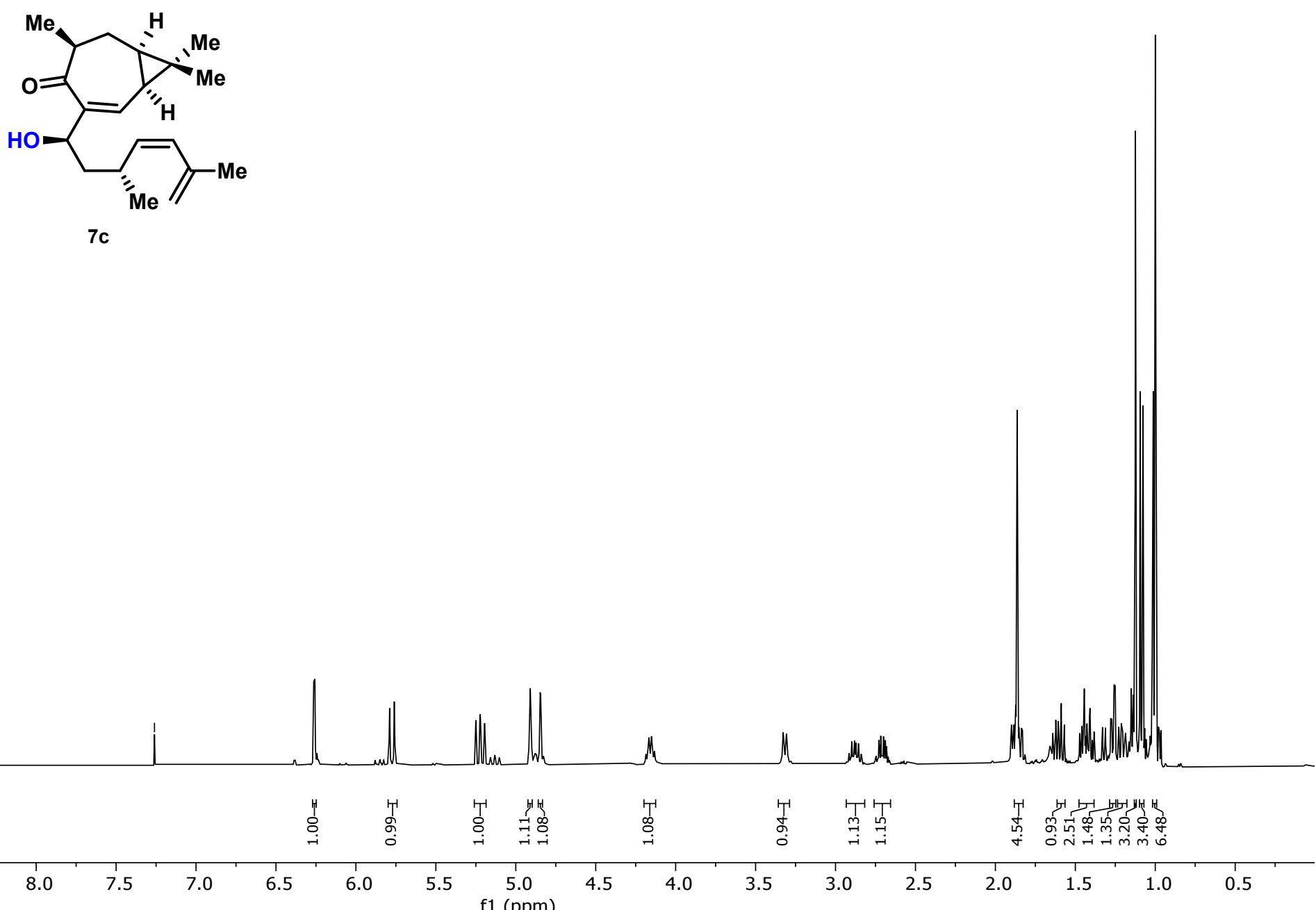

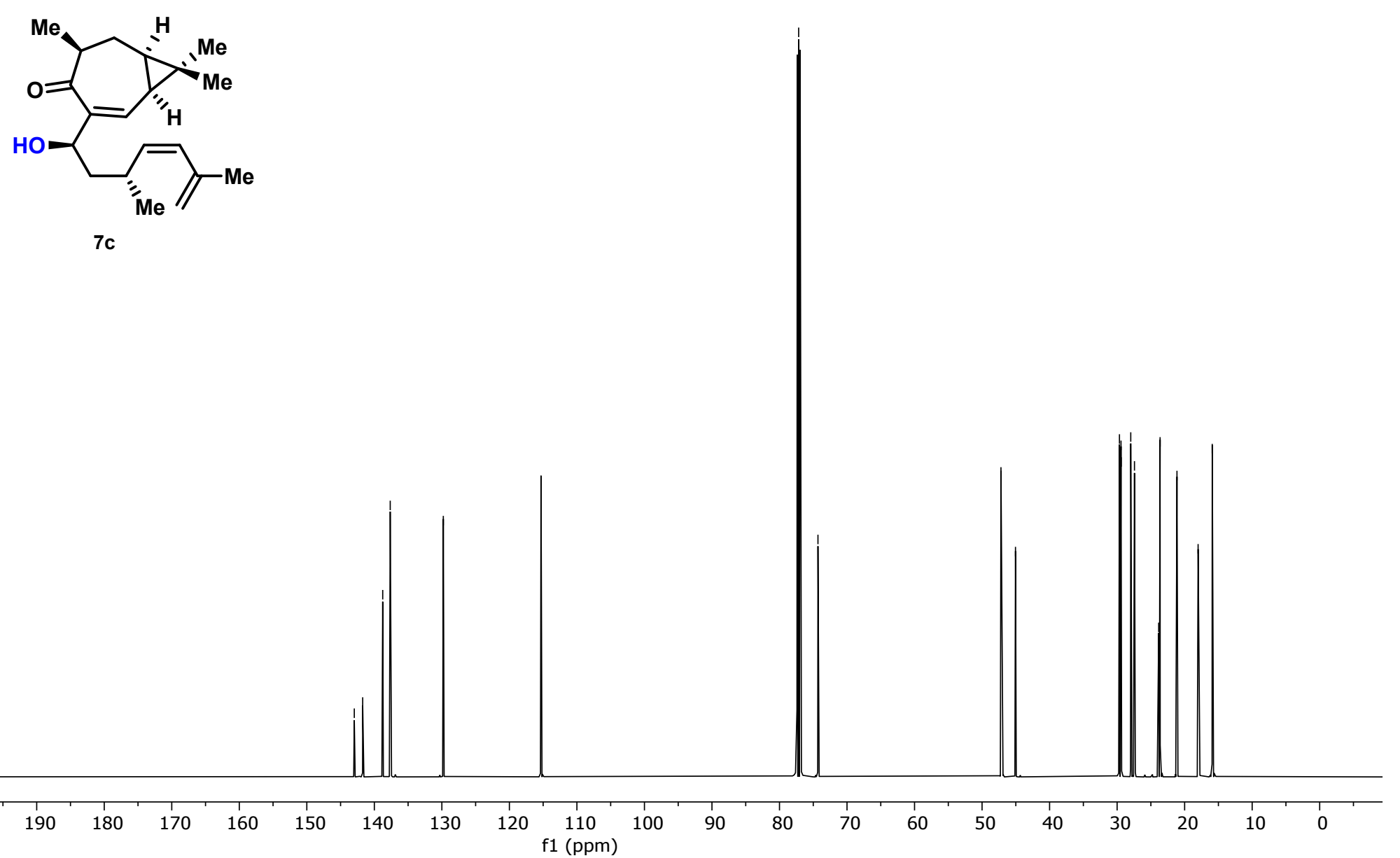


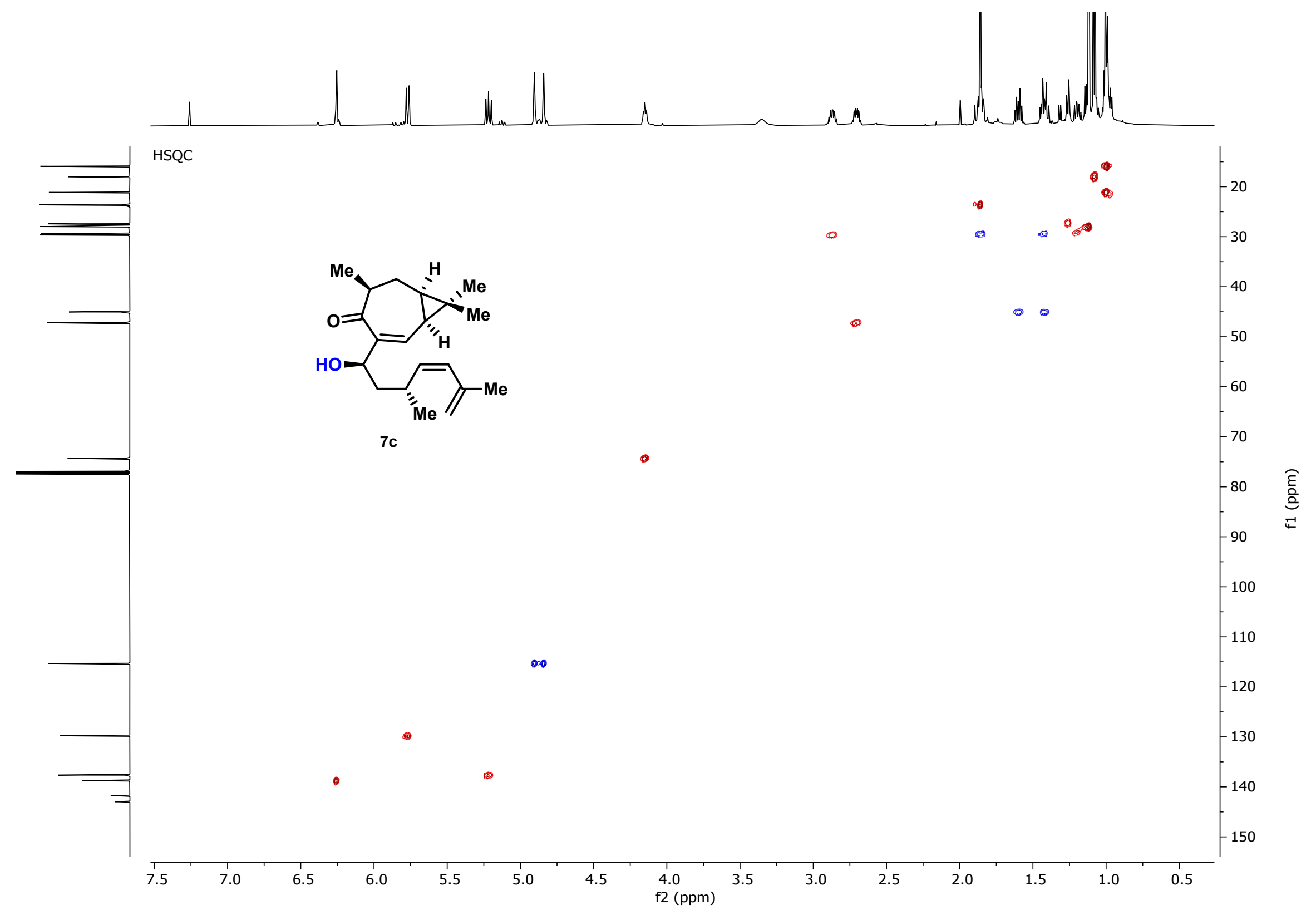




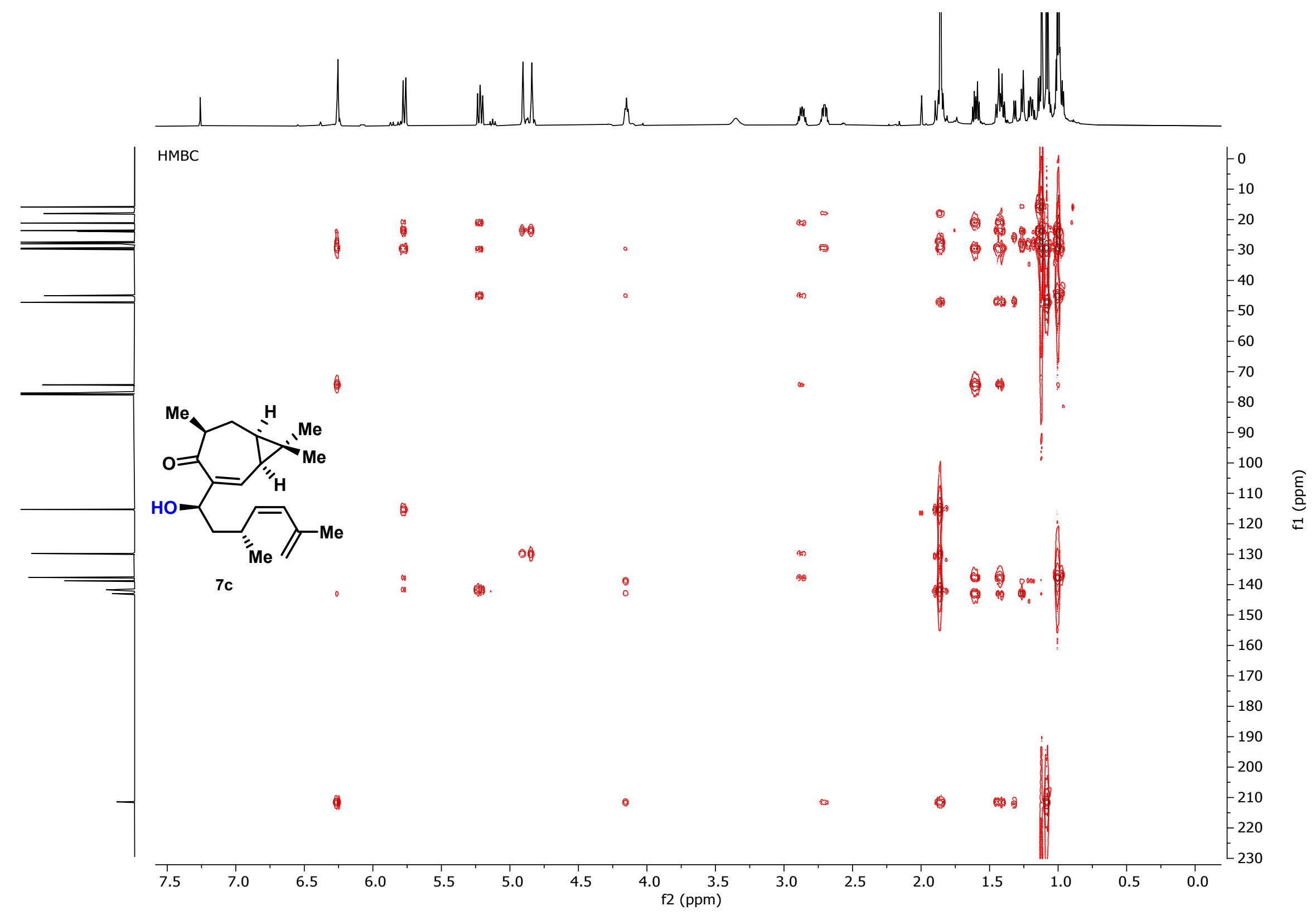




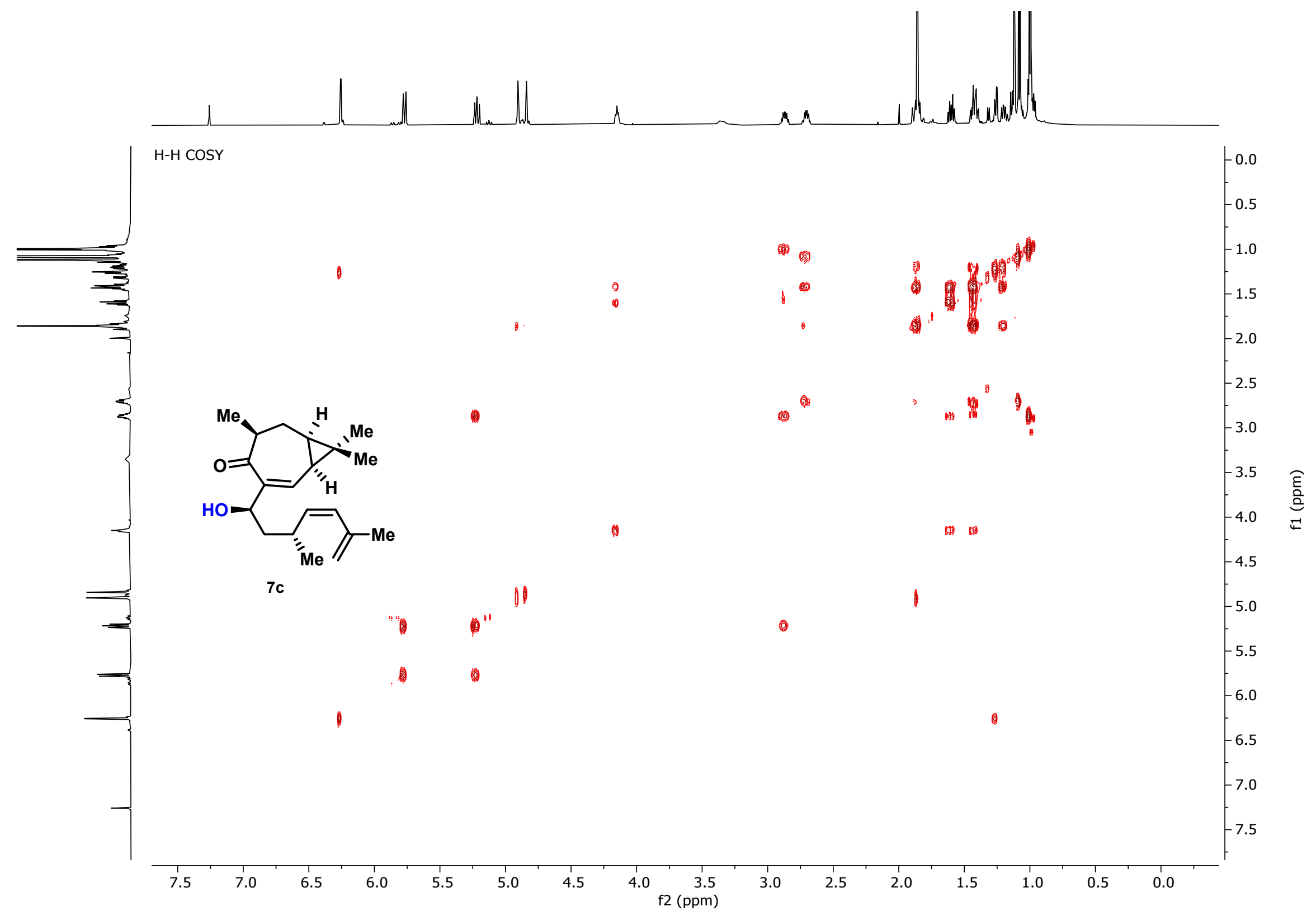



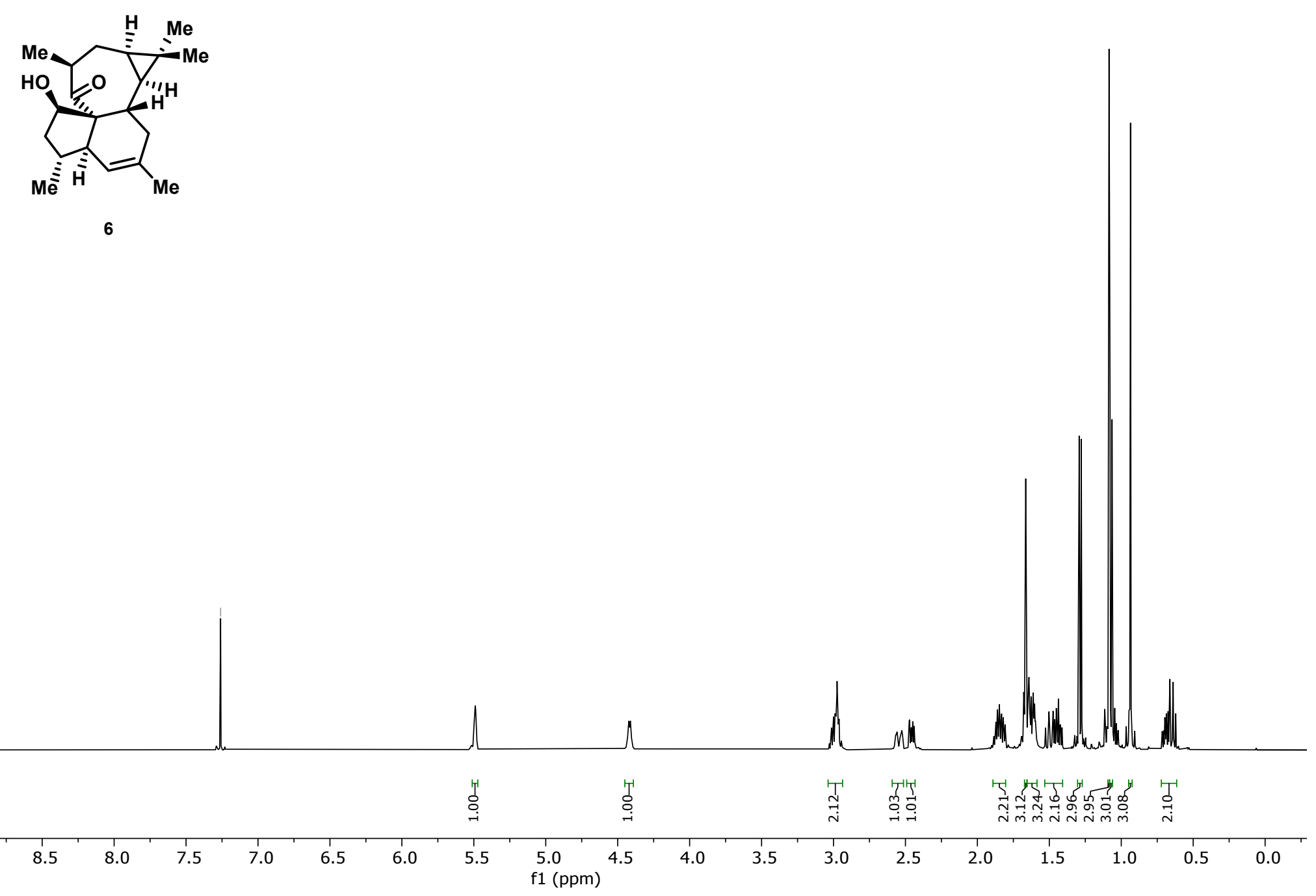


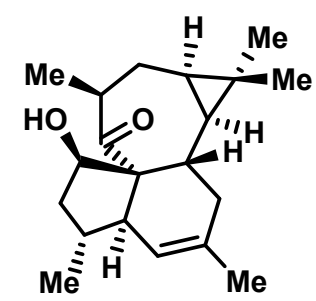

6
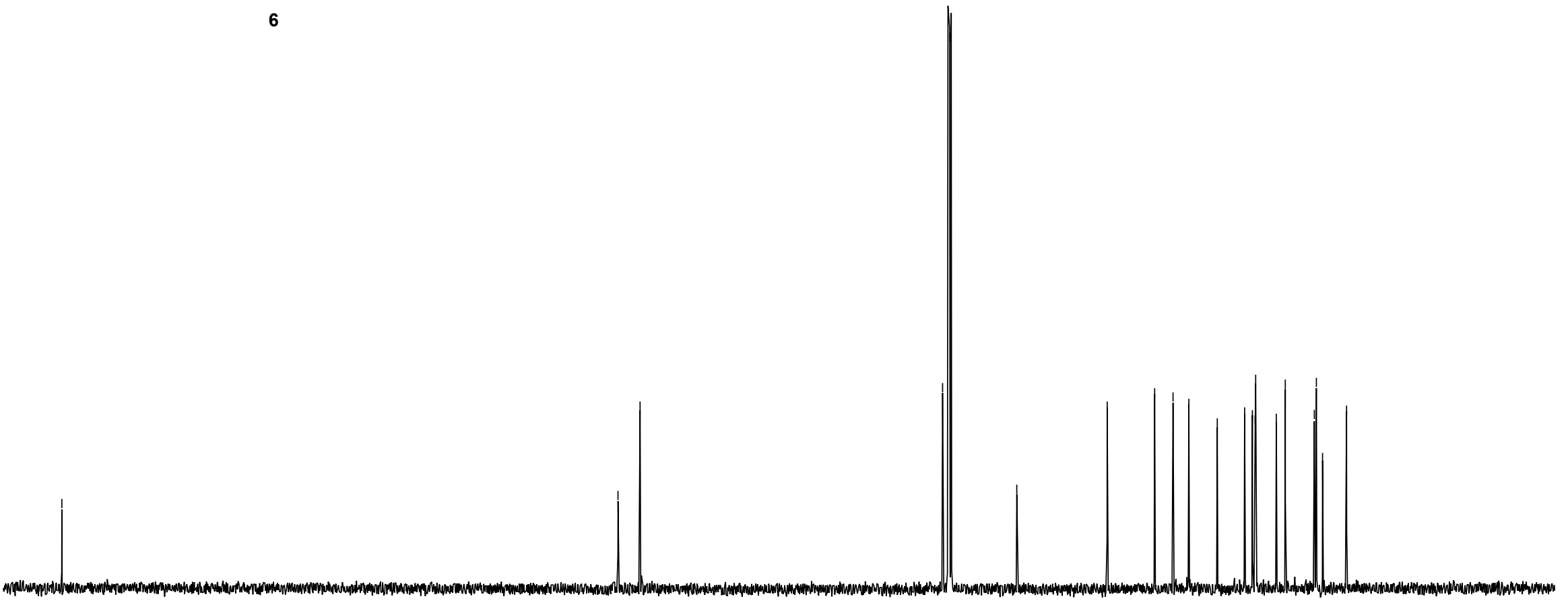

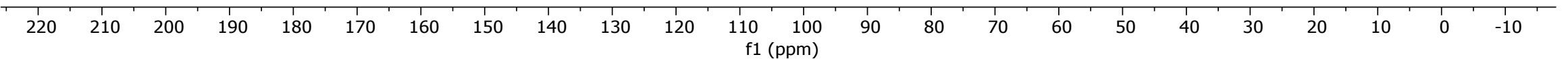




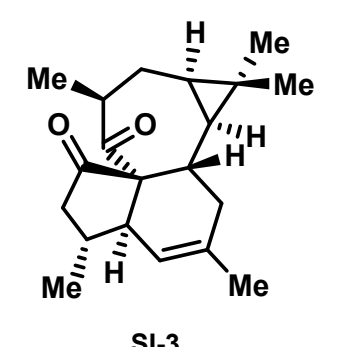

SI-3

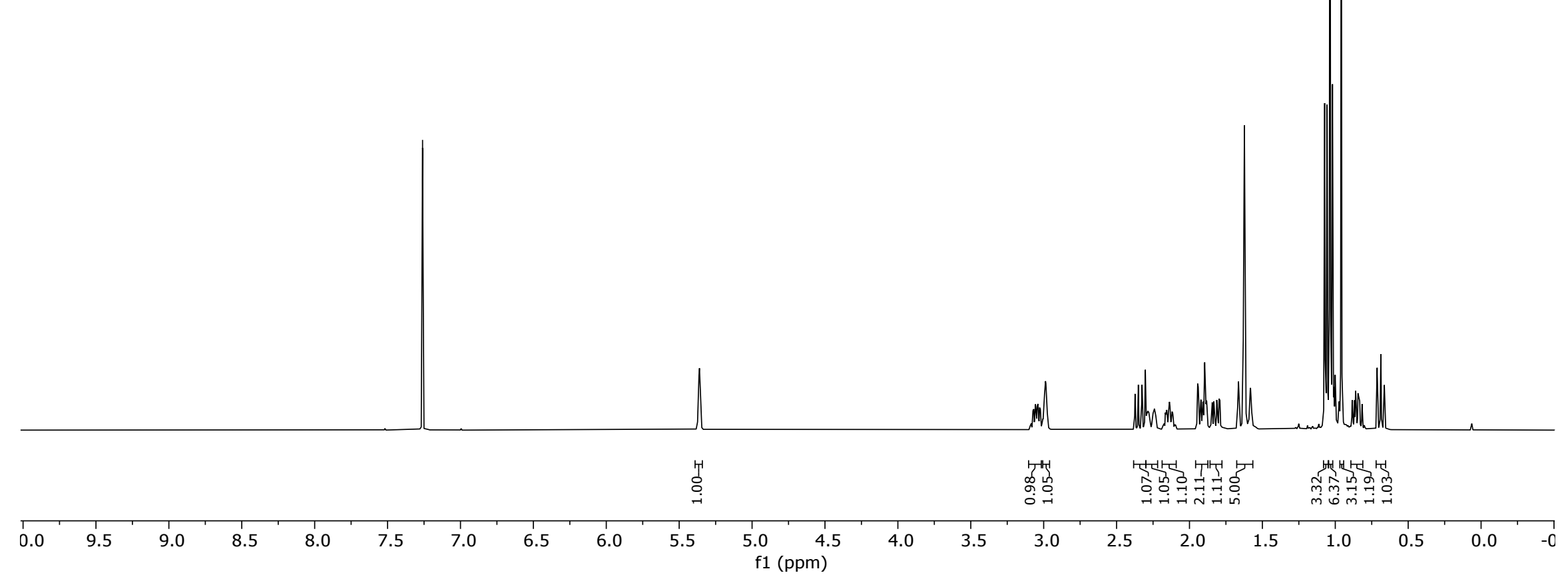




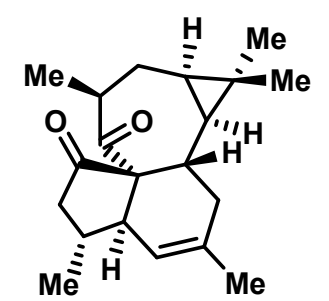

SI-3

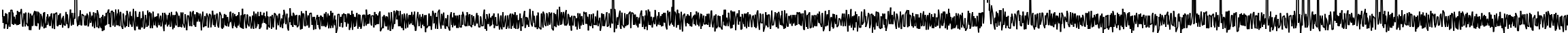

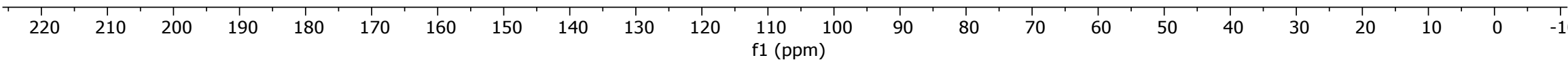



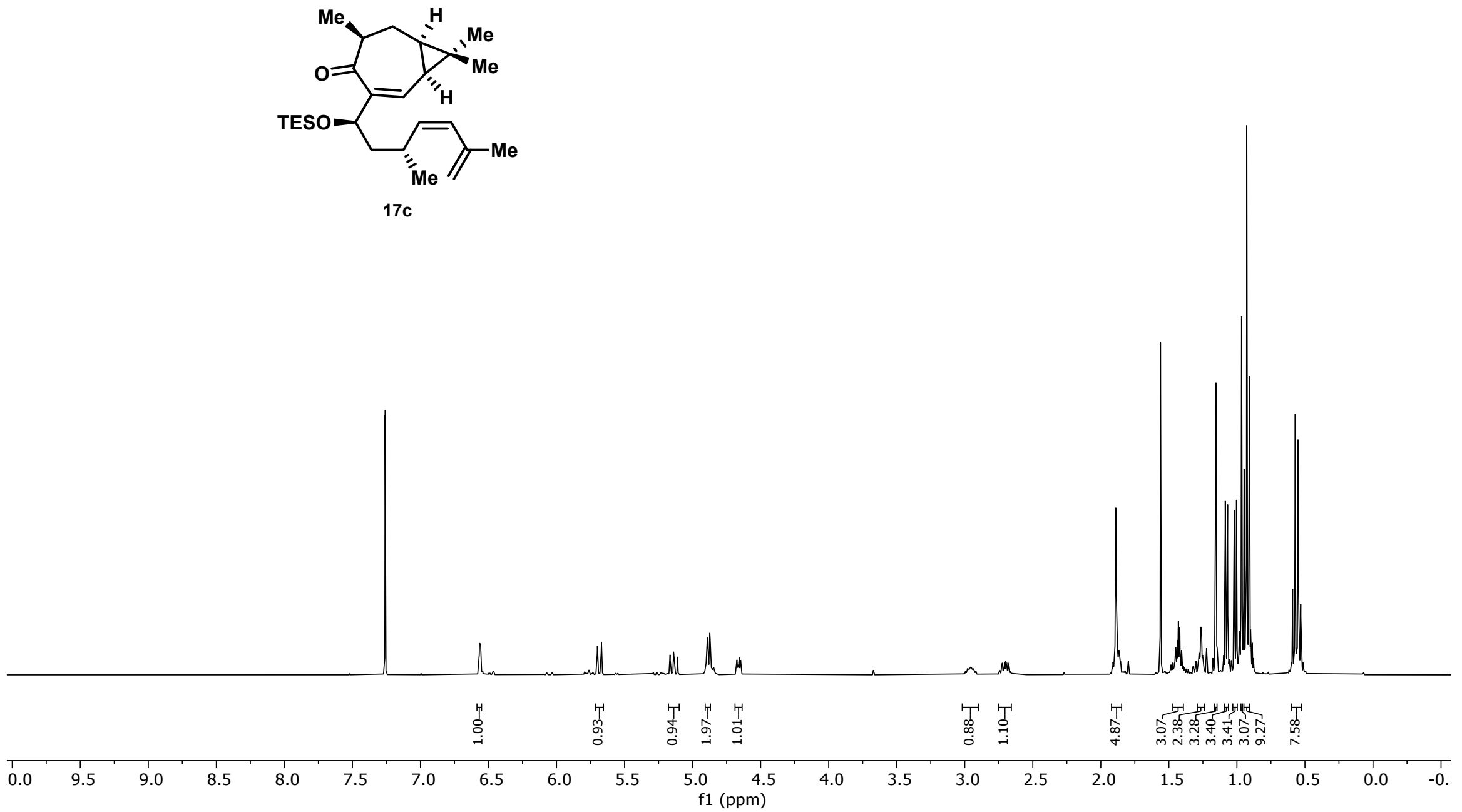

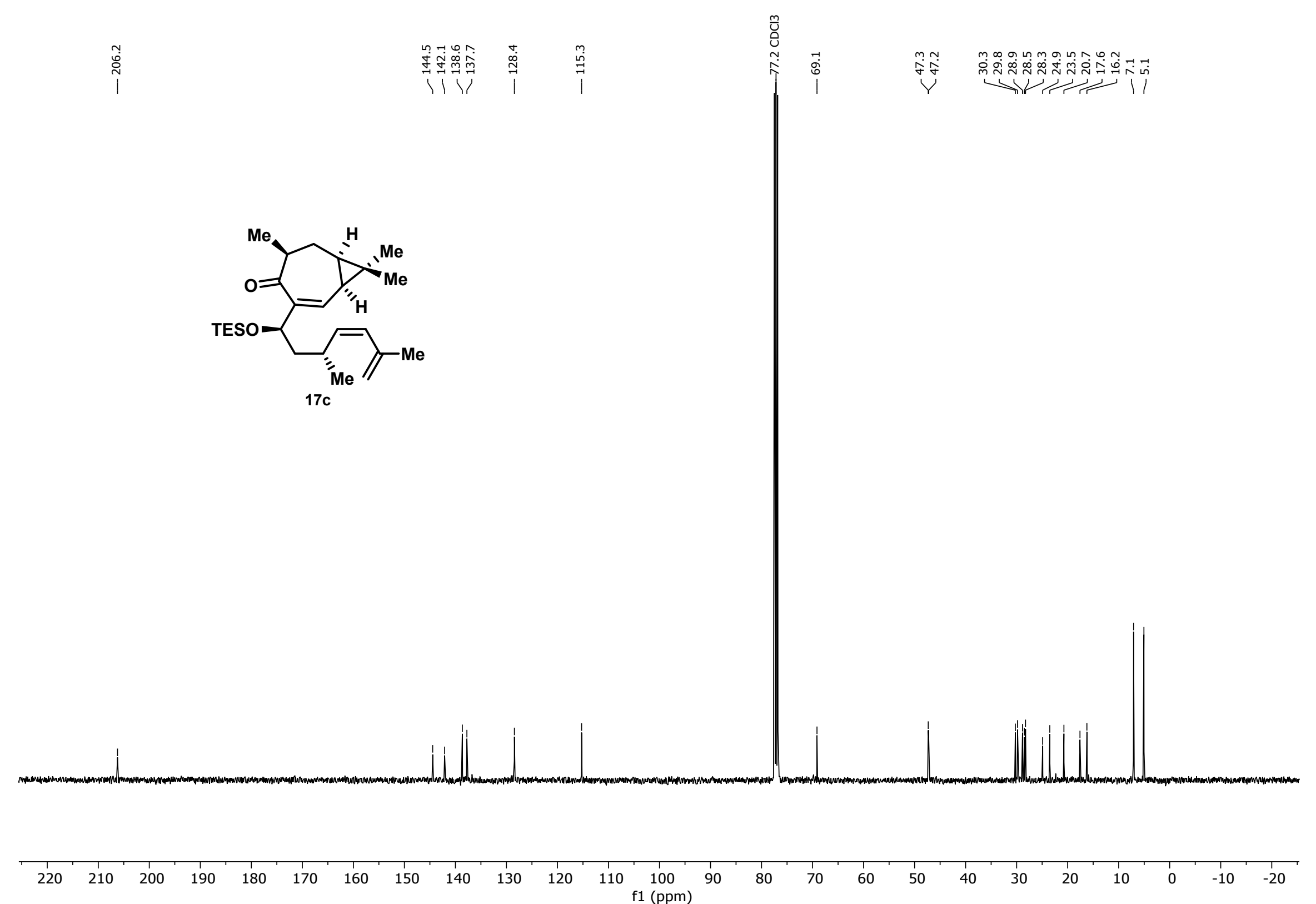


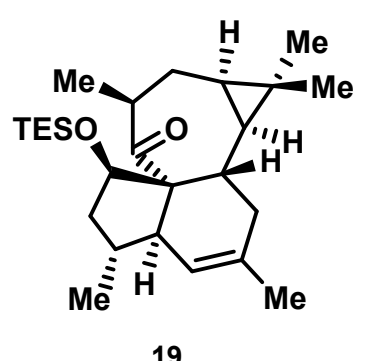

19

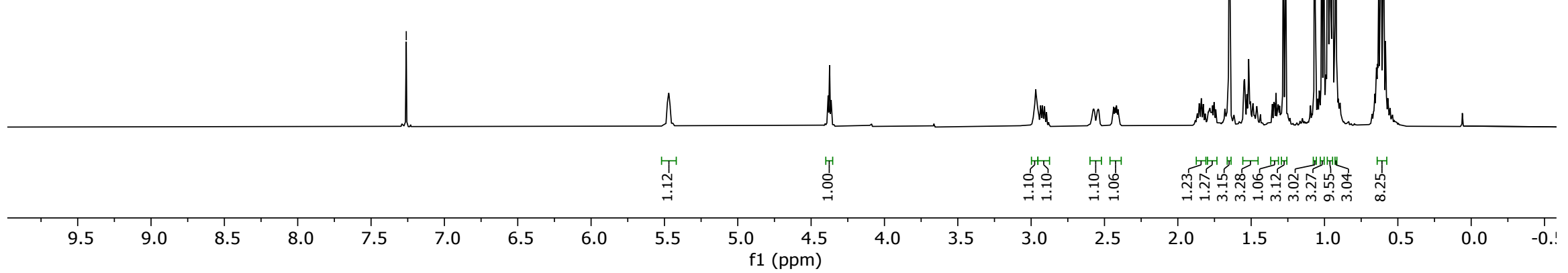




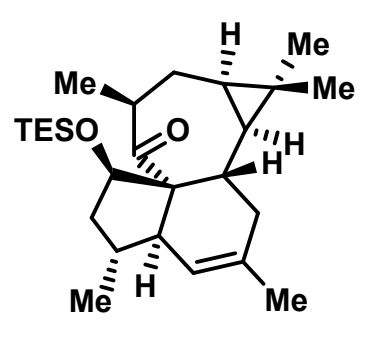

19

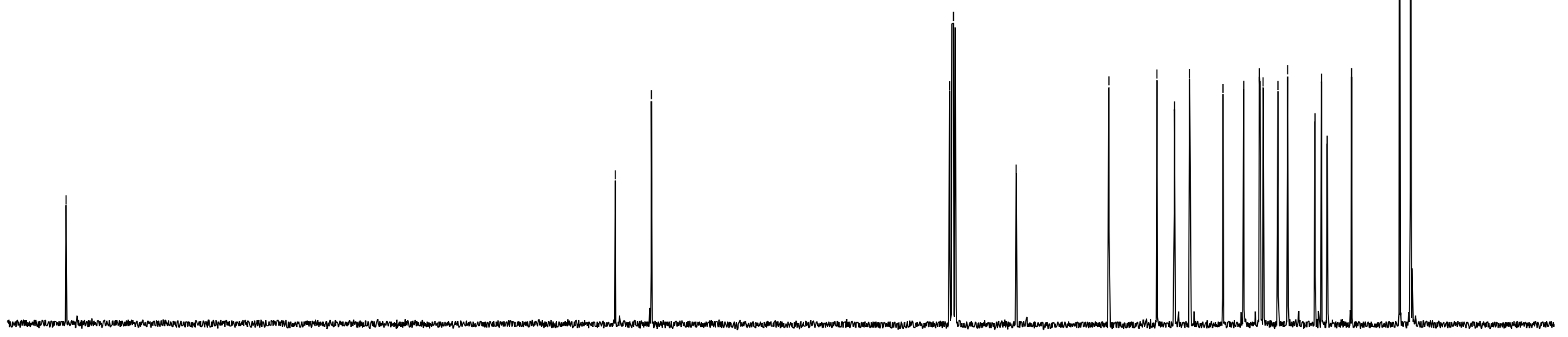

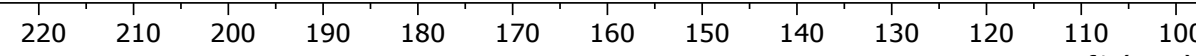

f1 (ppm)
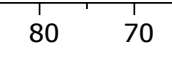

60

$50 \quad 40$

30

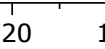

$10 \quad 0 \quad-10$ 


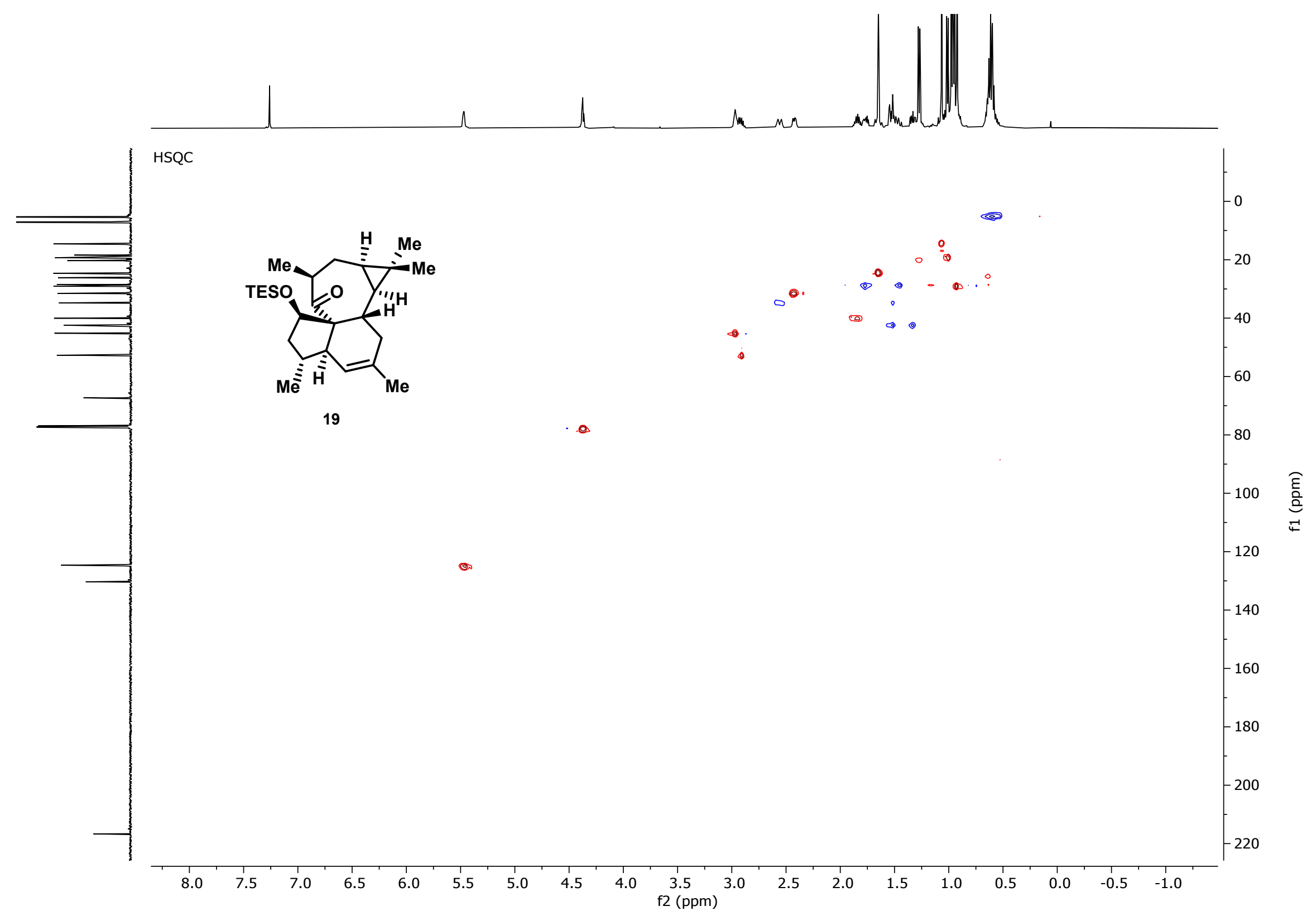




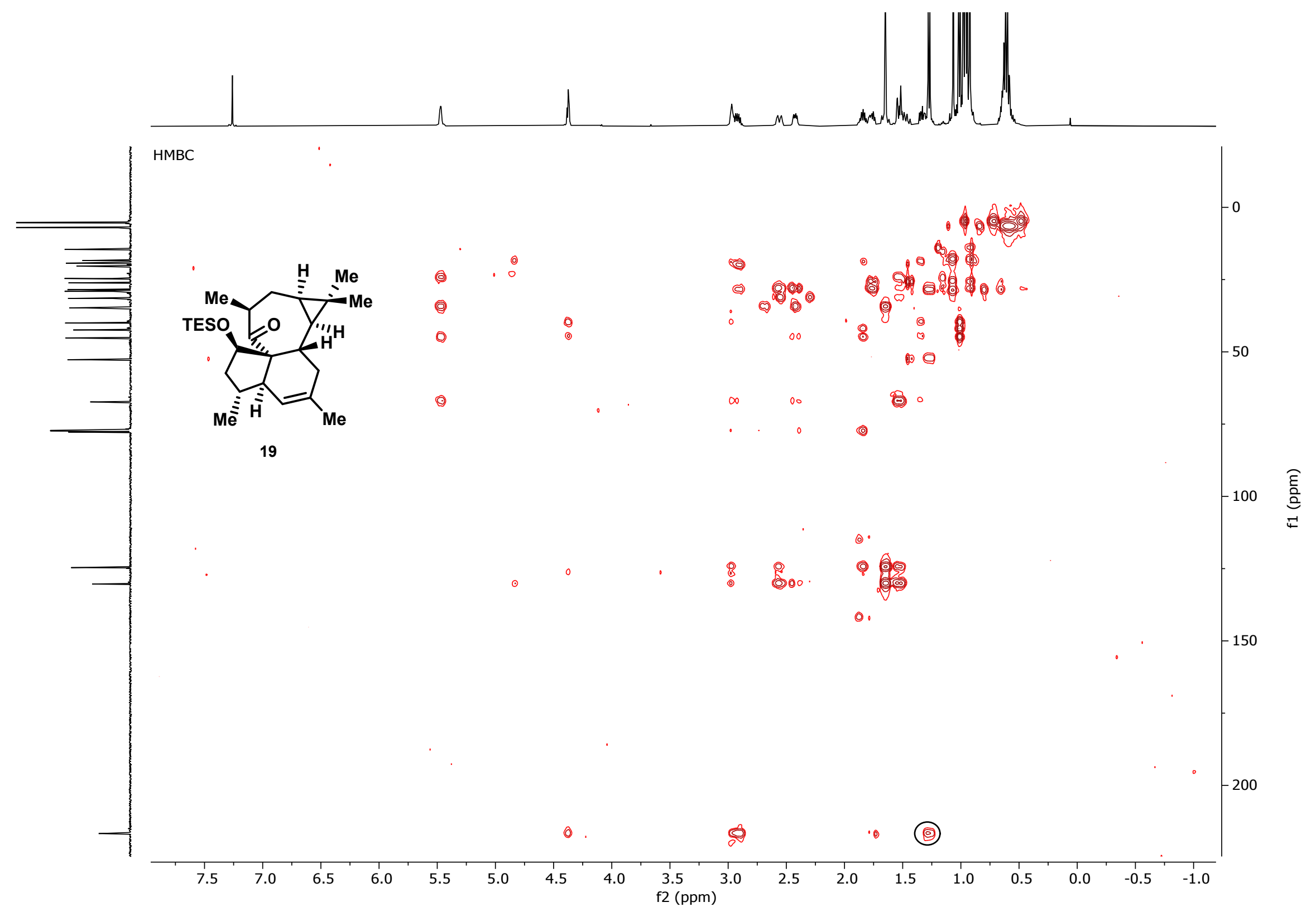




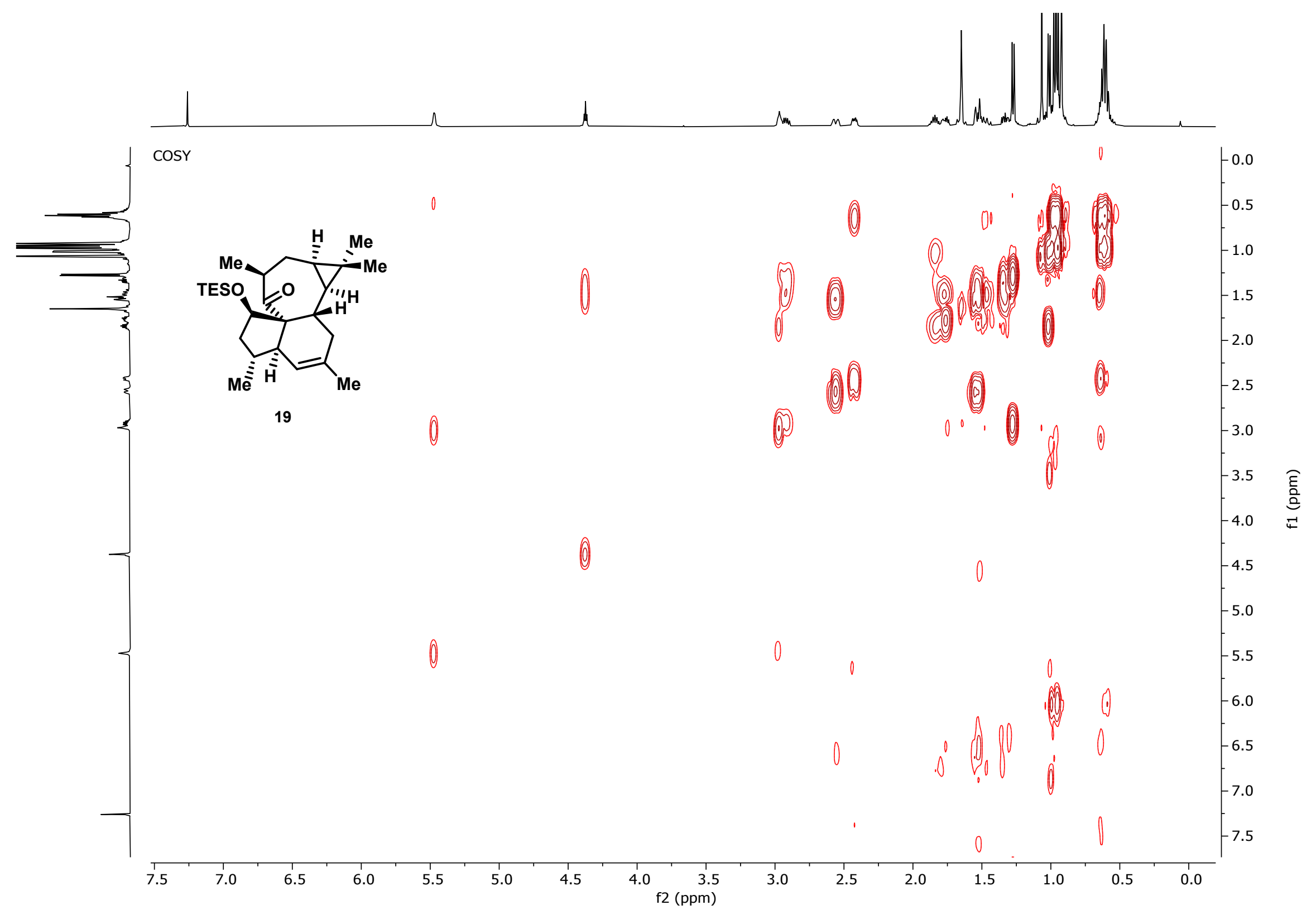




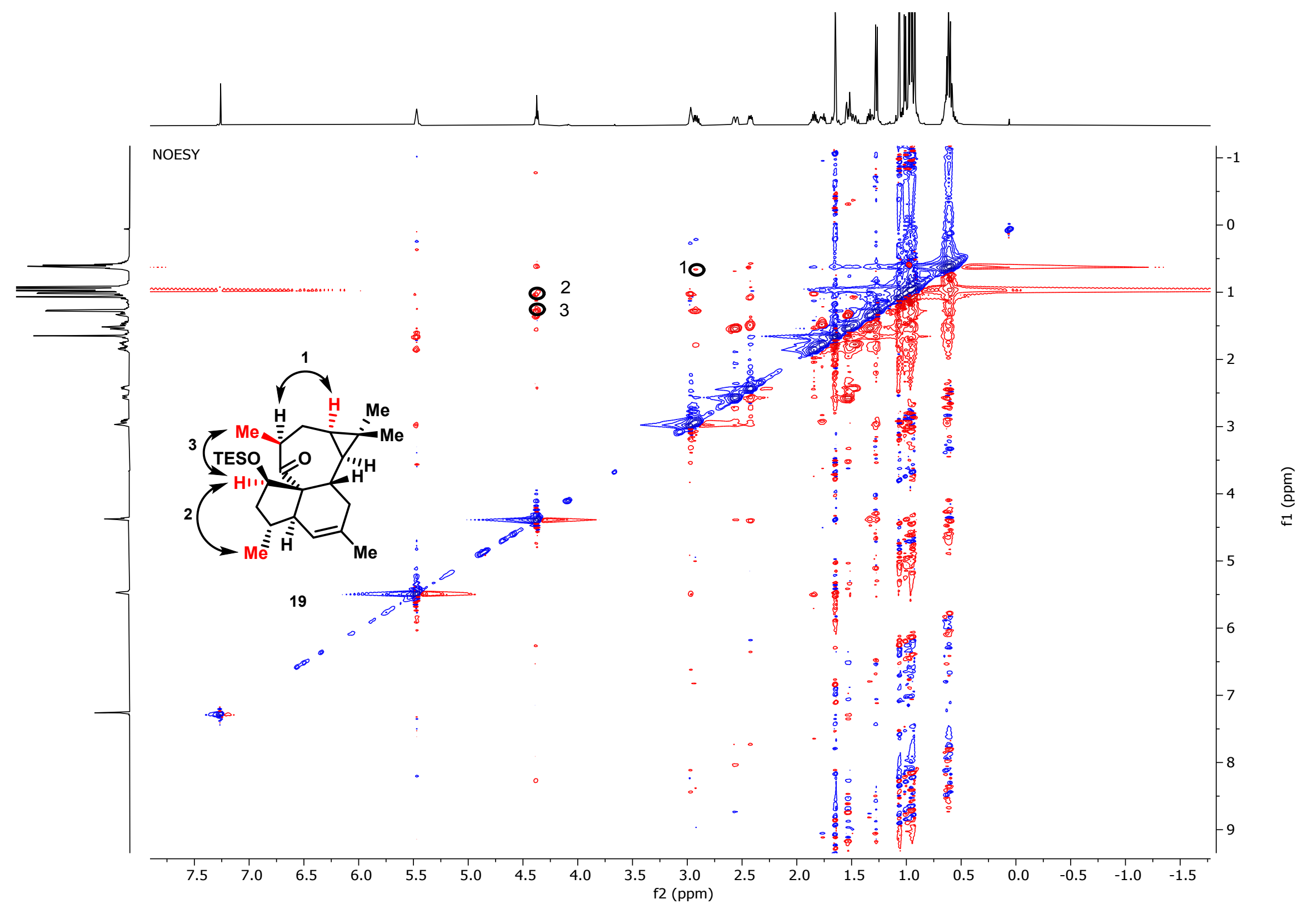



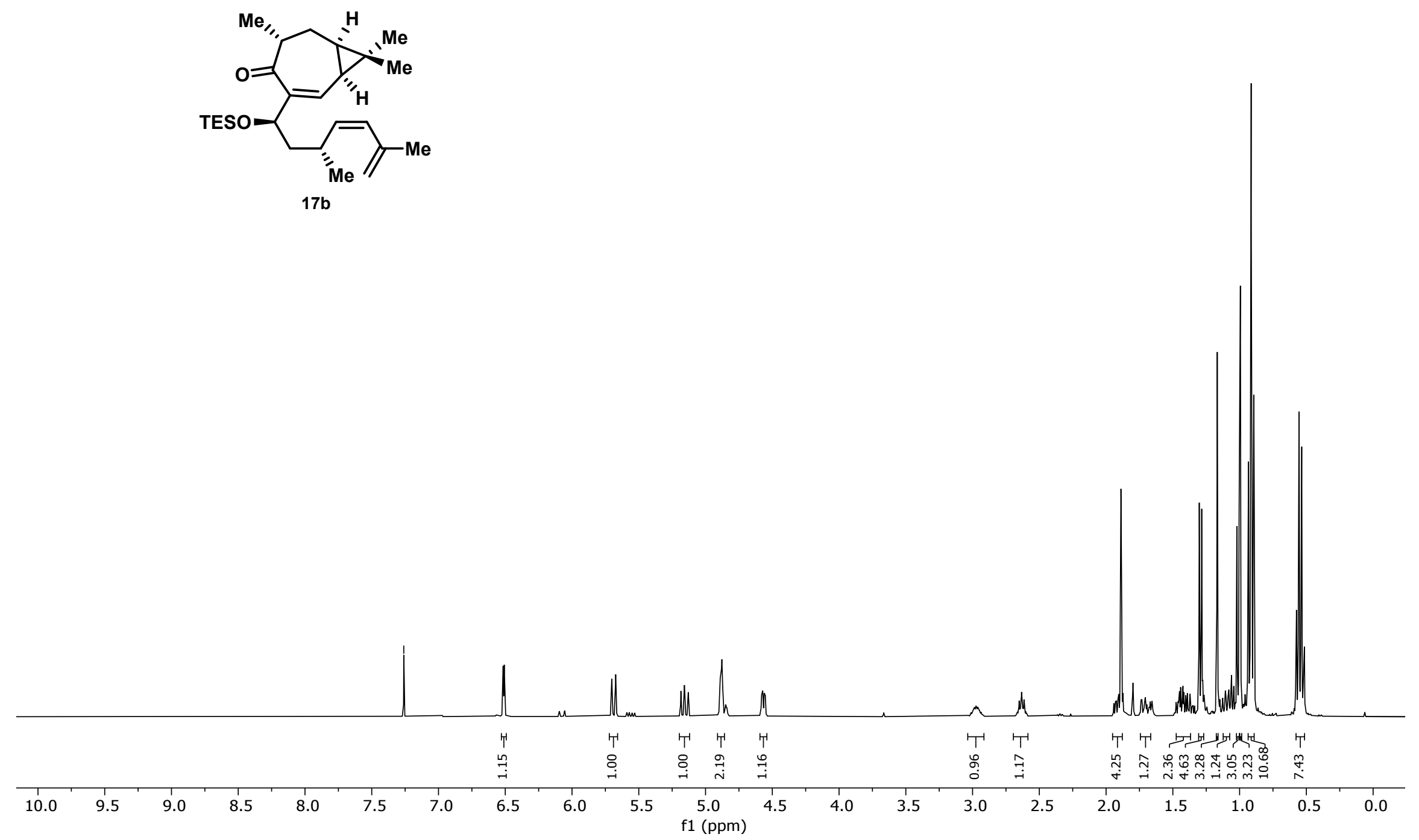


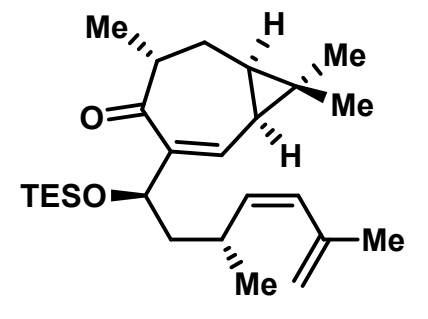

17b

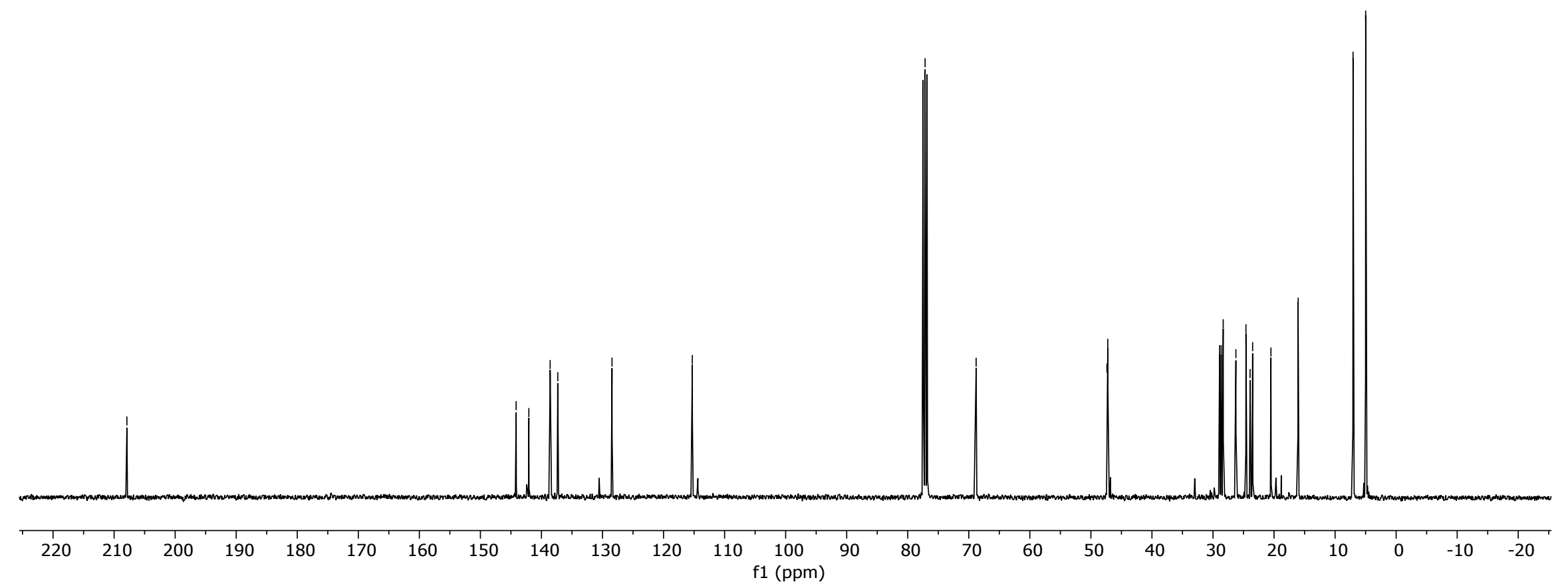



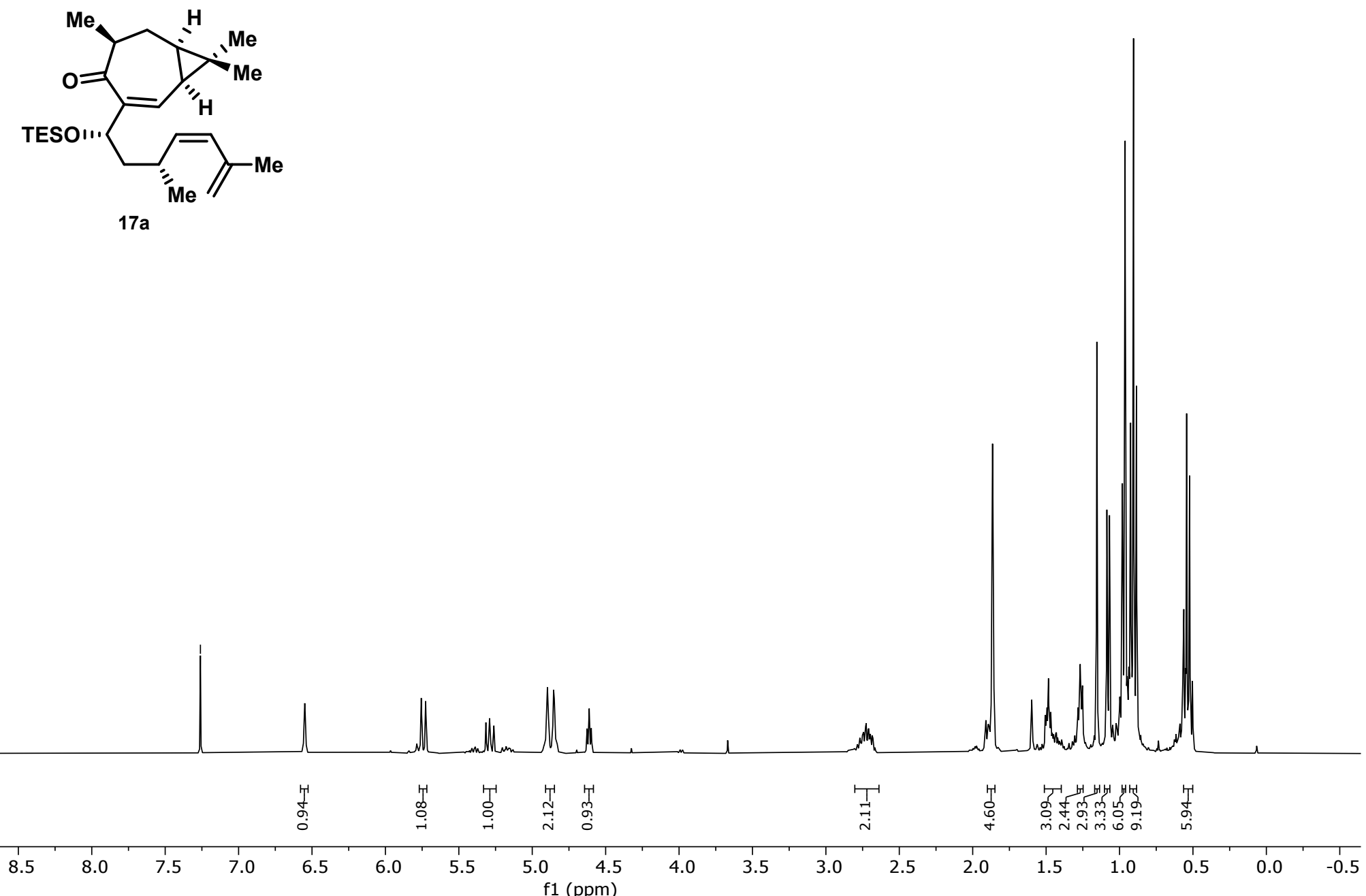


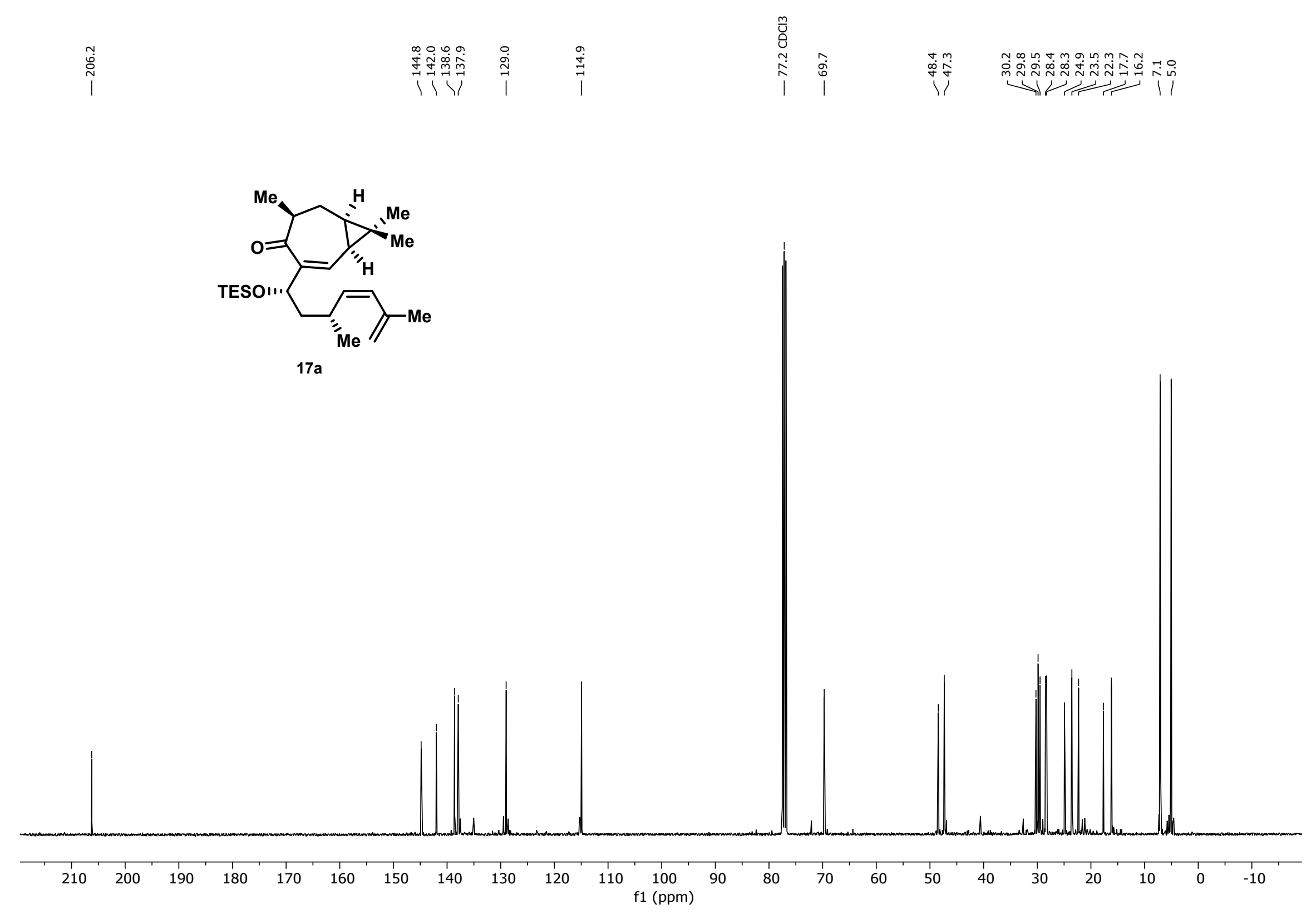




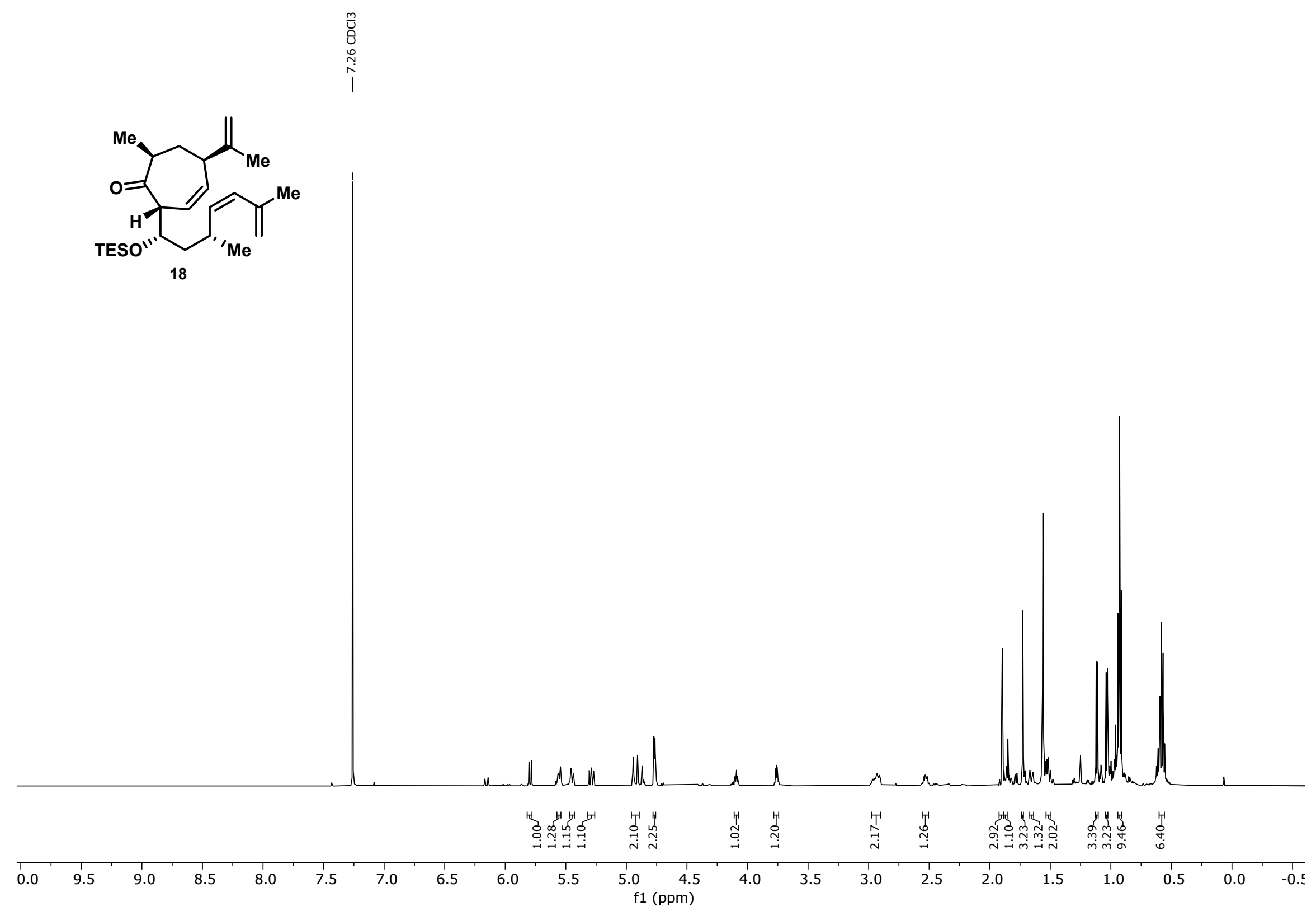




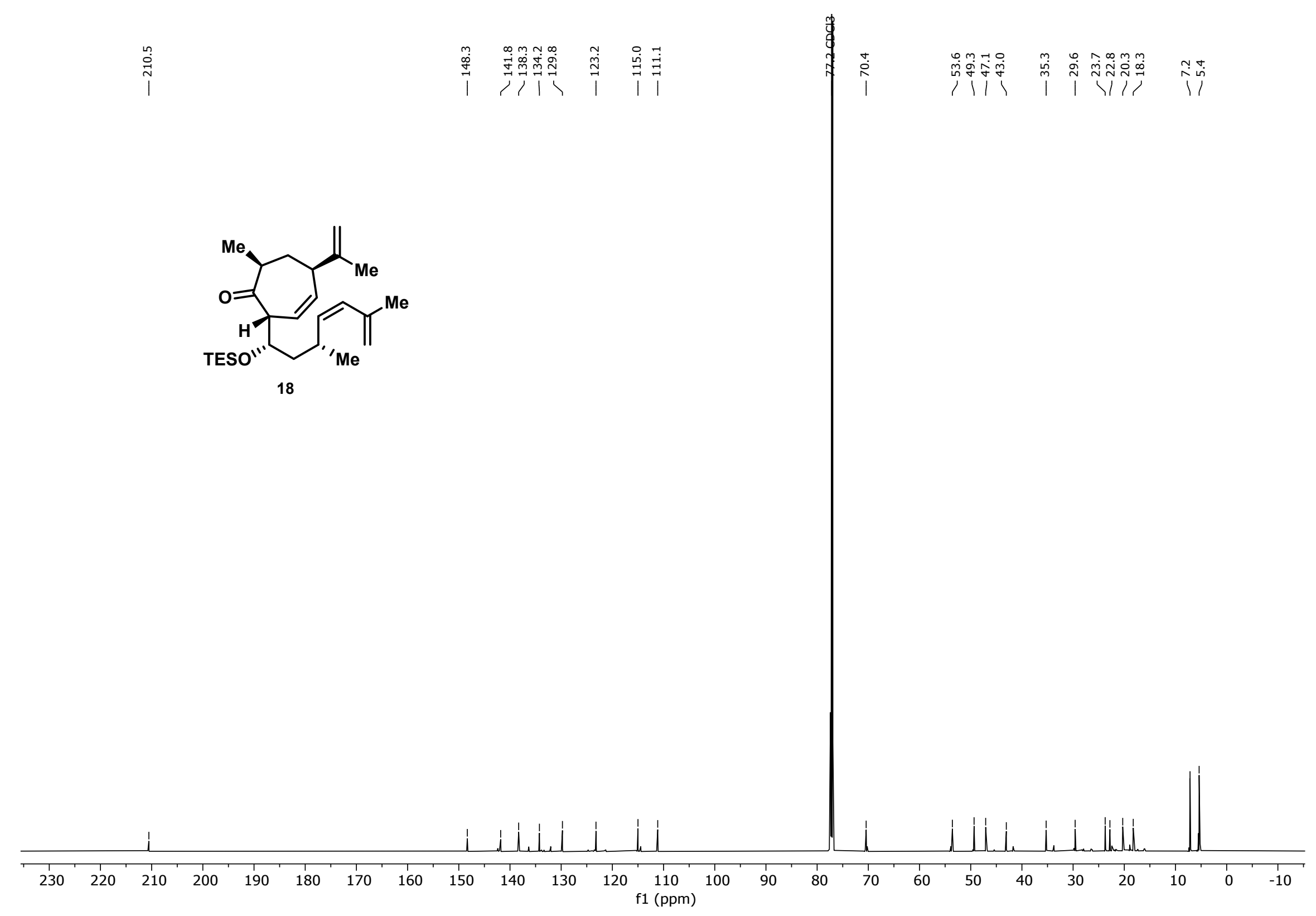




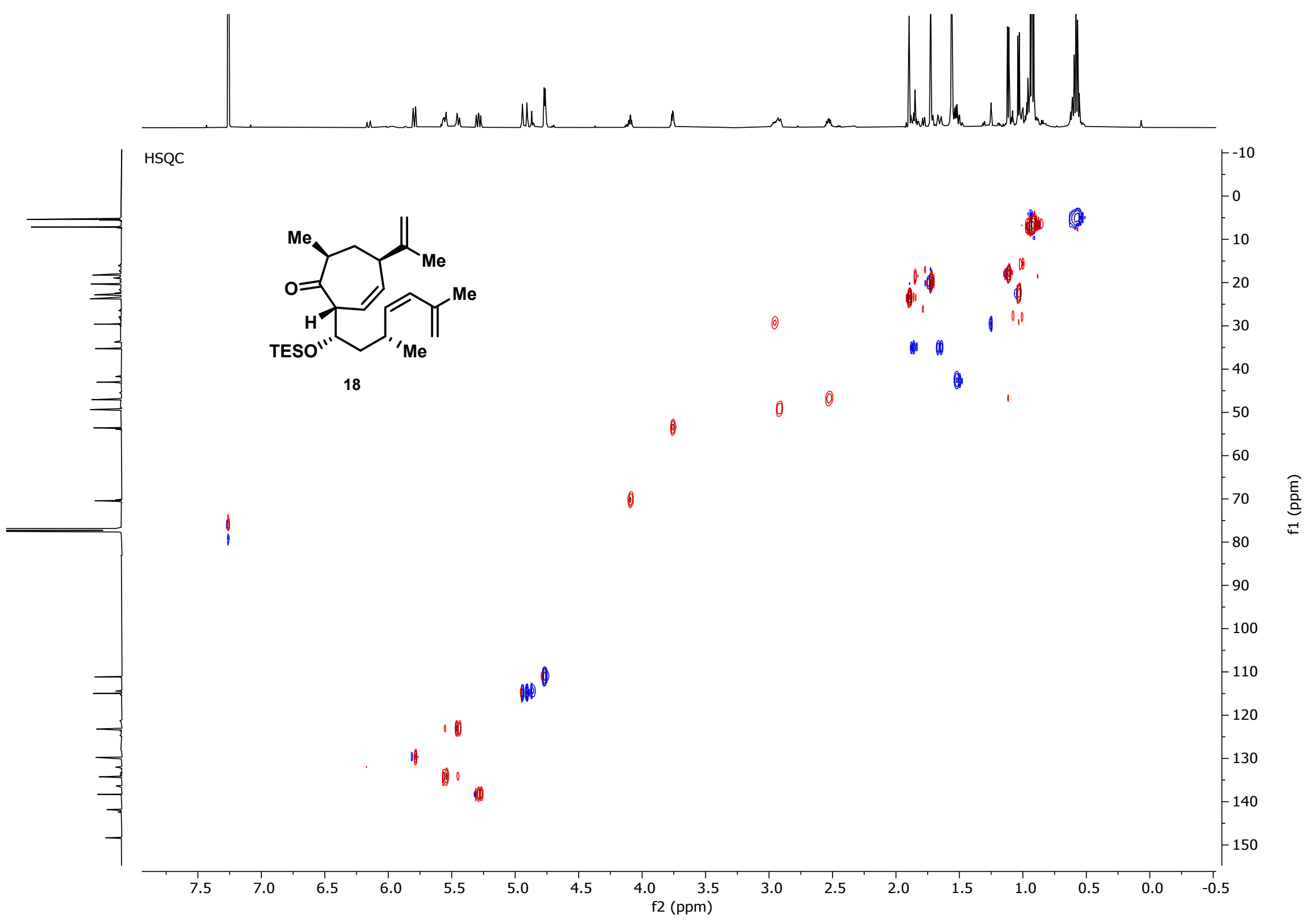




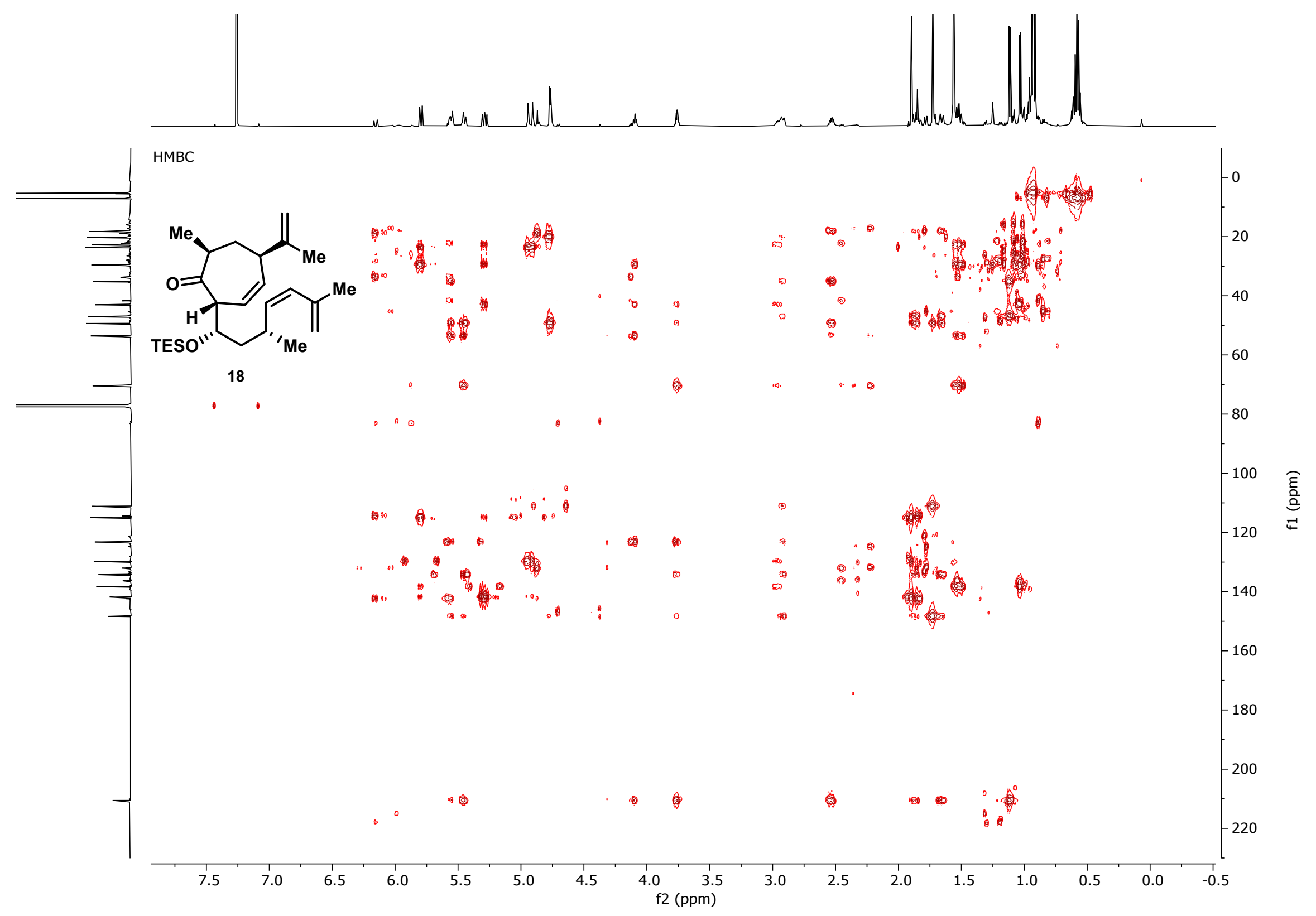




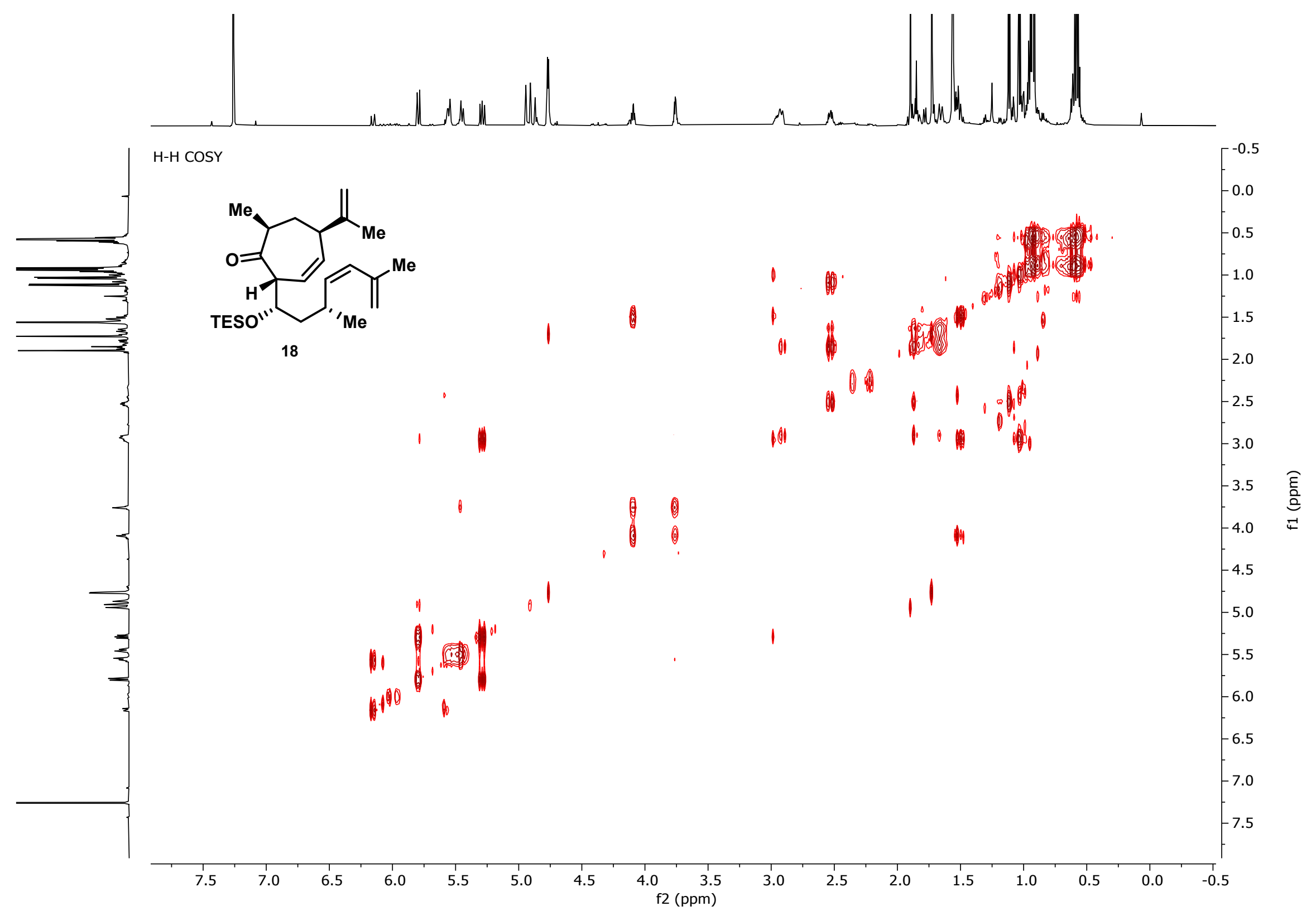




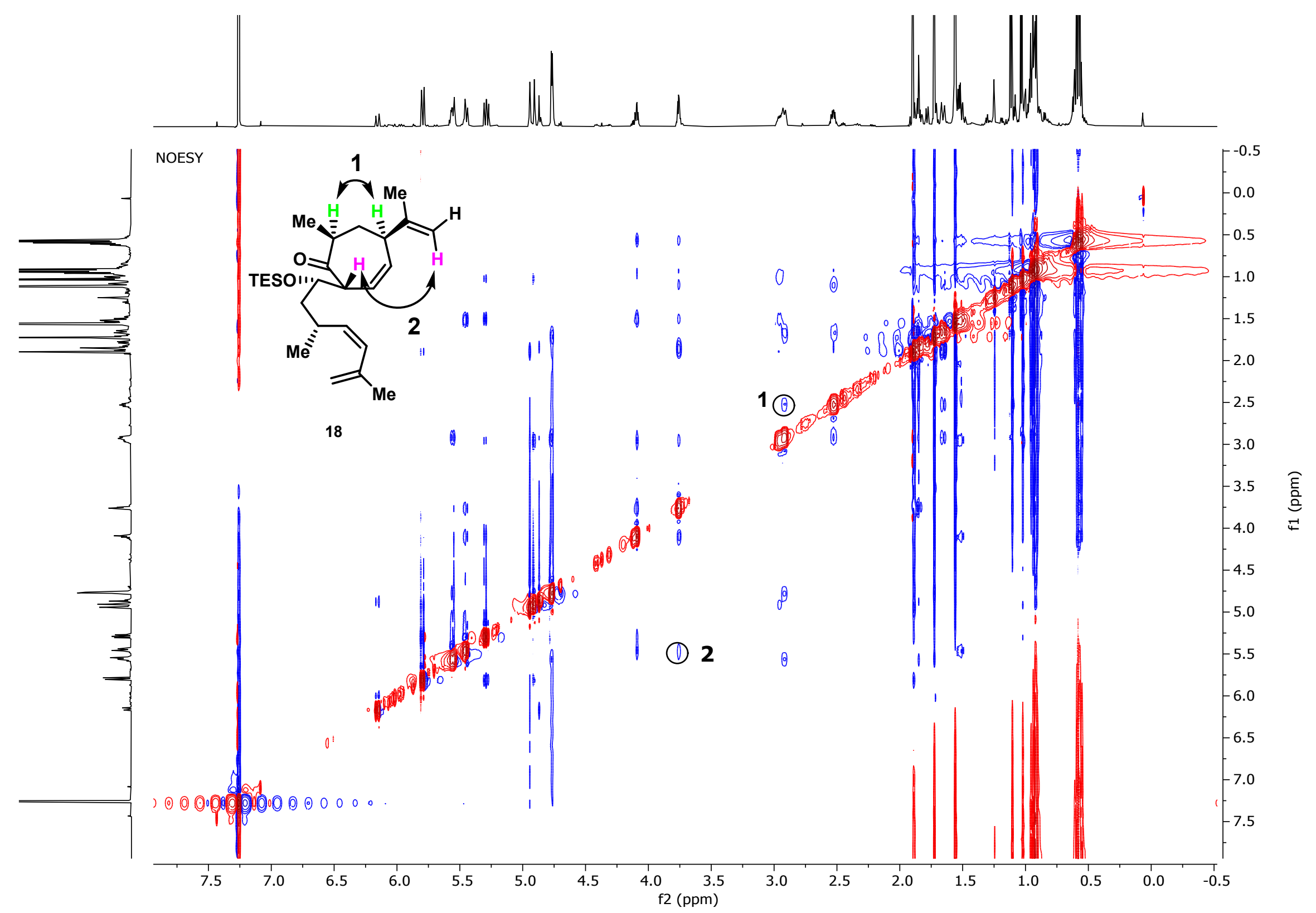




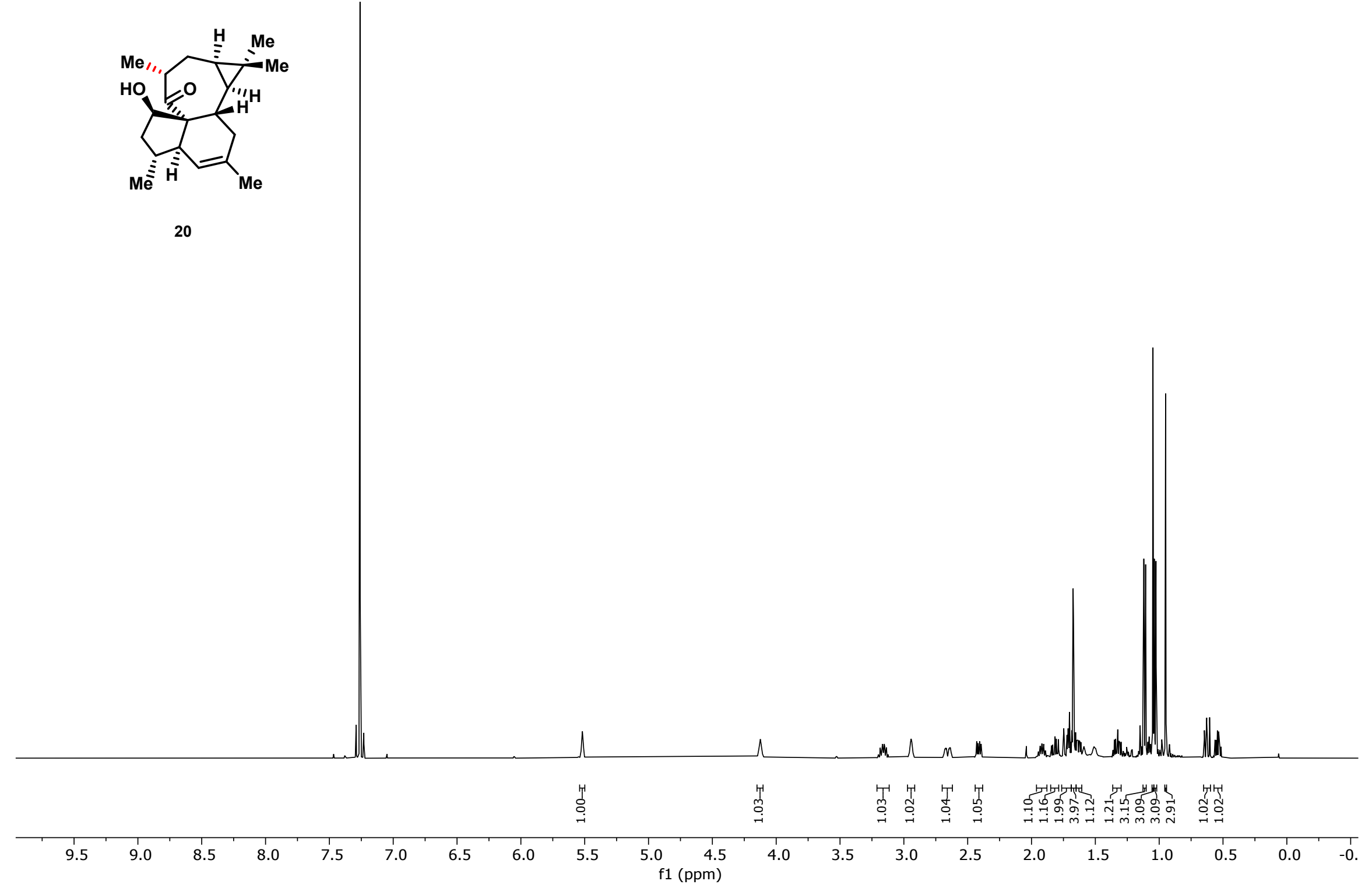




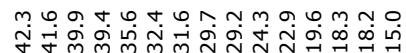

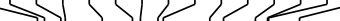

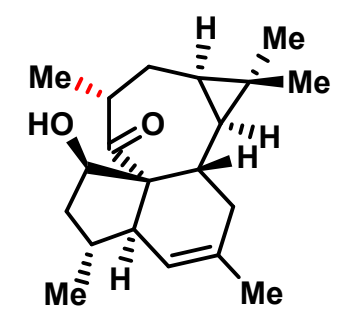

20

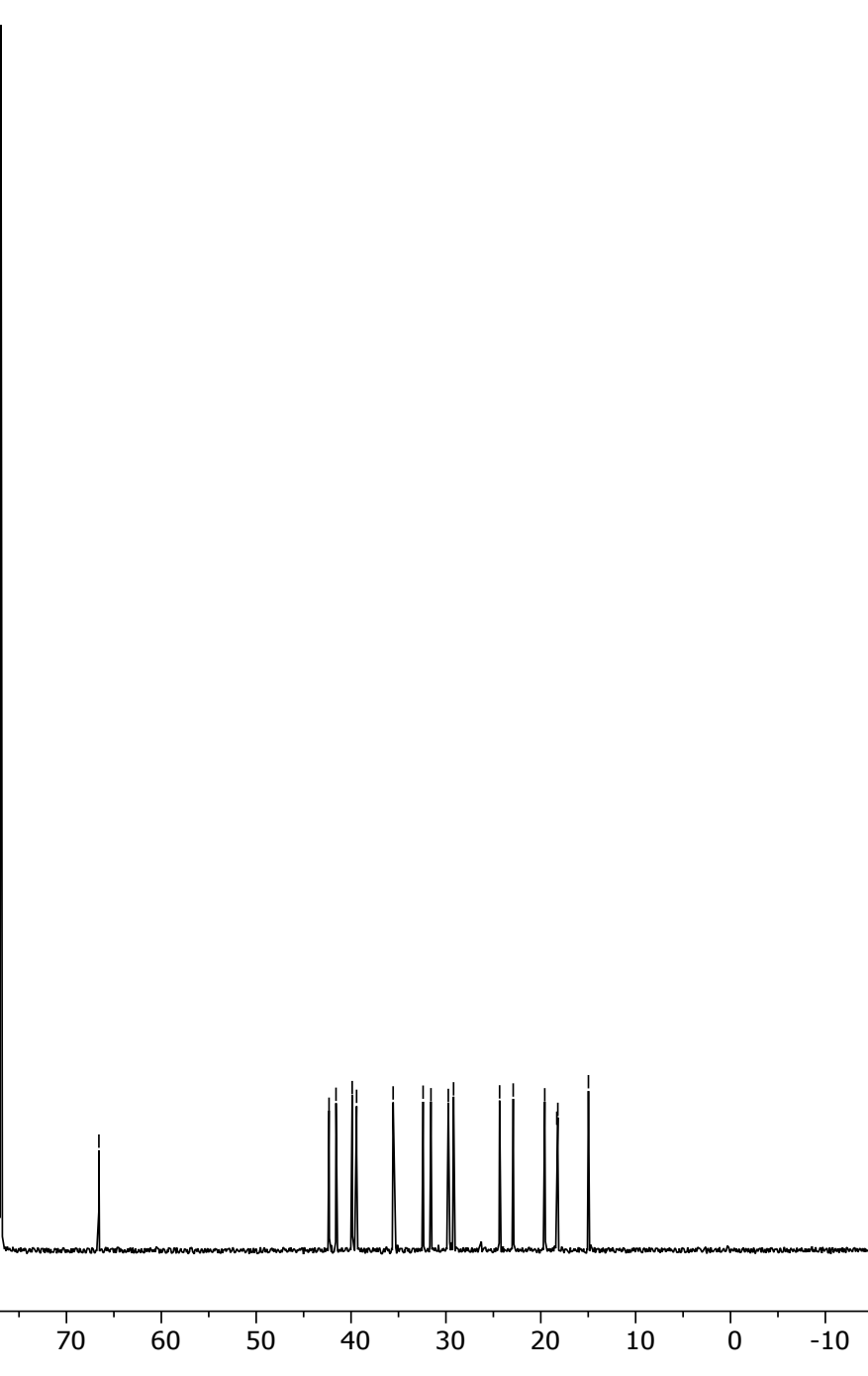




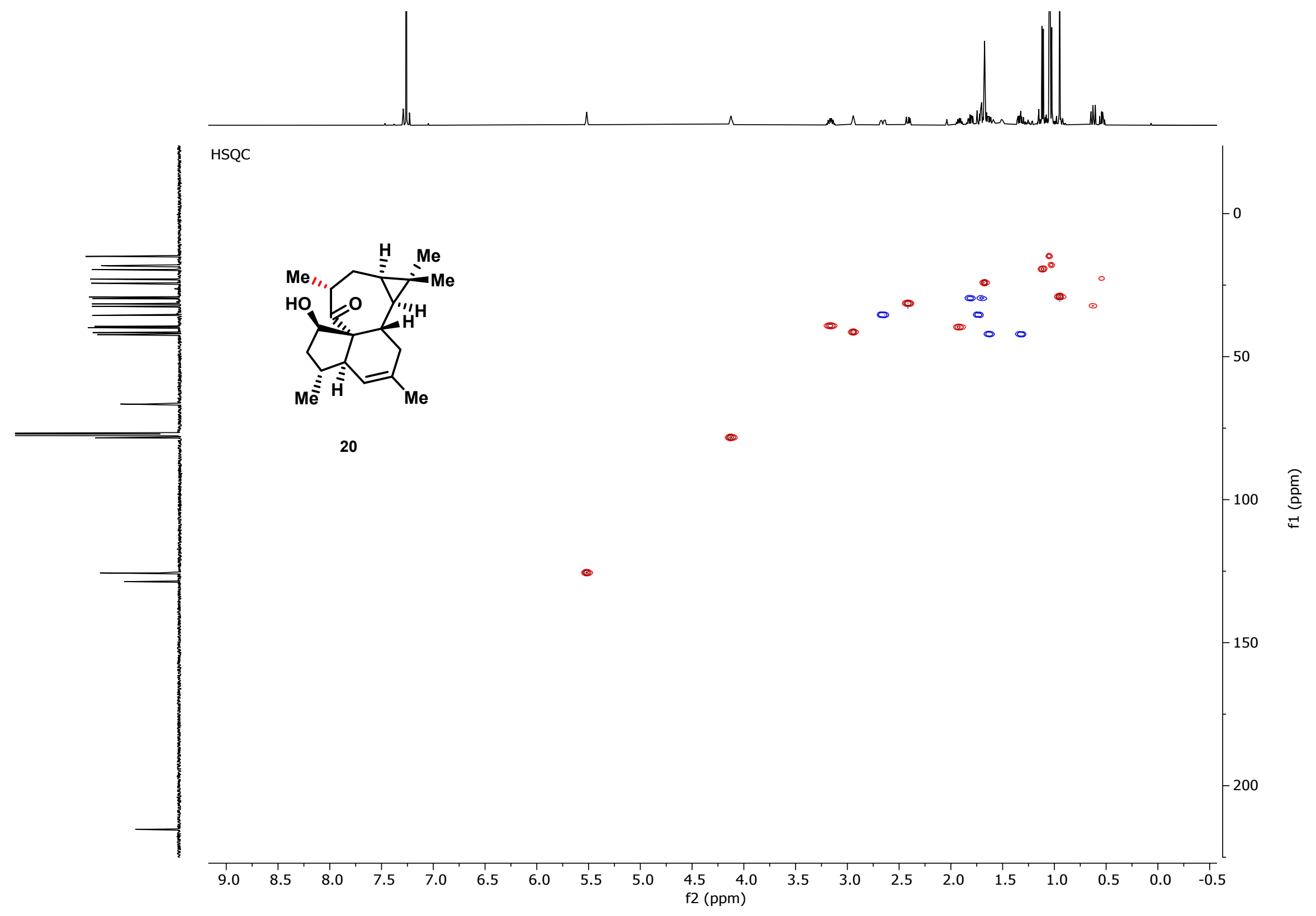




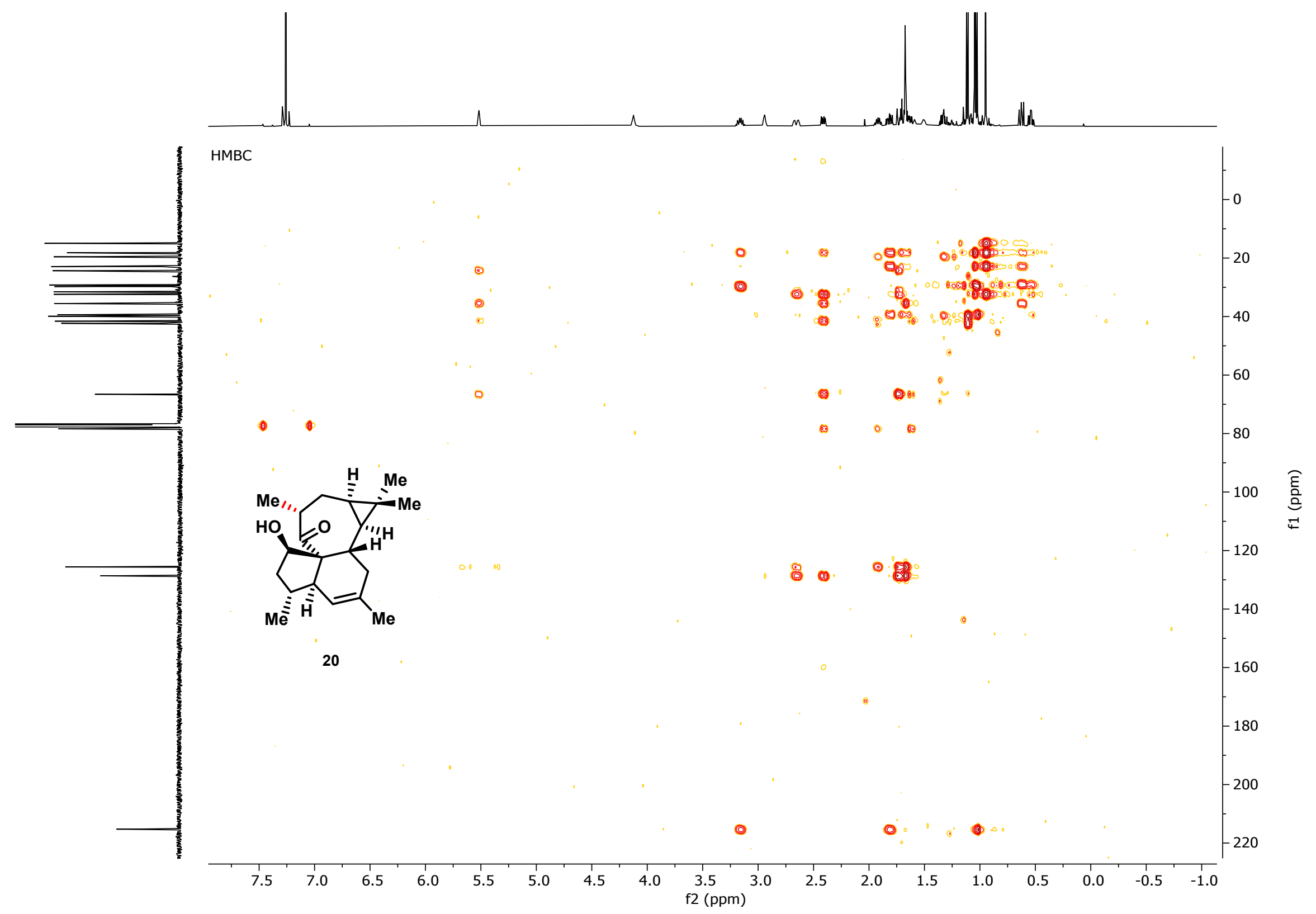




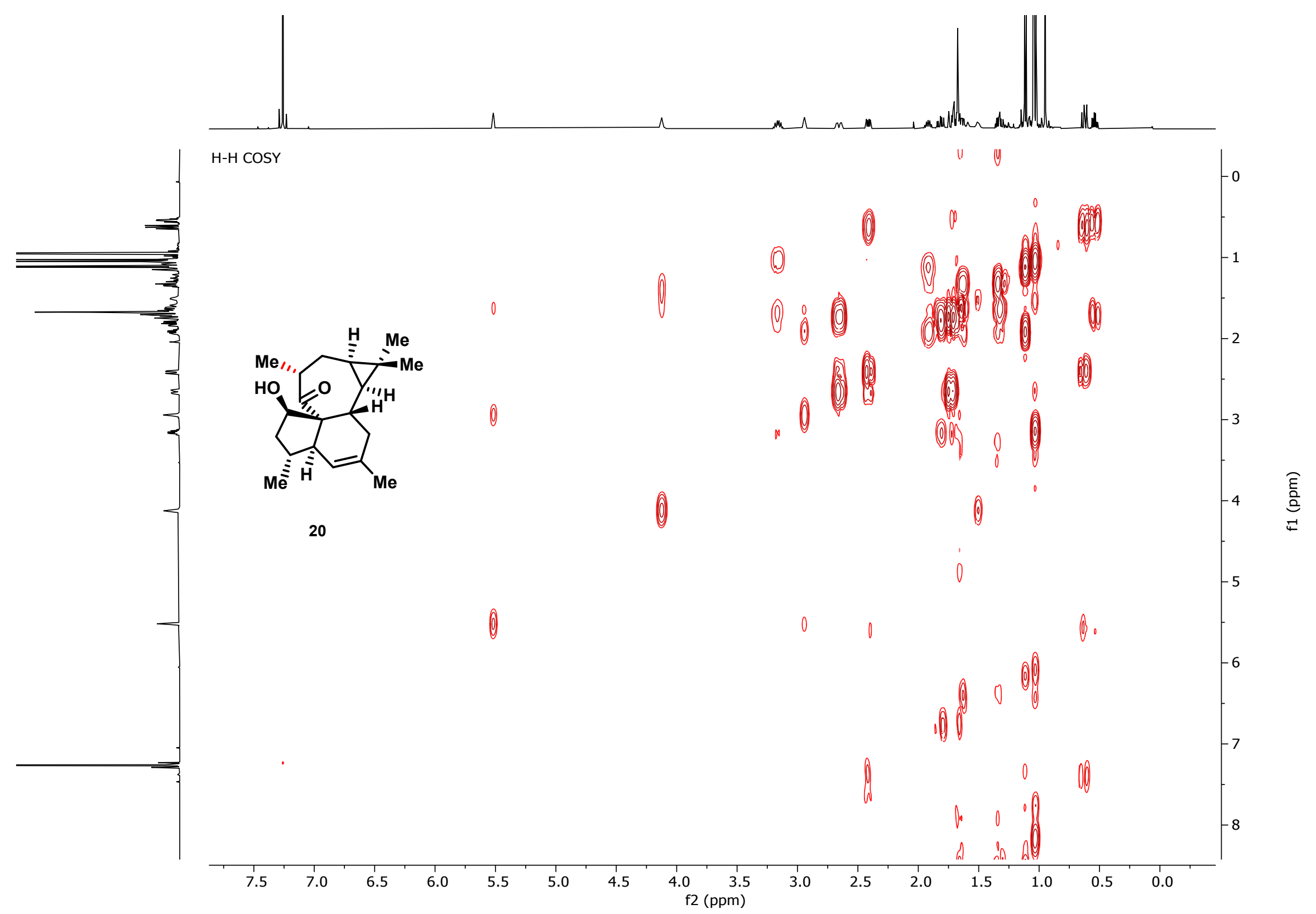




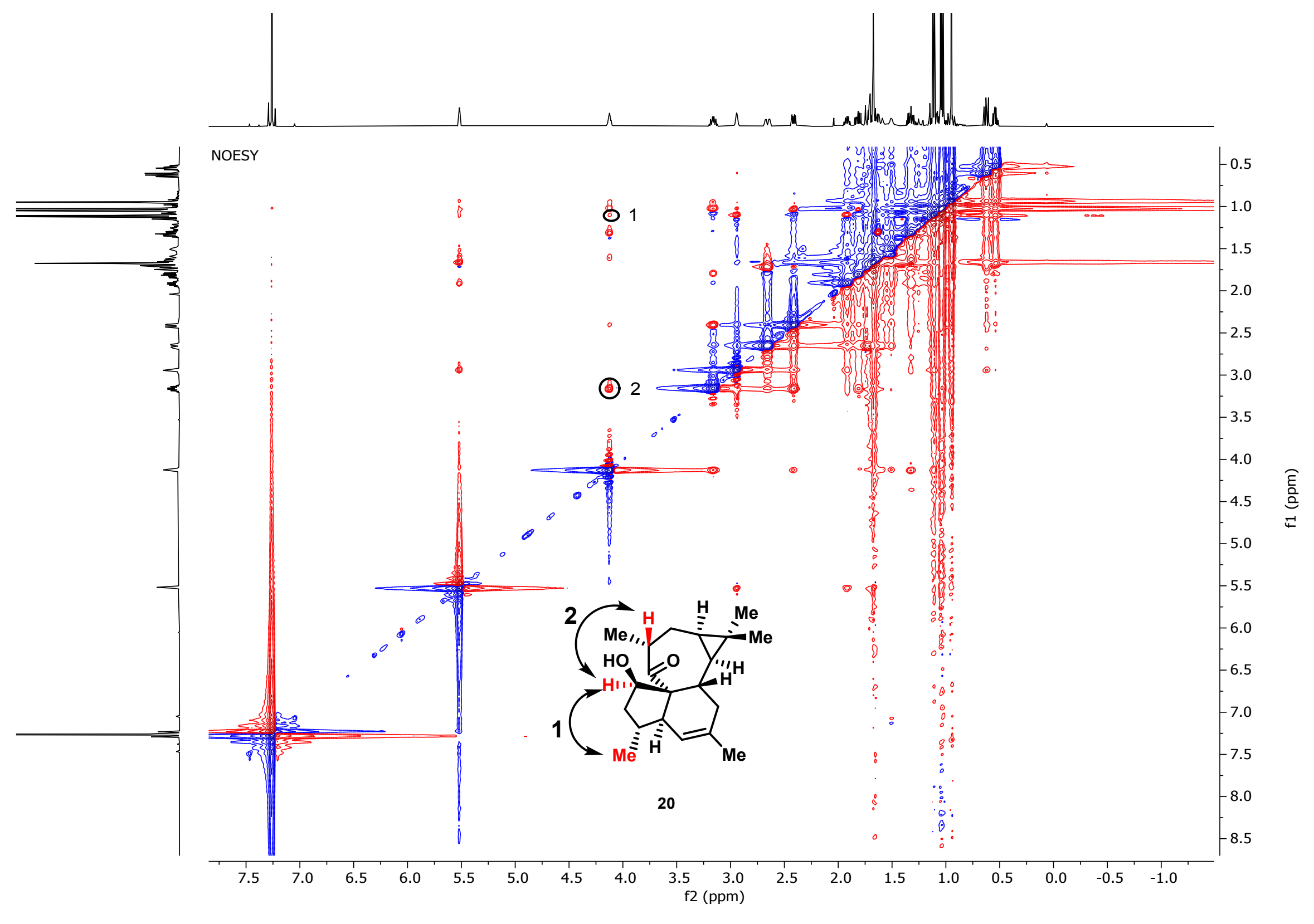



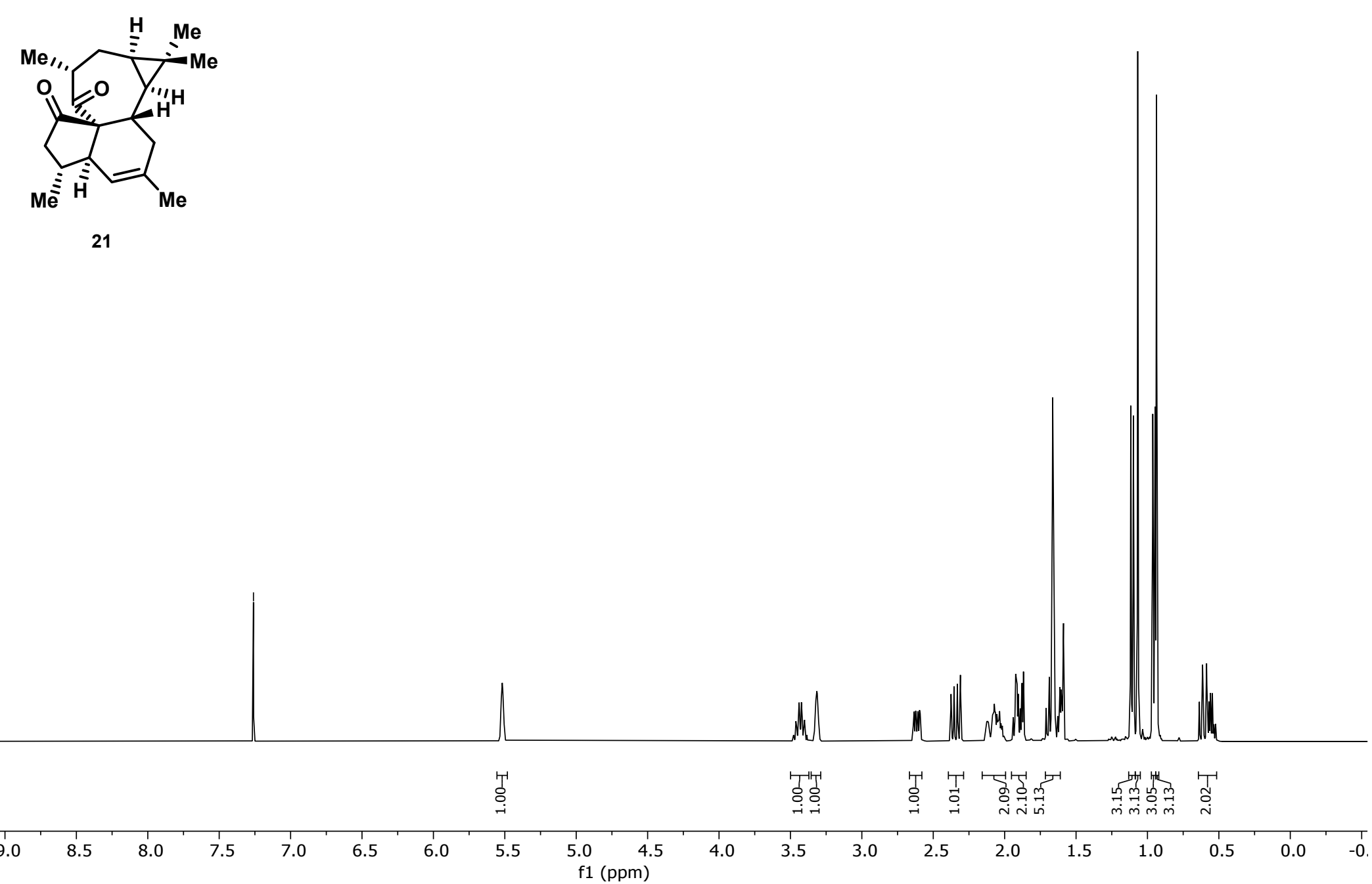

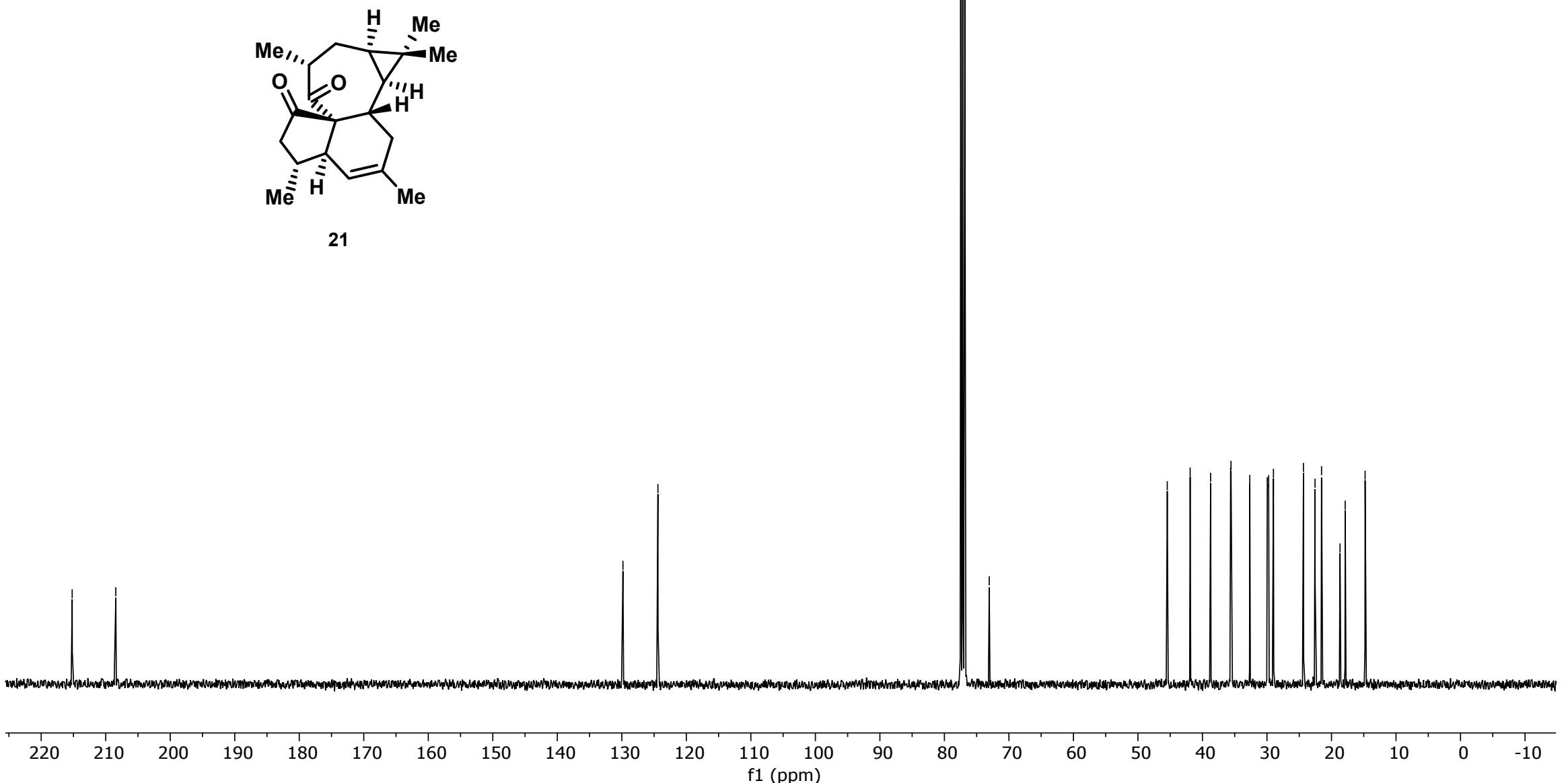

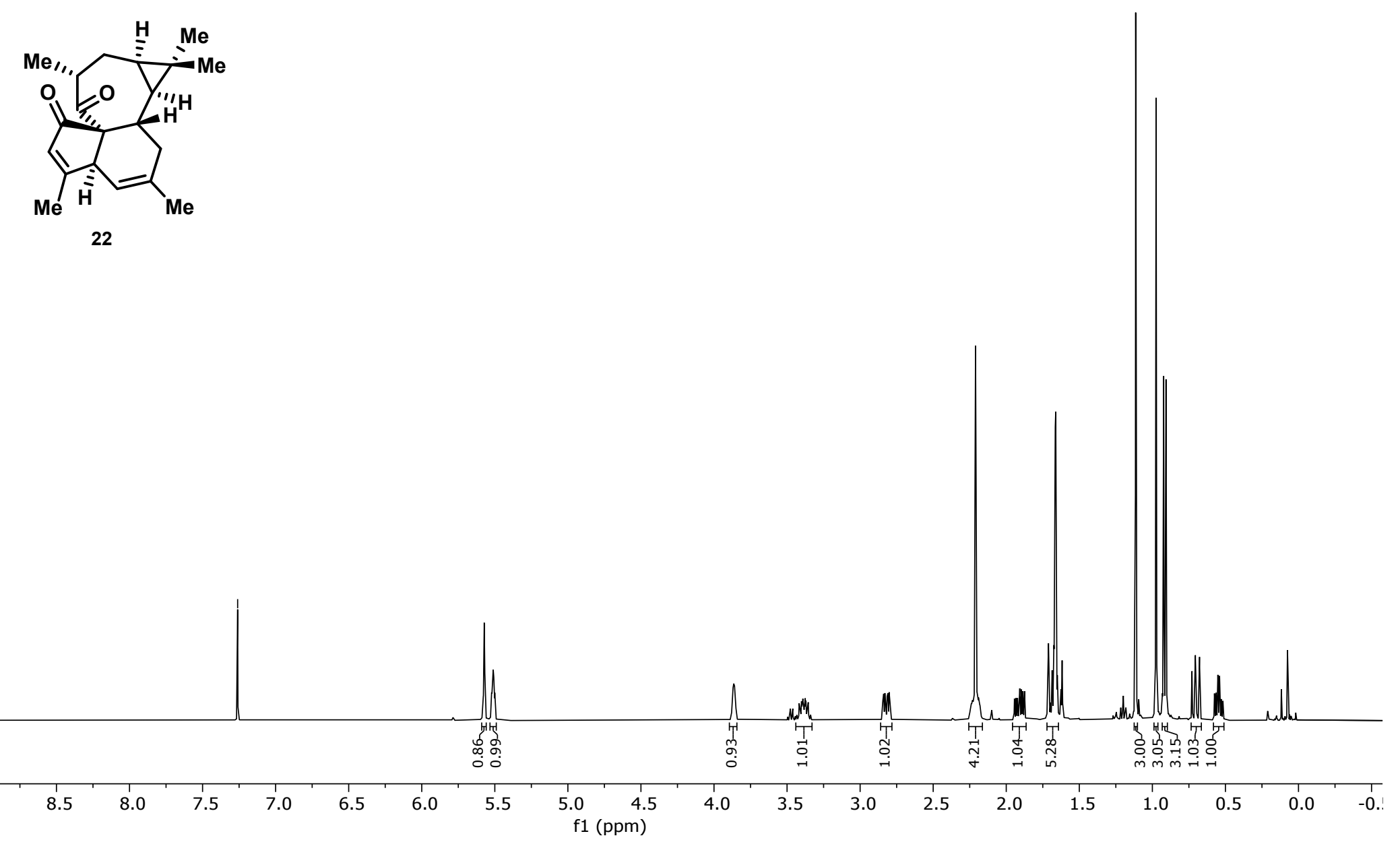


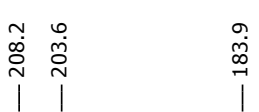

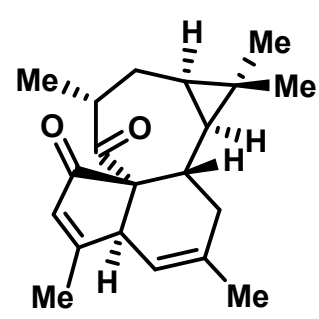

22
它

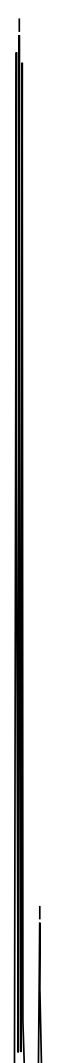

$190 \quad 180 \quad 170 \quad 160$

$\begin{array}{llll}150 & 140 & 130 & 120\end{array}$

$110 \quad 100$

$90 \quad 80$

$80 \quad 70$

60

$50 \quad 40$

1
$\quad 30 \quad 20$

10

$\begin{aligned} & 1 \\ & 0\end{aligned}-10+-20$ 

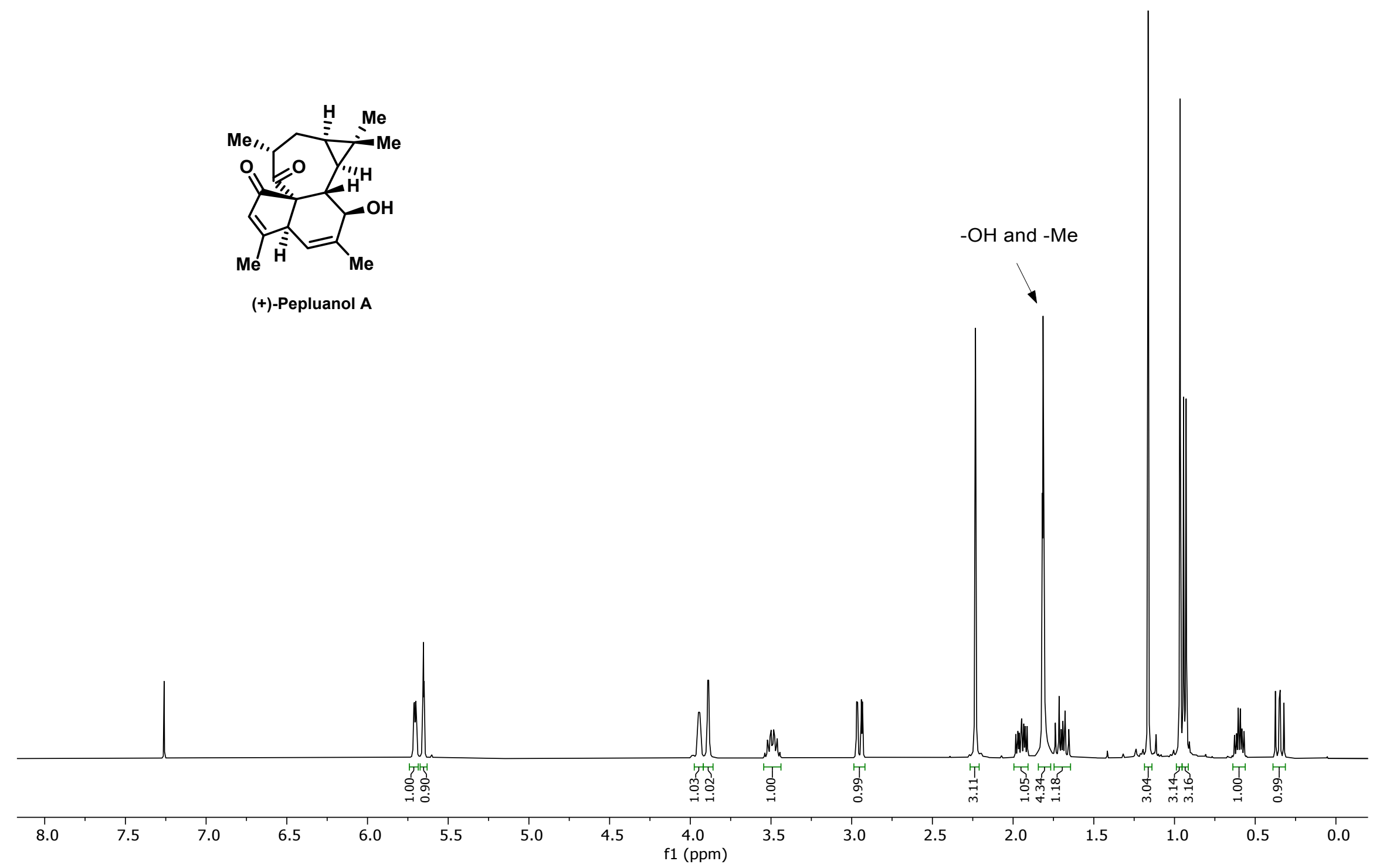

S101 

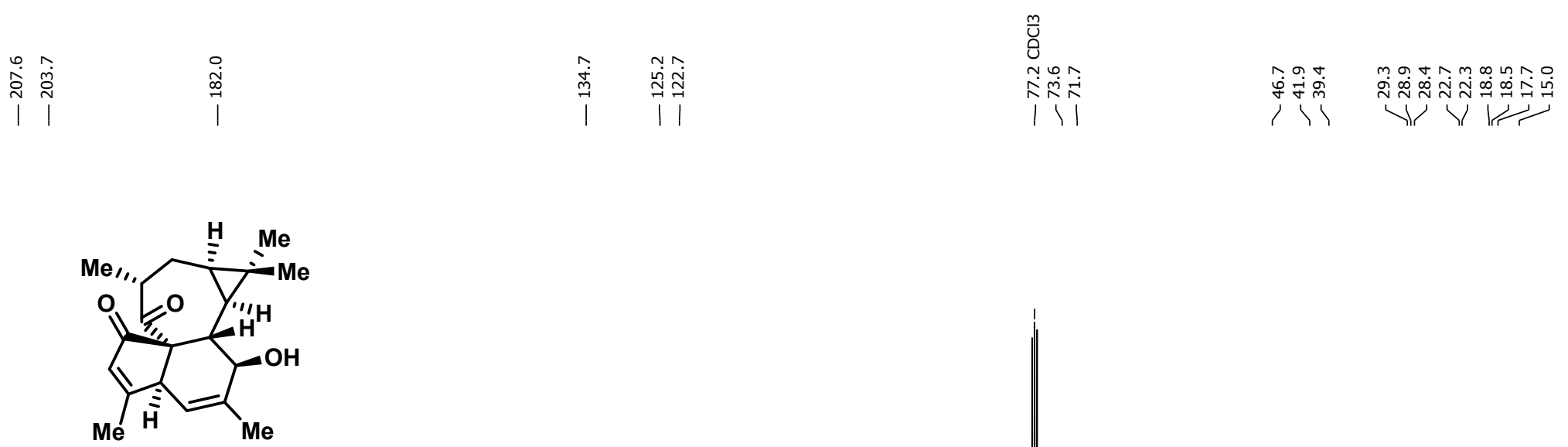

(+)-Pepluanol A

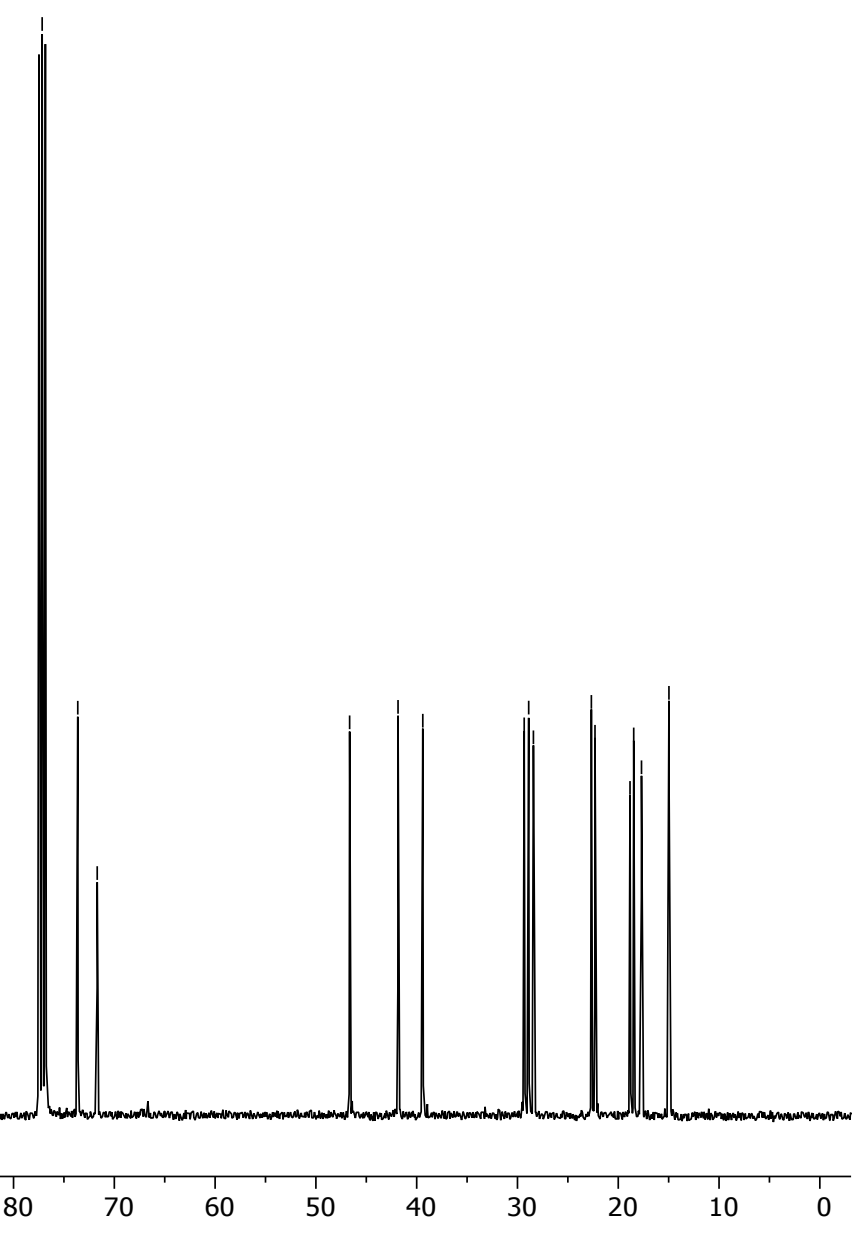




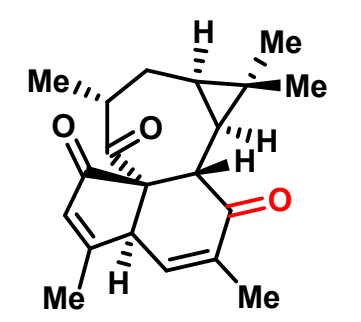

24

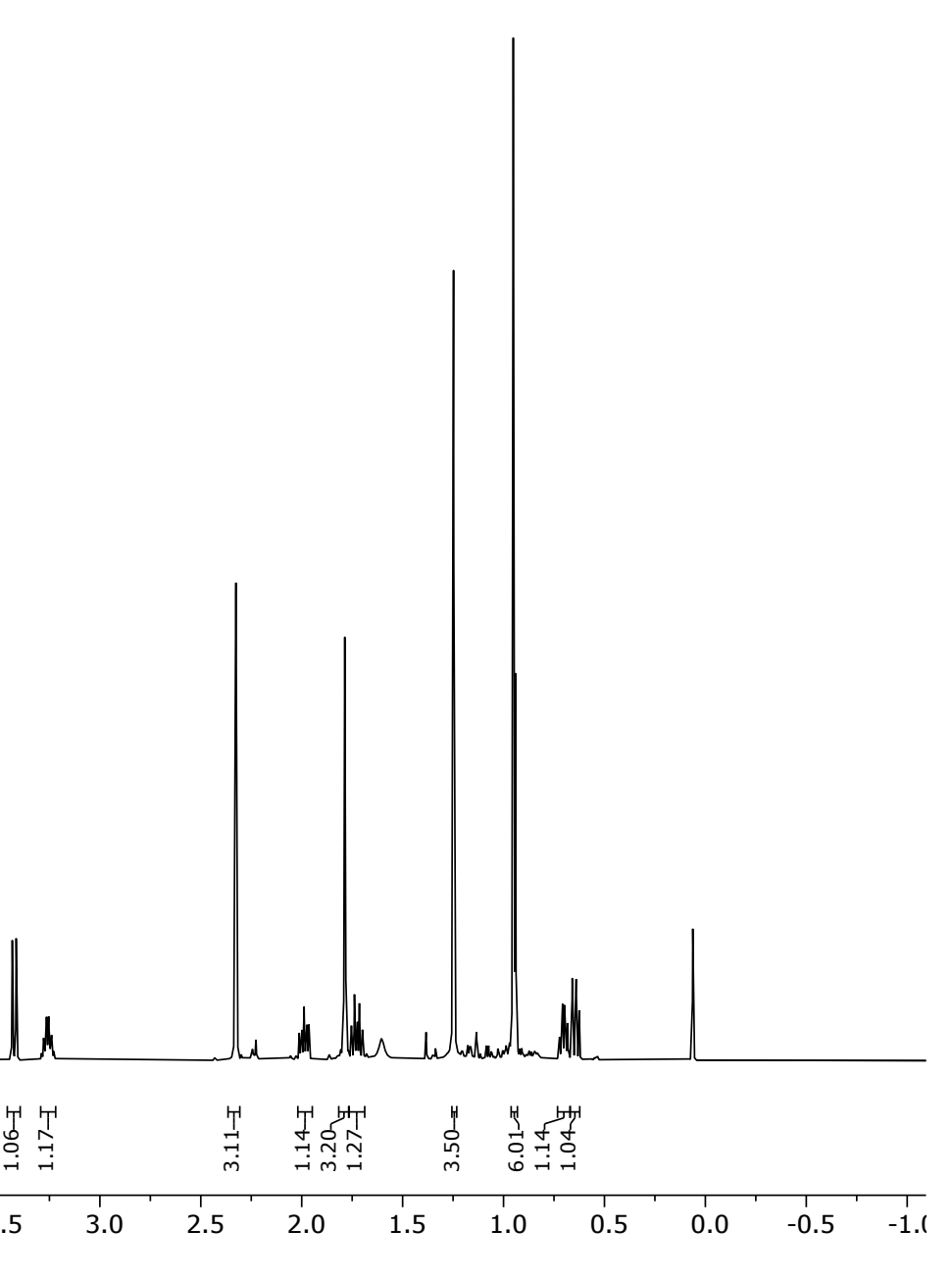




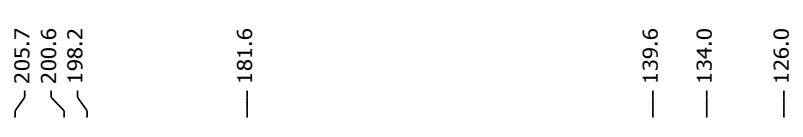

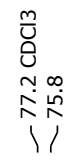

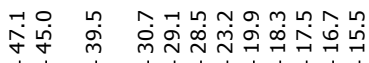

भा पर पर

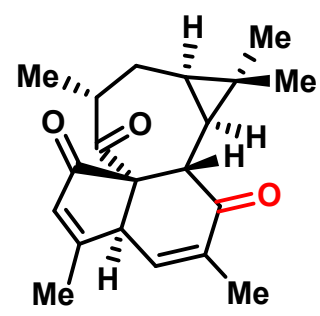

24

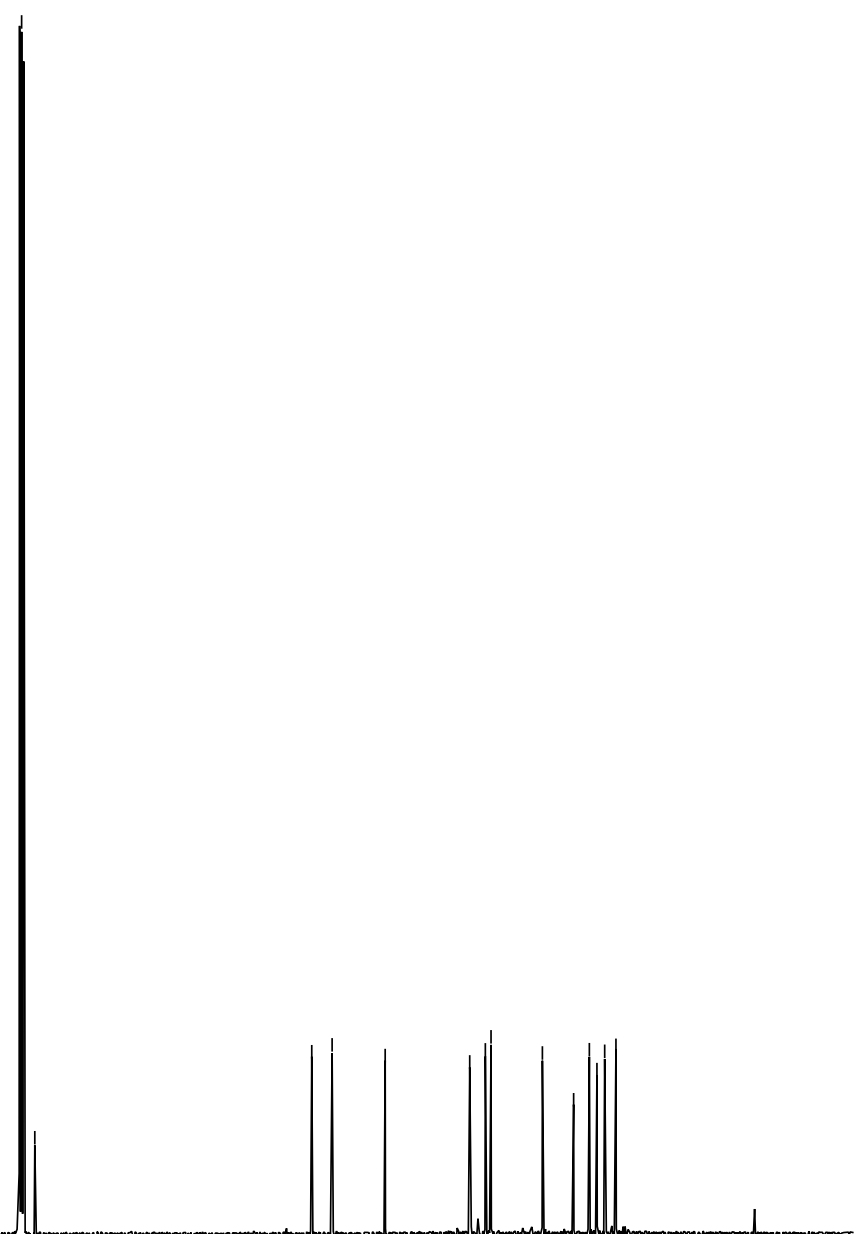

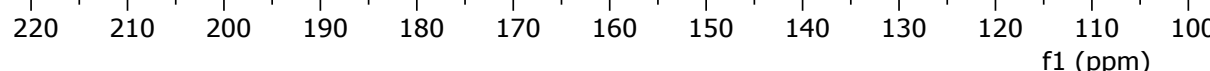

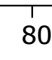

$70 \quad 60$

$50 \quad 40$

$30 \quad 20$ 


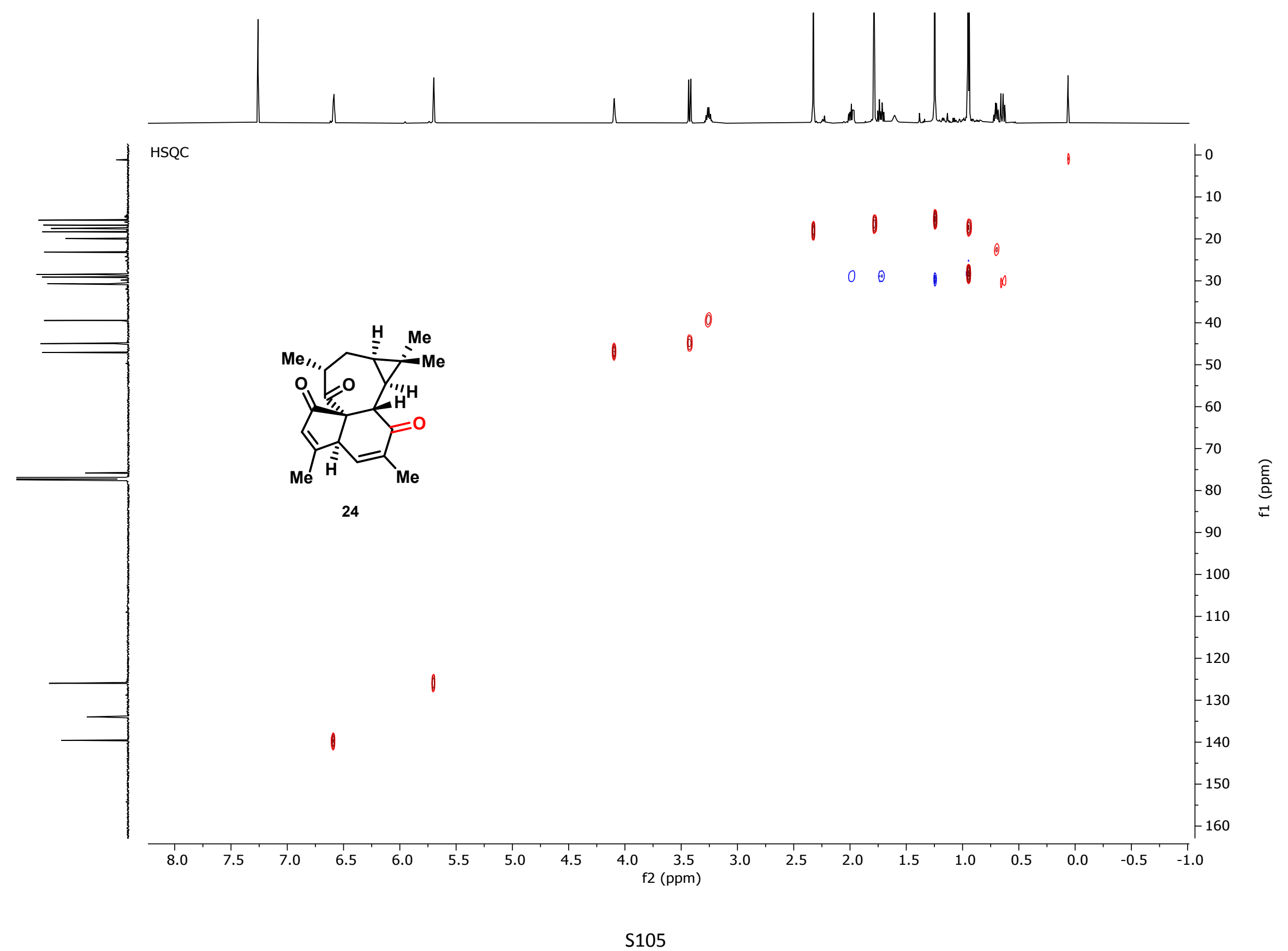




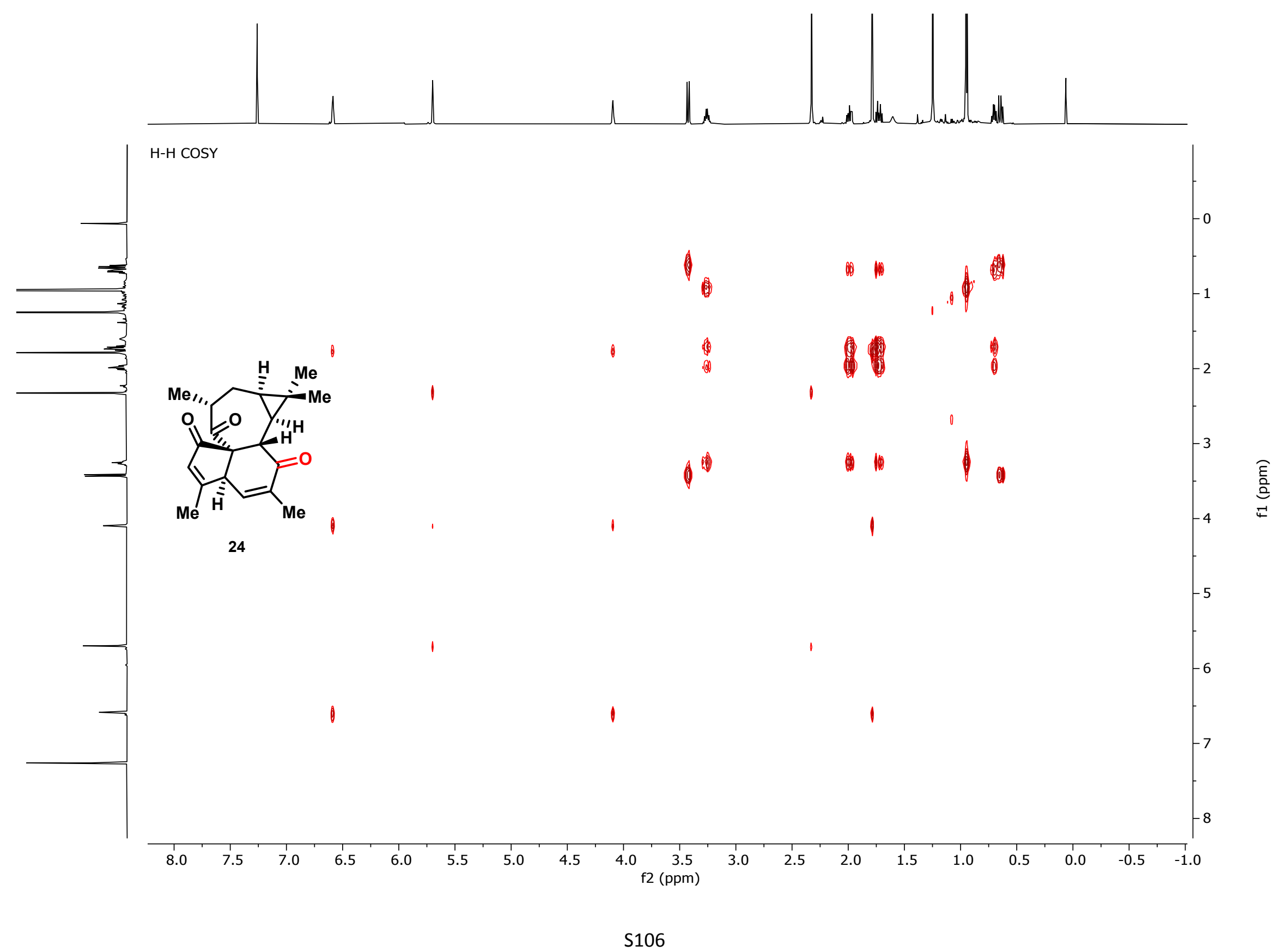




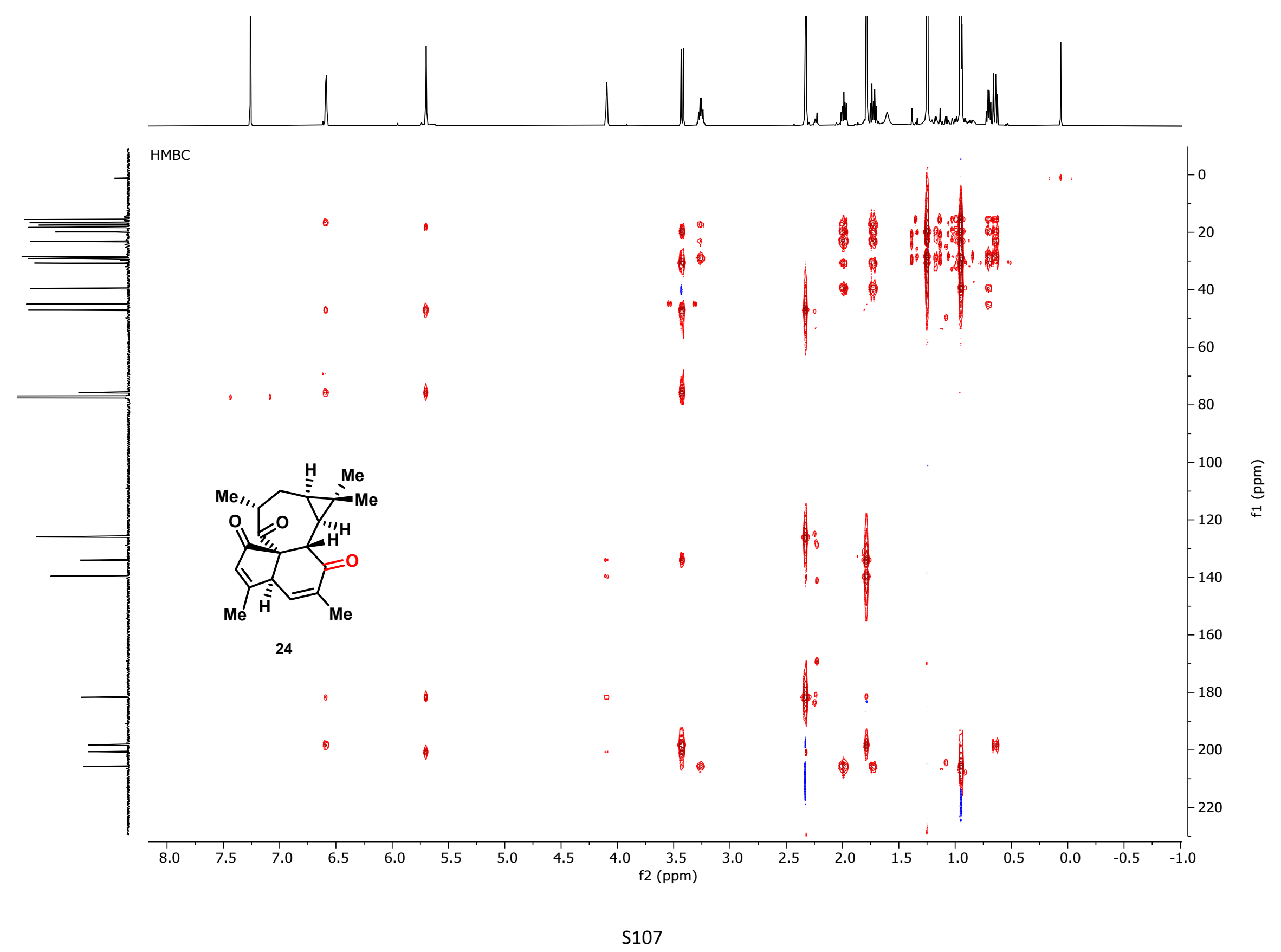




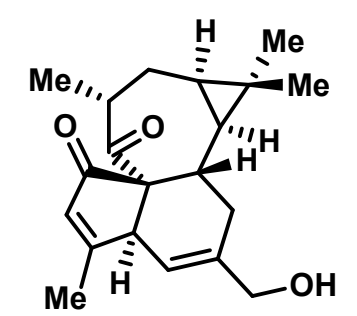

23

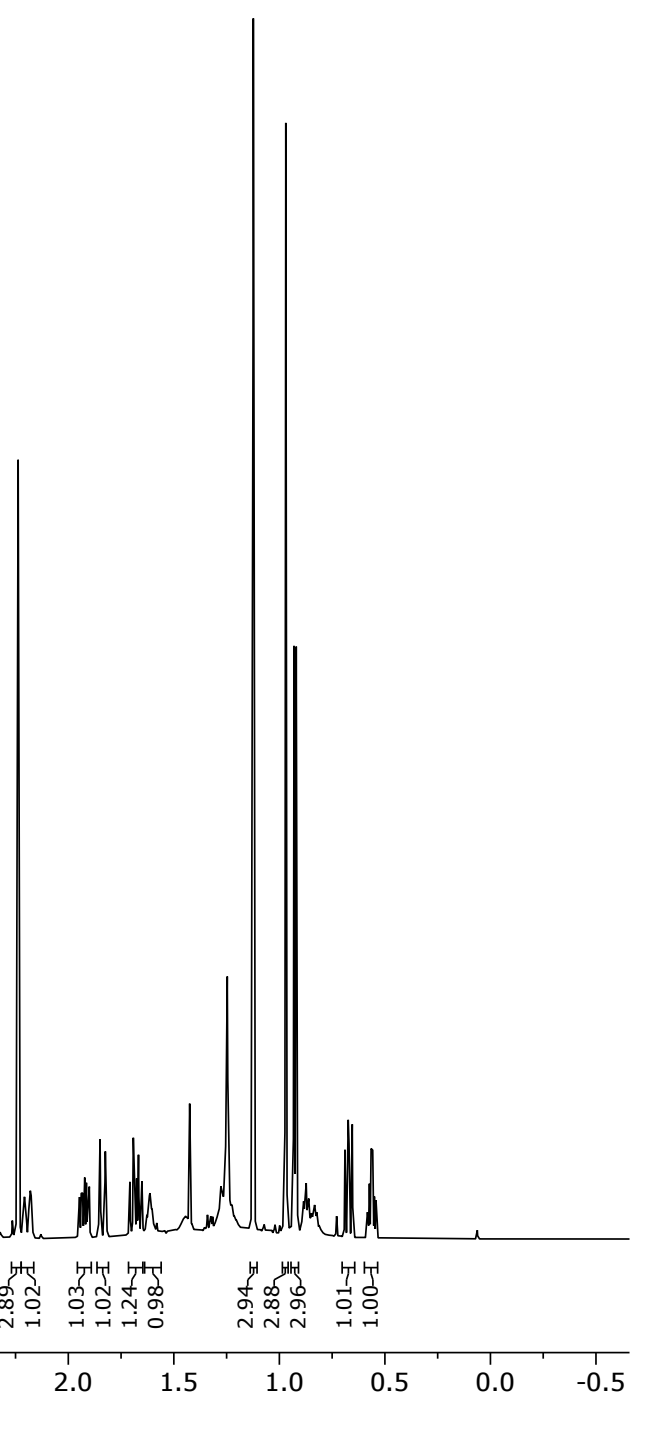




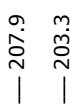

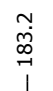

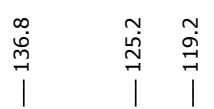

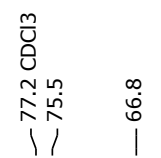

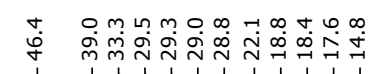
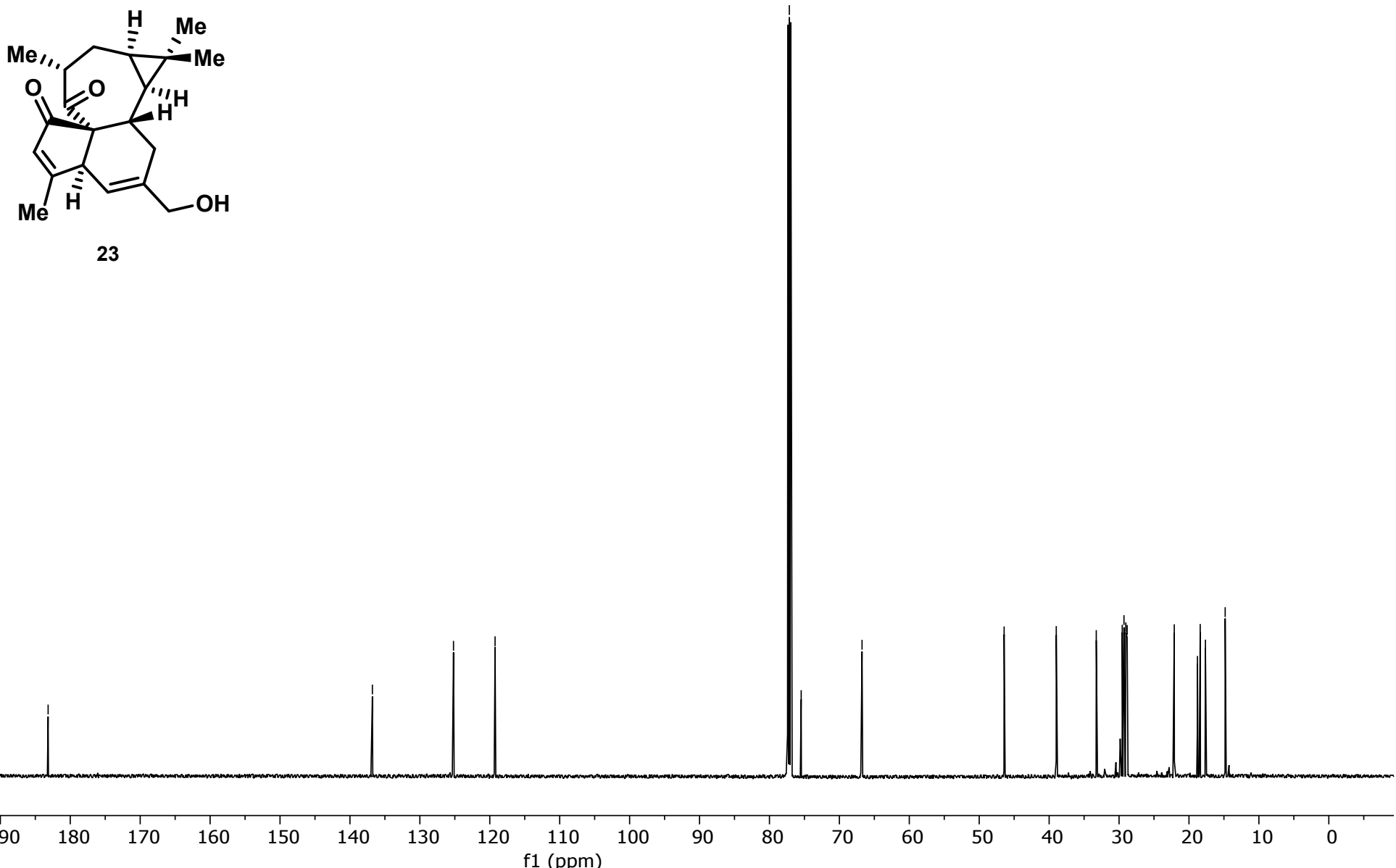

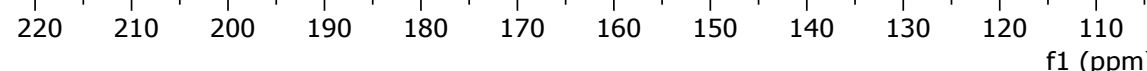




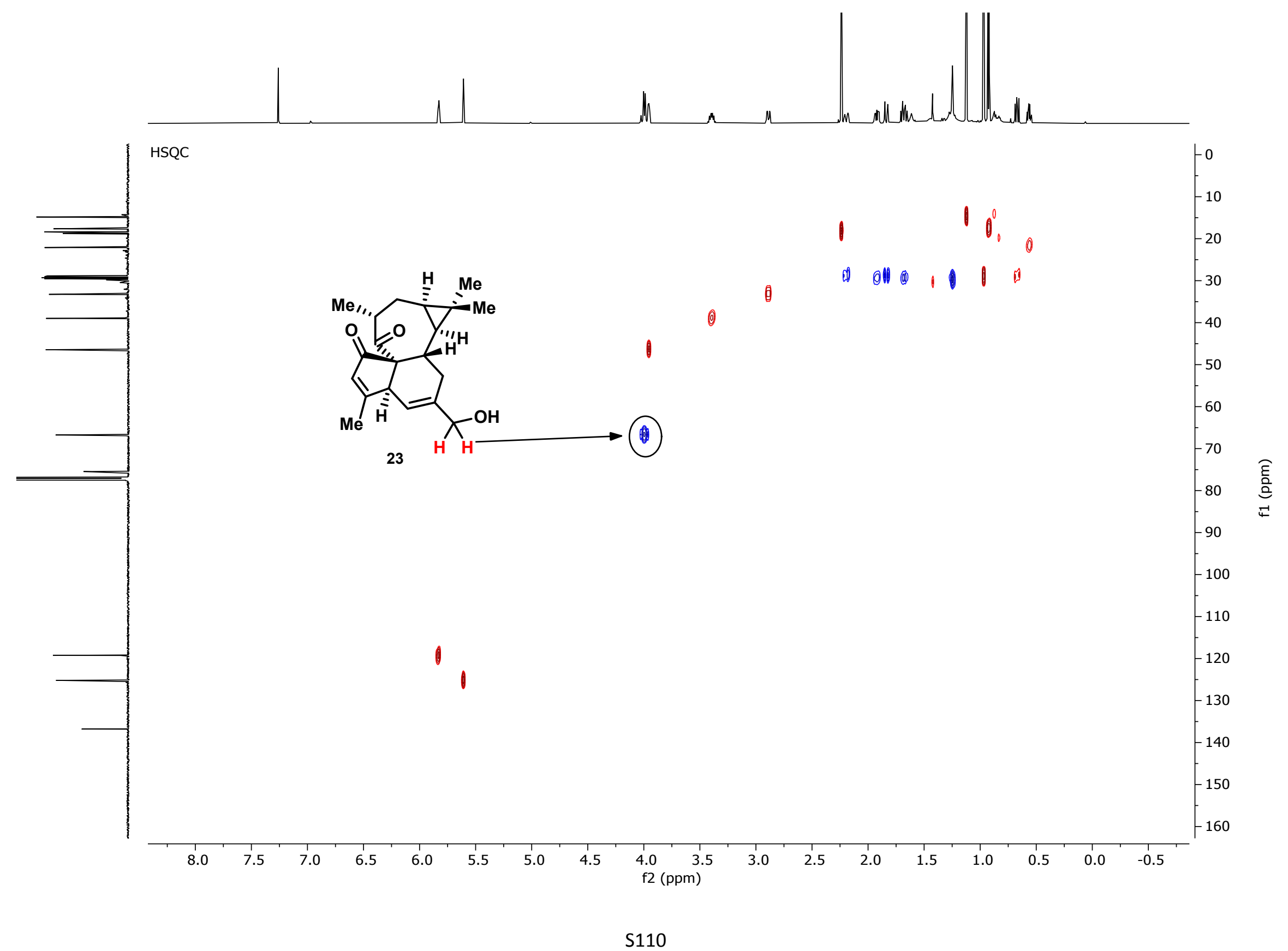




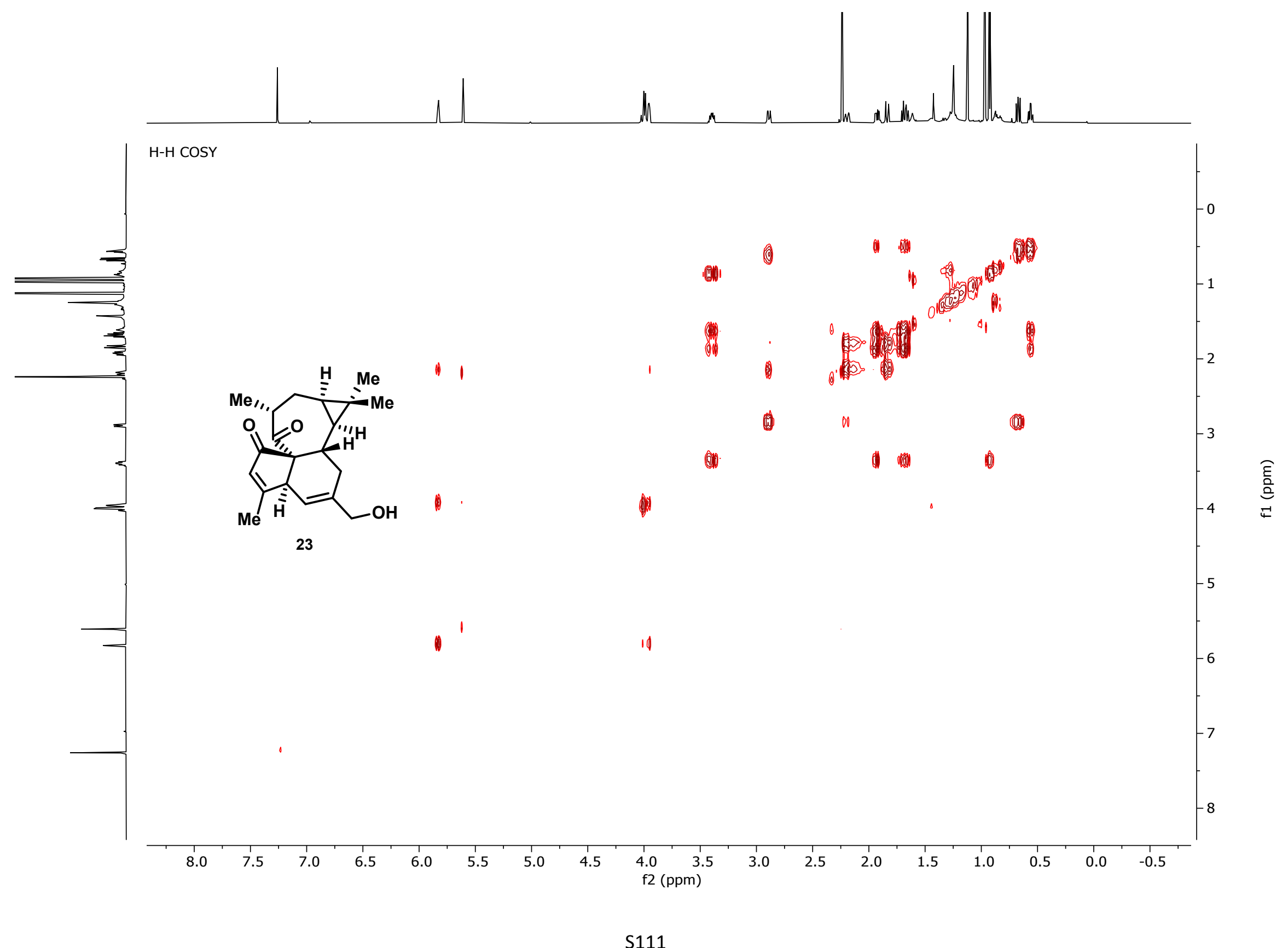




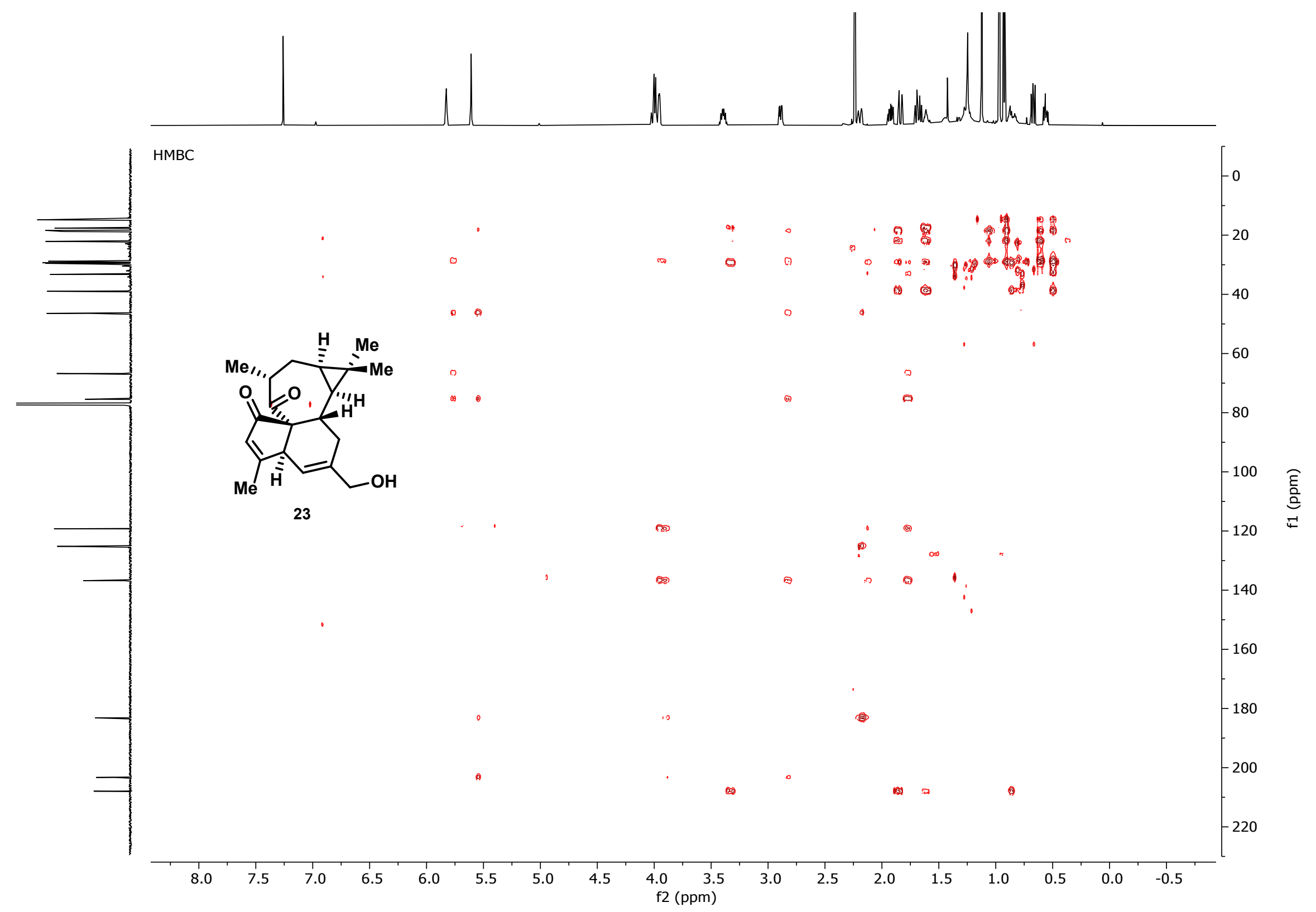




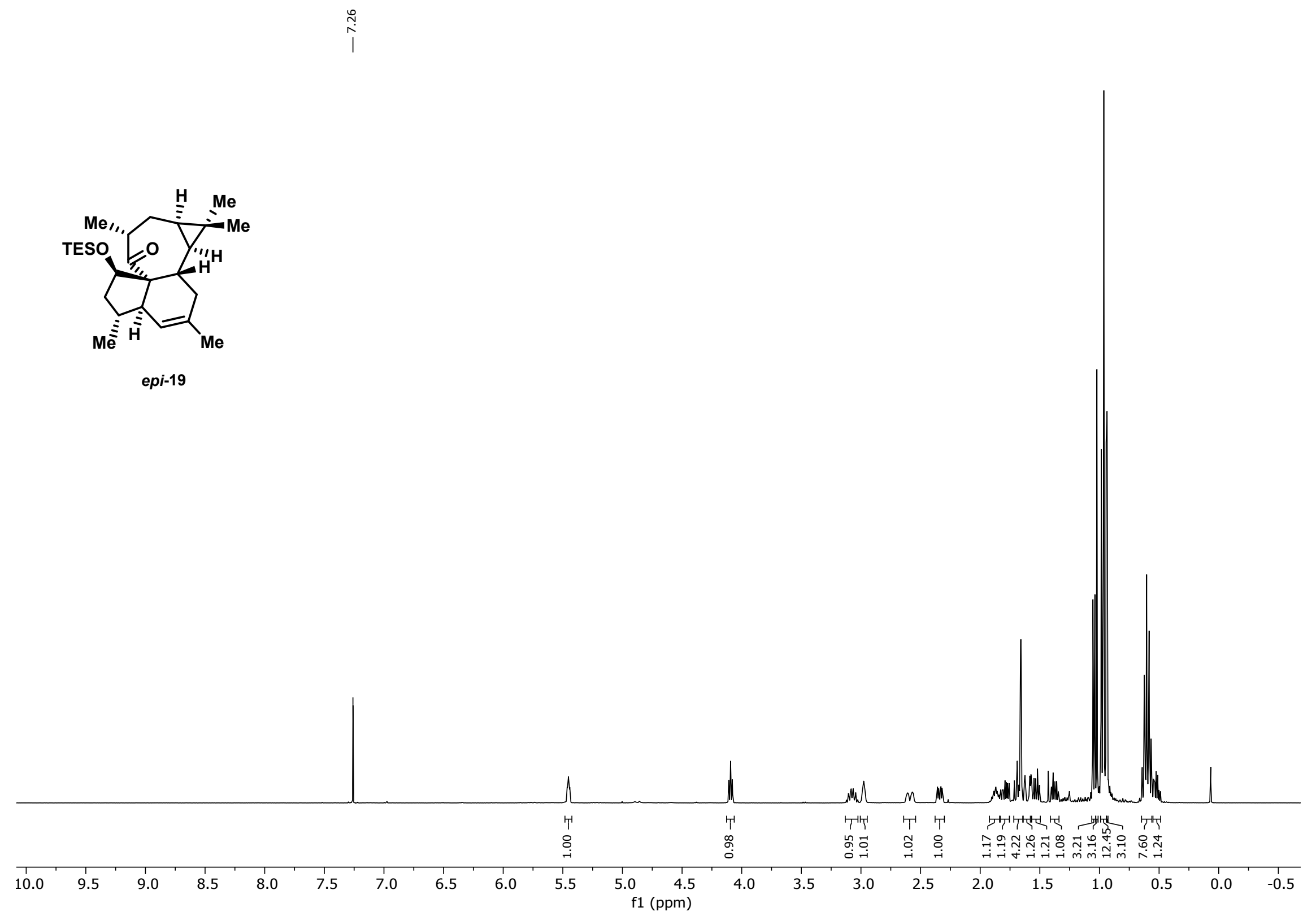



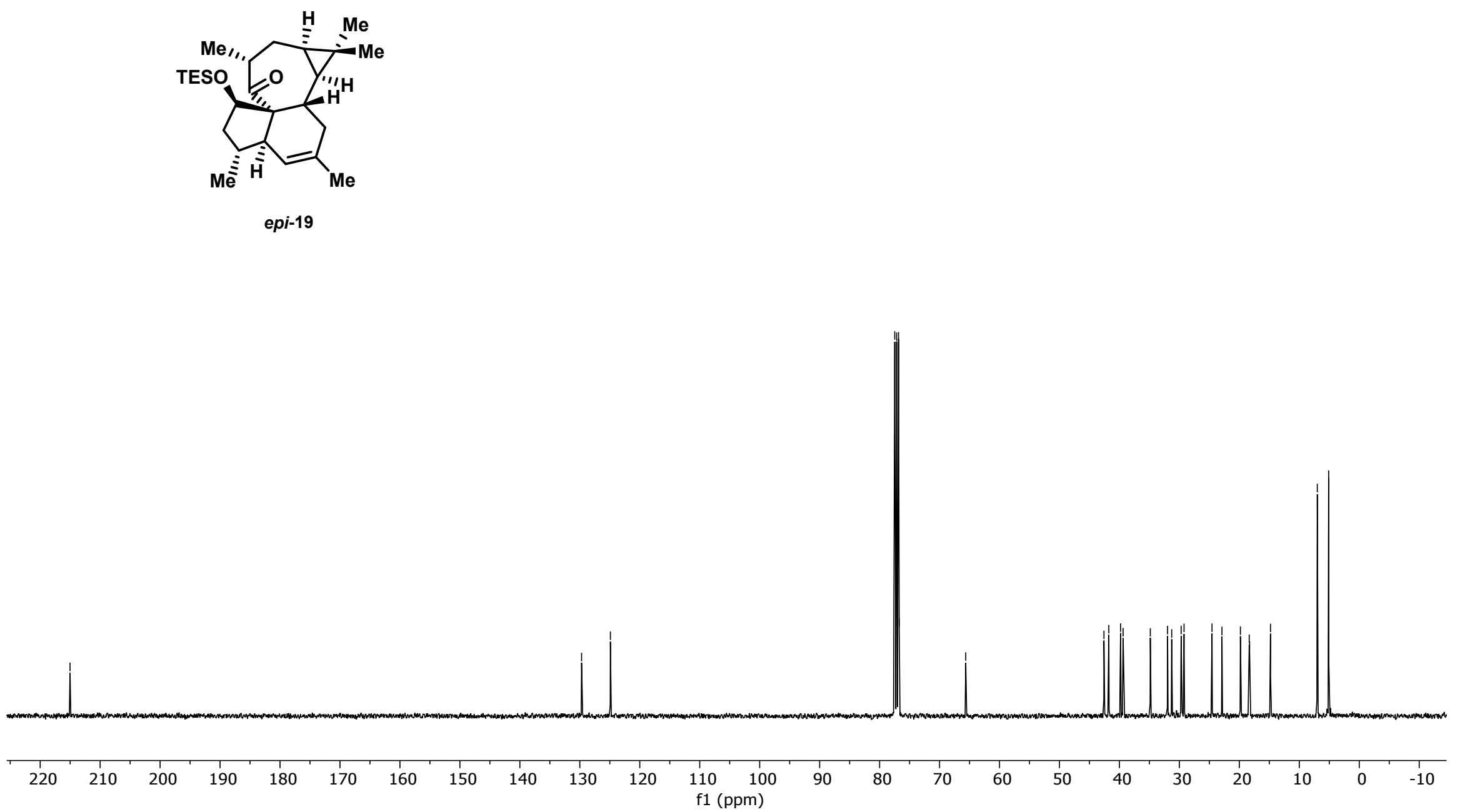


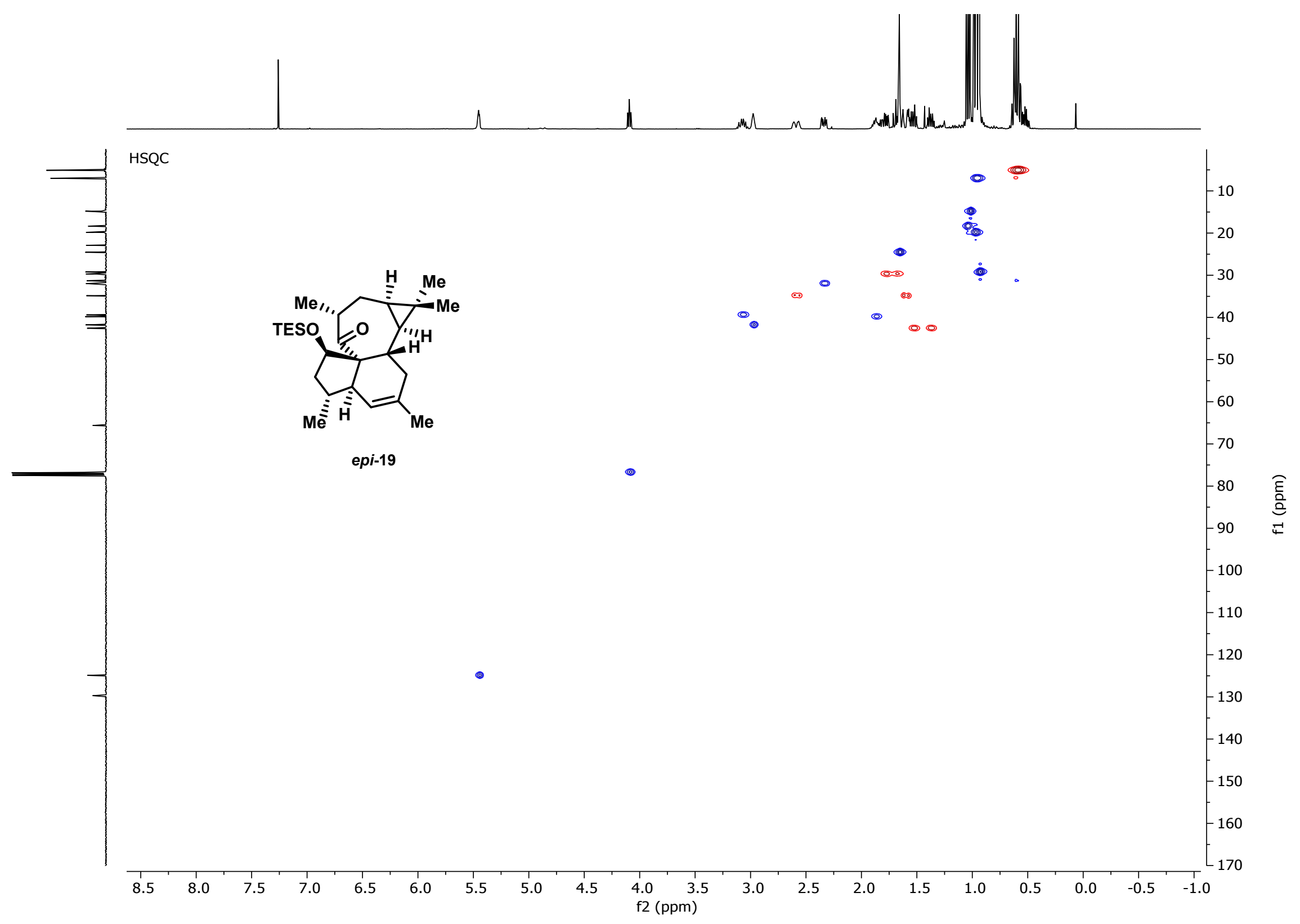




\section{DFT Calculations}

\subsection{DFT Calculations for IMDA}

The ground state electronic structures of the full models of compounds $17 \mathrm{~b}, 17 \mathrm{c}, 19$, epi-19 and the corresponding transition structures $17 \mathbf{b} \ddagger$ and $17 \mathrm{c} \ddagger$ were calculated by density functional theory (DFT) methods using the Gaussian 16 program packages. ${ }^{[5]}$ Open shell systems were calculated by the unrestricted Kohn-Sham approach (UKS). Geometry optimization followed by vibrational analysis was performed in solvent media. Solvent effects were described by the polarizable continuum model (PCM) with standard parameters for o-xylene. ${ }^{6]}$ The 6-31G(d) polarized double- $\zeta$ basis sets ${ }^{[7]}$ were employed together with the Becke Three-Parameter Hybrid Funtionals (B3LYP) ${ }^{[8]}$. To reduce computational complexity trimethylsilyl substituents were used instead of triethylsilyl substituents. Atomic coordinates of the calculated structures are provided in Tables 5.1-3-8.

Table 5.1-1: Gibbs free energies $(G)$ of compounds $17 \mathrm{~b} / \mathrm{c}, 17 \mathrm{~b} / \mathrm{c}^{\ddagger}$ and 19/epi-19.

\begin{tabular}{|c|c|}
\hline compound & Gibbs free energy (G) [hartree] \\
\hline $\mathbf{1 7 b}$ & -1338.780715 \\
\hline $\mathbf{1 7 c}$ & -1338.781284 \\
\hline $\mathbf{1 7 b} \ddagger$ & -1338.714577 \\
\hline $\mathbf{1 7} \mathbf{c}^{\ddagger}$ & -1338.729617 \\
\hline $\mathbf{1 9}$ & -1338.805688 \\
\hline epi-19 & -1338.811292 \\
\hline
\end{tabular}

Table 5.1-2: Gibbs free energy differences $(\Delta G)$.

\begin{tabular}{|c|c|c|}
\hline compounds & $\begin{array}{c}\text { Gibbs free energy } \\
\text { difference }(\Delta G) \text { [hartree] }\end{array}$ & $\begin{array}{c}\text { Gibbs free energy } \\
\text { difference }(\Delta G)[\mathrm{kcal} / \mathrm{mol}]\end{array}$ \\
\hline $17 c-17 b$ & 0.000569 & 0.357052891 \\
\hline $17 c^{\ddagger}-17 b^{\ddagger}$ & 0.015040 & 9.437742489 \\
\hline $17 b-17 b^{\ddagger}$ & 0.066138 & 41.50222159 \\
\hline $17 c-17 c^{\ddagger}$ & 0.051667 & 32.42153199 \\
\hline $19-$ epi-19 & 0.005604 & 3.516563092 \\
\hline
\end{tabular}



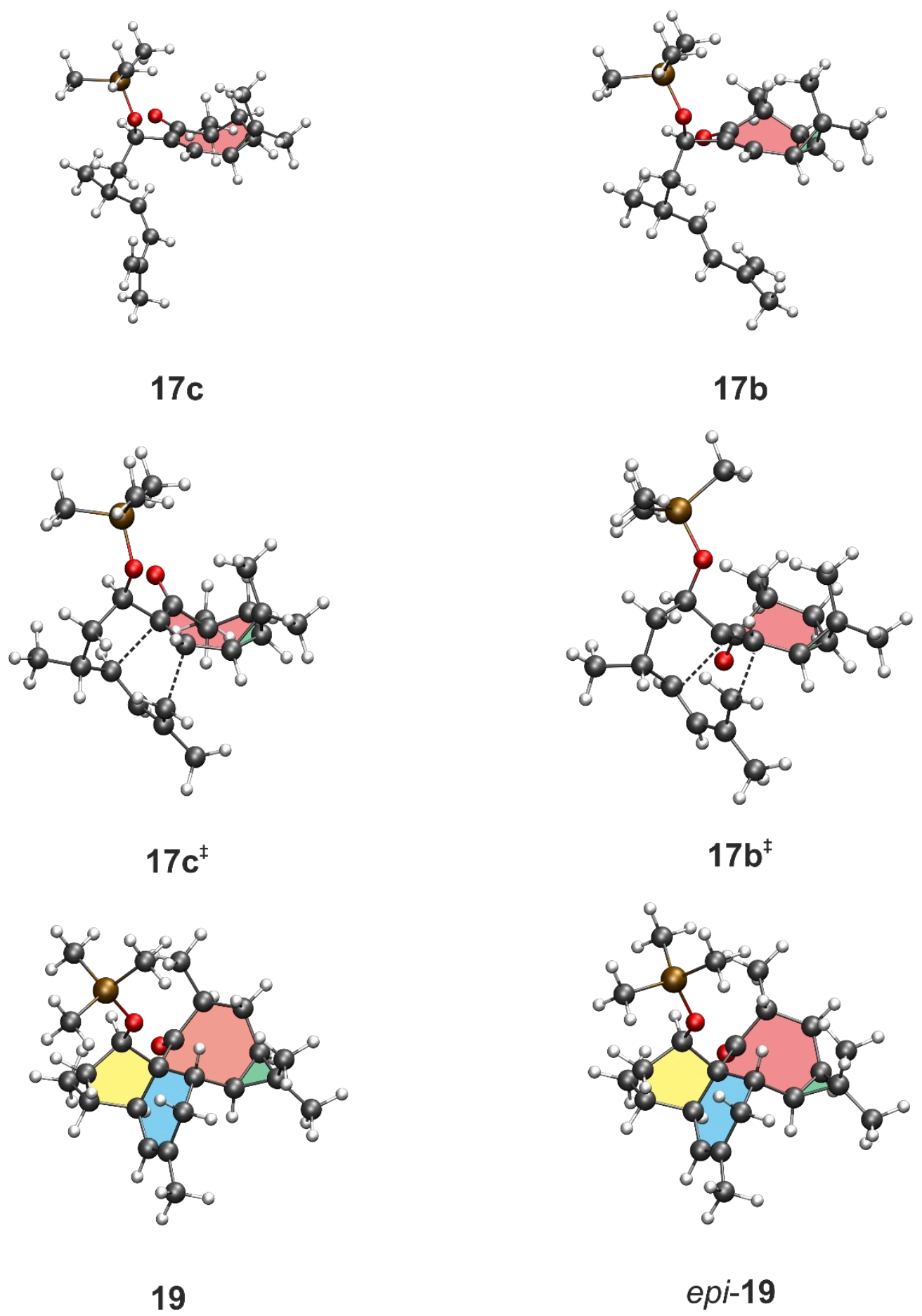

Figure 5.1-1: Calculated structures of compounds $17 \mathrm{~b} / \mathrm{c}, 17 \mathrm{~b} / \mathrm{c} \ddagger$ and 19/epi-19. 
Table 5.1-3: Atomic coordinates of structure 17c.

\begin{tabular}{|c|c|c|c|}
\hline \multicolumn{4}{|c|}{$17 c$} \\
\hline atom & $X$ & Y & Z \\
\hline C & -1.07858 & -3.4052 & -0.093 \\
\hline$C$ & -1.35917 & -2.96819 & 1.33387 \\
\hline C & -0.16454 & -2.19192 & 1.95222 \\
\hline C & -0.25122 & -0.69813 & 1.61518 \\
\hline C & -0.26302 & -0.21178 & 0.20172 \\
\hline C & -0.39486 & -0.97 & -0.90967 \\
\hline C & -0.56435 & -2.42379 & -1.11978 \\
\hline $\mathrm{O}$ & -0.29611 & 0.12 & 2.53416 \\
\hline C & -1.95335 & -3.07084 & -1.28628 \\
\hline $\mathrm{H}$ & 0.17221 & -2.81213 & -1.82512 \\
\hline $\mathrm{H}$ & -0.59921 & -4.38254 & -0.14689 \\
\hline C & -2.0848 & -4.1291 & -2.36928 \\
\hline C & -3.20309 & -2.22533 & -1.11618 \\
\hline C & -0.06508 & -2.40474 & 3.46621 \\
\hline $\mathrm{H}$ & -2.26833 & -2.36116 & 1.40672 \\
\hline $\mathrm{H}$ & -1.54818 & -3.86008 & 1.94323 \\
\hline $\mathrm{H}$ & 0.75305 & -2.57152 & 1.4792 \\
\hline $\mathrm{H}$ & -2.91015 & -4.81672 & -2.14238 \\
\hline $\mathrm{H}$ & -2.29309 & -3.67196 & -3.34507 \\
\hline $\mathrm{H}$ & -1.17033 & -4.72503 & -2.46572 \\
\hline $\mathrm{H}$ & -4.03952 & -2.84002 & -0.75938 \\
\hline $\mathrm{H}$ & -3.06424 & -1.39949 & -0.41418 \\
\hline $\mathrm{H}$ & -3.50057 & -1.78939 & -2.07796 \\
\hline $\mathrm{H}$ & 0.08023 & -3.46905 & 3.6823 \\
\hline $\mathrm{H}$ & -0.97698 & -2.07194 & 3.97278 \\
\hline $\mathrm{H}$ & 0.77038 & -1.84526 & 3.89602 \\
\hline C & 4.46368 & 0.57925 & -2.19051 \\
\hline $\mathrm{H}$ & 5.17625 & 0.83713 & -2.97033 \\
\hline $\mathrm{H}$ & 3.42599 & 0.83537 & -2.37728 \\
\hline C & 2.93428 & 0.27312 & 0.57667 \\
\hline $\mathrm{H}$ & 2.42038 & -0.19398 & 1.41664 \\
\hline
\end{tabular}

\begin{tabular}{|c|c|c|c|}
\hline atom & $x$ & $Y$ & Z \\
\hline C & 4.85488 & -0.05219 & -1.07164 \\
\hline C & 3.94133 & -0.42979 & 0.02704 \\
\hline $\mathrm{H}$ & 4.16895 & -1.39579 & 0.48278 \\
\hline $\mathrm{H}$ & -0.38798 & -0.41011 & -1.84655 \\
\hline C & 6.29657 & -0.47002 & -0.87274 \\
\hline $\mathrm{H}$ & 6.9176 & -0.20186 & -1.73277 \\
\hline $\mathrm{H}$ & 6.72544 & -0.00052 & 0.02208 \\
\hline $\mathrm{H}$ & 6.37137 & -1.55568 & -0.72261 \\
\hline C & 2.48312 & 1.67778 & 0.25528 \\
\hline $\mathrm{H}$ & 3.26158 & 2.13811 & -0.36591 \\
\hline C & -0.14706 & 1.30699 & 0.02183 \\
\hline $\mathrm{H}$ & -0.26068 & 1.76211 & 1.00866 \\
\hline C & 1.18719 & 1.77128 & -0.59725 \\
\hline $\mathrm{H}$ & 1.04819 & 2.82848 & -0.85735 \\
\hline $\mathrm{H}$ & 1.33038 & 1.23351 & -1.54313 \\
\hline C & 2.3723 & 2.49961 & 1.55627 \\
\hline $\mathrm{H}$ & 3.34505 & 2.56125 & 2.05676 \\
\hline $\mathrm{H}$ & 2.03656 & 3.52212 & 1.34437 \\
\hline $\mathrm{H}$ & 1.66373 & 2.04823 & 2.25941 \\
\hline $\mathrm{O}$ & -1.18643 & 1.7715 & -0.85962 \\
\hline $\mathrm{Si}$ & -2.51104 & 2.66698 & -0.3341 \\
\hline C & -3.55344 & 2.93504 & -1.87774 \\
\hline $\mathrm{H}$ & -4.45175 & 3.52127 & -1.64813 \\
\hline $\mathrm{H}$ & -2.99009 & 3.47489 & -2.64764 \\
\hline $\mathrm{H}$ & -3.87794 & 1.98041 & -2.30766 \\
\hline $\mathrm{C}$ & -3.47736 & 1.71666 & 0.98149 \\
\hline $\mathrm{H}$ & -3.92846 & 0.80853 & 0.56378 \\
\hline $\mathrm{H}$ & -2.82967 & 1.416 & 1.81369 \\
\hline $\mathrm{H}$ & -4.28715 & 2.33059 & 1.39545 \\
\hline C & -1.93953 & 4.32236 & 0.37587 \\
\hline $\mathrm{H}$ & -1.28224 & 4.19114 & 1.2439 \\
\hline $\mathrm{H}$ & -1.39159 & 4.90696 & -0.3727 \\
\hline $\mathrm{H}$ & -2.79783 & 4.9219 & 0.70466 \\
\hline
\end{tabular}


Table 5.1-4: Atomic coordinates of structure 17c ${ }^{\ddagger}$.

\begin{tabular}{|c|c|c|c|}
\hline \multicolumn{4}{|c|}{$17 c^{\ddagger}$} \\
\hline atom & $X$ & Y & Z \\
\hline C & 2.2741 & -2.31636 & 0.14522 \\
\hline C & 1.97385 & -1.98479 & 1.60161 \\
\hline C & 1.89516 & -0.464 & 1.88507 \\
\hline C & 0.50624 & 0.04505 & 1.48683 \\
\hline C & 0.18418 & 0.2957 & 0.06537 \\
\hline C & 0.86179 & -0.29341 & -1.03645 \\
\hline C & 1.77068 & -1.49952 & -1.03471 \\
\hline $\mathrm{O}$ & -0.33869 & 0.22257 & 2.37205 \\
\hline C & 1.26964 & -2.93115 & -0.81278 \\
\hline $\mathrm{H}$ & 2.48991 & -1.45361 & -1.84949 \\
\hline $\mathrm{H}$ & 3.29594 & -2.66689 & 0.00215 \\
\hline C & 1.77902 & -3.99086 & -1.77791 \\
\hline C & -0.15336 & -3.18474 & -0.34952 \\
\hline C & 2.19262 & -0.14578 & 3.35411 \\
\hline $\mathrm{H}$ & 1.05068 & -2.4643 & 1.94973 \\
\hline $\mathrm{H}$ & 2.77735 & -2.40609 & 2.21782 \\
\hline $\mathrm{H}$ & 2.64296 & 0.02528 & 1.25068 \\
\hline $\mathrm{H}$ & 1.75166 & -4.98694 & -1.31602 \\
\hline $\mathrm{H}$ & 1.1617 & -4.03042 & -2.68511 \\
\hline $\mathrm{H}$ & 2.81301 & -3.79499 & -2.08458 \\
\hline $\mathrm{H}$ & -0.20881 & -4.1184 & 0.22619 \\
\hline $\mathrm{H}$ & -0.55312 & -2.37964 & 0.26955 \\
\hline $\mathrm{H}$ & -0.82171 & -3.28932 & -1.21338 \\
\hline $\mathrm{H}$ & 3.20349 & -0.47994 & 3.61498 \\
\hline $\mathrm{H}$ & 1.47861 & -0.6444 & 4.01588 \\
\hline $\mathrm{H}$ & 2.12505 & 0.92971 & 3.55134 \\
\hline$C$ & 2.05238 & 1.15084 & -1.98826 \\
\hline $\mathrm{H}$ & 2.52017 & 0.57817 & -2.78753 \\
\hline $\mathrm{H}$ & 1.16683 & 1.67873 & -2.30613 \\
\hline C & 0.96816 & 2.61681 & 0.29894 \\
\hline $\mathrm{H}$ & 0.70088 & 2.85455 & 1.32923 \\
\hline & & & \\
\hline
\end{tabular}

\begin{tabular}{|l|l|l|l|}
\hline atom & \multicolumn{1}{|c|}{$\mathrm{X}$} & \multicolumn{1}{|c|}{$\mathrm{Y}$} & \multicolumn{1}{|c|}{$\mathrm{Z}$} \\
\hline $\mathrm{C}$ & 2.8861 & 1.72005 & -1.01313 \\
\hline $\mathrm{C}$ & 2.32196 & 2.3811 & 0.09265 \\
\hline $\mathrm{H}$ & 2.9733 & 2.50958 & 0.95744 \\
\hline $\mathrm{H}$ & 0.22419 & -0.32228 & -1.92139 \\
\hline $\mathrm{C}$ & 4.3627 & 1.3919 & -1.0111 \\
\hline $\mathrm{H}$ & 4.90221 & 2.01034 & -1.7411 \\
\hline $\mathrm{H}$ & 4.82005 & 1.56679 & -0.03168 \\
\hline $\mathrm{H}$ & 4.53908 & 0.34627 & -1.28985 \\
\hline $\mathrm{C}$ & -0.06124 & 3.13446 & -0.70992 \\
\hline $\mathrm{H}$ & 0.4875 & 3.48434 & -1.5924 \\
\hline $\mathrm{C}$ & -1.18926 & 0.90594 & -0.19227 \\
\hline $\mathrm{H}$ & -1.55535 & 1.26568 & 0.77415 \\
\hline $\mathrm{C}$ & -1.10693 & 2.08178 & -1.16825 \\
\hline $\mathrm{H}$ & -2.09788 & 2.54104 & -1.26283 \\
\hline $\mathrm{H}$ & -0.86488 & 1.69265 & -2.16448 \\
\hline $\mathrm{C}$ & -0.75463 & 4.36597 & -0.09077 \\
\hline $\mathrm{H}$ & -0.02327 & 5.11362 & 0.23609 \\
\hline $\mathrm{H}$ & -1.42053 & 4.83957 & -0.8207 \\
\hline $\mathrm{H}$ & -1.35707 & 4.08047 & 0.78056 \\
\hline $\mathrm{O}$ & -2.10443 & -0.06401 & -0.73306 \\
\hline $\mathrm{Si}$ & -3.5886 & -0.46309 & -0.04669 \\
\hline $\mathrm{C}$ & -4.44968 & -1.51249 & -1.35249 \\
\hline $\mathrm{H}$ & -5.42634 & -1.86104 & -0.99428 \\
\hline $\mathrm{H}$ & -4.61435 & -0.94539 & -2.27599 \\
\hline $\mathrm{H}$ & -3.85294 & -2.39696 & -1.60448 \\
\hline $\mathrm{C}$ & -3.35522 & -1.43579 & 1.55252 \\
\hline $\mathrm{H}$ & -2.94657 & -2.43349 & 1.3519 \\
\hline $\mathrm{H}$ & -2.65609 & -0.92045 & 2.22072 \\
\hline $\mathrm{H}$ & -4.30763 & -1.56468 & 2.08227 \\
\hline-4.59418 & 1.09973 & 0.30601 \\
\hline-4.09764 & 1.75265 & 1.03395 \\
\hline $\mathrm{H}$ & -4.76359 & 1.68297 & -0.60704 \\
\hline $\mathrm{H}$ & -5.57606 & 0.83888 & 0.72101 \\
\hline
\end{tabular}


Table 5.1-5: Atomic coordinates of structure 19.

\begin{tabular}{|c|c|c|c|}
\hline \multicolumn{4}{|c|}{19} \\
\hline atom & $X$ & Y & Z \\
\hline C & 2.78892 & -1.49313 & 0.81023 \\
\hline $\mathrm{C}$ & 1.60167 & -2.35924 & 1.18733 \\
\hline C & 0.67786 & -1.64194 & 2.1959 \\
\hline $\mathrm{C}$ & 0.5248 & -0.10815 & 2.02847 \\
\hline C & 0.23032 & 0.61128 & 0.67532 \\
\hline C & 1.0761 & 0.00346 & -0.48828 \\
\hline C & 2.50979 & -0.2634 & -0.03877 \\
\hline $\mathrm{O}$ & 0.56305 & 0.54396 & 3.06232 \\
\hline C & 3.35962 & -1.39916 & -0.5949 \\
\hline $\mathrm{H}$ & 3.06334 & 0.6389 & 0.21746 \\
\hline $\mathrm{H}$ & 3.51141 & -1.36765 & 1.61731 \\
\hline C & 4.85491 & -1.12766 & -0.68805 \\
\hline C & 2.86737 & -2.30177 & -1.7164 \\
\hline C & -0.69609 & -2.33416 & 2.29401 \\
\hline $\mathrm{H}$ & 1.02885 & -2.65368 & 0.30354 \\
\hline $\mathrm{H}$ & 1.94047 & -3.29803 & 1.64564 \\
\hline $\mathrm{H}$ & 1.14531 & -1.70962 & 3.18523 \\
\hline $\mathrm{H}$ & 5.42907 & -2.06262 & -0.64027 \\
\hline $\mathrm{H}$ & 5.1074 & -0.63128 & -1.63442 \\
\hline $\mathrm{H}$ & 5.1989 & -0.48282 & 0.1285 \\
\hline $\mathrm{H}$ & 3.36158 & -3.28075 & -1.66399 \\
\hline $\mathrm{H}$ & 1.789 & -2.47671 & -1.69938 \\
\hline $\mathrm{H}$ & 3.11172 & -1.86523 & -2.69344 \\
\hline $\mathrm{H}$ & -0.55804 & -3.39032 & 2.55382 \\
\hline $\mathrm{H}$ & -1.23154 & -2.28879 & 1.34083 \\
\hline $\mathrm{H}$ & -1.31862 & -1.87609 & 3.07032 \\
\hline C & 1.05686 & 0.87786 & -1.75744 \\
\hline $\mathrm{H}$ & 1.74786 & 0.44051 & -2.49043 \\
\hline $\mathrm{H}$ & 0.06196 & 0.83685 & -2.22334 \\
\hline$C$ & 0.45551 & 2.16273 & 0.85634 \\
\hline $\mathrm{H}$ & 0.99924 & 2.34103 & 1.78816 \\
\hline
\end{tabular}

\begin{tabular}{|c|c|c|c|}
\hline atom & $X$ & $Y$ & Z \\
\hline C & 1.44493 & 2.30964 & -1.4779 \\
\hline$C$ & 1.19733 & 2.83552 & -0.27354 \\
\hline $\mathrm{H}$ & 1.48279 & 3.87021 & -0.07551 \\
\hline $\mathrm{H}$ & 0.60051 & -0.93895 & -0.75739 \\
\hline C & 2.10613 & 3.08266 & -2.58816 \\
\hline $\mathrm{H}$ & 1.46779 & 3.11427 & -3.48286 \\
\hline $\mathrm{H}$ & 2.32464 & 4.11437 & -2.29278 \\
\hline $\mathrm{H}$ & 3.04788 & 2.60761 & -2.89738 \\
\hline C & -0.96917 & 2.81028 & 0.96924 \\
\hline $\mathrm{H}$ & -0.94088 & 3.81923 & 0.53978 \\
\hline C & -1.33984 & 0.47361 & 0.42037 \\
\hline $\mathrm{H}$ & -1.81393 & 0.16839 & 1.36277 \\
\hline C & -1.82042 & 1.89187 & 0.08171 \\
\hline $\mathrm{H}$ & -2.89836 & 2.01413 & 0.24519 \\
\hline $\mathrm{H}$ & -1.61583 & 2.0937 & -0.97608 \\
\hline C & -1.49709 & 2.93816 & 2.40571 \\
\hline $\mathrm{H}$ & -0.84802 & 3.59481 & 2.99656 \\
\hline $\mathrm{H}$ & -2.50392 & 3.37451 & 2.40482 \\
\hline $\mathrm{H}$ & -1.53756 & 1.97765 & 2.9262 \\
\hline $\mathrm{O}$ & -1.64076 & -0.50523 & -0.56214 \\
\hline Si & -3.16638 & -1.03508 & -1.04849 \\
\hline $\mathrm{C}$ & -2.84966 & -2.71068 & -1.84543 \\
\hline $\mathrm{H}$ & -3.77569 & -3.13346 & -2.25392 \\
\hline $\mathrm{H}$ & -2.13222 & -2.6241 & -2.66989 \\
\hline $\mathrm{H}$ & -2.44382 & -3.4282 & -1.12307 \\
\hline C & -4.3187 & -1.19667 & 0.43946 \\
\hline $\mathrm{H}$ & -3.91967 & -1.89014 & 1.18887 \\
\hline $\mathrm{H}$ & -4.49396 & -0.23401 & 0.93417 \\
\hline $\mathrm{H}$ & -5.29549 & -1.58096 & 0.12002 \\
\hline C & -3.9142 & 0.14402 & -2.31933 \\
\hline $\mathrm{H}$ & -4.11226 & 1.13574 & -1.89772 \\
\hline $\mathrm{H}$ & -3.24497 & 0.27379 & -3.17816 \\
\hline $\mathrm{H}$ & -4.86566 & -0.25174 & -2.6971 \\
\hline
\end{tabular}


Table 5.1-6: Atomic coordinates of structure 17b.

\begin{tabular}{|c|c|c|c|}
\hline \multicolumn{4}{|c|}{$17 b$} \\
\hline atom & $x$ & Y & $Z$ \\
\hline C & -1.62754 & 2.80983 & -0.04362 \\
\hline C & -1.46595 & 2.52542 & 1.43959 \\
\hline C & -0.19465 & 1.82374 & 1.97145 \\
\hline C & -0.04858 & 0.38411 & 1.47091 \\
\hline C & 0.23368 & 0.08647 & 0.03903 \\
\hline$C$ & -0.20712 & 0.78518 & -1.03048 \\
\hline C & -1.09476 & 1.95391 & -1.17163 \\
\hline 0 & -0.08525 & -0.54507 & 2.2783 \\
\hline C & -0.62177 & 3.41739 & -1.00034 \\
\hline $\mathrm{H}$ & -1.76926 & 1.82408 & -2.01825 \\
\hline $\mathrm{H}$ & -2.64103 & 3.16253 & -0.23773 \\
\hline C & -1.17872 & 4.41724 & -2.00433 \\
\hline C & 0.82143 & 3.71623 & -0.62317 \\
\hline C & -0.18108 & 1.86495 & 3.5055 \\
\hline $\mathrm{H}$ & -1.54982 & 3.48163 & 1.9738 \\
\hline $\mathrm{H}$ & -2.33245 & 1.92925 & 1.75856 \\
\hline $\mathrm{H}$ & 0.6812 & 2.37192 & 1.60867 \\
\hline $\mathrm{H}$ & -1.15459 & 5.43441 & -1.59124 \\
\hline $\mathrm{H}$ & -0.58539 & 4.42057 & -2.92751 \\
\hline $\mathrm{H}$ & -2.21697 & 4.19098 & -2.27101 \\
\hline $\mathrm{H}$ & 0.88175 & 4.53501 & 0.10516 \\
\hline $\mathrm{H}$ & 1.34468 & 2.84976 & -0.21362 \\
\hline $\mathrm{H}$ & 1.37758 & 4.03146 & -1.51452 \\
\hline $\mathrm{H}$ & 0.71166 & 1.37752 & 3.90714 \\
\hline $\mathrm{H}$ & -1.0521 & 1.34969 & 3.92247 \\
\hline $\mathrm{H}$ & -0.19433 & 2.90415 & 3.85204 \\
\hline$C$ & -4.77019 & -1.20012 & 0.79659 \\
\hline $\mathrm{H}$ & -5.67614 & -0.62055 & 0.95473 \\
\hline $\mathrm{H}$ & -4.16783 & -1.41512 & 1.67486 \\
\hline C & -2.02509 & -2.27631 & -0.0228 \\
\hline $\mathrm{H}$ & -1.95162 & -1.55557 & 0.78898 \\
\hline & & & \\
\hline
\end{tabular}

\begin{tabular}{|c|c|c|c|}
\hline atom & $X$ & $Y$ & Z \\
\hline $\mathrm{C}$ & -4.42364 & -1.63763 & -0.42492 \\
\hline C & -3.18447 & -2.39955 & -0.68663 \\
\hline $\mathrm{H}$ & -3.23165 & -3.09939 & -1.52457 \\
\hline $\mathrm{H}$ & 0.093 & 0.37622 & -1.99612 \\
\hline C & -5.3054 & -1.39377 & -1.62973 \\
\hline $\mathrm{H}$ & -6.21453 & -0.84668 & -1.36262 \\
\hline $\mathrm{H}$ & -4.77357 & -0.82236 & -2.40214 \\
\hline $\mathrm{H}$ & -5.60364 & -2.34367 & -2.09407 \\
\hline C & -0.78771 & -3.09408 & -0.30077 \\
\hline $\mathrm{H}$ & -1.06756 & -3.86975 & -1.02811 \\
\hline C & 1.07294 & -1.17752 & -0.21736 \\
\hline $\mathrm{H}$ & 1.391 & -1.56667 & 0.75489 \\
\hline C & 0.35759 & -2.3038 & -0.99071 \\
\hline $\mathrm{H}$ & 1.13926 & -3.02851 & -1.25305 \\
\hline $\mathrm{H}$ & -0.0134 & -1.89148 & -1.93736 \\
\hline C & -0.31121 & -3.80763 & 0.98134 \\
\hline $\mathrm{H}$ & -1.08436 & -4.49071 & 1.35041 \\
\hline $\mathrm{H}$ & 0.59478 & -4.39525 & 0.78664 \\
\hline $\mathrm{H}$ & -0.09251 & -3.08675 & 1.77496 \\
\hline $\mathrm{O}$ & 2.21856 & -0.81584 & -1.01006 \\
\hline Si & 3.7854 & -0.69833 & -0.40973 \\
\hline C & 4.81699 & -0.11272 & -1.86976 \\
\hline $\mathrm{H}$ & 5.8733 & -0.01432 & -1.59086 \\
\hline $\mathrm{H}$ & 4.75657 & -0.81954 & -2.70532 \\
\hline $\mathrm{H}$ & 4.47382 & 0.86337 & -2.23154 \\
\hline C & 3.87172 & 0.54364 & 1.01226 \\
\hline $\mathrm{H}$ & 3.5724 & 1.54638 & 0.68461 \\
\hline $\mathrm{H}$ & 3.22192 & 0.25419 & 1.84719 \\
\hline $\mathrm{H}$ & 4.89427 & 0.6135 & 1.40441 \\
\hline C & 4.3815 & -2.38582 & 0.19511 \\
\hline $\mathrm{H}$ & 3.75175 & -2.77833 & 1.00271 \\
\hline $\mathrm{H}$ & 4.37772 & -3.12145 & -0.61793 \\
\hline $\mathrm{H}$ & 5.4062 & -2.32143 & 0.58242 \\
\hline
\end{tabular}


Table 5.1-7: Atomic coordinates of structure 17b‡.

\begin{tabular}{|c|c|c|c|}
\hline \multicolumn{4}{|c|}{$17 b^{\ddagger}$} \\
\hline atom & $x$ & Y & Z \\
\hline C & 2.28089 & -2.24782 & 0.41738 \\
\hline C & 1.34028 & -2.53412 & 1.58939 \\
\hline C & 0.08116 & -1.65311 & 1.78082 \\
\hline C & 0.52604 & -0.19059 & 1.67488 \\
\hline C & 0.34706 & 0.4183 & 0.29385 \\
\hline C & 1.10425 & -0.10077 & -0.77002 \\
\hline C & 2.2072 & -1.12048 & -0.61611 \\
\hline $\mathrm{O}$ & 0.96546 & 0.3943 & 2.65088 \\
\hline C & 2.01177 & -2.57498 & -1.0389 \\
\hline $\mathrm{H}$ & 3.1942 & -0.73873 & -0.86446 \\
\hline $\mathrm{H}$ & 3.31072 & -2.45821 & 0.70587 \\
\hline C & 3.13708 & -3.23587 & -1.82136 \\
\hline C & 0.63401 & -3.05533 & -1.46587 \\
\hline C & -0.58065 & -1.95375 & 3.13133 \\
\hline $\mathrm{H}$ & 1.01984 & -3.58506 & 1.55565 \\
\hline $\mathrm{H}$ & 1.93087 & -2.42487 & 2.50797 \\
\hline $\mathrm{H}$ & -0.63068 & -1.85067 & 0.97472 \\
\hline $\mathrm{H}$ & 3.11871 & -4.32679 & -1.69381 \\
\hline $\mathrm{H}$ & 3.04768 & -3.02818 & -2.89617 \\
\hline $\mathrm{H}$ & 4.12012 & -2.87942 & -1.49278 \\
\hline $\mathrm{H}$ & 0.49389 & -4.11321 & -1.20654 \\
\hline $\mathrm{H}$ & -0.1783 & -2.48189 & -1.01418 \\
\hline $\mathrm{H}$ & 0.52222 & -2.97112 & -2.55497 \\
\hline $\mathrm{H}$ & -1.51385 & -1.39112 & 3.25115 \\
\hline $\mathrm{H}$ & 0.08417 & -1.679 & 3.95567 \\
\hline $\mathrm{H}$ & -0.81713 & -3.02088 & 3.21379 \\
\hline C & 2.30824 & 1.64207 & -1.65648 \\
\hline $\mathrm{H}$ & 2.88499 & 1.18871 & -2.46103 \\
\hline $\mathrm{H}$ & 1.34492 & 2.01673 & -1.95968 \\
\hline C & 0.85681 & 2.54829 & 0.70226 \\
\hline $\mathrm{H}$ & 0.49552 & 2.61757 & 1.72679 \\
\hline & & & \\
\hline
\end{tabular}

\begin{tabular}{|c|c|c|c|}
\hline atom & $X$ & $Y$ & Z \\
\hline $\mathrm{C}$ & 2.97351 & 2.14127 & -0.54096 \\
\hline C & 2.2471 & 2.53778 & 0.59658 \\
\hline $\mathrm{H}$ & 2.80381 & 2.57542 & 1.53245 \\
\hline $\mathrm{H}$ & 0.60297 & -0.13346 & -1.73496 \\
\hline C & 4.48014 & 2.00983 & -0.43965 \\
\hline $\mathrm{H}$ & 4.86307 & 1.23631 & -1.11441 \\
\hline $\mathrm{H}$ & 4.97464 & 2.94999 & -0.72037 \\
\hline $\mathrm{H}$ & 4.80325 & 1.76737 & 0.57916 \\
\hline C & -0.12888 & 3.15554 & -0.307 \\
\hline $\mathrm{H}$ & 0.44006 & 3.62057 & -1.11917 \\
\hline C & -1.10017 & 0.86159 & 0.01482 \\
\hline $\mathrm{H}$ & -1.55359 & 1.14985 & 0.9744 \\
\hline C & -1.08046 & 2.08767 & -0.90192 \\
\hline $\mathrm{H}$ & -2.09397 & 2.48646 & -1.02774 \\
\hline $\mathrm{H}$ & -0.75201 & 1.76665 & -1.89732 \\
\hline C & -0.91662 & 4.28044 & 0.39324 \\
\hline $\mathrm{H}$ & -0.23953 & 5.02118 & 0.83278 \\
\hline $\mathrm{H}$ & -1.57105 & 4.79804 & -0.31787 \\
\hline $\mathrm{H}$ & -1.54426 & 3.88159 & 1.20047 \\
\hline $\mathrm{O}$ & -1.86175 & -0.21044 & -0.55144 \\
\hline Si & -3.5362 & -0.40064 & -0.53305 \\
\hline C & -3.81016 & -2.24747 & -0.77275 \\
\hline $\mathrm{H}$ & -4.8796 & -2.48294 & -0.83662 \\
\hline $\mathrm{H}$ & -3.33643 & -2.59818 & -1.69713 \\
\hline $\mathrm{H}$ & -3.3886 & -2.82516 & 0.05792 \\
\hline C & -4.25089 & 0.17719 & 1.11783 \\
\hline $\mathrm{H}$ & -3.78948 & -0.34702 & 1.96345 \\
\hline $\mathrm{H}$ & -4.11878 & 1.25371 & 1.27862 \\
\hline $\mathrm{H}$ & -5.32856 & -0.02626 & 1.15201 \\
\hline C & -4.3468 & 0.54642 & -1.95249 \\
\hline $\mathrm{H}$ & -4.24423 & 1.63215 & -1.84259 \\
\hline $\mathrm{H}$ & -3.90448 & 0.26512 & -2.91554 \\
\hline $\mathrm{H}$ & -5.41972 & 0.32027 & -2.00149 \\
\hline
\end{tabular}


Table 5.1-8: Atomic coordinates of structure epi-19.

\begin{tabular}{|c|c|c|c|}
\hline \multicolumn{4}{|c|}{ epi-19 } \\
\hline atom & $x$ & Y & Z \\
\hline C & 2.96678 & -1.2874 & 0.63265 \\
\hline C & 2.04332 & -2.28626 & 1.33934 \\
\hline C & 0.54963 & -1.87893 & 1.48613 \\
\hline C & 0.50943 & -0.38055 & 1.81034 \\
\hline C & 0.20051 & 0.58601 & 0.6507 \\
\hline C & 1.02851 & 0.14572 & -0.59788 \\
\hline C & 2.49012 & -0.14289 & -0.25706 \\
\hline $\mathrm{O}$ & 0.7117 & 0.0001 & 2.95257 \\
\hline C & 3.27239 & -1.29957 & -0.85748 \\
\hline $\mathrm{H}$ & 3.06945 & 0.77123 & -0.13136 \\
\hline $\mathrm{H}$ & 3.82253 & -1.01779 & 1.24975 \\
\hline C & 4.70966 & -1.024 & -1.27445 \\
\hline C & 2.58828 & -2.3251 & -1.7469 \\
\hline C & -0.13274 & -2.71372 & 2.57754 \\
\hline $\mathrm{H}$ & 2.08517 & -3.27435 & 0.86268 \\
\hline $\mathrm{H}$ & 2.43269 & -2.42183 & 2.35506 \\
\hline $\mathrm{H}$ & 0.03482 & -2.0496 & 0.53549 \\
\hline $\mathrm{H}$ & 5.31463 & -1.94009 & -1.24093 \\
\hline $\mathrm{H}$ & 4.75636 & -0.634 & -2.30013 \\
\hline $\mathrm{H}$ & 5.18365 & -0.28678 & -0.61662 \\
\hline $\mathrm{H}$ & 3.16702 & -3.25796 & -1.76315 \\
\hline $\mathrm{H}$ & 1.57639 & -2.57836 & -1.41857 \\
\hline $\mathrm{H}$ & 2.51967 & -1.96472 & -2.78143 \\
\hline $\mathrm{H}$ & -1.21051 & -2.51806 & 2.61872 \\
\hline $\mathrm{H}$ & 0.2913 & -2.47739 & 3.55795 \\
\hline $\mathrm{H}$ & 0.00782 & -3.78361 & 2.38509 \\
\hline C & 0.96712 & 1.19271 & -1.72832 \\
\hline $\mathrm{H}$ & 1.66245 & 0.89021 & -2.5235 \\
\hline $\mathrm{H}$ & -0.03273 & 1.18432 & -2.18374 \\
\hline C & 0.438 & 2.07406 & 1.07759 \\
\hline $\mathrm{H}$ & 1.04892 & 2.09575 & 1.98662 \\
\hline & & & \\
\hline
\end{tabular}

\begin{tabular}{|c|c|c|c|}
\hline atom & $\mathrm{X}$ & $\mathrm{Y}$ & $\mathrm{Z}$ \\
\hline $\mathrm{C}$ & 1.31017 & 2.58549 & -1.2531 \\
\hline $\mathrm{C}$ & 1.09618 & 2.93012 & 0.02197 \\
\hline $\mathrm{H}$ & 1.35152 & 3.93976 & 0.34846 \\
\hline $\mathrm{H}$ & 0.55956 & -0.75782 & -0.98729 \\
\hline $\mathrm{C}$ & 1.88881 & 3.53681 & -2.26755 \\
\hline $\mathrm{H}$ & 1.21018 & 3.6627 & -3.12339 \\
\hline $\mathrm{H}$ & 2.07752 & 4.52624 & -1.83782 \\
\hline $\mathrm{H}$ & 2.83445 & 3.15449 & -2.67707 \\
\hline $\mathrm{C}$ & -0.97783 & 2.66695 & 1.3962 \\
\hline $\mathrm{H}$ & -0.98074 & 3.74076 & 1.17167 \\
\hline $\mathrm{C}$ & -1.37496 & 0.47343 & 0.41709 \\
\hline $\mathrm{H}$ & -1.81295 & -0.03706 & 1.28725 \\
\hline $\mathrm{C}$ & -1.87992 & 1.92358 & 0.39934 \\
\hline $\mathrm{H}$ & -2.94622 & 1.99587 & 0.64512 \\
\hline $\mathrm{H}$ & -1.73952 & 2.32724 & -0.61025 \\
\hline $\mathrm{C}$ & -1.41357 & 2.50102 & 2.85967 \\
\hline $\mathrm{H}$ & -0.73351 & 3.0423 & 3.52759 \\
\hline $\mathrm{H}$ & -2.42226 & 2.90684 & 3.00708 \\
\hline $\mathrm{H}$ & -1.41343 & 1.45623 & 3.18294 \\
\hline $\mathrm{O}$ & -1.70137 & -0.2715 & -0.74508 \\
\hline $\mathrm{Si}$ & -3.18578 & -0.9815 & -1.11409 \\
\hline $\mathrm{C}$ & -2.78495 & -2.23437 & -2.45932 \\
\hline $\mathrm{H}$ & -3.69369 & -2.73637 & -2.81327 \\
\hline $\mathrm{H}$ & -2.31532 & -1.74888 & -3.32274 \\
\hline $\mathrm{H}$ & -2.09668 & -3.00547 & -2.09444 \\
\hline $\mathrm{C}$ & -3.90584 & -1.83378 & 0.41005 \\
\hline $\mathrm{H}$ & -3.21544 & -2.58122 & 0.81878 \\
\hline $\mathrm{H}$ & -4.14637 & -1.12523 & 1.21144 \\
\hline $\mathrm{H}$ & -4.8359 & -2.35279 & 0.14628 \\
\hline $\mathrm{H}$ & -4.40535 & 0.30174 & -1.76959 \\
\hline-4.66591 & 1.04903 & -1.0114 \\
\hline $\mathrm{H}$ & -3.99232 & 0.83391 & -2.63461 \\
\hline $\mathrm{H}$ & & -0.18173 & -2.09064 \\
\hline
\end{tabular}




\subsection{DFT Calculations for epimerization with DBU}

The ground state electronic structures of the full models of compounds 17b, 17c, 17b-enolate, 17c-enolate, enol and the corresponding transition structures 17b-TS-enolate and 17c-TSenolate were calculated by density functional theory (DFT) methods using the Gaussian 16 program packages.[5] Open shell systems were calculated by the unrestricted Kohn-Sham approach (UKS). Geometry optimization followed by vibrational analysis was performed in solvent media. Solvent effects were described by the polarizable continuum model (PCM) with standard parameters for o-xylene..$^{[6]}$ The $6-31 \mathrm{G}(\mathrm{d})$ polarized double- $\zeta$ basis sets ${ }^{[7]}$ were employed together with the Becke Three-Parameter Hybrid Funtionals (B3LYP) ${ }^{[8]}$. To reduce computational complexity trimethylsilyl substituents were used instead of triethylsilyl substituents. Atomic coordinates of the calculated structures are provided in Tables 5.2-3-9.

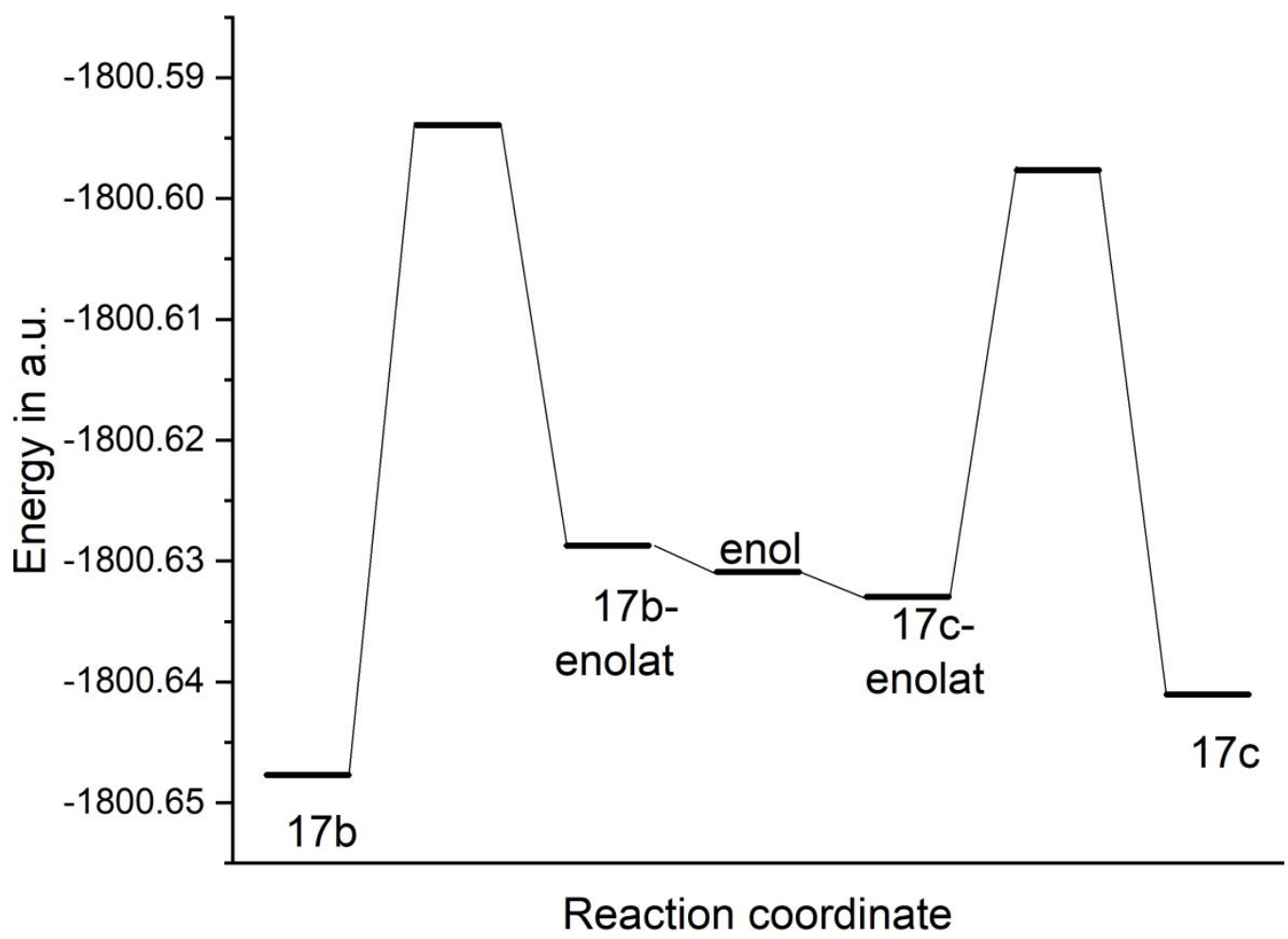

Figure 5.2-1: Comparison of Gibbs free energies of epimerization between 17b and 17c 
Table 5.2-1: Gibbs free energies $(G)$ of compounds $17 \mathrm{~b} / \mathrm{c}, 17 \mathrm{~b} / \mathrm{c}$-enolate and 17b/c-TS-enolate.

\begin{tabular}{|c|c|}
\hline compound & Gibbs free energy (G) [hartree] \\
\hline 17b & -1800.647680 \\
\hline 17c & -1800.641060 \\
\hline 17b-enolate & -1800.628730 \\
\hline 17c-enolate & -1800.632960 \\
\hline 17b-TS-enolate & -1800.59391 \\
\hline 17c-TS-enolate & -1800.59763 \\
\hline enol & -1800.63089 \\
\hline
\end{tabular}

Table 5.1-2: Gibbs free energy differences $(\Delta G)$.

\begin{tabular}{|c|c|c|}
\hline compounds & $\begin{array}{c}\text { Gibbs free energy } \\
\text { difference }(\Delta G) \text { [hartree] }\end{array}$ & $\begin{array}{c}\text { Gibbs free energy } \\
\text { difference }(\Delta G)[\mathrm{kcal} / \mathrm{mol}]\end{array}$ \\
\hline 17c-17c-TS-enolate & 0.043430 & $2.72527 \mathrm{E}+01$ \\
\hline 17c-TS-enolat - 17c-enolate & 0.035330 & $2.21699 \mathrm{E}+01$ \\
\hline 17c-17c-enolat & 0.008100 & $5.08283 \mathrm{E}+00$ \\
\hline $17 b-17 b-T S-e n o l a t e$ & 0.053770 & $3.37412 \mathrm{E}+01$ \\
\hline 17b-TS-enolat - 17b-enolate & 0.034820 & $2.18499 \mathrm{E}+01$ \\
\hline $17 b-17 b$-enolate & 0.018950 & 1.18913E+01 \\
\hline $17 c-17 b$ & 0.006620 & $4.15411 \mathrm{E}+00$ \\
\hline $\begin{array}{l}\text { (17c-TS-enolate) - } \\
\text { (17b-TS-enolate) }\end{array}$ & 0.00372 & $2.33434 \mathrm{E}+00$ \\
\hline 17c-enol & 0.010170 & $6.38177 \mathrm{E}+00$ \\
\hline 17b-enol & 0.016790 & $1.05359 \mathrm{E}+01$ \\
\hline (17c-enolate)-enol & 0.002070 & 1.29894E+00 \\
\hline (17b-enolate)-enol & 0.002160 & $1.35542 E+00$ \\
\hline
\end{tabular}


Table 5.2-3: Atomic coordinates of structure 17b

\begin{tabular}{|c|c|c|c|}
\hline \multicolumn{4}{|c|}{$17 b$} \\
\hline atom & $X$ & Y & Z \\
\hline$C$ & -0.22862 & 0.67903 & 1.795 \\
\hline$C$ & -0.70043 & 0.64079 & 0.35132 \\
\hline $\mathrm{C}$ & -0.01833 & -0.29404 & -0.67592 \\
\hline $\mathrm{C}$ & 1.44854 & 0.0543 & -0.93566 \\
\hline$C$ & 2.49023 & -0.13431 & 0.11395 \\
\hline $\mathrm{C}$ & 2.32968 & 0.02805 & 1.44585 \\
\hline $\mathrm{C}$ & 1.18994 & 0.46036 & 2.27461 \\
\hline 0 & 1.79772 & 0.42518 & -2.05751 \\
\hline $\mathrm{C}$ & 0.0491 & -0.4849 & 2.72335 \\
\hline $\mathrm{H}$ & 1.52386 & 1.12626 & 3.07085 \\
\hline $\mathrm{H}$ & -0.71445 & 1.50625 & 2.3139 \\
\hline $\mathrm{C}$ & -0.42335 & -0.31967 & 4.16103 \\
\hline $\mathrm{C}$ & 0.03729 & -1.93685 & 2.27125 \\
\hline $\mathrm{C}$ & -0.81904 & -0.28905 & -1.9855 \\
\hline $\mathrm{H}$ & -1.76712 & 0.38028 & 0.35177 \\
\hline $\mathrm{H}$ & -0.63573 & 1.66553 & -0.04203 \\
\hline $\mathrm{H}$ & -0.03101 & -1.31326 & -0.27451 \\
\hline $\mathrm{H}$ & -1.45369 & -0.68266 & 4.2734 \\
\hline $\mathrm{H}$ & 0.20751 & -0.89298 & 4.85248 \\
\hline $\mathrm{H}$ & -0.40424 & 0.72915 & 4.47732 \\
\hline $\mathrm{H}$ & -0.97851 & -2.26468 & 2.01494 \\
\hline $\mathrm{H}$ & 0.68569 & -2.12139 & 1.41242 \\
\hline $\mathrm{H}$ & 0.39344 & -2.5811 & 3.08478 \\
\hline $\mathrm{H}$ & -0.3795 & -0.96961 & -2.72096 \\
\hline $\mathrm{H}$ & -0.83203 & 0.71043 & -2.43266 \\
\hline $\mathrm{H}$ & -1.85082 & -0.59608 & -1.78727 \\
\hline C & 1.74151 & 5.02628 & -0.21386 \\
\hline $\mathrm{H}$ & 0.93134 & 5.68615 & 0.08587 \\
\hline $\mathrm{H}$ & 1.64512 & 4.5422 & -1.18172 \\
\hline C & 3.8124 & 2.79333 & -0.50974 \\
\hline $\mathrm{H}$ & 2.83714 & 2.49381 & -0.88778 \\
\hline C & 2.81182 & 4.83022 & 0.57317 \\
\hline C & 3.91721 & 3.91583 & 0.21765 \\
\hline $\mathrm{H}$ & 4.89623 & 4.18265 & 0.62327 \\
\hline $\mathrm{H}$ & 3.2341 & -0.12673 & 2.03599 \\
\hline $\mathrm{C}$ & 2.96844 & 5.56173 & 1.88805 \\
\hline $\mathrm{H}$ & 2.12988 & 6.23852 & 2.07782 \\
\hline
\end{tabular}

\begin{tabular}{|c|c|c|c|}
\hline $\mathrm{H}$ & 3.03995 & 4.85783 & 2.72793 \\
\hline $\mathrm{H}$ & 3.89342 & 6.15444 & 1.8978 \\
\hline $\mathrm{C}$ & 4.97186 & 1.90079 & -0.87843 \\
\hline $\mathrm{H}$ & 5.89067 & 2.41227 & -0.55696 \\
\hline $\mathrm{C}$ & 3.89098 & -0.4975 & -0.40821 \\
\hline $\mathrm{H}$ & 3.81321 & -0.64328 & -1.49006 \\
\hline $\mathrm{C}$ & 4.99573 & 0.54121 & -0.12785 \\
\hline $\mathrm{H}$ & 5.94217 & 0.04691 & -0.38315 \\
\hline $\mathrm{H}$ & 5.02665 & 0.73034 & 0.95249 \\
\hline $\mathrm{C}$ & 5.04961 & 1.72115 & -2.40874 \\
\hline $\mathrm{H}$ & 5.20558 & 2.68753 & -2.9011 \\
\hline $\mathrm{H}$ & 5.88404 & 1.06311 & -2.68232 \\
\hline $\mathrm{H}$ & 4.12431 & 1.28873 & -2.80124 \\
\hline $\mathrm{O}$ & 4.32004 & -1.71514 & 0.22993 \\
\hline $\mathrm{Si}$ & 4.37114 & -3.21433 & -0.52906 \\
\hline $\mathrm{C}$ & 4.92723 & -4.4125 & 0.81074 \\
\hline $\mathrm{H}$ & 5.00897 & -5.43319 & 0.41724 \\
\hline $\mathrm{H}$ & 5.90716 & -4.12952 & 1.21208 \\
\hline $\mathrm{H}$ & 4.21659 & -4.43116 & 1.64505 \\
\hline $\mathrm{C}$ & 2.66862 & -3.69573 & -1.19309 \\
\hline $\mathrm{H}$ & 1.93033 & -3.76561 & -0.38539 \\
\hline $\mathrm{H}$ & 2.29678 & -2.96974 & -1.92639 \\
\hline $\mathrm{H}$ & 2.70889 & -4.67212 & -1.69252 \\
\hline $\mathrm{C}$ & 5.61198 & -3.1845 & -1.95368 \\
\hline $\mathrm{H}$ & 5.34121 & -2.44546 & -2.71749 \\
\hline $\mathrm{H}$ & 6.61954 & -2.94073 & -1.59641 \\
\hline $\mathrm{H}$ & 5.66072 & -4.16255 & -2.44893 \\
\hline $\mathrm{C}$ & -5.23328 & 1.19792 & -0.75482 \\
\hline $\mathrm{C}$ & -5.35182 & -0.2513 & -0.29766 \\
\hline $\mathrm{C}$ & -6.09345 & 1.59867 & -1.9684 \\
\hline $\mathrm{C}$ & -7.88201 & -0.26569 & -0.54703 \\
\hline $\mathrm{C}$ & -7.50448 & 2.06875 & -1.58155 \\
\hline $\mathrm{C}$ & -8.11743 & 1.2456 & -0.43722 \\
\hline $\mathrm{H}$ & -5.45124 & 1.86583 & 0.09041 \\
\hline $\mathrm{H}$ & -8.01994 & -0.59378 & -1.59173 \\
\hline-6.1531 & 0.74962 & -2.66175 \\
\hline $\mathrm{H}$ & -8.15782 & 2.02091 & -2.46252 \\
\hline $\mathrm{H}$ & -5.58396 & 2.39762 & -2.51988 \\
\hline $\mathrm{H}$ & & \\
\hline $\mathrm{H}$ & & & \\
\hline $\mathrm{H}$ & & & \\
\hline & & & \\
\hline & & &
\end{tabular}




\begin{tabular}{|c|c|c|c|}
$H$ & -8.66068 & -0.77187 & 0.03324 \\
\hline$H$ & -7.47377 & 3.12345 & -1.27838 \\
\hline$H$ & -9.20082 & 1.41602 & -0.41435 \\
\hline$H$ & -7.73701 & 1.5834 & 0.53402 \\
\hline $\mathrm{C}$ & -6.69053 & -2.18942 & 0.37607 \\
\hline$H$ & -7.58753 & -2.32009 & 0.99313 \\
\hline$H$ & -6.82108 & -2.82338 & -0.51714 \\
\hline $\mathrm{C}$ & -4.21544 & -2.25423 & 0.31479 \\
\hline$H$ & -3.29935 & -2.40789 & 0.89981 \\
\hline$H$ & -4.13584 & -2.92657 & -0.5539 \\
\hline
\end{tabular}

\begin{tabular}{|c|c|c|c|}
$\mathrm{C}$ & -5.44605 & -2.61657 & 1.14331 \\
\hline $\mathrm{H}$ & -5.48748 & -3.69123 & 1.35542 \\
\hline $\mathrm{H}$ & -5.41315 & -2.08998 & 2.10527 \\
\hline $\mathrm{N}$ & -4.22662 & -0.87222 & -0.15237 \\
\hline $\mathrm{N}$ & -6.60988 & -0.77413 & -0.00658 \\
\hline
\end{tabular}

Table 5.2-4: Atomic coordinates of structure 17b-TS-enolate

\begin{tabular}{|c|c|c|c|}
\hline \multicolumn{4}{|c|}{ 17b-TS-enolate } \\
\hline atom & $x$ & $\mathrm{Y}$ & Z \\
\hline $\mathrm{C}$ & -0.09598 & 1.95494 & 2.16452 \\
\hline $\mathrm{C}$ & -0.40194 & 2.2244 & 0.69798 \\
\hline $\mathrm{C}$ & -0.47913 & 1.13311 & -0.36861 \\
\hline $\mathrm{C}$ & 0.56543 & 0.16825 & -0.5966 \\
\hline $\mathrm{C}$ & 1.66783 & -0.15594 & 0.40029 \\
\hline $\mathrm{C}$ & 1.82634 & 0.22739 & 1.68718 \\
\hline $\mathrm{C}$ & 1.04579 & 1.07101 & 2.611 \\
\hline $\mathrm{O}$ & 0.59718 & -0.50179 & -1.67411 \\
\hline $\mathrm{C}$ & -0.4061 & 0.76279 & 3.04458 \\
\hline $\mathrm{H}$ & 1.66468 & 1.46243 & 3.4179 \\
\hline $\mathrm{H}$ & -0.13757 & 2.8929 & 2.72191 \\
\hline $\mathrm{C}$ & -0.73589 & 1.08272 & 4.49666 \\
\hline $\mathrm{C}$ & -1.08062 & -0.50634 & 2.56551 \\
\hline $\mathrm{C}$ & -1.03455 & 1.72993 & -1.66639 \\
\hline $\mathrm{H}$ & -1.36266 & 2.76548 & 0.67389 \\
\hline $\mathrm{H}$ & 0.33595 & 2.99084 & 0.3899 \\
\hline $\mathrm{H}$ & -1.74398 & 0.08729 & -0.21423 \\
\hline $\mathrm{H}$ & -1.81409 & 1.25223 & 4.6255 \\
\hline $\mathrm{H}$ & -0.45118 & 0.25339 & 5.15761 \\
\hline $\mathrm{H}$ & -0.21534 & 1.98223 & 4.84417 \\
\hline $\mathrm{H}$ & -2.16529 & -0.36747 & 2.47441 \\
\hline $\mathrm{H}$ & -0.6983 & -0.84195 & 1.60254 \\
\hline $\mathrm{H}$ & -0.91313 & -1.31185 & 3.2934 \\
\hline $\mathrm{H}$ & -1.03659 & 0.98621 & -2.46522 \\
\hline $\mathrm{H}$ & -0.42539 & 2.5831 & -2.01429 \\
\hline $\mathrm{H}$ & -2.0574 & 2.11764 & -1.54546 \\
\hline
\end{tabular}

\begin{tabular}{|c|c|c|c|}
\hline $\mathrm{C}$ & 2.32554 & 4.8537 & -1.30746 \\
\hline $\mathrm{H}$ & 1.85341 & 5.80541 & -1.07658 \\
\hline $\mathrm{H}$ & 1.82287 & 4.22106 & -2.03361 \\
\hline $\mathrm{C}$ & 3.51236 & 2.02257 & -1.23783 \\
\hline $\mathrm{H}$ & 2.42428 & 1.99469 & -1.24087 \\
\hline $\mathrm{C}$ & 3.47957 & 4.48496 & -0.72707 \\
\hline $\mathrm{C}$ & 4.1331 & 3.18618 & -0.98982 \\
\hline $\mathrm{H}$ & 5.22489 & 3.19122 & -0.93809 \\
\hline $\mathrm{H}$ & 2.71857 & -0.17888 & 2.16511 \\
\hline $\mathrm{C}$ & 4.20963 & 5.40165 & 0.22961 \\
\hline $\mathrm{H}$ & 3.68886 & 6.356 & 0.35431 \\
\hline $\mathrm{H}$ & 4.31829 & 4.93625 & 1.21816 \\
\hline $\mathrm{H}$ & 5.22617 & 5.61245 & -0.13008 \\
\hline $\mathrm{C}$ & 4.22408 & 0.72621 & -1.53761 \\
\hline $\mathrm{H}$ & 5.29634 & 0.96088 & -1.60944 \\
\hline $\mathrm{C}$ & 2.77948 & -1.05413 & -0.16316 \\
\hline $\mathrm{H}$ & 2.40757 & -1.4627 & -1.10361 \\
\hline $\mathrm{C}$ & 4.12376 & -0.33793 & -0.40941 \\
\hline $\mathrm{H}$ & 4.84922 & -1.12651 & -0.65146 \\
\hline $\mathrm{H}$ & 4.45435 & 0.11154 & 0.53617 \\
\hline $\mathrm{C}$ & 3.77557 & 0.16748 & -2.90439 \\
\hline $\mathrm{H}$ & 4.00443 & 0.88004 & -3.70525 \\
\hline $\mathrm{H}$ & 4.29754 & -0.77194 & -3.12835 \\
\hline $\mathrm{H}$ & 2.69848 & -0.02825 & -2.9142 \\
\hline $\mathrm{O}$ & 3.07172 & -2.12934 & 0.76419 \\
\hline $\mathrm{Si}$ & 2.8427 & -3.74523 & 0.39279 \\
\hline $\mathrm{C}$ & 3.2943 & -4.68363 & 1.96273 \\
\hline $\mathrm{H}$ & 3.17185 & -5.76538 & 1.82603 \\
\hline & & & \\
\hline
\end{tabular}




\begin{tabular}{|c|c|c|c|}
$\mathrm{H}$ & 4.33651 & -4.4995 & 2.24858 \\
\hline $\mathrm{H}$ & 2.65895 & -4.37755 & 2.80198 \\
\hline $\mathrm{C}$ & 1.04389 & -4.06603 & -0.09066 \\
\hline $\mathrm{H}$ & 0.37676 & -3.91075 & 0.76608 \\
\hline $\mathrm{H}$ & 0.73519 & -3.37659 & -0.88541 \\
\hline $\mathrm{H}$ & 0.89767 & -5.09171 & -0.4521 \\
\hline $\mathrm{C}$ & 3.96991 & -4.2772 & -1.03039 \\
\hline $\mathrm{H}$ & 3.76532 & -3.70637 & -1.94433 \\
\hline $\mathrm{H}$ & 5.02638 & -4.13024 & -0.7757 \\
\hline $\mathrm{H}$ & 3.8284 & -5.33933 & -1.2682 \\
\hline $\mathrm{C}$ & -4.09466 & 1.06316 & 0.36741 \\
\hline $\mathrm{C}$ & -3.87184 & -0.23567 & -0.38761 \\
\hline $\mathrm{C}$ & -5.05769 & 2.06826 & -0.28782 \\
\hline $\mathrm{C}$ & -6.29738 & -0.48449 & -1.06667 \\
\hline $\mathrm{C}$ & -6.52714 & 1.81257 & 0.07795 \\
\hline $\mathrm{C}$ & -6.87805 & 0.31738 & 0.10165 \\
\hline $\mathrm{H}$ & -4.43607 & 0.8167 & 1.38166 \\
\hline $\mathrm{H}$ & -6.40911 & 0.073 & -2.00842 \\
\hline $\mathrm{H}$ & -4.92333 & 2.04054 & -1.37642 \\
\hline $\mathrm{H}$ & -7.17497 & 2.33462 & -0.63707 \\
\hline $\mathrm{H}$ & -3.10785 & 1.51086 & 0.48869 \\
\hline & & & \\
\hline
\end{tabular}

\begin{tabular}{|c|c|c|c|}
$\mathrm{H}$ & -4.77142 & 3.07904 & 0.02207 \\
\hline $\mathrm{H}$ & -6.88309 & -1.40099 & -1.17804 \\
\hline $\mathrm{H}$ & -6.74469 & 2.24132 & 1.06419 \\
\hline $\mathrm{H}$ & -7.96756 & 0.20103 & 0.06723 \\
\hline $\mathrm{H}$ & -6.55624 & -0.14828 & 1.04072 \\
\hline $\mathrm{C}$ & -4.57752 & -2.14314 & -1.72696 \\
\hline $\mathrm{H}$ & -5.46103 & -2.78693 & -1.72012 \\
\hline $\mathrm{H}$ & -4.38806 & -1.85333 & -2.77007 \\
\hline $\mathrm{C}$ & -2.20207 & -1.91252 & -1.04189 \\
\hline $\mathrm{H}$ & -1.40525 & -2.32243 & -0.41644 \\
\hline $\mathrm{H}$ & -1.74973 & -1.70227 & -2.01638 \\
\hline $\mathrm{C}$ & -3.37541 & -2.87589 & -1.14386 \\
\hline $\mathrm{H}$ & -3.1166 & -3.72875 & -1.77894 \\
\hline $\mathrm{H}$ & -3.63456 & -3.26658 & -0.15277 \\
\hline $\mathrm{N}$ & -2.6256 & -0.64683 & -0.43906 \\
\hline $\mathrm{N}$ & -4.89514 & -0.94117 & -0.93066 \\
\hline
\end{tabular}

Table 5.2-5: Atomic coordinates of structure 17b-enolate

\begin{tabular}{|c|c|c|c|}
\hline \multicolumn{4}{|c|}{ 17b-enolate } \\
\hline atom & $\mathrm{X}$ & $\mathrm{Y}$ & $\mathrm{Z}$ \\
\hline $\mathrm{C}$ & 2.02717 & 2.5427 & 1.8594 \\
\hline $\mathrm{C}$ & 1.6218 & 2.74849 & 0.40534 \\
\hline $\mathrm{C}$ & 0.71003 & 1.79329 & -0.34509 \\
\hline $\mathrm{C}$ & 0.72928 & 0.43833 & -0.2691 \\
\hline $\mathrm{C}$ & 1.63319 & -0.41332 & 0.55071 \\
\hline $\mathrm{C}$ & 2.29245 & -0.06712 & 1.67847 \\
\hline $\mathrm{C}$ & 2.39845 & 1.19597 & 2.4354 \\
\hline $\mathrm{O}$ & -0.12861 & -0.33691 & -1.02655 \\
\hline $\mathrm{C}$ & 1.20567 & 2.00318 & 3.00656 \\
\hline $\mathrm{H}$ & 3.26612 & 1.19519 & 3.09377 \\
\hline $\mathrm{H}$ & 2.70385 & 3.3436 & 2.16344 \\
\hline $\mathrm{C}$ & 1.44872 & 2.65845 & 4.35976 \\
\hline $\mathrm{C}$ & -0.21736 & 1.49912 & 2.85538 \\
\hline $\mathrm{C}$ & -0.18741 & 2.50773 & -1.3321 \\
\hline $\mathrm{H}$ & 1.15633 & 3.7428 & 0.34594 \\
\hline
\end{tabular}

\begin{tabular}{|c|c|c|c|}
$\mathrm{H}$ & 2.56389 & 2.84774 & -0.16155 \\
\hline $\mathrm{H}$ & -1.04851 & 0.04624 & -1.12652 \\
\hline $\mathrm{H}$ & 0.78781 & 3.5252 & 4.49759 \\
\hline $\mathrm{H}$ & 1.24838 & 1.956 & 5.1793 \\
\hline $\mathrm{H}$ & 2.48189 & 3.00883 & 4.46318 \\
\hline $\mathrm{H}$ & -0.91657 & 2.34162 & 2.76141 \\
\hline $\mathrm{H}$ & -0.34744 & 0.85168 & 1.99001 \\
\hline $\mathrm{H}$ & -0.50934 & 0.93142 & 3.74866 \\
\hline $\mathrm{H}$ & -0.61823 & 1.83608 & -2.07624 \\
\hline $\mathrm{H}$ & 0.38421 & 3.27987 & -1.86601 \\
\hline $\mathrm{H}$ & -1.01865 & 3.0292 & -0.83349 \\
\hline $\mathrm{C}$ & 4.6867 & 2.89635 & -2.30143 \\
\hline $\mathrm{H}$ & 4.9451 & 3.95014 & -2.23331 \\
\hline $\mathrm{H}$ & 3.74474 & 2.65285 & -2.7848 \\
\hline $\mathrm{C}$ & 3.9446 & -0.02949 & -1.74673 \\
\hline $\mathrm{H}$ & 3.09408 & 0.62922 & -1.57777 \\
\hline $\mathrm{C}$ & 5.50253 & 1.94208 & -1.82306 \\
\hline
\end{tabular}




\begin{tabular}{|c|c|c|c|}
\hline $\mathrm{C}$ & 5.16974 & 0.50335 & -1.87214 \\
\hline $\mathrm{H}$ & 6.02128 & -0.17189 & -1.98796 \\
\hline $\mathrm{H}$ & 2.86437 & -0.87571 & 2.1311 \\
\hline $\mathrm{C}$ & 6.84699 & 2.28218 & -1.21914 \\
\hline $\mathrm{H}$ & 7.04179 & 3.35868 & -1.24264 \\
\hline $\mathrm{H}$ & 6.90984 & 1.94264 & -0.17692 \\
\hline $\mathrm{H}$ & 7.65762 & 1.77698 & -1.76193 \\
\hline $\mathrm{C}$ & 3.64657 & -1.50594 & -1.84133 \\
\hline $\mathrm{H}$ & 4.58595 & -2.0044 & -2.12091 \\
\hline $\mathrm{C}$ & 1.82677 & -1.86564 & 0.07351 \\
\hline $\mathrm{H}$ & 1.08405 & -2.07533 & -0.69569 \\
\hline $\mathrm{C}$ & 3.22861 & -2.16387 & -0.49727 \\
\hline $\mathrm{H}$ & 3.2676 & -3.2514 & -0.64508 \\
\hline $\mathrm{H}$ & 3.97647 & -1.9232 & 0.26872 \\
\hline $\mathrm{C}$ & 2.62638 & -1.78124 & -2.96637 \\
\hline $\mathrm{H}$ & 3.02159 & -1.44953 & -3.93314 \\
\hline $\mathrm{H}$ & 2.40858 & -2.85432 & -3.03911 \\
\hline $\mathrm{H}$ & 1.68093 & -1.25663 & -2.79058 \\
\hline $\mathrm{O}$ & 1.65196 & -2.78687 & 1.17013 \\
\hline $\mathrm{Si}$ & 0.24587 & -3.65757 & 1.4556 \\
\hline $\mathrm{C}$ & 0.60505 & -4.64431 & 3.0197 \\
\hline $\mathrm{H}$ & -0.25061 & -5.2732 & 3.29545 \\
\hline $\mathrm{H}$ & 1.47264 & -5.30048 & 2.88389 \\
\hline $\mathrm{H}$ & 0.81796 & -3.97984 & 3.86519 \\
\hline $\mathrm{C}$ & -1.24335 & -2.52395 & 1.70531 \\
\hline $\mathrm{H}$ & -1.10652 & -1.87642 & 2.57921 \\
\hline $\mathrm{H}$ & -1.39207 & -1.87995 & 0.83097 \\
\hline $\mathrm{H}$ & -2.15946 & -3.1092 & 1.85805 \\
\hline $\mathrm{C}$ & -0.10189 & -4.82766 & 0.01006 \\
\hline $\mathrm{H}$ & -0.2651 & -4.28119 & -0.92669 \\
\hline $\mathrm{H}$ & 0.73238 & -5.52133 & -0.149 \\
\hline $\mathrm{H}$ & -1.00197 & -5.42625 & 0.20031 \\
\hline & & & \\
\hline $\mathrm{H}$ & & \\
\hline $\mathrm{H}$ & & \\
\hline $\mathrm{H}$ & & & \\
\hline $\mathrm{H}$ & & & \\
\hline & & &
\end{tabular}

\begin{tabular}{|c|c|c|c|}
\hline $\mathrm{C}$ & -3.50607 & 1.02283 & 0.5186 \\
\hline $\mathrm{C}$ & -3.80577 & 0.47574 & -0.8706 \\
\hline $\mathrm{C}$ & -4.21517 & 2.33594 & 0.89931 \\
\hline $\mathrm{C}$ & -6.28753 & 0.98219 & -0.69505 \\
\hline $\mathrm{C}$ & -5.60359 & 2.11551 & 1.51911 \\
\hline $\mathrm{C}$ & -6.39011 & 0.98644 & 0.83468 \\
\hline $\mathrm{H}$ & -3.7283 & 0.24809 & 1.26497 \\
\hline $\mathrm{H}$ & -6.36433 & 2.01141 & -1.08286 \\
\hline $\mathrm{H}$ & -4.29285 & 2.97457 & 0.00979 \\
\hline $\mathrm{H}$ & -6.17681 & 3.05016 & 1.46736 \\
\hline $\mathrm{H}$ & -2.42484 & 1.16588 & 0.54084 \\
\hline $\mathrm{H}$ & -3.58455 & 2.88623 & 1.60733 \\
\hline $\mathrm{H}$ & -7.15388 & 0.44357 & -1.09142 \\
\hline $\mathrm{H}$ & -5.49968 & 1.87589 & 2.58516 \\
\hline $\mathrm{H}$ & -7.45092 & 1.07754 & 1.09791 \\
\hline $\mathrm{H}$ & -6.06947 & 0.0058 & 1.20552 \\
\hline $\mathrm{C}$ & -5.36723 & -0.21135 & -2.63095 \\
\hline $\mathrm{H}$ & -6.32693 & -0.74019 & -2.61418 \\
\hline $\mathrm{H}$ & -5.47236 & 0.62449 & -3.34125 \\
\hline $\mathrm{C}$ & -2.91653 & -0.42776 & -2.90503 \\
\hline $\mathrm{H}$ & -2.07726 & -1.11252 & -3.0723 \\
\hline $\mathrm{H}$ & -2.81717 & 0.37154 & -3.6553 \\
\hline $\mathrm{C}$ & -4.25068 & -1.14601 & -3.07739 \\
\hline $\mathrm{H}$ & -4.41131 & -1.44785 & -4.11827 \\
\hline $\mathrm{H}$ & -4.26488 & -2.05385 & -2.46213 \\
\hline $\mathrm{N}$ & -2.7626 & 0.14755 & -1.57031 \\
\hline $\mathrm{N}$ & -5.11546 & 0.30197 & -1.27628 \\
\hline & & & \\
\hline & & \\
\hline $\mathrm{H}$ & & \\
\hline $\mathrm{H}$ & & & \\
\hline & & &
\end{tabular}

Table 5.2-6: Atomic coordinates of structure enol

\begin{tabular}{|c|c|c|c|}
\hline \multicolumn{4}{|c|}{ enol } \\
\hline atom & $\mathrm{X}$ & $\mathrm{Y}$ & $\mathrm{Z}$ \\
\hline $\mathrm{C}$ & -3.65168 & -0.61052 & 2.33221 \\
\hline $\mathrm{C}$ & -2.39986 & -1.45585 & 2.10177 \\
\hline $\mathrm{C}$ & -1.06832 & -0.77896 & 1.84471 \\
\hline $\mathrm{C}$ & -0.8471 & 0.05336 & 0.79638 \\
\hline
\end{tabular}

\begin{tabular}{|c|c|c|c|}
$\mathrm{C}$ & -1.87934 & 0.60056 & -0.12014 \\
\hline $\mathrm{C}$ & -3.19554 & 0.77305 & 0.15192 \\
\hline $\mathrm{C}$ & -4.04515 & 0.44758 & 1.31731 \\
\hline $\mathrm{O}$ & 0.40341 & 0.57634 & 0.53402 \\
\hline $\mathrm{C}$ & -3.76579 & 0.82278 & 2.7935 \\
\hline $\mathrm{H}$ & -5.10301 & 0.4846 & 1.0612 \\
\hline
\end{tabular}




\begin{tabular}{|c|c|c|c|}
\hline $\mathrm{H}$ & -4.48202 & -1.23781 & 2.66326 \\
\hline C & -5.0099 & 1.13547 & 3.61714 \\
\hline$C$ & -2.59485 & 1.70941 & 3.18054 \\
\hline C & 0.01723 & -1.10542 & 2.84284 \\
\hline $\mathrm{H}$ & -2.27552 & -2.10442 & 2.97878 \\
\hline $\mathrm{H}$ & -2.64312 & -2.13605 & 1.27053 \\
\hline $\mathrm{H}$ & 1.14356 & -0.09594 & 0.62936 \\
\hline $\mathrm{H}$ & -4.81738 & 0.97392 & 4.68651 \\
\hline $\mathrm{H}$ & -5.3124 & 2.1829 & 3.48957 \\
\hline $\mathrm{H}$ & -5.85892 & 0.50368 & 3.33278 \\
\hline $\mathrm{H}$ & -2.21012 & 1.42776 & 4.16994 \\
\hline $\mathrm{H}$ & -1.76959 & 1.66889 & 2.47321 \\
\hline $\mathrm{H}$ & -2.93118 & 2.75239 & 3.24758 \\
\hline $\mathrm{H}$ & 0.89498 & -0.46576 & 2.73029 \\
\hline $\mathrm{H}$ & 0.35489 & -2.14964 & 2.76289 \\
\hline $\mathrm{H}$ & -0.36634 & -0.98441 & 3.86595 \\
\hline $\mathrm{C}$ & -2.1221 & -4.7574 & -0.17403 \\
\hline $\mathrm{H}$ & -2.48224 & -5.51454 & 0.51785 \\
\hline $\mathrm{H}$ & -1.13149 & -4.35326 & 0.01521 \\
\hline $\mathrm{C}$ & -1.67397 & -2.22654 & -1.85738 \\
\hline $\mathrm{H}$ & -1.35308 & -2.07094 & -0.82806 \\
\hline C & -2.86505 & -4.34926 & -1.21597 \\
\hline $\mathrm{C}$ & -2.42275 & -3.29934 & -2.15691 \\
\hline $\mathrm{H}$ & -2.78191 & -3.40936 & -3.18319 \\
\hline $\mathrm{H}$ & -3.76014 & 1.23938 & -0.65441 \\
\hline C & -4.21392 & -4.96597 & -1.51333 \\
\hline $\mathrm{H}$ & -4.46622 & -5.75392 & -0.7973 \\
\hline $\mathrm{H}$ & -5.00906 & -4.20938 & -1.48812 \\
\hline $\mathrm{H}$ & -4.22973 & -5.40295 & -2.52115 \\
\hline $\mathrm{C}$ & -1.22281 & -1.20048 & -2.86728 \\
\hline $\mathrm{H}$ & -1.57245 & -1.54563 & -3.85092 \\
\hline $\mathrm{C}$ & -1.39569 & 1.08047 & -1.50063 \\
\hline $\mathrm{H}$ & -0.30555 & 1.10154 & -1.4842 \\
\hline $\mathrm{C}$ & -1.85765 & 0.20539 & -2.68483 \\
\hline $\mathrm{H}$ & -1.62485 & 0.78053 & -3.59061 \\
\hline $\mathrm{H}$ & -2.94973 & 0.10617 & -2.64648 \\
\hline C & 0.31847 & -1.13769 & -2.92765 \\
\hline $\mathrm{H}$ & 0.73052 & -2.10825 & -3.22655 \\
\hline $\mathrm{H}$ & 0.64861 & -0.38925 & -3.65895 \\
\hline $\mathrm{H}$ & 0.75408 & -0.87223 & -1.95797 \\
\hline
\end{tabular}

\begin{tabular}{|c|c|c|c|}
\hline $\mathrm{O}$ & -1.89253 & 2.40409 & -1.78618 \\
\hline $\mathrm{Si}$ & -1.04094 & 3.82187 & -1.49626 \\
\hline $\mathrm{C}$ & -2.1577 & 5.18783 & -2.15764 \\
\hline $\mathrm{H}$ & -1.69436 & 6.1737 & -2.02676 \\
\hline $\mathrm{H}$ & -2.36147 & 5.05271 & -3.22623 \\
\hline $\mathrm{H}$ & -3.12019 & 5.19949 & -1.63298 \\
\hline $\mathrm{C}$ & -0.69539 & 4.07848 & 0.34097 \\
\hline $\mathrm{H}$ & -1.62689 & 4.13345 & 0.9161 \\
\hline $\mathrm{H}$ & -0.10159 & 3.25123 & 0.74585 \\
\hline $\mathrm{H}$ & -0.13986 & 5.01053 & 0.50798 \\
\hline $\mathrm{C}$ & 0.59937 & 3.80571 & -2.43982 \\
\hline $\mathrm{H}$ & 1.26 & 3.00095 & -2.09577 \\
\hline $\mathrm{H}$ & 0.44024 & 3.6724 & -3.51661 \\
\hline $\mathrm{H}$ & 1.13593 & 4.75252 & -2.29711 \\
\hline $\mathrm{C}$ & 3.80184 & 1.0047 & 0.57569 \\
\hline $\mathrm{C}$ & 3.78678 & -0.51296 & 0.43705 \\
\hline $\mathrm{C}$ & 4.8243 & 1.58192 & 1.57315 \\
\hline $\mathrm{C}$ & 6.32109 & -0.68313 & 0.44135 \\
\hline $\mathrm{C}$ & 6.201 & 1.84304 & 0.94349 \\
\hline $\mathrm{C}$ & 6.62437 & 0.74053 & -0.03961 \\
\hline $\mathrm{H}$ & 3.95542 & 1.45305 & -0.41545 \\
\hline $\mathrm{H}$ & 6.5695 & -0.78265 & 1.5108 \\
\hline $\mathrm{H}$ & 4.92024 & 0.89889 & 2.42718 \\
\hline $\mathrm{H}$ & 6.95205 & 1.93884 & 1.73824 \\
\hline $\mathrm{H}$ & 2.79147 & 1.2794 & 0.88065 \\
\hline $\mathrm{H}$ & 4.42858 & 2.51776 & 1.98398 \\
\hline $\mathrm{H}$ & 6.98766 & -1.36959 & -0.08989 \\
\hline $\mathrm{H}$ & 6.1897 & 2.80347 & 0.41228 \\
\hline $\mathrm{H}$ & 7.70482 & 0.81031 & -0.21474 \\
\hline $\mathrm{H}$ & 6.14954 & 0.88413 & -1.01731 \\
\hline $\mathrm{C}$ & 4.91603 & -2.65926 & 0.07344 \\
\hline $\mathrm{H}$ & 5.72891 & -2.9695 & -0.59288 \\
\hline $\mathrm{H}$ & 5.111 & -3.11965 & 1.0553 \\
\hline $\mathrm{C}$ & 2.46351 & -2.51041 & 0.37455 \\
\hline $\mathrm{H}$ & 1.48137 & -2.70705 & -0.07055 \\
\hline $\mathrm{H}$ & 2.44929 & -2.97177 & 1.37351 \\
\hline $\mathrm{H}$ & 3.57294 & -3.12615 & -0.47129 \\
\hline $\mathrm{H}$ & 3.46649 & -2.80173 & -1.51357 \\
\hline $\mathrm{H}$ & & -1.06405 & 0.51757 \\
\hline $\mathrm{H}$ & & \\
\hline $\mathrm{H}$ & & & \\
\hline $\mathrm{H}$ & & & \\
\hline & & &
\end{tabular}


\begin{tabular}{|l|l|l|l|}
$\mathrm{N}$ & 4.96167 & -1.19359 & 0.18311 \\
\hline
\end{tabular}

Table 5.2-7: Atomic coordinates of structure 17c-enolate

\begin{tabular}{|c|c|c|c|}
\hline \multicolumn{4}{|c|}{ 17c-enolate } \\
\hline atom & $X$ & $\mathrm{Y}$ & Z \\
\hline C & -0.85996 & -2.395 & -2.39521 \\
\hline C & -1.2544 & -3.01202 & -1.06129 \\
\hline C & -0.52406 & -2.39618 & 0.12217 \\
\hline C & -0.70264 & -1.0919 & 0.43997 \\
\hline C & -1.65491 & -0.17188 & -0.24004 \\
\hline C & -1.87665 & -0.14131 & -1.56845 \\
\hline C & -1.16968 & -0.93198 & -2.61448 \\
\hline $\mathrm{O}$ & -0.07164 & $-4.88 \mathrm{E}-01$ & 1.51007 \\
\hline $\mathrm{C}$ & -1.86528 & -1.99446 & -3.4644 \\
\hline $\mathrm{H}$ & -0.42126 & -0.34677 & -3.15485 \\
\hline $\mathrm{H}$ & 0.10511 & -2.73746 & -2.76928 \\
\hline$C$ & -1.40004 & -2.11644 & -4.90732 \\
\hline C & -3.35344 & -2.25439 & -3.297 \\
\hline C & 0.3608 & -3.31334 & 0.9272 \\
\hline $\mathrm{H}$ & -2.33538 & -2.90306 & -0.91295 \\
\hline $\mathrm{H}$ & -1.0595 & -4.09156 & -1.08775 \\
\hline $\mathrm{H}$ & 0.91958 & -0.66155 & 1.50726 \\
\hline $\mathrm{H}$ & -1.59151 & -3.12456 & -5.29915 \\
\hline $\mathrm{H}$ & -1.92799 & -1.40391 & -5.5553 \\
\hline $\mathrm{H}$ & -0.3256 & -1.92129 & -5.00303 \\
\hline $\mathrm{H}$ & -3.60036 & -3.29831 & -3.53175 \\
\hline $\mathrm{H}$ & -3.71125 & -2.03897 & -2.28726 \\
\hline $\mathrm{H}$ & -3.92651 & -1.61932 & -3.98545 \\
\hline $\mathrm{H}$ & 1.2465 & -3.63445 & 0.35858 \\
\hline $\mathrm{H}$ & -0.18393 & -4.23017 & 1.19415 \\
\hline $\mathrm{H}$ & 0.71158 & -2.84385 & 1.84871 \\
\hline $\mathrm{C}$ & -0.81073 & 4.82547 & -2.16886 \\
\hline $\mathrm{H}$ & -1.008 & 5.78603 & -2.6392 \\
\hline $\mathrm{H}$ & -1.67082 & 4.19207 & -1.97688 \\
\hline $\mathrm{C}$ & 0.16843 & 2.52553 & -0.20434 \\
\hline $\mathrm{H}$ & 0.59976 & 1.58335 & 0.12919 \\
\hline C & 0.44042 & 4.43872 & -1.87112 \\
\hline C & 0.77499 & 3.14894 & -1.2309 \\
\hline $\mathrm{H}$ & 1.67506 & 2.67465 & -1.62947 \\
\hline
\end{tabular}

\begin{tabular}{|c|c|c|c|}
\hline$H$ & -2.60611 & 0.57982 & -1.93845 \\
\hline $\mathrm{C}$ & 1.6306 & 5.30612 & -2.22329 \\
\hline $\mathrm{H}$ & 1.32613 & 6.22366 & -2.73614 \\
\hline $\mathrm{H}$ & 2.19742 & 5.58503 & -1.32527 \\
\hline $\mathrm{H}$ & 2.32807 & 4.76511 & -2.87753 \\
\hline $\mathrm{C}$ & -0.99813 & 3.00731 & 0.62381 \\
\hline $\mathrm{H}$ & -1.1867 & 4.05546 & 0.3607 \\
\hline $\mathrm{C}$ & -2.44691 & 0.77404 & 0.67637 \\
\hline $\mathrm{H}$ & -2.09992 & 0.62148 & 1.70207 \\
\hline $\mathrm{C}$ & -2.33788 & 2.27392 & 0.34066 \\
\hline $\mathrm{H}$ & -3.11265 & 2.77562 & 0.93535 \\
\hline $\mathrm{H}$ & -2.61522 & 2.41522 & -0.71082 \\
\hline $\mathrm{C}$ & -0.62523 & 2.95943 & 2.12076 \\
\hline $\mathrm{H}$ & 0.22331 & 3.62217 & 2.32674 \\
\hline $\mathrm{H}$ & -1.46737 & 3.28796 & 2.74291 \\
\hline $\mathrm{H}$ & -0.34421 & 1.94674 & 2.42661 \\
\hline $\mathrm{O}$ & -3.84994 & 0.4393 & 0.58308 \\
\hline $\mathrm{Si}$ & -4.73384 & -0.29868 & 1.80358 \\
\hline $\mathrm{C}$ & -6.47948 & -0.44461 & 1.11311 \\
\hline $\mathrm{H}$ & -7.15032 & -0.92706 & 1.83478 \\
\hline $\mathrm{H}$ & -6.89623 & 0.54112 & 0.87613 \\
\hline $\mathrm{H}$ & -6.49131 & -1.04203 & 0.19411 \\
\hline $\mathrm{C}$ & -4.0381 & -2.00565 & 2.21624 \\
\hline $\mathrm{H}$ & -4.09446 & -2.6753 & 1.34982 \\
\hline $\mathrm{H}$ & -2.98684 & -1.95167 & 2.52232 \\
\hline $\mathrm{H}$ & -4.60116 & -2.46994 & 3.03609 \\
\hline $\mathrm{C}$ & -4.73079 & 0.77037 & 3.36399 \\
\hline $\mathrm{H}$ & -3.71737 & 0.92315 & 3.7546 \\
\hline $\mathrm{H}$ & -5.16509 & 1.75823 & 3.169 \\
\hline $\mathrm{H}$ & -5.31974 & 0.29823 & 4.16065 \\
\hline $\mathrm{C}$ & 3.21348 & -1.07279 & -0.50944 \\
\hline $\mathrm{C}$ & 3.61944 & -0.73719 & 0.91936 \\
\hline $\mathrm{C}$ & 3.80243 & -0.16452 & -1.60579 \\
\hline $\mathrm{C}$ & 6.05071 & -0.3597 & 0.29308 \\
\hline $\mathrm{C}$ & 5.17048 & -0.64433 & -2.11553 \\
\hline $\mathrm{C}$ & 6.06733 & -1.19214 & -0.99402 \\
\hline & & & \\
\hline $\mathrm{H}$ & & \\
\hline $\mathrm{H}$ & & \\
\hline $\mathrm{H}$ & & \\
\hline
\end{tabular}




\begin{tabular}{|c|c|c|c|}
$\mathrm{H}$ & 3.46946 & -2.12048 & -0.7183 \\
\hline $\mathrm{H}$ & 6.08518 & 0.71491 & 0.04875 \\
\hline $\mathrm{H}$ & 3.87841 & 0.8608 & -1.22069 \\
\hline $\mathrm{H}$ & 5.67971 & 0.18386 & -2.62498 \\
\hline $\mathrm{H}$ & 2.12382 & -1.01641 & -0.52367 \\
\hline $\mathrm{H}$ & 3.0973 & -0.11682 & -2.44352 \\
\hline $\mathrm{H}$ & 6.96996 & -0.5745 & 0.847 \\
\hline $\mathrm{H}$ & 5.03048 & -1.4302 & -2.86892 \\
\hline $\mathrm{H}$ & 7.1036 & -1.23148 & -1.35108 \\
\hline $\mathrm{H}$ & 5.7949 & -2.22411 & -0.74332 \\
\hline $\mathrm{C}$ & 5.31785 & -0.28484 & 2.62963 \\
\hline $\mathrm{H}$ & 6.30144 & -0.72137 & 2.83738 \\
\hline $\mathrm{H}$ & 5.42177 & 0.8072 & 2.73273 \\
\hline
\end{tabular}

\begin{tabular}{|c|c|c|c|}
$\mathrm{C}$ & 2.90332 & -0.33223 & 3.1709 \\
\hline $\mathrm{H}$ & 2.11378 & -0.80335 & 3.76792 \\
\hline $\mathrm{H}$ & 2.80703 & 0.75183 & 3.33362 \\
\hline $\mathrm{C}$ & 4.28167 & -0.81089 & 3.61388 \\
\hline $\mathrm{H}$ & 4.51988 & -0.46431 & 4.6255 \\
\hline $\mathrm{H}$ & 4.30786 & -1.90738 & 3.62358 \\
\hline $\mathrm{N}$ & 2.63959 & -0.62954 & 1.76452 \\
\hline $\mathrm{N}$ & 4.95817 & -0.63098 & 1.24624 \\
\hline
\end{tabular}

Table 5.2-8: Atomic coordinates of structure 17c-TS-enolate

\begin{tabular}{|c|c|c|c|}
\hline \multicolumn{4}{|c|}{ 17c-TS-enolate } \\
\hline atom & $\mathrm{X}$ & $\mathrm{Y}$ & $\mathrm{Z}$ \\
\hline $\mathrm{C}$ & 2.9039 & 1.77208 & 2.01498 \\
\hline $\mathrm{C}$ & 1.37736 & 1.86141 & 1.92022 \\
\hline $\mathrm{C}$ & 0.54591 & 0.59372 & 1.91198 \\
\hline $\mathrm{C}$ & 0.65464 & -0.40293 & 0.94831 \\
\hline $\mathrm{C}$ & 1.69855 & -0.34339 & -0.14802 \\
\hline $\mathrm{C}$ & 2.95056 & 0.16106 & -0.0585 \\
\hline $\mathrm{C}$ & 3.65625 & 0.95488 & 0.97611 \\
\hline $\mathrm{O}$ & -0.0717 & -1.48265 & 0.93781 \\
\hline $\mathrm{C}$ & 3.7735 & 0.62579 & 2.48015 \\
\hline $\mathrm{H}$ & 4.5559 & 1.41868 & 0.57222 \\
\hline $\mathrm{H}$ & 3.3454 & 2.75295 & 2.20956 \\
\hline $\mathrm{C}$ & 5.09536 & 1.04087 & 3.11696 \\
\hline $\mathrm{C}$ & 3.26535 & -0.68551 & 3.05257 \\
\hline $\mathrm{C}$ & -0.22167 & 0.29984 & 3.18422 \\
\hline $\mathrm{H}$ & 1.03514 & 2.47809 & 2.76453 \\
\hline $\mathrm{H}$ & 1.19068 & 2.47872 & 1.02483 \\
\hline $\mathrm{H}$ & -1.42569 & 0.58227 & 0.94095 \\
\hline $\mathrm{H}$ & 4.9762 & 1.19033 & 4.19906 \\
\hline $\mathrm{H}$ & 5.86132 & 0.26721 & 2.97282 \\
\hline $\mathrm{H}$ & 5.47927 & 1.9758 & 2.69265 \\
\hline $\mathrm{H}$ & 4.06116 & -1.44099 & 3.0034 \\
\hline $\mathrm{H}$ & 3.00062 & -0.56174 & 4.11148 \\
\hline $\mathrm{H}$ & 2.39443 & -1.07552 & 2.53154 \\
\hline & & & \\
\hline
\end{tabular}

\begin{tabular}{|c|c|c|c|}
\hline$H$ & -0.65199 & -0.70444 & 3.13594 \\
\hline$H$ & -1.04044 & 1.01166 & 3.39342 \\
\hline$H$ & 0.4366 & 0.34193 & 4.06809 \\
\hline $\mathrm{C}$ & 0.57302 & 4.86358 & -1.02982 \\
\hline$H$ & 0.75558 & 5.76266 & -0.44683 \\
\hline$H$ & -0.36757 & 4.34843 & -0.85577 \\
\hline $\mathrm{C}$ & 0.74713 & 2.02591 & -2.24045 \\
\hline $\mathrm{H}$ & 0.47298 & 1.95346 & -1.18894 \\
\hline $\mathrm{C}$ & 1.45941 & 4.41665 & -1.93392 \\
\hline $\mathrm{C}$ & 1.25189 & 3.17697 & -2.7102 \\
\hline $\mathrm{H}$ & 1.59391 & 3.20844 & -3.74791 \\
\hline $\mathrm{H}$ & 3.58835 & -0.03116 & -0.92259 \\
\hline $\mathrm{C}$ & 2.73082 & 5.17677 & -2.23853 \\
\hline $\mathrm{H}$ & 2.79971 & 6.10166 & -1.65786 \\
\hline $\mathrm{H}$ & 3.61589 & 4.56511 & -2.02133 \\
\hline $\mathrm{H}$ & 2.78363 & 5.43737 & -3.30442 \\
\hline $\mathrm{C}$ & 0.54022 & 0.78667 & -3.07565 \\
\hline $\mathrm{H}$ & 0.76367 & 1.06102 & -4.11676 \\
\hline $\mathrm{C}$ & 1.34348 & -1.1337 & -1.41249 \\
\hline $\mathrm{H}$ & 0.30524 & -1.45384 & -1.30316 \\
\hline $\mathrm{C}$ & 1.51174 & -0.37916 & -2.74566 \\
\hline $\mathrm{H}$ & 1.3878 & -1.1315 & -3.53604 \\
\hline $\mathrm{H}$ & 2.5422 & -0.01257 & -2.82876 \\
\hline $\mathrm{C}$ & -0.93726 & 0.34539 & -3.03048 \\
\hline $\mathrm{H}$ & -1.588 & 1.12833 & -3.43735 \\
\hline & & & \\
\hline $\mathrm{H}$ & & \\
\hline $\mathrm{H}$ & & \\
\hline $\mathrm{H}$ & & \\
\hline $\mathrm{H}$ & & & \\
\hline $\mathrm{H}$ & & &
\end{tabular}




\begin{tabular}{|c|c|c|c|}
$\mathrm{H}$ & -1.09082 & -0.56645 & -3.62043 \\
\hline $\mathrm{H}$ & -1.2629 & 0.13894 & -2.00463 \\
\hline $\mathrm{O}$ & 2.17276 & -2.31064 & -1.54987 \\
\hline $\mathrm{Si}$ & 1.87849 & -3.83816 & -0.9245 \\
\hline $\mathrm{C}$ & 3.05907 & -4.95218 & -1.89386 \\
\hline $\mathrm{H}$ & 2.98299 & -5.99452 & -1.55857 \\
\hline $\mathrm{H}$ & 2.84214 & -4.92907 & -2.9685 \\
\hline $\mathrm{H}$ & 4.10024 & -4.63582 & -1.75806 \\
\hline $\mathrm{C}$ & 2.25434 & -3.97333 & 0.91728 \\
\hline $\mathrm{H}$ & 3.28319 & -3.66752 & 1.14117 \\
\hline $\mathrm{H}$ & 1.57059 & -3.32272 & 1.47234 \\
\hline $\mathrm{H}$ & 2.12494 & -5.00526 & 1.27034 \\
\hline $\mathrm{C}$ & 0.08983 & -4.36414 & -1.2415 \\
\hline $\mathrm{H}$ & -0.58979 & -3.71155 & -0.68355 \\
\hline $\mathrm{H}$ & -0.1707 & -4.31257 & -2.30595 \\
\hline $\mathrm{H}$ & -0.07797 & -5.39568 & -0.90575 \\
\hline $\mathrm{C}$ & -3.05655 & -1.26859 & 0.37351 \\
\hline $\mathrm{C}$ & -3.39825 & 0.19084 & 0.5641 \\
\hline $\mathrm{C}$ & -4.00924 & -2.26096 & 1.06184 \\
\hline $\mathrm{C}$ & -5.88749 & -0.11297 & 0.33207 \\
\hline $\mathrm{C}$ & -5.24102 & -2.60388 & 0.21124 \\
\hline $\mathrm{C}$ & -5.80717 & -1.37989 & -0.5232 \\
\hline $\mathrm{H}$ & -3.01785 & -1.46534 & -0.70662 \\
\hline $\mathrm{H}$ & -6.2692 & -0.34593 & 1.33564 \\
\hline & & & \\
\hline
\end{tabular}

\begin{tabular}{|c|c|c|c|}
$\mathrm{H}$ & -4.31652 & -1.85642 & 2.03482 \\
\hline $\mathrm{H}$ & -6.01647 & -3.04502 & 0.84998 \\
\hline $\mathrm{H}$ & -2.0235 & -1.40114 & 0.73284 \\
\hline $\mathrm{H}$ & -3.44581 & -3.1728 & 1.28506 \\
\hline $\mathrm{H}$ & -6.60737 & 0.56945 & -0.1264 \\
\hline $\mathrm{H}$ & -4.97894 & -3.36722 & -0.53167 \\
\hline $\mathrm{H}$ & -6.82497 & -1.59854 & -0.86694 \\
\hline $\mathrm{H}$ & -5.22711 & -1.15886 & -1.42672 \\
\hline $\mathrm{C}$ & -4.88676 & 2.1216 & 0.74153 \\
\hline $\mathrm{H}$ & -5.7714 & 2.41527 & 0.17169 \\
\hline $\mathrm{H}$ & -5.12156 & 2.25075 & 1.80646 \\
\hline $\mathrm{C}$ & -2.44303 & 2.4365 & 1.02257 \\
\hline $\mathrm{H}$ & -1.52877 & 2.87253 & 0.6138 \\
\hline $\mathrm{H}$ & -2.45641 & 2.6481 & 2.0988 \\
\hline $\mathrm{C}$ & -3.68965 & 2.97513 & 0.3396 \\
\hline $\mathrm{H}$ & -3.86671 & 4.01458 & 0.62988 \\
\hline $\mathrm{H}$ & -3.56497 & 2.94751 & -0.7486 \\
\hline $\mathrm{N}$ & -2.36864 & 0.9897 & 0.81919 \\
\hline $\mathrm{N}$ & -4.64266 & 0.68651 & 0.47145 \\
\hline & & &
\end{tabular}

Table 5.2-9: Atomic coordinates of structure 17c

\begin{tabular}{|c|c|c|c|}
\hline \multicolumn{4}{|c|}{$17 c$} \\
\hline atom & $\mathrm{X}$ & $\mathrm{Y}$ & $\mathrm{Z}$ \\
\hline $\mathrm{C}$ & 0.07463 & -2.87254 & 0.44473 \\
\hline $\mathrm{C}$ & 0.49319 & -2.86256 & -1.01372 \\
\hline $\mathrm{C}$ & 0.24122 & -1.48663 & -1.68658 \\
\hline $\mathrm{C}$ & 1.42462 & -0.53648 & -1.4696 \\
\hline $\mathrm{C}$ & 1.94551 & -0.20823 & -0.10451 \\
\hline $\mathrm{C}$ & 1.56479 & -0.76921 & 1.06445 \\
\hline $\mathrm{C}$ & 0.57305 & -1.81411 & 1.39767 \\
\hline $\mathrm{O}$ & 1.9575 & -0.01544 & -2.45038 \\
\hline $\mathrm{C}$ & 0.97072 & -3.28825 & 1.59699 \\
\hline $\mathrm{H}$ & -0.1437 & -1.45974 & 2.14062 \\
\hline $\mathrm{H}$ & -0.97791 & -3.1218 & 0.57888 \\
\hline $\mathrm{C}$ & 0.31395 & -4.0193 & 2.75656 \\
\hline
\end{tabular}

\begin{tabular}{|c|c|c|c|}
$\mathrm{C}$ & 2.40446 & -3.72272 & 1.34761 \\
\hline $\mathrm{C}$ & -0.06282 & -1.63724 & -3.18165 \\
\hline $\mathrm{H}$ & 1.54356 & -3.14779 & -1.14011 \\
\hline $\mathrm{H}$ & -0.087 & -3.62362 & -1.54964 \\
\hline $\mathrm{H}$ & -0.63223 & -1.02625 & -1.1998 \\
\hline $\mathrm{H}$ & 0.29471 & -5.10181 & 2.57337 \\
\hline $\mathrm{H}$ & 0.86298 & -3.85145 & 3.69208 \\
\hline $\mathrm{H}$ & -0.7194 & -3.68818 & 2.90979 \\
\hline $\mathrm{H}$ & 2.44093 & -4.77204 & 1.02734 \\
\hline $\mathrm{H}$ & 2.90488 & -3.11748 & 0.58764 \\
\hline $\mathrm{H}$ & 2.99263 & -3.63886 & 2.27 \\
\hline $\mathrm{H}$ & -0.96615 & -2.24307 & -3.31512 \\
\hline $\mathrm{H}$ & 0.76231 & -2.12922 & -3.70856 \\
\hline $\mathrm{H}$ & -0.22122 & -0.66603 & -3.65787 \\
\hline
\end{tabular}




\begin{tabular}{|c|c|c|c|}
$\mathrm{C}$ & -0.07454 & 3.92862 & 2.73552 \\
\hline $\mathrm{H}$ & -0.21008 & 4.65173 & 3.53651 \\
\hline $\mathrm{H}$ & 0.82471 & 3.32256 & 2.77621 \\
\hline $\mathrm{C}$ & 0.19693 & 2.50862 & -0.0947 \\
\hline $\mathrm{H}$ & 0.0276 & 1.79052 & -0.89633 \\
\hline $\mathrm{C}$ & -0.99452 & 3.77954 & 1.76806 \\
\hline $\mathrm{C}$ & -0.87888 & 2.81853 & 0.65154 \\
\hline $\mathrm{H}$ & -1.81413 & 2.33579 & 0.36219 \\
\hline $\mathrm{H}$ & 2.08087 & -0.38445 & 1.94605 \\
\hline $\mathrm{C}$ & -2.27826 & 4.58279 & 1.78412 \\
\hline $\mathrm{H}$ & -2.33032 & 5.24767 & 2.65185 \\
\hline $\mathrm{H}$ & -2.37563 & 5.19216 & 0.87616 \\
\hline $\mathrm{H}$ & -3.15269 & 3.91816 & 1.80888 \\
\hline $\mathrm{C}$ & 1.58937 & 3.08805 & -0.03078 \\
\hline $\mathrm{H}$ & 1.5459 & 3.98242 & 0.60335 \\
\hline $\mathrm{C}$ & 3.04701 & 0.85849 & -0.05705 \\
\hline $\mathrm{H}$ & 3.32767 & 1.08296 & -1.0885 \\
\hline $\mathrm{C}$ & 2.64126 & 2.16764 & 0.64865 \\
\hline $\mathrm{H}$ & 3.56479 & 2.75134 & 0.75644 \\
\hline $\mathrm{H}$ & 2.30839 & 1.92134 & 1.665 \\
\hline $\mathrm{C}$ & 2.04004 & 3.5322 & -1.43789 \\
\hline $\mathrm{H}$ & 1.37146 & 4.3075 & -1.82865 \\
\hline $\mathrm{H}$ & 3.05604 & 3.94538 & -1.41055 \\
\hline $\mathrm{H}$ & 2.03271 & 2.69709 & -2.14688 \\
\hline $\mathrm{O}$ & 4.18615 & 0.34701 & 0.66294 \\
\hline $\mathrm{Si}$ & 5.61988 & -0.11992 & -0.08226 \\
\hline $\mathrm{C}$ & 6.71391 & -0.73333 & 1.32122 \\
\hline $\mathrm{H}$ & 7.68906 & -1.06465 & 0.94339 \\
\hline $\mathrm{H}$ & 6.89272 & 0.05727 & 2.05914 \\
\hline $\mathrm{H}$ & 6.25226 & -1.57982 & 1.84261 \\
\hline $\mathrm{C}$ & 5.30659 & -1.49073 & -1.34365 \\
\hline $\mathrm{H}$ & 4.94361 & -2.40396 & -0.85667 \\
\hline $\mathrm{H}$ & 4.55857 & -1.18627 & -2.08523 \\
\hline $\mathrm{H}$ & 6.22707 & -1.74515 & -1.88422 \\
\hline & & & \\
\hline
\end{tabular}

\begin{tabular}{|c|c|c|c|} 
C & 6.41973 & 1.35802 & -0.9471 \\
\hline$H$ & 5.77556 & 1.7703 & -1.73307 \\
\hline$H$ & 6.63671 & 2.16409 & -0.23608 \\
\hline$H$ & 7.36595 & 1.06663 & -1.42045 \\
\hline C & -3.62546 & -0.58731 & 0.95824 \\
\hline C & -4.00994 & -0.14305 & -0.44819 \\
\hline C & -4.51621 & -0.0611 & 2.09978 \\
\hline C & -6.48839 & -0.53787 & -0.04623 \\
\hline C & -5.72935 & -0.96053 & 2.38526 \\
\hline C & -6.36231 & -1.53816 & 1.10913 \\
\hline$H$ & -3.58611 & -1.68532 & 0.99604 \\
\hline$H$ & -6.83031 & 0.43908 & 0.33699 \\
\hline$H$ & -4.84828 & 0.95641 & 1.85476 \\
\hline$H$ & -6.48153 & -0.38947 & 2.94494 \\
\hline$H$ & -2.60077 & -0.23348 & 1.08545 \\
\hline$H$ & -3.91321 & 0.03448 & 3.01041 \\
\hline$H$ & -7.27935 & -0.89285 & -0.71526 \\
\hline$H$ & -5.42897 & -1.79256 & 3.03554 \\
\hline$H$ & -7.36827 & -1.90665 & 1.34478 \\
\hline$H$ & -5.79747 & -2.40741 & 0.752 \\
\hline C & -5.65262 & 0.11464 & -2.24806 \\
\hline$H$ & -6.46459 & -0.51407 & -2.63266 \\
\hline$H$ & -6.04313 & 1.14479 & -2.19437 \\
\hline C & -3.2876 & 0.80169 & -2.51416 \\
\hline$H$ & -2.36192 & 0.65802 & -3.08421 \\
\hline$H$ & -3.4824 & 1.88548 & -2.52019 \\
\hline C & -4.44642 & 0.06 & -3.17635 \\
\hline$H$ & -4.70209 & 0.49775 & -4.14815 \\
\hline$H$ & -4.16608 & -0.98672 & -3.34863 \\
\hline N & -3.04507 & 0.37011 & -1.14095 \\
\hline N & -5.30466 & -0.3717 & -0.90648 \\
\hline & & & \\
\hline$H$
\end{tabular}

\subsection{Summary of DFT Calculations - Figure 3 Curtin-Hammett}




\begin{tabular}{|c|c|c|c|c|c|c|c|c|c|c|c|}
\hline \multicolumn{4}{|c|}{ Diels-Alder route } & \multicolumn{4}{|c|}{ Epimerization with DBU } & \multicolumn{4}{|c|}{ Energies for the Curtin-Hammet Scheme (only) } \\
\hline \multirow{3}{*}{ Gibbs Free Energies (G) } & & & & & & & & (left to right) & & \multirow[b]{3}{*}{$\mathrm{kcal} / \mathrm{mol}$} & \multirow[b]{3}{*}{ kcal } \\
\hline & & & & Gibbs Free Energies (G) & & & & \multirow{2}{*}{\multicolumn{2}{|c|}{ Gibbs Free Energies (G) }} & & \\
\hline & hartree & $\mathrm{kcal} / \mathrm{mol}$ & kcal & & hartree & $\mathrm{kcal} / \mathrm{mol}$ & kcal & & & & \\
\hline $17 \mathrm{c}$ & $-1338,781284$ & $-8,40098 E+05$ & $-1,39502 \mathrm{E}-18$ & $17 \mathrm{c}$ & $-1800,641060$ & $-1,12992 E+06$ & $-1,87628 \mathrm{E}-18$ & epi-19 & $-1338,811292$ & \begin{tabular}{l|l}
2 & $-840116,7696$ \\
\end{tabular} & \begin{tabular}{|l|l|}
5 & $-1,39505 E-18$ \\
\end{tabular} \\
\hline 17 CTS19 & $-1338,729617$ & $-8,40066 E+05$ & $-1,39496 \mathrm{E}-18$ & 17c-TS-enolat & $-1800,59763$ & $3-1,12989 E+06$ & $-1,87623 \mathrm{E}-18$ & 17b-TS-epi -19 & $-1338,714577$ & $-840056,08$ & $-1,39495 \mathrm{E}-18$ \\
\hline \multirow[t]{2}{*}{19} & $-1338,805688$ & $-8,40113 E+05$ & $-1,39504 \mathrm{E}-18$ & 17c-enolat & $-1800,632960$ & $-1,12991 E+06$ & $-1,87627 \mathrm{E}-18$ & $17 \mathrm{~b}$ & $-1338,780715$ & $-840097,5823$ & $-1,39501 \mathrm{E}-18$ \\
\hline & & & & & & & & 17b-TS-enolat & $-1338,726945$ & $-840063,8411$ & $-1,39496 \mathrm{E}-18$ \\
\hline 17b & $-1338,780715$ & $-8,40098 E+05$ & $-1,39501 \mathrm{E}-18$ & $17 \mathrm{~b}$ & $-1800,647680$ & $-1,12992 E+06$ & $-1,87628 \mathrm{E}-18$ & 17b-enolat & $-1338,761765$ & $-840085,691$ & $1-1,3949951 E-18$ \\
\hline 17bTSepi-19 & $-1338,714577$ & $-8,40056 \mathrm{E}+05$ & $-1,39495 \mathrm{E}-18$ & 17b-TS-enolat & $-1800,59391$ & $1-1,12989 E+06$ & $-1,87623 \mathrm{E}-18$ & enol & $-1338,763925$ & $-840087,0464$ & $-1,3949974 E-18$ \\
\hline \multirow[t]{5}{*}{ epi-19 } & $-1338,811292$ & $-8,40117 E+05$ & $-1,39505 E-18$ & 17b-enolat & $-1800,628730$ & $-1,12991 E+06$ & $-1,87626 \mathrm{E}-18$ & 17c-enolat & $-1338,765995$ & $-840088,3453$ & $3-1,3949995 E-18$ \\
\hline & & & & & & & & 17c-TS-enolat & $-1338,737854$ & $-840070,6866$ & $-1,39497 \mathrm{E}-18$ \\
\hline & & & & enol & $-1800,63089$ & a $-1,12991 \mathrm{E}+06$ & $-1,87626 \mathrm{E}-18$ & $17 \mathrm{c}$ & $-1338,781284$ & $-840097,9393$ & $-1,39502 \mathrm{E}-18$ \\
\hline & & & & & & & & 17c-TS-19 & $-1338,729617$ & $-840065,5178$ & $-1,39496 \mathrm{E}-18$ \\
\hline & & & & & & & & 19 & $-1338,805688$ & $-840113,2531$ & $-1,39504 \mathrm{E}-18$ \\
\hline \multicolumn{2}{|c|}{ Gibbs Free Energy differences $(\Delta G)$} & & & Gibbs Free Energy differences $(\Delta G)$ & & & & & & & \\
\hline & hartree & $\mathrm{kcal} / \mathrm{mol}$ & kcal & & hartree & $\mathrm{kcal} / \mathrm{mol}$ & kcal & \multicolumn{2}{|c|}{ Gibbs Free Energy differences $(\Delta G)$} & & \\
\hline $17 \mathrm{c}-\mathrm{TS}$ & 0,051667 & $3,24215 \mathrm{E}+01$ & $5,38372 \mathrm{E}-23$ & $17 \mathrm{c}-\mathrm{TS}$ & 0,043430 & $2,72527 E+01$ & $4,52542 \mathrm{E}-23$ & & hartree & $\mathrm{kcal} / \mathrm{mol}$ & kcal \\
\hline TS-19 & 0,076071 & $4,77353 \mathrm{E}+01$ & $7,92663 \mathrm{E}-23$ & TS-enolat & 0,035330 & $2,21699 \mathrm{E}+01$ & $3,68140 E-23$ & epi-19-(17b-TS-epi-19) & 0,096715 & \begin{tabular}{l|l}
5 & 60,68957878 \\
\end{tabular} & $3 \quad 1,00777 E-22$ \\
\hline \multirow[t]{2}{*}{$17 \mathrm{c}-19$} & 0,024404 & $1,53137 \mathrm{E}+01$ & $2,54291 E-23$ & 17cenolat & 0,008100 & $5,08283 E+00$ & 8,44023E-24 & (17b-TS-epi-19)-17b & 0,066138 & 41,50222159 & $6,89161 \mathrm{E}-23$ \\
\hline & & & & & & & & 17b-(17b-TS-enolat) & 0,053770 & 33,74118442 & $5,60286 \mathrm{E}-23$ \\
\hline $17 \mathrm{~b}-T S$ & 0,066138 & $4,15022 E+01$ & $6,89161 \mathrm{E}-23$ & $17 \mathrm{~b}-\mathrm{TS}$ & 0,053770 & $3,37412 E+01$ & $5,60286 \mathrm{E}-23$ & (17b-TS-enolat)-(17b-enolat) & 0,034820 & 21,84987988 & $3,62826 \mathrm{E}-23$ \\
\hline TS-epi-19 & 0,096715 & $6,06896 \mathrm{E}+01$ & $1,00777 \mathrm{E}-22$ & TS-enolat & 0,034820 & $2,18499 \mathrm{E}+01$ & $3,62826 \mathrm{E}-23$ & (17b-enolat)-enol & 0,002160 & 1,355420464 & $2,25073 E-24$ \\
\hline \multirow[t]{2}{*}{ 17b-epi-19 } & 0,030577 & $1,91874 \mathrm{E}+01$ & $3,18614 \mathrm{E}-23$ & 17b-enolat & 0,018950 & $1,18913 E+01$ & $1,97460 \mathrm{E}-23$ & enol-(17c-enolat) & 0,002070 & 1,298944611 & $2,15695 \mathrm{E}-24$ \\
\hline & & & & & & & & (17c-enolat)-(17c-TS-enolat) & 0,028141 & 17,65874411 & $2,9323 E-23$ \\
\hline $17 c-17 b$ & 0,000569 & $3,57053 \mathrm{E}-01$ & $5,92900 E-25$ & $17 \mathrm{c}-17 \mathrm{~b}$ & 0,006620 & $4,15411 \mathrm{E}+00$ & 6,89807E-24 & (17c-TS-enolat)-17c & 0,043430 & 27,25273646 & $4,52542 E-23$ \\
\hline 17 cTS19- & & & & (17c-TS-enolat) - & & & & & & & \\
\hline 17bTSepi-19 & 0,01504 & $9,43774 E+00$ & $1,56717 \mathrm{E}-23$ & (17b-TS-enolat) & 0,00372 & $2,33434 E+00$ & $3,87625 \mathrm{E}-24$ & $17 c-(17 c-$ TS-19) & 0,051667 & 32,42153199 & $5,38372 E-23$ \\
\hline \multirow{4}{*}{19 -epi19 } & 0,005604 & $3,51656 \mathrm{E}+00$ & $5,83939 E-24$ & $17 c$ enol & 0,010170 & $6,38177 E+00$ & $1,05972 \mathrm{E}-23$ & $(17 \mathrm{C}-\mathrm{TS}-19)-19$ & 0,076071 & 47,7352732 & $7,92663 \mathrm{E}-23$ \\
\hline & & & & 17benol & 0,016790 & $1,05359 \mathrm{E}+01$ & $1,74952 \mathrm{E}-23$ & & & & \\
\hline & & & & (17c-enolat)-enol & 0,002070 & $1,29894 E+00$ & $2,15695 \mathrm{E}-24$ & & & & \\
\hline & & & & (17b-enolat)-enol & 0,002160 & $1,35542 \mathrm{E}+00$ & $2,25073 \mathrm{E}-24$ & & & & \\
\hline
\end{tabular}

\begin{tabular}{|l|r||r|r|}
\hline \multicolumn{1}{|l|}{ Activation barrier for Diels-Alder } & \multicolumn{1}{l|}{ vs. } & \\
\hline & \multicolumn{1}{|c|}{ hartree } & kcal/mol & \multicolumn{1}{l|}{ kcal } \\
\hline $\mathbf{1 7 c} \rightarrow$ TS & 0,051667 & 32,421532 & $5,38 \mathrm{E}-23$ \\
\hline $\mathbf{1 7 b} \rightarrow$ TS & 0,066138 & 41,502222 & $6,89 \mathrm{E}-23$ \\
\hline & & & \\
\hline Difference in Energy between the transition states & & \\
\hline a) between Diels-Alder TS & & & \\
\hline & & & \\
\hline $\mathbf{1 7 c T S 1 9 - ( 1 7 b T S e p i - 1 9 )}$ & 0,01504 & 9,437742489 & $1,56717 \mathrm{E}-23$ \\
\hline
\end{tabular}

\begin{tabular}{|l|r|r|r|}
\hline \multicolumn{2}{|l|}{ Activation barrier for deprotonation } & \multicolumn{2}{l|}{} \\
\hline $\mathbf{1 7 c} \rightarrow$ TS & hartree & $\mathrm{kcal} / \mathrm{mol}$ & $\mathrm{kcal}$ \\
\hline $\mathbf{1 7 b} \rightarrow$ TS & 0,043430 & 27,252736 & $4,53 \mathrm{E}-23$ \\
\hline
\end{tabular}

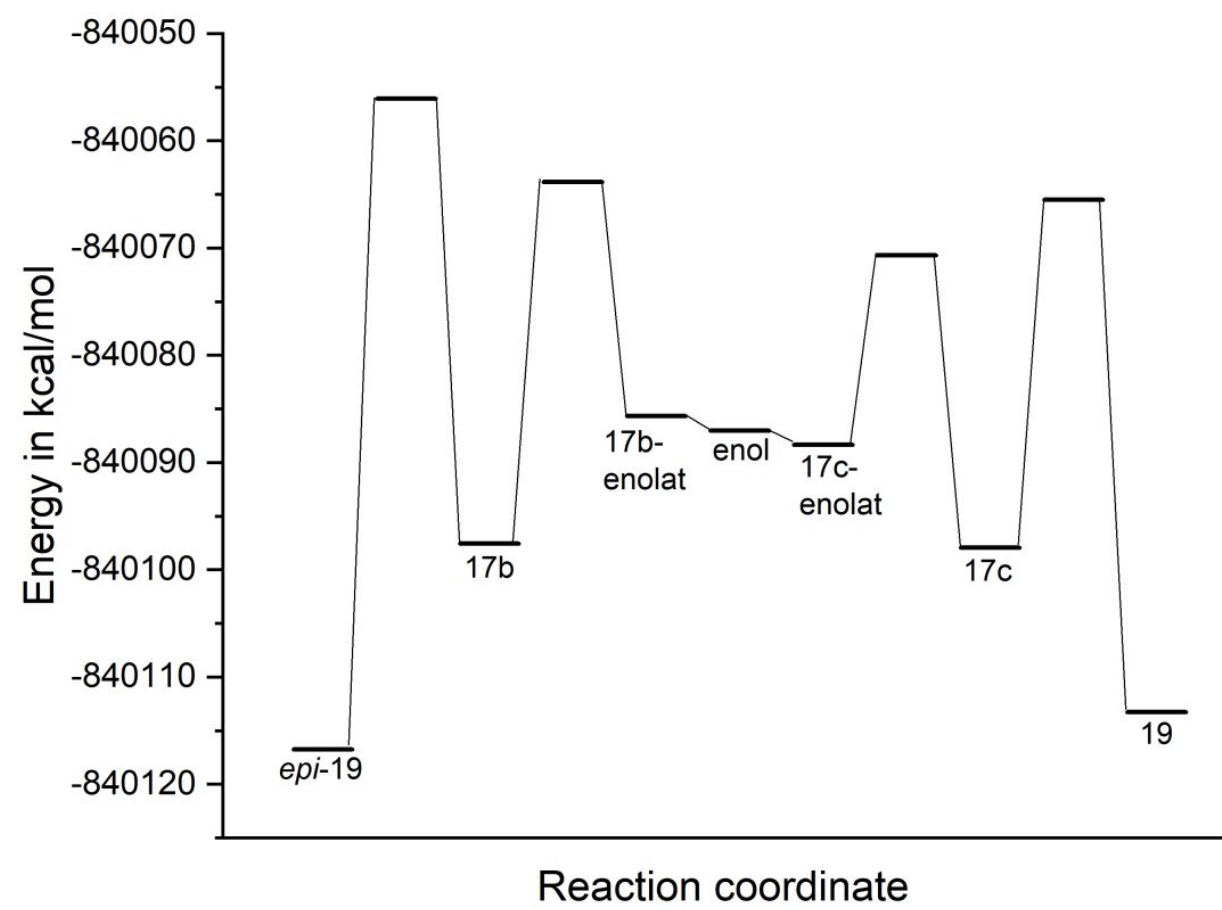


6. Single Crystal X-ray Diffraction Analysis

X-ray Crystal Structure for SI-3

CCDC number 2041811
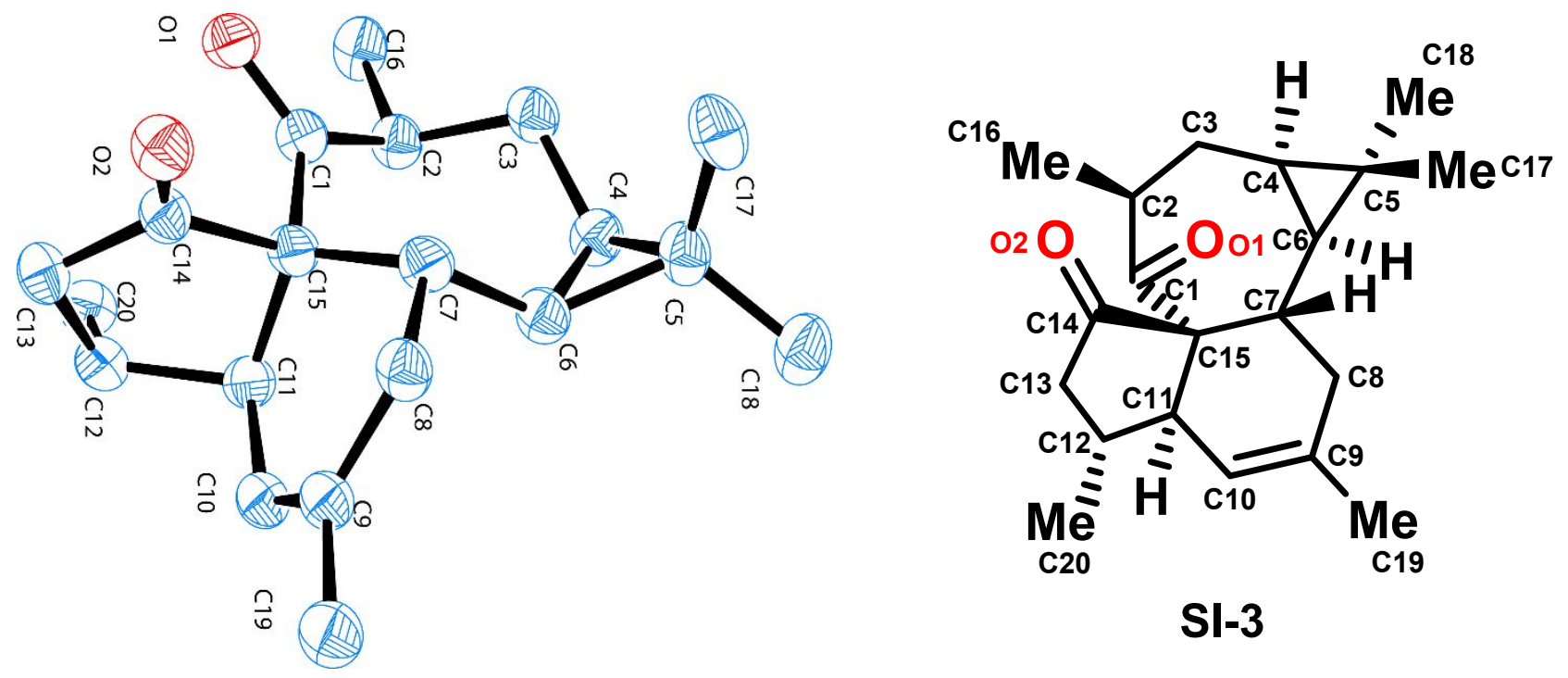

Figure 1: ORTEP representation of SI-3 (left) and 2D representation of SI-3 (right) with defined stereocenters. 
Table 1: Crystal data and structure refinement of SI-3.

\begin{tabular}{|c|c|}
\hline Identification code & CCDC2041811 \\
\hline Empirical formula & $\mathrm{C}_{20} \mathrm{H}_{28} \mathrm{O}_{2}$ \\
\hline Formula weight & 300.42 \\
\hline Temperature/K & 100 \\
\hline Crystal system & monoclinic \\
\hline Space group & $\mathrm{P} 2_{1}$ \\
\hline $\mathrm{a} / \AA$ & $6.5200(17)$ \\
\hline $\mathrm{b} / \AA \AA$ & $17.715(3)$ \\
\hline$c / \AA$ & $7.7507(19)$ \\
\hline$\alpha /^{\circ}$ & 90 \\
\hline$\beta /^{\circ}$ & $100.47(2)$ \\
\hline$y /^{\circ}$ & 90 \\
\hline Volume $/ \AA^{3}$ & $880.3(3)$ \\
\hline Z & 2 \\
\hline$\rho_{\text {calc }} \mathrm{g} / \mathrm{cm}^{3}$ & 1.133 \\
\hline$\mu / \mathrm{mm}^{-1}$ & 0.071 \\
\hline$F(000)$ & 328.0 \\
\hline Crystal size $/ \mathrm{mm}^{3}$ & $0.8 \times 0.533 \times 0.3$ \\
\hline Radiation & $\operatorname{MoKa}(\lambda=0.71073)$ \\
\hline $2 \Theta$ range for data collection ${ }^{\circ}$ & 5.818 to 58.732 \\
\hline Index ranges & $-8 \leq h \leq 8,-23 \leq k \leq 24,-10 \leq \mathrm{I} \leq 10$ \\
\hline Reflections collected & 9807 \\
\hline Independent reflections & $4718\left[R_{\text {int }}=0.0753, R_{\text {sigma }}=0.0632\right]$ \\
\hline
\end{tabular}


Data/restraints/parameters

Goodness-of-fit on $\mathrm{F}^{2}$

Final $R$ indexes $[I>=2 \sigma(I)]$

Final $\mathrm{R}$ indexes [all data]

Largest diff. peak/hole / e $\AA^{-3}$

Flack parameter
$4718 / 1 / 204$

1.042

$R_{1}=0.0749, w R_{2}=0.1911$

$\mathrm{R}_{1}=0.0891, \mathrm{wR}_{2}=0.2117$

$0.28 /-0.42$

$-0.4(10)$ 
Table 2: Fractional Atomic Coordinates $\left(\times 10^{4}\right)$ and Equivalent Isotropic Displacement Parameters $\left(\AA^{2} \times 10^{3}\right)$ for SI-3. $U_{\text {eq }}$ is defined as $1 / 3$ of of the trace of the orthogonalized $U_{I J}$ tensor.

\begin{tabular}{|c|c|c|c|c|}
\hline Atom & $x$ & $y$ & $z$ & $\mathrm{U}(\mathrm{eq})$ \\
\hline 01 & $-341(4)$ & $3682.0(17)$ & $5096(4)$ & $35.3(6)$ \\
\hline $\mathrm{O} 2$ & 1318(5) & $4540.0(17)$ & $8462(4)$ & $36.1(6)$ \\
\hline C1 & $741(6)$ & $4107(2)$ & $4403(5)$ & $29.7(7)$ \\
\hline $\mathrm{C} 2$ & $409(6)$ & $4161(2)$ & $2415(5)$ & $31.8(7)$ \\
\hline C3 & $-759(6)$ & $4907(2)$ & $1848(5)$ & $33.0(7)$ \\
\hline C4 & $747(6)$ & $5556(2)$ & 1926(5) & $32.3(7)$ \\
\hline C5 & $438(7)$ & $6333(2)$ & $2651(5)$ & $34.0(8)$ \\
\hline C6 & $2063(6)$ & $5797(2)$ & $3659(5)$ & $31.6(7)$ \\
\hline C7 & 1798(6) & $5459(2)$ & $5413(5)$ & $30.8(7)$ \\
\hline C8 & $3210(6)$ & $5902(2)$ & $6866(5)$ & $32.2(7)$ \\
\hline C9 & $5480(6)$ & $5670(2)$ & 7089(5) & $33.0(8)$ \\
\hline C10 & $6073(6)$ & $5037(2)$ & $6401(5)$ & $33.9(8)$ \\
\hline C11 & $4643(6)$ & $4443(2)$ & $5408(5)$ & $30.5(7)$ \\
\hline C12 & $5181(6)$ & $3661(2)$ & $6296(5)$ & $33.6(7)$ \\
\hline C13 & $3918(6)$ & $3652(2)$ & $7801(5)$ & $34.9(8)$ \\
\hline C14 & $2372(6)$ & $4297(2)$ & $7445(5)$ & $30.6(7)$ \\
\hline C15 & $2367(5)$ & $4605(2)$ & $5585(5)$ & $28.4(7)$ \\
\hline C16 & $-812(8)$ & $3476(2)$ & $1575(5)$ & $39.8(9)$ \\
\hline C17 & $-1514(7)$ & $6528(3)$ & $3358(6)$ & $40.0(9)$ \\
\hline C18 & $1242(8)$ & $6991(3)$ & $1719(6)$ & $46.4(10)$ \\
\hline C19 & $7024(7)$ & $6204(3)$ & $8135(6)$ & $41.4(9)$ \\
\hline $\mathrm{C} 20$ & 4696(7) & $3002(2)$ & $5007(6)$ & $37.4(8)$ \\
\hline
\end{tabular}

Table 3: Anisotropic Displacement Parameters $\left(\AA^{2} \times 10^{3}\right)$ for SI-3. The Anisotropic displacement factor exponent takes the form: $-2 \pi^{2}\left[h^{2} a^{*} U_{11}+2 h k a * b^{*} U_{12}+\ldots\right]$. 


\begin{tabular}{|c|c|c|c|c|c|c|}
\hline Atom & $U_{11}$ & $\mathbf{U}_{22}$ & $\mathbf{U}_{33}$ & $\mathbf{U}_{23}$ & $U_{13}$ & $U_{12}$ \\
\hline $\mathrm{O} 1$ & $33.6(13)$ & $43.5(15)$ & $27.4(12)$ & $1.5(11)$ & $1.8(10)$ & $-5.1(12)$ \\
\hline $\mathrm{O} 2$ & $38.6(14)$ & $44.3(16)$ & $25.0(12)$ & $-0.5(11)$ & $4.8(11)$ & $2.5(12)$ \\
\hline $\mathrm{C} 1$ & $31.7(17)$ & $30.8(16)$ & $26.0(15)$ & $-1.9(13)$ & $3.7(13)$ & $1.3(14)$ \\
\hline $\mathrm{C} 2$ & $35.3(17)$ & $32.3(16)$ & $25.8(15)$ & $-2.1(13)$ & $0.3(13)$ & $-1.4(14)$ \\
\hline C3 & $34.0(18)$ & $36.6(18)$ & $25.3(16)$ & $0.2(14)$ & $-2.9(14)$ & $-0.4(15)$ \\
\hline $\mathrm{C} 4$ & $37.6(18)$ & $35.2(18)$ & $23.1(15)$ & $0.9(13)$ & $3.4(13)$ & $-1.9(15)$ \\
\hline C5 & $41(2)$ & $32.7(18)$ & $24.4(16)$ & $0.9(13)$ & $-4.6(14)$ & $0.4(15)$ \\
\hline C6 & $33.3(18)$ & $33.4(17)$ & $26.1(16)$ & $-0.1(13)$ & $-0.3(13)$ & $-1.6(14)$ \\
\hline $\mathrm{C} 7$ & $31.1(16)$ & $35.5(18)$ & $24.1(15)$ & $-0.5(13)$ & $0.3(13)$ & $-2.2(14)$ \\
\hline C8 & $36.9(19)$ & $33.0(17)$ & $24.4(15)$ & $-0.1(13)$ & $-0.6(14)$ & $-0.5(14)$ \\
\hline $\mathrm{C9}$ & $33.9(18)$ & $36.7(19)$ & $25.3(16)$ & $2.9(13)$ & $-3.1(13)$ & $-3.3(14)$ \\
\hline C10 & $32.6(18)$ & $36.1(19)$ & $30.1(17)$ & $3.9(14)$ & $-1.6(14)$ & $-1.3(14)$ \\
\hline C11 & $30.6(17)$ & $34.5(18)$ & $25.9(15)$ & $1.0(13)$ & $4.3(13)$ & $-1.9(13)$ \\
\hline $\mathrm{C} 12$ & $31.0(16)$ & $35.8(18)$ & $32.5(18)$ & $0.7(15)$ & $2.2(14)$ & $1.3(15)$ \\
\hline C13 & $36.5(18)$ & $39.4(19)$ & $26.5(16)$ & $3.5(14)$ & $-0.3(14)$ & $2.8(16)$ \\
\hline C14 & $31.3(17)$ & $34.3(18)$ & $24.0(15)$ & $1.9(13)$ & $-0.8(13)$ & $-1.1(13)$ \\
\hline C15 & $28.5(16)$ & $33.9(17)$ & $21.6(14)$ & $0.4(12)$ & $1.3(12)$ & $-1.5(13)$ \\
\hline C16 & $52(2)$ & $36.0(19)$ & $27.5(17)$ & $-3.4(14)$ & $-2.4(16)$ & $-4.9(16)$ \\
\hline C17 & $46(2)$ & $40(2)$ & $29.9(18)$ & $-4.1(15)$ & $-6.4(16)$ & $10.0(17)$ \\
\hline C18 & $58(3)$ & $38(2)$ & $35(2)$ & $7.0(16)$ & $-12.5(19)$ & $-9.9(19)$ \\
\hline C19 & $45(2)$ & $41(2)$ & $33.1(19)$ & $0.4(16)$ & $-7.1(17)$ & $-6.8(17)$ \\
\hline $\mathrm{C} 20$ & $37(2)$ & $34.6(18)$ & $41(2)$ & $-3.3(15)$ & $6.7(17)$ & $1.8(16)$ \\
\hline
\end{tabular}

Table 4: Bond Lengths for SI-3. 


\begin{tabular}{llllll}
\hline Atom & Atom & Length/A & Atom & Atom & Length/A \\
O1 & $\mathrm{C} 1$ & $1.221(5)$ & $\mathrm{C} 7$ & $\mathrm{C} 8$ & $1.535(5)$ \\
$\mathrm{O} 2$ & $\mathrm{C} 14$ & $1.215(5)$ & $\mathrm{C} 7$ & $\mathrm{C} 15$ & $1.557(5)$ \\
$\mathrm{C} 1$ & $\mathrm{C} 2$ & $1.519(5)$ & $\mathrm{C} 8$ & $\mathrm{C} 9$ & $1.515(6)$ \\
$\mathrm{C} 1$ & $\mathrm{C} 15$ & $1.546(5)$ & $\mathrm{C} 9$ & $\mathrm{C} 10$ & $1.329(6)$ \\
$\mathrm{C} 2$ & $\mathrm{C} 3$ & $1.549(5)$ & $\mathrm{C} 9$ & $\mathrm{C} 19$ & $1.506(6)$ \\
$\mathrm{C} 2$ & $\mathrm{C} 16$ & $1.530(5)$ & $\mathrm{C} 10$ & $\mathrm{C} 11$ & $1.520(5)$ \\
$\mathrm{C} 3$ & $\mathrm{C} 4$ & $1.506(5)$ & $\mathrm{C} 11$ & $\mathrm{C} 12$ & $1.557(5)$ \\
$\mathrm{C} 4$ & $\mathrm{C} 5$ & $1.515(5)$ & $\mathrm{C} 11$ & $\mathrm{C} 15$ & $1.542(5)$ \\
$\mathrm{C} 4$ & $\mathrm{C} 6$ & $1.518(5)$ & $\mathrm{C} 12$ & $\mathrm{C} 13$ & $1.546(5)$ \\
$\mathrm{C} 5$ & $\mathrm{C} 6$ & $1.528(5)$ & $\mathrm{C} 12$ & $\mathrm{C} 20$ & $1.532(6)$ \\
$\mathrm{C} 5$ & $\mathrm{C} 17$ & $1.514(6)$ & $\mathrm{C} 13$ & $\mathrm{C} 14$ & $1.515(5)$ \\
$\mathrm{C} 5$ & $\mathrm{C} 18$ & $1.514(6)$ & $\mathrm{C} 14$ & $\mathrm{C} 15$ & $1.541(5)$ \\
$\mathrm{C} 6$ & $\mathrm{C} 7$ & $1.524(5)$ & & & \\
\hline
\end{tabular}

Table 5: Bond Angles for SI-3. 


\begin{tabular}{|c|c|c|c|c|c|c|c|}
\hline Atom & Atom & Atom & Angle $^{\circ}$ & Atom & Atom & Atom & Angle ${ }^{\circ}$ \\
\hline 01 & $\mathrm{C} 1$ & $\mathrm{C} 2$ & $119.8(3)$ & $\mathrm{C9}$ & $\mathrm{C} 8$ & $\mathrm{C7}$ & $112.8(3)$ \\
\hline 01 & $\mathrm{C} 1$ & C15 & $118.7(3)$ & C10 & $\mathrm{Cg}$ & $\mathrm{C} 8$ & $122.1(4)$ \\
\hline $\mathrm{C} 2$ & $\mathrm{C} 1$ & C15 & $121.4(3)$ & C10 & C9 & C19 & $122.1(4)$ \\
\hline $\mathrm{C} 1$ & $\mathrm{C} 2$ & C3 & $108.3(3)$ & C19 & $\mathrm{Cg}$ & $\mathrm{C} 8$ & $115.8(4)$ \\
\hline $\mathrm{C} 1$ & $\mathrm{C} 2$ & C16 & $110.4(3)$ & $\mathrm{Cg}$ & C10 & C11 & $126.3(4)$ \\
\hline C16 & $\mathrm{C} 2$ & C3 & $111.4(3)$ & C10 & C11 & C12 & $108.9(3)$ \\
\hline C4 & C3 & $\mathrm{C} 2$ & $110.9(3)$ & C10 & C11 & C15 & $109.7(3)$ \\
\hline C3 & $\mathrm{C} 4$ & C5 & $125.4(3)$ & C15 & C11 & C12 & $105.7(3)$ \\
\hline C3 & C4 & C6 & $120.7(3)$ & C13 & C12 & C11 & $103.5(3)$ \\
\hline C5 & $\mathrm{C} 4$ & C6 & $60.5(2)$ & $\mathrm{C} 20$ & C12 & C11 & $112.8(3)$ \\
\hline C4 & C5 & C6 & $59.8(2)$ & $\mathrm{C} 20$ & C12 & C13 & $114.1(3)$ \\
\hline C17 & C5 & $\mathrm{C} 4$ & $121.2(4)$ & C14 & C13 & C12 & $106.4(3)$ \\
\hline $\mathrm{C} 17$ & C5 & C6 & $120.5(3)$ & $\mathrm{O} 2$ & C14 & C13 & $125.8(3)$ \\
\hline $\mathrm{C} 17$ & C5 & C18 & $112.6(4)$ & $\mathrm{O} 2$ & C14 & C15 & $125.2(3)$ \\
\hline C18 & C5 & $\mathrm{C} 4$ & $116.2(4)$ & C13 & C14 & C15 & $109.0(3)$ \\
\hline C18 & C5 & C6 & $117.0(4)$ & $\mathrm{C} 1$ & C15 & $\mathrm{C7}$ & $112.1(3)$ \\
\hline $\mathrm{C} 4$ & C6 & C5 & $59.7(2)$ & C11 & C15 & $\mathrm{C} 1$ & 114.1(3) \\
\hline $\mathrm{C} 4$ & C6 & $\mathrm{C} 7$ & $122.8(3)$ & C11 & C15 & $\mathrm{C} 7$ & $113.1(3)$ \\
\hline C7 & C6 & C5 & $121.3(3)$ & C14 & C15 & C1 & $103.7(3)$ \\
\hline C6 & $\mathrm{C} 7$ & C8 & $107.7(3)$ & C14 & C15 & C7 & $112.6(3)$ \\
\hline C6 & $\mathrm{C} 7$ & C15 & 113.2(3) & C14 & C15 & C11 & $100.3(3)$ \\
\hline C8 & $\mathrm{C7}$ & C15 & $109.4(3)$ & & & & \\
\hline
\end{tabular}

Table 6: Torsion Angles for SI-3. 


\begin{tabular}{|c|c|c|c|c|c|c|c|c|c|}
\hline A & B & C & D & Angle $^{\circ}$ & A & B & C & D & Angle $^{\circ}$ \\
\hline $\mathrm{O} 1$ & C1 & $\mathrm{C} 2$ & C3 & $104.2(4)$ & C8 & $\mathrm{C} 7$ & C15 & C1 & $168.8(3)$ \\
\hline $\mathrm{O} 1$ & C1 & $\mathrm{C} 2$ & C16 & $-18.0(5)$ & C8 & $\mathrm{C7}$ & C15 & C11 & $-60.5(4)$ \\
\hline $\mathrm{O} 1$ & C1 & C15 & $\mathrm{C} 7$ & $-112.3(4)$ & $\mathrm{C} 8$ & $\mathrm{C} 7$ & C15 & C14 & $52.3(4)$ \\
\hline $\mathrm{O} 1$ & C1 & C15 & C11 & $117.5(4)$ & $\mathrm{C} 8$ & C9 & C10 & C11 & $-3.6(6)$ \\
\hline O1 & C1 & C15 & C14 & $9.4(4)$ & C9 & C10 & C11 & C12 & $-125.2(4)$ \\
\hline $\mathrm{O} 2$ & C14 & C15 & C1 & $-93.0(4)$ & $\mathrm{C9}$ & C10 & C11 & C15 & $-10.0(5)$ \\
\hline $\mathrm{O} 2$ & C14 & C15 & $\mathrm{C} 7$ & $28.4(5)$ & C10 & C11 & C12 & C13 & $84.5(4)$ \\
\hline $\mathrm{O} 2$ & C14 & C15 & C11 & $148.8(4)$ & C10 & C11 & C12 & C20 & $-151.7(3)$ \\
\hline C1 & $\mathrm{C} 2$ & C3 & $\mathrm{C} 4$ & $85.1(4)$ & C10 & C11 & C15 & C1 & $171.3(3)$ \\
\hline $\mathrm{C} 2$ & C1 & C15 & $\mathrm{C} 7$ & $65.2(4)$ & C10 & C11 & C15 & $\mathrm{C} 7$ & $41.6(4)$ \\
\hline $\mathrm{C} 2$ & C1 & C15 & C11 & $-65.0(4)$ & C10 & C11 & C15 & C14 & $-78.5(3)$ \\
\hline C2 & C1 & C15 & C14 & $-173.1(3)$ & C11 & C12 & C13 & C14 & $13.7(4)$ \\
\hline $\mathrm{C} 2$ & C3 & C4 & C5 & $-136.9(4)$ & C12 & C11 & C15 & $\mathrm{C} 1$ & $-71.4(4)$ \\
\hline $\mathrm{C} 2$ & C3 & C4 & C6 & $-63.2(5)$ & C12 & C11 & C15 & $\mathrm{C} 7$ & $158.9(3)$ \\
\hline C3 & $\mathrm{C} 4$ & C5 & C6 & $108.5(4)$ & C12 & C11 & C15 & C14 & $38.8(3)$ \\
\hline C3 & C4 & C5 & C17 & $-1.0(6)$ & C12 & C13 & C14 & $\mathrm{O} 2$ & $-168.6(4)$ \\
\hline C3 & $\mathrm{C} 4$ & C5 & C18 & $-144.1(4)$ & C12 & C13 & C14 & C15 & $10.7(4)$ \\
\hline C3 & $\mathrm{C} 4$ & C6 & C5 & $-116.0(4)$ & C13 & C14 & C15 & $\mathrm{C} 1$ & $87.6(3)$ \\
\hline C3 & $\mathrm{C} 4$ & C6 & $\mathrm{C} 7$ & $-6.1(6)$ & C13 & C14 & C15 & $\mathrm{C} 7$ & $-150.9(3)$ \\
\hline C4 & C5 & C6 & $\mathrm{C} 7$ & $-112.2(4)$ & C13 & C14 & C15 & C11 & $-30.5(4)$ \\
\hline C4 & C6 & $\mathrm{C7}$ & C8 & $-173.7(3)$ & C15 & $\mathrm{C} 1$ & $\mathrm{C} 2$ & C3 & $-73.3(4)$ \\
\hline C4 & C6 & $\mathrm{C} 7$ & C15 & $65.2(5)$ & C15 & C1 & $\mathrm{C} 2$ & C16 & $164.5(3)$ \\
\hline C5 & $\mathrm{C} 4$ & C6 & $\mathrm{C} 7$ & $109.9(4)$ & C15 & $\mathrm{C} 7$ & $\mathrm{C} 8$ & $\mathrm{C} 9$ & $45.0(4)$ \\
\hline C5 & C6 & $\mathrm{C} 7$ & $\mathrm{C} 8$ & $-101.8(4)$ & C15 & C11 & C12 & C13 & $-33.4(4)$ \\
\hline $\mathrm{C} 5$ & $\mathrm{C} 6$ & $\mathrm{C} 7$ & C15 & $137.1(3)$ & $\mathrm{C} 15$ & $\mathrm{C} 11$ & $\mathrm{C} 12$ & $\mathrm{C} 20$ & $90.5(4)$ \\
\hline
\end{tabular}




\begin{tabular}{llllllllll}
\hline $\mathbf{A}$ & $\mathbf{B}$ & $\mathbf{C}$ & $\mathbf{D}$ & Angle $^{\circ}$ & $\mathbf{A}$ & $\mathbf{B}$ & $\mathbf{C}$ & $\mathbf{D}$ & Angle $^{\circ}$ \\
$\mathrm{C} 6$ & $\mathrm{C} 4$ & $\mathrm{C} 5$ & $\mathrm{C} 17$ & $-109.5(4)$ & $\mathrm{C} 16$ & $\mathrm{C} 2$ & $\mathrm{C} 3$ & $\mathrm{C} 4$ & $-153.3(3)$ \\
$\mathrm{C} 6$ & $\mathrm{C} 4$ & $\mathrm{C} 5$ & $\mathrm{C} 18$ & $107.4(4)$ & $\mathrm{C} 17$ & $\mathrm{C} 5$ & $\mathrm{C} 6$ & $\mathrm{C} 4$ & $110.7(4)$ \\
$\mathrm{C} 6$ & $\mathrm{C} 7$ & $\mathrm{C} 8$ & $\mathrm{C} 9$ & $-78.4(4)$ & $\mathrm{C} 17$ & $\mathrm{C} 5$ & $\mathrm{C} 6$ & $\mathrm{C} 7$ & $-1.5(5)$ \\
$\mathrm{C} 6$ & $\mathrm{C} 7$ & $\mathrm{C} 15$ & $\mathrm{C} 1$ & $-71.0(4)$ & $\mathrm{C} 18$ & $\mathrm{C} 5$ & $\mathrm{C} 6$ & $\mathrm{C} 4$ & $-106.1(4)$ \\
$\mathrm{C} 6$ & $\mathrm{C} 7$ & $\mathrm{C} 15$ & $\mathrm{C} 11$ & $59.7(4)$ & $\mathrm{C} 18$ & $\mathrm{C} 5$ & $\mathrm{C} 6$ & $\mathrm{C} 7$ & $141.7(4)$ \\
$\mathrm{C} 6$ & $\mathrm{C} 7$ & $\mathrm{C} 15$ & $\mathrm{C} 14$ & $172.4(3)$ & $\mathrm{C} 19$ & $\mathrm{C} 9$ & $\mathrm{C} 10$ & $\mathrm{C} 11$ & $176.6(4)$ \\
$\mathrm{C} 7$ & $\mathrm{C} 8$ & $\mathrm{C} 9$ & $\mathrm{C} 10$ & $-15.1(5)$ & $\mathrm{C} 20$ & $\mathrm{C} 12$ & $\mathrm{C} 13$ & $\mathrm{C} 14$ & $-109.3(4)$ \\
$\mathrm{C} 7$ & $\mathrm{C} 8$ & $\mathrm{C} 9$ & $\mathrm{C} 19$ & $164.7(3)$ & & & & & \\
\hline
\end{tabular}

Table 7: Hydrogen Atom Coordinates $\left(\AA \times 10^{4}\right)$ and Isotropic Displacement Parameters $\left(\AA^{2} \times 10^{3}\right)$ for SI-3.

\begin{tabular}{ccccc}
\hline Atom & $\boldsymbol{x}$ & $\boldsymbol{y}$ & $\boldsymbol{z}$ & U(eq) \\
H2 & 1771.35 & 4173.2 & 2050.94 & 38 \\
H3A & -1533.21 & 4855.49 & 660.79 & 40 \\
H3B & -1747.61 & 5009.74 & 2614.85 & 40 \\
H4 & 1510.07 & 5562.18 & 947.38 & 39 \\
H6 & 3505.65 & 5926.43 & 3585.54 & 38 \\
H7 & 344.83 & 5522.72 & 5553.41 & 37 \\
H8A & 3092.83 & 6436.52 & 6593.38 & 39 \\
H8B & 2729.8 & 5824.09 & 7966.32 & 39 \\
H10 & 7499.75 & 4949.81 & 6539.54 & 41 \\
H11 & 4784.4 & 4428.4 & 4171.4 & 37 \\
H12 & 6671.38 & 3651.48 & 6804.63 & 40 \\
H13A & 3191.69 & 3175.36 & 7820.92 & 42 \\
H13B & 4835.7 & 3721.61 & 8923.92 & 42 \\
H16A & -125.86 & 3021.59 & 2043.68 & \\
\hline
\end{tabular}




\begin{tabular}{ccccc}
\hline Atom & $\boldsymbol{x}$ & $\boldsymbol{y}$ & $\boldsymbol{z}$ & U(eq) \\
H16B & -877.38 & 3489.42 & 328.47 & 60 \\
H16C & -2200.35 & 3487.92 & 1827.27 & 60 \\
H17A & -2573.87 & 6700.69 & 2415.05 & 60 \\
H17B & -1204.96 & 6918.1 & 4224.8 & 60 \\
H17C & -2002.59 & 6087.21 & 3881.33 & 60 \\
H18A & 2516.37 & 6848.83 & 1351.65 & 70 \\
H18B & 1495.99 & 7413.89 & 2503.59 & 70 \\
H18C & 222.08 & 7127.35 & 711.54 & 70 \\
H19A & 6729.97 & 6251.78 & 9299.88 & 62 \\
H19B & 6917.24 & 6689.78 & 7577.83 & 62 \\
H19C & 8410.66 & 6010.12 & 8195.47 & 62 \\
H20A & 3248.49 & 3015.25 & 4470.98 & 56 \\
H20B & 4996.12 & 2533.73 & 5626.76 & 56 \\
H20C & 5539.44 & 3042.49 & 4117.05 & \\
\hline
\end{tabular}

X-ray Crystal Structure for 20

CCDC number 2047290 

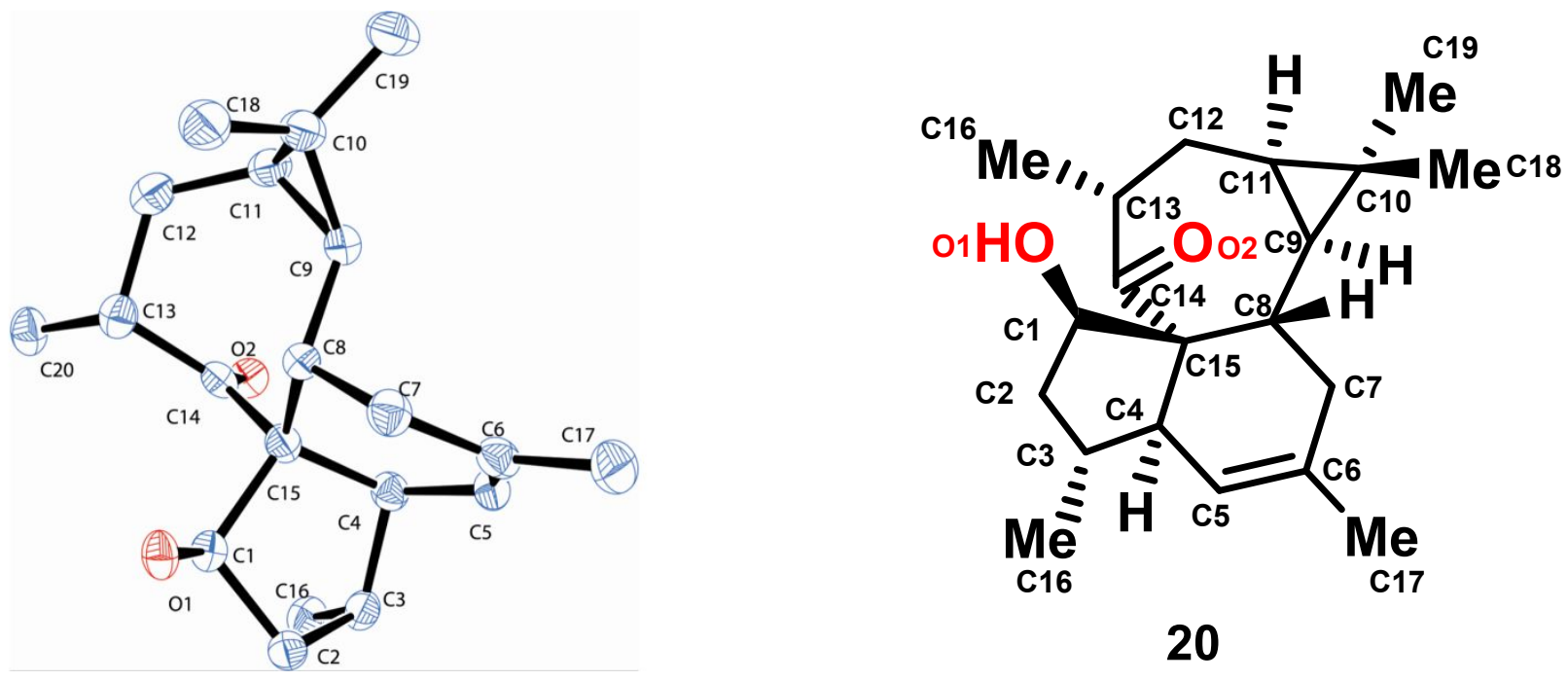

Figure 1: ORTEP representation of 20 (left) and 2D representation of 20 (right) with defined stereocenters.

Table 1: Crystal data and structure refinement for 20 (CCDC2047290).

$\begin{array}{ll}\text { Empirical formula } & \mathrm{C}_{20} \mathrm{H}_{30} \mathrm{O}_{2} \\ \text { Formula weight } & 302.44\end{array}$




\begin{tabular}{|c|c|c|}
\hline Temperature & $100(2) \mathrm{K}$ & \\
\hline Wavelength & $0.71073 \AA$ & \\
\hline Crystal system & Orthorhombic & \\
\hline Space group & P 212121 & \\
\hline \multirow[t]{3}{*}{ Unit cell dimensions } & $\mathrm{a}=9.404(2) \AA$ & $\alpha=90^{\circ}$. \\
\hline & $\mathrm{b}=10.494(3) \AA$ & $\beta=90^{\circ}$. \\
\hline & $\mathrm{c}=17.974(4) \AA$ & $\gamma=90^{\circ}$. \\
\hline Volume & $1773.7(7) \AA^{3}$ & \\
\hline $\mathrm{Z}$ & 4 & \\
\hline Density (calculated) & $1.133 \mathrm{Mg} / \mathrm{m}^{3}$ & \\
\hline Absorption coefficient & $0.071 \mathrm{~mm}^{-1}$ & \\
\hline $\mathrm{F}(000)$ & 664 & \\
\hline Crystal size & $0.200 \times 0.150 \times 0.050 \mathrm{~mm}^{3}$ & \\
\hline Theta range for data collection & 2.247 to $29.280^{\circ}$. & \\
\hline \multirow[t]{2}{*}{ Index ranges } & $-11<=\mathrm{h}<=12,-13<=\mathrm{k}<=14,-$ & \\
\hline & $24<=1<=20$ & \\
\hline Reflections collected & 9308 & \\
\hline Independent reflections & $4809[\mathrm{R}(\mathrm{int})=0.0555]$ & \\
\hline Completeness to theta $=25.242^{\circ}$ & $99.9 \%$ & \\
\hline \multirow[t]{2}{*}{ Absorption correction } & Semi-empirical from & \\
\hline & equivalents & \\
\hline Max. and min. transmission & 0.9919 and 0.7225 & \\
\hline Refinement method & Full-matrix least-squares on $\mathrm{F}^{2}$ & \\
\hline Data / restraints / parameters & 4809 / 0 / 206 & \\
\hline Goodness-of-fit on $\mathrm{F}^{2}$ & 1.050 & \\
\hline Final $R$ indices $[\mathrm{I}>2 \operatorname{sigma}(\mathrm{I})]$ & $\mathrm{R} 1=0.0705, \mathrm{wR} 2=0.1438$ & \\
\hline $\mathrm{R}$ indices (all data) & $\mathrm{R} 1=0.1224, \mathrm{wR} 2=0.1914$ & \\
\hline Absolute structure parameter & $3.3(10)$ & \\
\hline Extinction coefficient & $0.011(2)$ & \\
\hline Largest diff. peak and hole & 0.311 and -0.373 e. $\AA^{-3}$ & \\
\hline
\end{tabular}


Table 2: Atomic coordinates $\left(x 10^{4}\right)$ and equivalent isotropic displacement parameters $\left(\AA^{2} \times 10^{3}\right)$ for 20. $\mathrm{U}(\mathrm{eq})$ is defined as one third of the trace of the orthogonalized $\mathrm{Uij}^{\mathrm{ij}}$ tensor.

\begin{tabular}{|c|c|c|c|c|}
\hline & $\mathrm{x}$ & $\mathrm{y}$ & $\mathrm{z}$ & $\mathrm{U}(\mathrm{eq})$ \\
\hline $\mathrm{O}(1)$ & 2793(3) & $5028(3)$ & $3090(2)$ & $24(1)$ \\
\hline $\mathrm{O}(2)$ & $5246(3)$ & 8711(3) & $2740(2)$ & $25(1)$ \\
\hline $\mathrm{C}(1)$ & $3537(5)$ & 6095(4) & $3371(2)$ & $23(1)$ \\
\hline$C(2)$ & $3171(5)$ & $6318(4)$ & $4186(2)$ & $24(1)$ \\
\hline$C(3)$ & $3472(5)$ & $7725(4)$ & 4323(3) & $24(1)$ \\
\hline$C(4)$ & $2854(5)$ & $8350(4)$ & $3614(2)$ & $23(1)$ \\
\hline$C(5)$ & $1294(5)$ & $8600(4)$ & $3723(3)$ & $26(1)$ \\
\hline$C(6)$ & $230(5)$ & $8026(5)$ & $3365(3)$ & $26(1)$ \\
\hline$C(7)$ & $523(5)$ & $7070(5)$ & 2767(3) & $27(1)$ \\
\hline$C(8)$ & 1970(4) & $7263(4)$ & 2391(2) & $22(1)$ \\
\hline $\mathrm{C}(9)$ & 1903(5) & 8391(4) & 1859(3) & $25(1)$ \\
\hline$C(10)$ & $1583(6)$ & $8237(5)$ & 1047(3) & $30(1)$ \\
\hline $\mathrm{C}(11)$ & $3047(5)$ & $8681(5)$ & $1284(2)$ & $28(1)$ \\
\hline$C(12)$ & $4403(5)$ & $7902(5)$ & $1180(3)$ & $30(1)$ \\
\hline$C(13)$ & $4882(5)$ & 7069(4) & 1843(3) & $25(1)$ \\
\hline$C(14)$ & $4508(5)$ & 7809(4) & 2543(2) & $22(1)$ \\
\hline$C(15)$ & $3180(4)$ & 7412(4) & $2970(2)$ & $22(1)$ \\
\hline$C(16)$ & $5058(5)$ & 7983(5) & 4445(3) & $29(1)$ \\
\hline $\mathrm{C}(17)$ & $-1306(5)$ & $8269(6)$ & $3552(3)$ & $36(1)$ \\
\hline$C(18)$ & $1440(6)$ & $6915(5)$ & $713(3)$ & $35(1)$ \\
\hline$C(19)$ & $610(6)$ & $9210(5)$ & 693(3) & $39(1)$ \\
\hline$C(20)$ & $6473(5)$ & 6781(4) & 1788(3) & $30(1)$ \\
\hline
\end{tabular}


Table 3: Bond lengths $[\AA]$ for 20.

\begin{tabular}{ll}
\hline $\mathrm{O}(1)-\mathrm{C}(1)$ & $1.414(5)$ \\
$\mathrm{O}(2)-\mathrm{C}(14)$ & $1.226(5)$ \\
$\mathrm{C}(1)-\mathrm{C}(2)$ & $1.524(6)$ \\
$\mathrm{C}(1)-\mathrm{C}(15)$ & $1.594(6)$ \\
$\mathrm{C}(2)-\mathrm{C}(3)$ & $1.524(6)$ \\
$\mathrm{C}(3)-\mathrm{C}(16)$ & $1.532(6)$ \\
$\mathrm{C}(3)-\mathrm{C}(4)$ & $1.546(6)$ \\
$\mathrm{C}(4)-\mathrm{C}(5)$ & $1.503(6)$ \\
$\mathrm{C}(4)-\mathrm{C}(15)$ & $1.550(6)$ \\
$\mathrm{C}(5)-\mathrm{C}(6)$ & $1.333(6)$ \\
$\mathrm{C}(6)-\mathrm{C}(7)$ & $1.496(7)$ \\
$\mathrm{C}(6)-\mathrm{C}(17)$ & $1.505(6)$ \\
$\mathrm{C}(7)-\mathrm{C}(8)$ & $1.533(6)$ \\
\hline
\end{tabular}

\begin{tabular}{ll}
\hline $\mathrm{C}(8)-\mathrm{C}(9)$ & $1.523(6)$ \\
$\mathrm{C}(8)-\mathrm{C}(15)$ & $1.550(6)$ \\
$\mathrm{C}(9)-\mathrm{C}(10)$ & $1.499(6)$ \\
$\mathrm{C}(9)-\mathrm{C}(11)$ & $1.523(6)$ \\
$\mathrm{C}(10)-\mathrm{C}(19)$ & $1.512(7)$ \\
$\mathrm{C}(10)-\mathrm{C}(11)$ & $1.515(7)$ \\
$\mathrm{C}(10)-\mathrm{C}(18)$ & $1.517(7)$ \\
$\mathrm{C}(11)-\mathrm{C}(12)$ & $1.526(7)$ \\
$\mathrm{C}(12)-\mathrm{C}(13)$ & $1.545(7)$ \\
$\mathrm{C}(13)-\mathrm{C}(14)$ & $1.519(6)$ \\
$\mathrm{C}(13)-\mathrm{C}(20)$ & $1.529(6)$ \\
$\mathrm{C}(14)-\mathrm{C}(15)$ & $1.523(6)$ \\
\hline
\end{tabular}


Table 4: Bond angles $\left[^{\circ}\right]$ for 20.

\begin{tabular}{lr}
\hline $\mathrm{O}(1)-\mathrm{C}(1)-\mathrm{C}(2)$ & $110.7(4)$ \\
$\mathrm{O}(1)-\mathrm{C}(1)-\mathrm{C}(15)$ & $114.9(4)$ \\
$\mathrm{C}(2)-\mathrm{C}(1)-\mathrm{C}(15)$ & $104.7(3)$ \\
$\mathrm{C}(1)-\mathrm{C}(2)-\mathrm{C}(3)$ & $105.2(4)$ \\
$\mathrm{C}(2)-\mathrm{C}(3)-\mathrm{C}(16)$ & $112.0(4)$ \\
$\mathrm{C}(2)-\mathrm{C}(3)-\mathrm{C}(4)$ & $102.0(4)$ \\
$\mathrm{C}(16)-\mathrm{C}(3)-\mathrm{C}(4)$ & $114.2(4)$ \\
$\mathrm{C}(5)-\mathrm{C}(4)-\mathrm{C}(3)$ & $109.5(4)$ \\
$\mathrm{C}(5)-\mathrm{C}(4)-\mathrm{C}(15)$ & $113.7(4)$ \\
$\mathrm{C}(3)-\mathrm{C}(4)-\mathrm{C}(15)$ & $105.8(3)$ \\
$\mathrm{C}(6)-\mathrm{C}(5)-\mathrm{C}(4)$ & $126.2(4)$ \\
$\mathrm{C}(5)-\mathrm{C}(6)-\mathrm{C}(7)$ & $120.8(4)$ \\
$\mathrm{C}(5)-\mathrm{C}(6)-\mathrm{C}(17)$ & $122.4(5)$ \\
$\mathrm{C}(7)-\mathrm{C}(6)-\mathrm{C}(17)$ & $116.8(4)$ \\
$\mathrm{C}(6)-\mathrm{C}(7)-\mathrm{C}(8)$ & $113.1(4)$ \\
$\mathrm{C}(9)-\mathrm{C}(8)-\mathrm{C}(7)$ & $110.1(4)$ \\
$\mathrm{C}(9)-\mathrm{C}(8)-\mathrm{C}(15)$ & $111.9(4)$ \\
$\mathrm{C}(7)-\mathrm{C}(8)-\mathrm{C}(15)$ & $111.6(4)$ \\
$\mathrm{C}(10)-\mathrm{C}(9)-\mathrm{C}(11)$ & $60.2(3)$ \\
$\mathrm{C}(10)-\mathrm{C}(9)-\mathrm{C}(8)$ & $122.3(4)$ \\
$\mathrm{C}(11)-\mathrm{C}(9)-\mathrm{C}(8)$ & $123.5(4)$ \\
$\mathrm{C}(9)-\mathrm{C}(10)-\mathrm{C}(19)$ & $117.3(4)$ \\
\hline & \\
\hline
\end{tabular}

\begin{tabular}{lr}
\hline $\mathrm{C}(9)-\mathrm{C}(10)-\mathrm{C}(11)$ & $60.7(3)$ \\
$\mathrm{C}(19)-\mathrm{C}(10)-\mathrm{C}(11)$ & $117.4(4)$ \\
$\mathrm{C}(9)-\mathrm{C}(10)-\mathrm{C}(18)$ & $120.1(4)$ \\
$\mathrm{C}(19)-\mathrm{C}(10)-\mathrm{C}(18)$ & $113.4(4)$ \\
$\mathrm{C}(11)-\mathrm{C}(10)-\mathrm{C}(18)$ & $118.3(4)$ \\
$\mathrm{C}(10)-\mathrm{C}(11)-\mathrm{C}(9)$ & $59.1(3)$ \\
$\mathrm{C}(10)-\mathrm{C}(11)-\mathrm{C}(12)$ & $124.1(4)$ \\
$\mathrm{C}(9)-\mathrm{C}(11)-\mathrm{C}(12)$ & $124.5(4)$ \\
$\mathrm{C}(11)-\mathrm{C}(12)-\mathrm{C}(13)$ & $116.9(4)$ \\
$\mathrm{C}(14)-\mathrm{C}(13)-\mathrm{C}(20)$ & $112.4(4)$ \\
$\mathrm{C}(14)-\mathrm{C}(13)-\mathrm{C}(12)$ & $106.4(4)$ \\
$\mathrm{C}(20)-\mathrm{C}(13)-\mathrm{C}(12)$ & $110.3(4)$ \\
$\mathrm{O}(2)-\mathrm{C}(14)-\mathrm{C}(13)$ & $120.1(4)$ \\
$\mathrm{O}(2)-\mathrm{C}(14)-\mathrm{C}(15)$ & $122.0(4)$ \\
$\mathrm{C}(13)-\mathrm{C}(14)-\mathrm{C}(15)$ & $117.9(4)$ \\
$\mathrm{C}(14)-\mathrm{C}(15)-\mathrm{C}(4)$ & $111.4(4)$ \\
$\mathrm{C}(14)-\mathrm{C}(15)-\mathrm{C}(8)$ & $106.9(3)$ \\
$\mathrm{C}(4)-\mathrm{C}(15)-\mathrm{C}(8)$ & $114.8(4)$ \\
$\mathrm{C}(14)-\mathrm{C}(15)-\mathrm{C}(1)$ & $107.0(3)$ \\
$\mathrm{C}(4)-\mathrm{C}(15)-\mathrm{C}(1)$ & $104.8(3)$ \\
$\mathrm{C}(8)-\mathrm{C}(15)-\mathrm{C}(1)$ & $111.7(3)$ \\
\hline
\end{tabular}


Table 5: Anisotropic displacement parameters $\left(\AA^{2} \times 10^{3}\right)$ for 20. The anisotropic displacement factor exponent takes the form: $-2 \pi^{2}\left[h^{2} a^{* 2} U^{11}+\ldots+2 h k a^{*} b^{*} U^{12}\right]$

\begin{tabular}{lllllll}
\hline & $\mathrm{U}^{11}$ & $\mathrm{U}^{22}$ & $\mathrm{U}^{33}$ & $\mathrm{U}^{23}$ & $\mathrm{U}^{13}$ & $\mathrm{U}^{12}$ \\
\hline $\mathrm{O}(1)$ & $24(2)$ & $17(1)$ & $32(2)$ & $-5(1)$ & $2(1)$ & $0(1)$ \\
$\mathrm{O}(2)$ & $24(2)$ & $24(2)$ & $27(2)$ & $-1(1)$ & $0(1)$ & $-5(1)$ \\
$\mathrm{C}(1)$ & $23(2)$ & $22(2)$ & $23(2)$ & $-2(2)$ & $1(2)$ & $1(2)$ \\
$\mathrm{C}(2)$ & $24(2)$ & $23(2)$ & $25(2)$ & $2(2)$ & $3(2)$ & $1(2)$ \\
$\mathrm{C}(3)$ & $25(2)$ & $23(2)$ & $24(2)$ & $-2(2)$ & $5(2)$ & $0(2)$ \\
$\mathrm{C}(4)$ & $25(2)$ & $20(2)$ & $24(2)$ & $-1(2)$ & $-1(2)$ & $1(2)$ \\
$\mathrm{C}(5)$ & $27(2)$ & $23(2)$ & $27(2)$ & $1(2)$ & $3(2)$ & $5(2)$ \\
$\mathrm{C}(6)$ & $21(2)$ & $27(2)$ & $28(2)$ & $5(2)$ & $-1(2)$ & $1(2)$ \\
$\mathrm{C}(7)$ & $22(2)$ & $30(2)$ & $30(2)$ & $1(2)$ & $0(2)$ & $1(2)$ \\
$\mathrm{C}(8)$ & $22(2)$ & $23(2)$ & $20(2)$ & $1(2)$ & $2(2)$ & $3(2)$ \\
$\mathrm{C}(9)$ & $28(2)$ & $24(2)$ & $23(2)$ & $-1(2)$ & $1(2)$ & $6(2)$ \\
$\mathrm{C}(10)$ & $39(3)$ & $25(2)$ & $25(2)$ & $1(2)$ & $-4(2)$ & $3(2)$ \\
$\mathrm{C}(11)$ & $37(3)$ & $25(2)$ & $21(2)$ & $1(2)$ & $1(2)$ & $-1(2)$ \\
$\mathrm{C}(12)$ & $34(2)$ & $32(3)$ & $25(2)$ & $-1(2)$ & $4(2)$ & $-3(2)$ \\
$\mathrm{C}(13)$ & $26(2)$ & $22(2)$ & $29(2)$ & $-4(2)$ & $2(2)$ & $-3(2)$ \\
$\mathrm{C}(14)$ & $22(2)$ & $19(2)$ & $26(2)$ & $4(2)$ & $-2(2)$ & $2(2)$ \\
$\mathrm{C}(15)$ & $21(2)$ & $20(2)$ & $25(2)$ & $2(2)$ & $1(2)$ & $1(2)$ \\
$\mathrm{C}(16)$ & $26(2)$ & $32(2)$ & $28(2)$ & $-2(2)$ & $1(2)$ & $0(2)$ \\
$\mathrm{C}(17)$ & $25(2)$ & $47(3)$ & $37(3)$ & $1(2)$ & $-2(2)$ & $8(2)$ \\
$\mathrm{C}(18)$ & $42(3)$ & $33(3)$ & $31(3)$ & $-4(2)$ & $-7(2)$ & $2(2)$ \\
$\mathrm{C}(19)$ & $50(3)$ & $36(3)$ & $30(3)$ & $3(2)$ & $-8(3)$ & $9(2)$ \\
$\mathrm{C}(20)$ & $30(2)$ & $22(2)$ & $39(3)$ & $-2(2)$ & $7(2)$ & $1(2)$ \\
\hline & & & & & \\
& $23)$ & & & \\
\end{tabular}




\section{References}

[1] Synthesis of a-methyl ketone 11: (a) Jorgensen, L.; McKerrall, S. J.; Kuttruff, C. A.; Ungeheuer, F.; Felding, J.; Baran, P. S. 14-Step Synthesis of (+)-Ingenol from (+)-3Carene. Science 2013, 341, 878-882; (b) McKerrall, S. J.; Jorgensen, L.; Kuttruff, C. A.; Ungeheuer, F.; Felding, J.; Baran, P. S. Development of a Concise Synthesis of (+)-Ingenol. J. Am. Chem. Soc. 2014, 136, 5799-5810.

[2] Preparation of (iodomethyl)triphenylphosphonium iodide: Seyferth, D.; Heeren, J. K.; Singh, G.; Grim, S. O.; Hughes, W. B. Studies in phosphinemethylene chemistry: XIII. Routes to triphenylphosphine-halomethylenes and -dihalomethylenes. J. Organomet. Chem. 1966, 5, 267-274.

[3] Isolation of pepluanol A: Wan, L.-S.; Nian, Y.; Ye, C.-J.; Shao, L.-D.; Peng, X.-R.; Shao, L.-D.; Geng, C.-A.; Zuo, Z.-L. Li, X.-N.; Yang, J.; Zhou, M.; Qiu, M.-H. Three Minor Diterpenoids with Three Carbon Skeletons from Euphorbia peplus. Org. Lett. 2016, 18, 2166-2169.

[4] Racemic synthesis of pepluanol A: Xuan, J.; Liu, Z.; Zhu, A.; Rao, P.; Yu, L.; Ding, H. Diastereoselective Total Synthesis of the Euphorbia Diterpenoid Pepluanol A: A Reductive Annulation Approach. Angew. Chem. Int. Ed. 2017, 56, 8898-8901.

[5] Gaussian 16, Revision C.01, Frisch, M. J.; Trucks, G. W.; Schlegel, H. B.; Scuseria, G. E.; Robb, M. A.; Cheeseman, J. R.; Scalmani, G.; Barone, V.; Petersson, G. A.; Nakatsuji, H.; Li, X.; Caricato, M.; Marenich, A. V.; Bloino, J.; Janesko, B. G.; Gomperts, R.; Mennucci, B.; Hratchian, H. P.; Ortiz, J. V.; Izmaylov, A. F.; Sonnenberg, J. L.; Williams-Young, D.; Ding, F.; Lipparini, F.; Egidi, F.; Goings, J.; Peng, B.; Petrone, A.; Henderson, T.; Ranasinghe, D.; Zakrzewski, V. G.; Gao, J.; Rega, N.; Zheng, G.; Liang, W.; Hada, M.; Ehara, M.; Toyota, K.; Fukuda, R.; Hasegawa, J.; Ishida, M.; Nakajima, T.; Honda, Y.; Kitao, O.; Nakai, H.; Vreven, T.; Throssell, K.; Montgomery, J. A., Jr.; Peralta, J. E.; Ogliaro, F.; Bearpark, M. J.; Heyd, J. J.; Brothers, E. N.; Kudin, K. N.; Staroverov, V. N.; Keith, T. A.; Kobayashi, R.; Normand, J.; Raghavachari, K.; Rendell, A. P.; Burant, J. C.; Iyengar, S. S.; Tomasi, J.; Cossi, M.; Millam, J. M.; Klene, M.; Adamo, C.; Cammi, R.; Ochterski, J. W.; Martin, R. L.; Morokuma, K.; Farkas, O.; Foresman, J. B.; Fox, D. J. Gaussian, Inc., Wallingford CT, 2016.

[6] Cossi, M.; Rega, N.; Scalmani, G.; Barone, V. Energies, structures, and electronic properties of molecules in solution with the C-PCM solvation model. J. Comput. Chem. 2003, 24, 669681.

[7] Hariharan, P. C.; Pople J. A. The influence of polarization functions on molecular orbital hydrogenation energies. Theor. Chim. Acta 1973, 28, 213-222. 
[8] Becke, A. D. Density-functional thermochemistry. III. The role of exact exchange. J. Chem. Phys. 1993, 98, 5648-5652. 